\title{
A study of university students in Japan: Learning and application of academic English writing
}

\author{
By \\ James Graham McKinley
}

\begin{abstract}
A thesis
submitted to the Victoria University of Wellington in fulfilment of the requirements for the degree of Doctor of Philosophy
\end{abstract}

VICTORIA UNIVERSITY OF WELLINGTON

Te Whare Wananga o te Upoko o te Ika a Maui

2012 


\section{Acknowledgments}

The journey of completing this thesis has involved a number of people who have supported me at various stages of the project in different ways. I dedicate this thesis to the most important person in the world who has been with me from the beginning to the end-Dr. Heath Rose.

I need to thank all the participants who opened up and remained open and encouraging throughout the entire data collection process. Without them there would be no study, and without these particular participants, it would not have been the enlightening and enjoyable experience it was.

For supervision and guidance, I cannot express enough gratitude for my main supervisor, Associate Professor John Macalister. His understanding and patience with all the delays and ability to steer me away from the seemingly endless need to either maintain tired constructs or take the project in new directions allowed me to finally understand what it means to complete a doctoral thesis. I thank my co-supervisor, Associate Professor Derek Wallace, who provided sturdy support and significant insight, and who provided the fresh pair of eyes to make sense of the final draft. Also, I especially thank Learning Support Advisor Kirsten Reid for her incredible endurance assisting me with my words over the years. I must also thank the academics who helped me get the project started in the first place at the University of Sydney, Professor Brian Paltridge, Dr. Paula MacAndrew, and my qualitative research guru, Professor Cherry Russell.

Finally I must thank my family around the world. From my parents and sisters in the United States, to my family and friends in Japan, and to my Wellington family in New Zealand, especially Eleanor Lefever, Andrea and Phil O’Brien, John MacArthur and James $\mathrm{Hu}$, I am so grateful for all the support and all you have done for me. I could not have done this without you. 


\section{Abstract}

This study investigated Japanese first and second year undergraduate students learning English academic writing in their compulsory English composition courses in a Japanese university. The thesis takes a social constructivist approach to investigate the aspects of critical argument and writer identity in these students' classes and their writing.

The data for the study include classroom observations and teacher and student interviews, all conducted monthly throughout the academic year-long course. In total there were six courses, four teachers, and sixteen student participants. The observations were analyzed using an adapted version of Ivanič's (2004) Discourses of Writing framework, which focused on aspects of identity construction in the writing classroom. The linguistic data included a selection of one major piece of writing from each student, analyzed using an adapted Appraisal framework within Systemic Functional Linguistics (Martin, 1997; 2000). In order to maintain a focus on writer identity in the analysis, Clark and Ivanič's (1997) selves were identified through this analysis. In addition, the texts were analyzed for use of Casanave's (2002) writing game strategies, in order to further establish the students' approaches in writing their texts. The objective was not to generalize about how Japanese students learn to write academic English, but rather to provide, from a social constructivist, Western researcher's perspective, an analysis of what happened in these students' writing classes and how it affected their writing for those classes.

Teachers' general practices in the observed courses mainly focused on two aspects of writing: 1) as a communicative act (writing for a reader), and 2) as an exercise in critical thinking (developing a thesis). These two aspects emerged from the observation and interview data collection. The four teachers used very different approaches in designing their courses, and the students in the same classes responded in different ways, mostly depending on their ability to understand their teachers' intentions and to form appropriate academic identities in an attempt to meet their teachers' expectations. The analysis of the students' written texts revealed that students often did not meet the teachers' expectations of writing objectively and using a genre-appropriate voice as students often resorted to the same authorial voice to push their thesis. 
This investigation was designed to inform pedagogic practices for university teachers of academic English and curriculum designers in Japan to establish effective English writing courses. The rich description of classroom practices and resulting written texts and the focus on differences in cultural expectations between teachers and students provide significant contributions to this area of inquiry. The main pedagogical suggestions are standardizing course objectives and goals, assigning more reading as a part of writing, and teaching students how to write authoritatively. 
Table of Contents

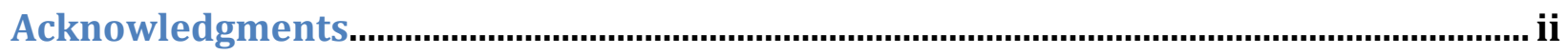

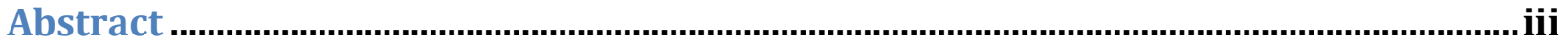

Chapter 1. Learning to write and reason: Introduction ....................................................... 7

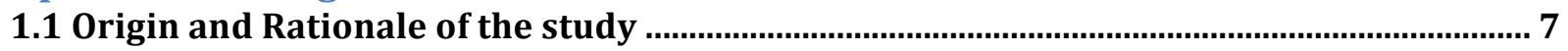

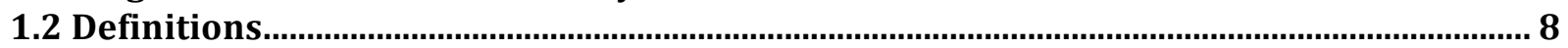

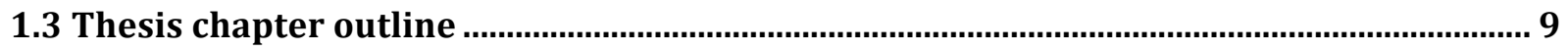

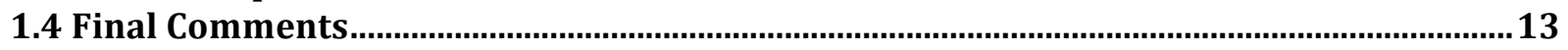

Chapter 2. Individualized voices and critical thinking: The literature review .............15

2.1 English L2 writing education: A brief overview ..................................................................

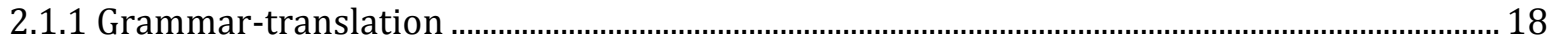

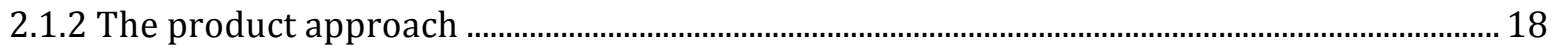

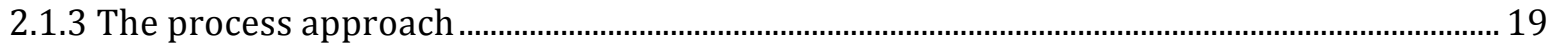

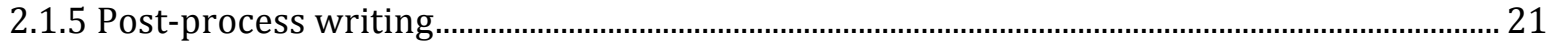

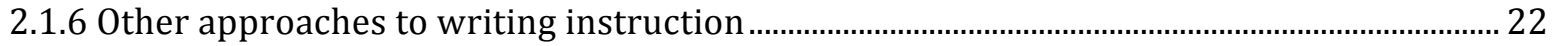

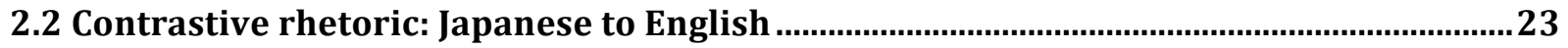

2.2.1 Structural differences between English and Japanese.............................................................. 24

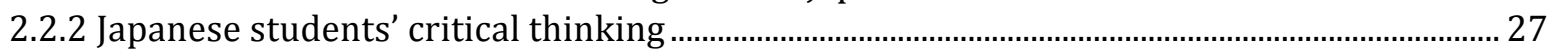

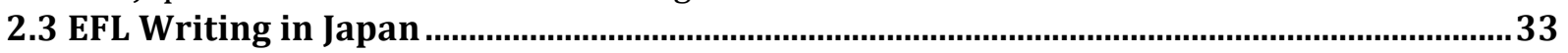

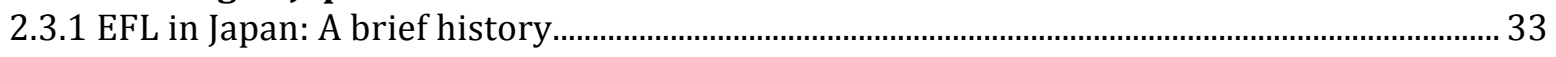

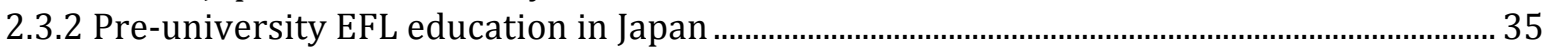

2.3.3 English L2 writing at the university level in Japan............................................................... 37

2.3.4 Application and debate of process writing in university English L2 writing in Japan........ 39

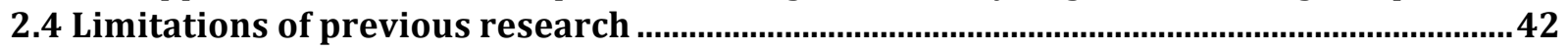

2.5 The growing concern for university-level writing education.............................................43

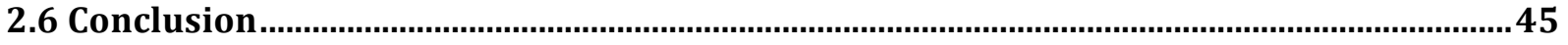

Chapter 3. Social constructivism: The theoretical framework ........................................47

3.1 Why focus on critical argument and writer identity? ......................................................47

3.2 Philosophy behind the study: Why social constructivism? ..................................................49

3.2.1 Social constructivism in education and identity construction .................................................. 49

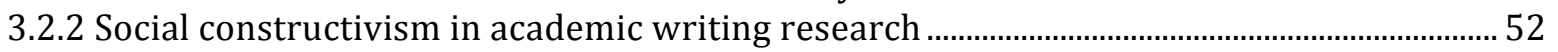

3.2.3 An overview of social constructivist research on L2 writing ............................................... 54

3.2.4 Social constructivist approaches in current L2 writing research .............................................5 57

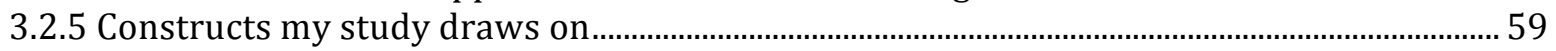

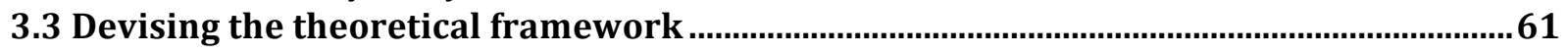

3.4 The phenomenon of constructing cultural and academic writer identities......................63

3.5 The phenomenon of developing critical argument in L2 writing ....................................68

3.6 Focus on critical thinking: Critical writing as mediated action ..........................................71

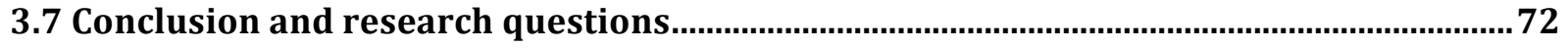

Chapter 4. Plans and paradigms: The methodological framework of the study..........75

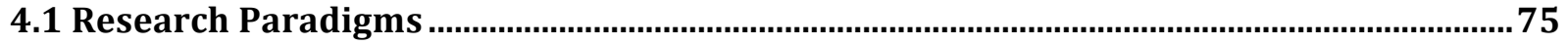

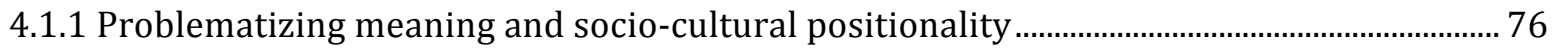

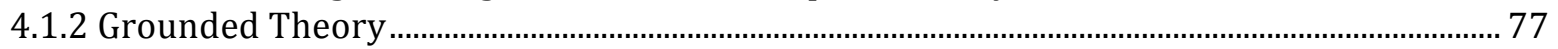

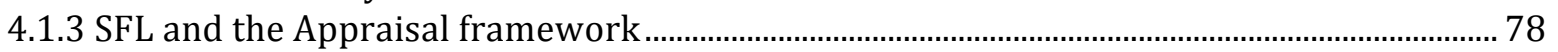

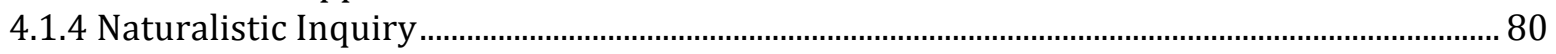

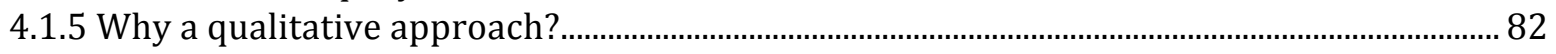


4.2 Research procedure

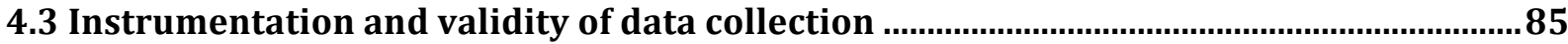

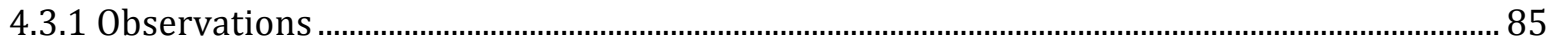

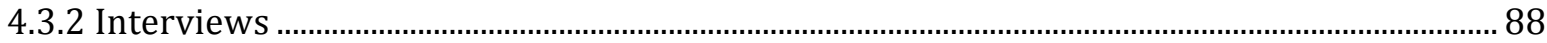

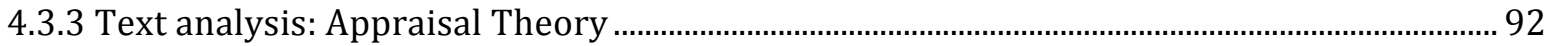

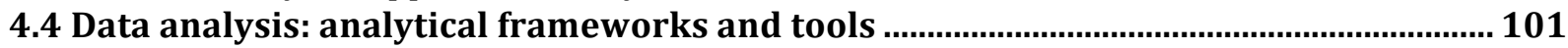

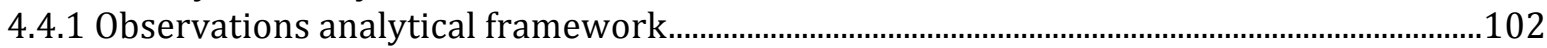

4.4.2 Text analysis analytical framework ……….............................................................................. 104

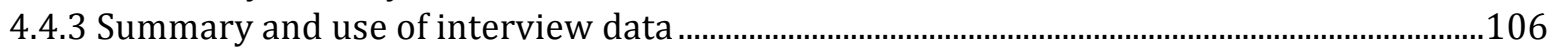

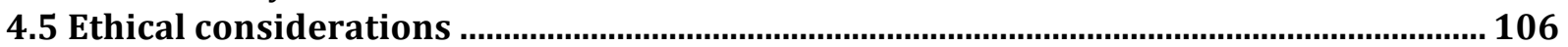

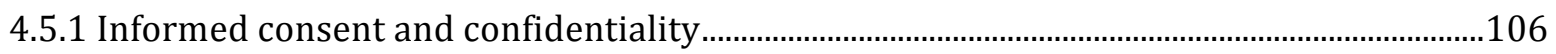

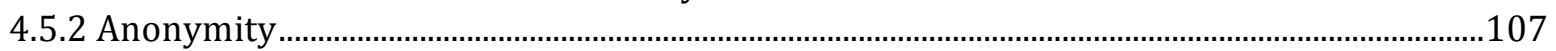

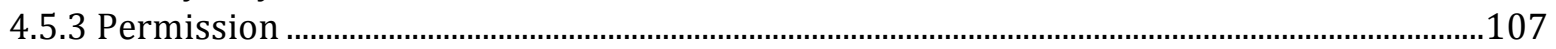

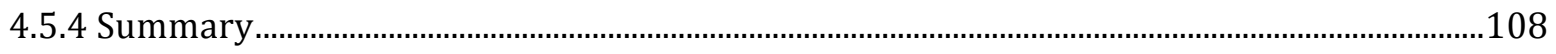

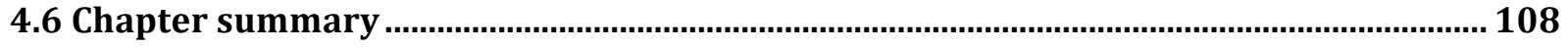

Chapter 5. Who, What, Where: Setting and participants ............................................... 109

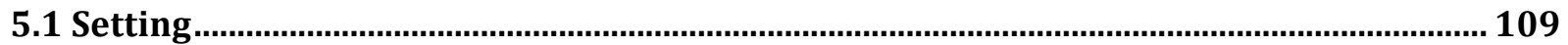

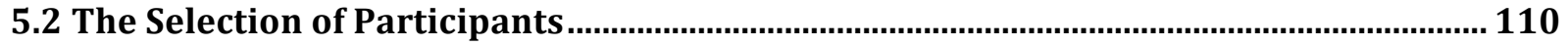

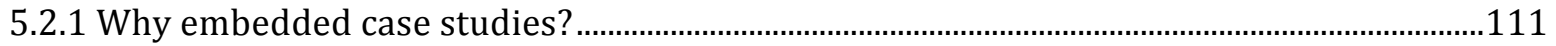

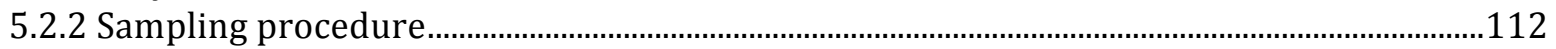

5.3 Description of participants

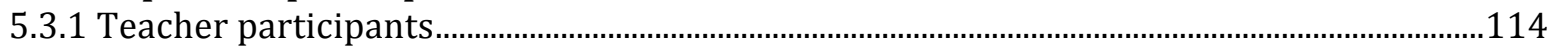

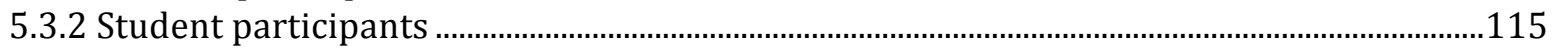

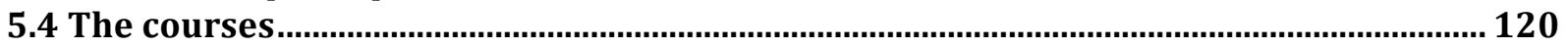

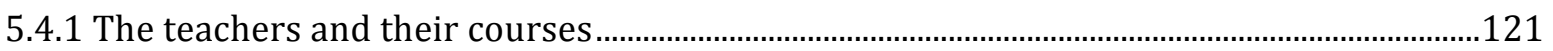

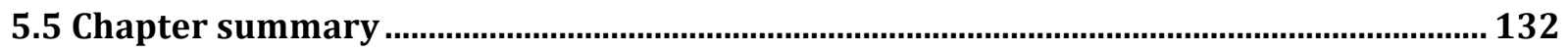

Chapter 6. Writing to be read: Observations, the discourses and readers................. 133

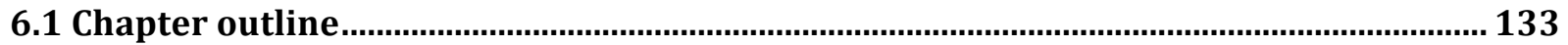

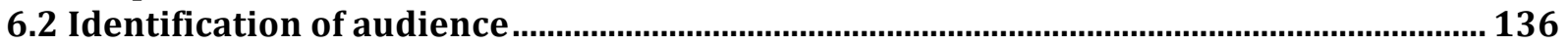

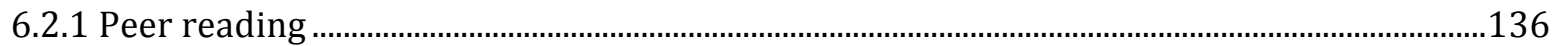

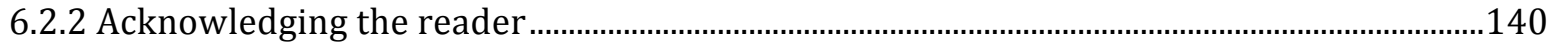

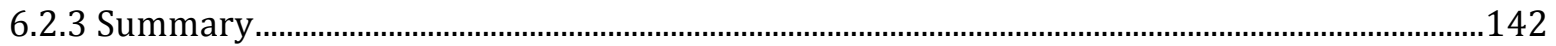

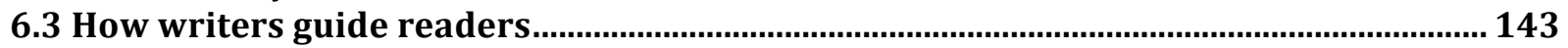

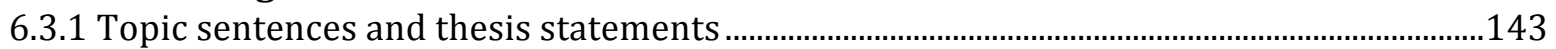

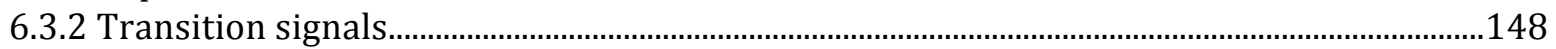

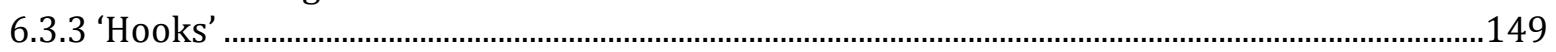

6.3.4 Questions to the reader

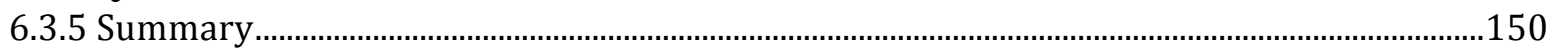

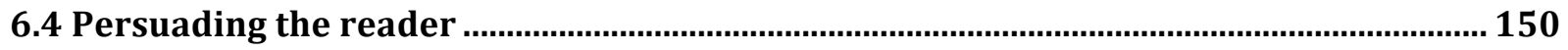

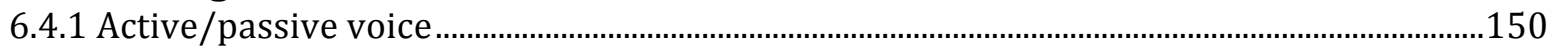

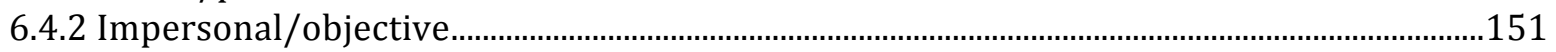

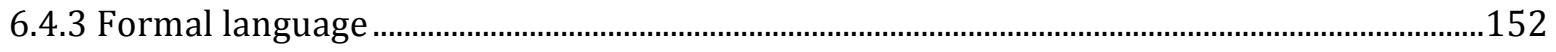

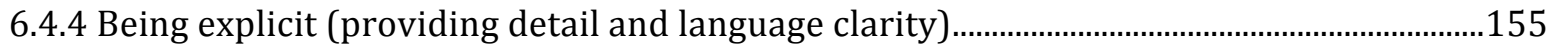

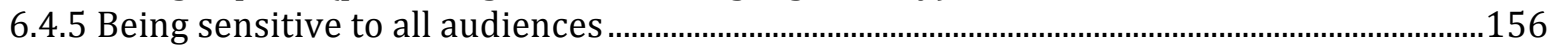

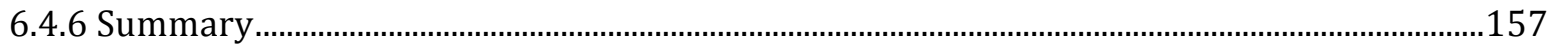

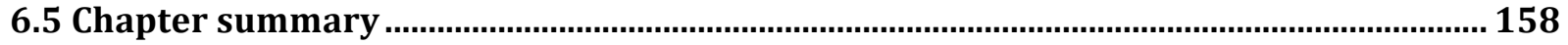

Chapter 7. More observations: Developing the thesis .....................................................159

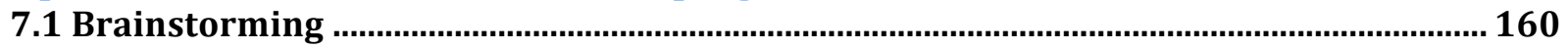




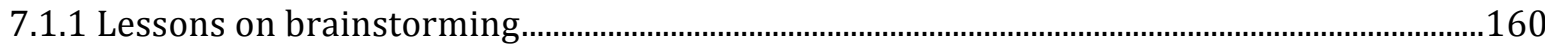

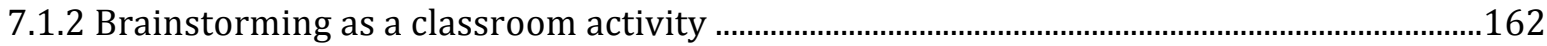

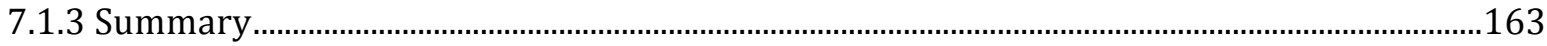

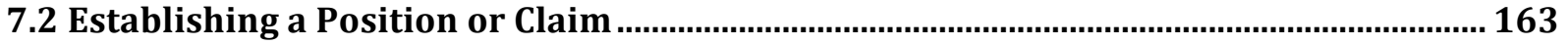

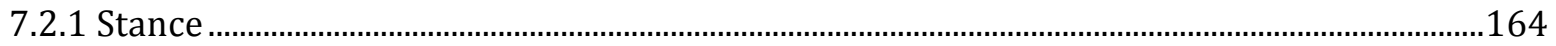

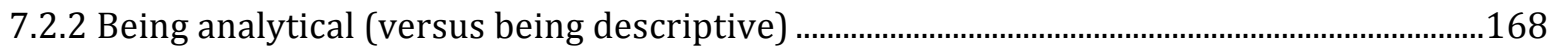

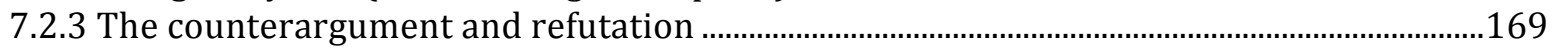

7.2.4 Establishing a position through discussion of feedback ……................................................171

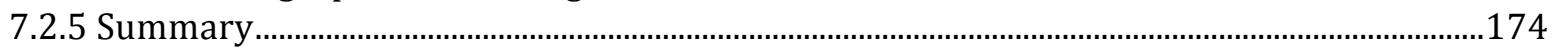

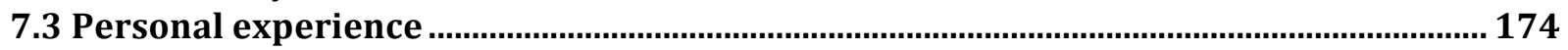

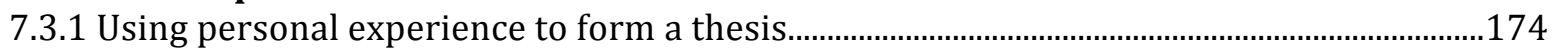

7.3.2 Using personal experience as supporting evidence.................................................................176

7.3.3 Discouraging use of personal evidence to make writing more objective...............................178

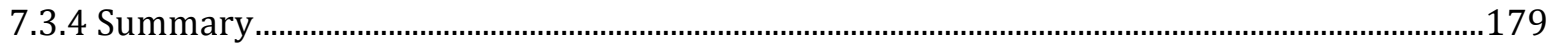

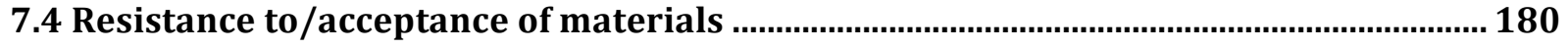

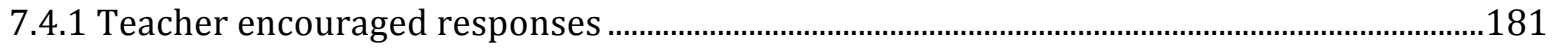

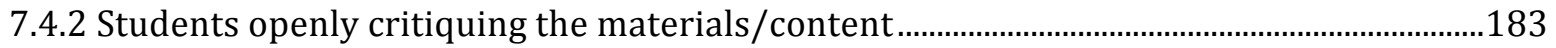

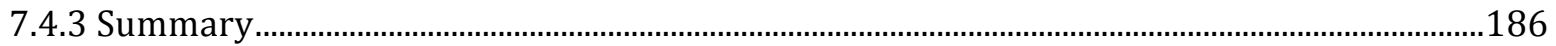

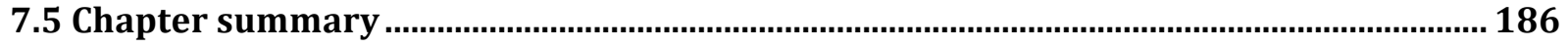

Chapter 8. Writing with multiple selves: Analysis of students' written texts............ 189

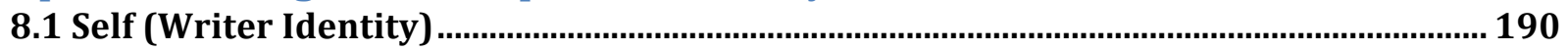

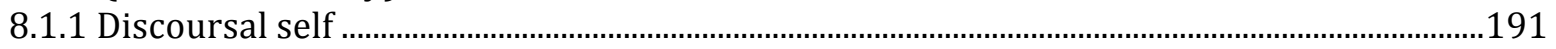

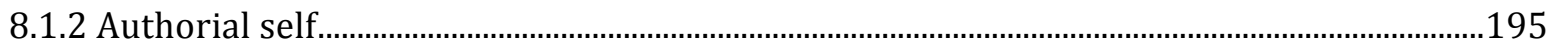

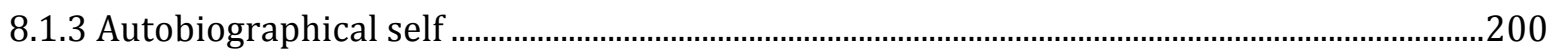

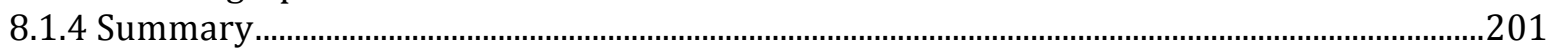

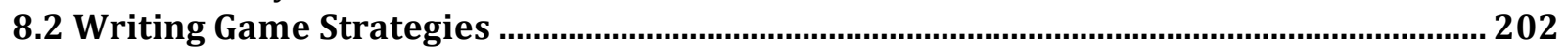

8.2.1 Game strategy 1: Interacting with texts and with others about texts ....................................203

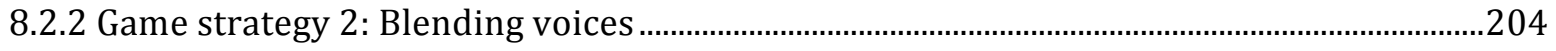

8.2.3 Game strategy 3: Owning research experiences and telling a good story from them ......207

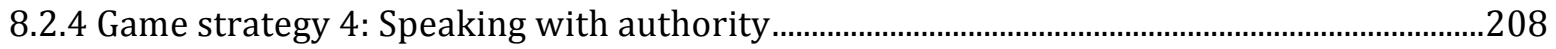

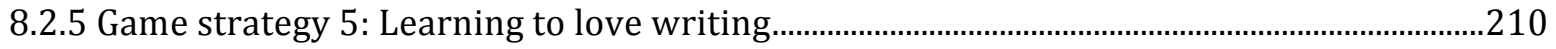

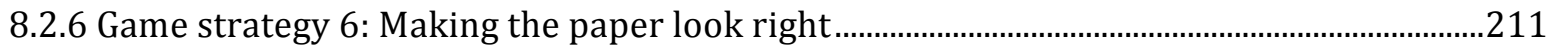

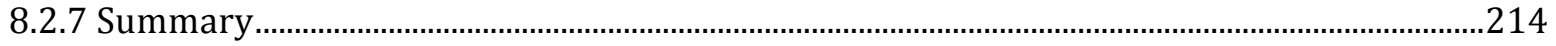

8.3 Case studies

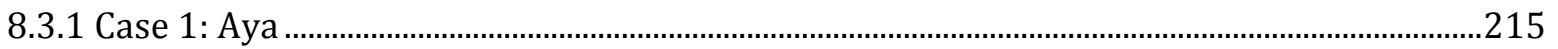

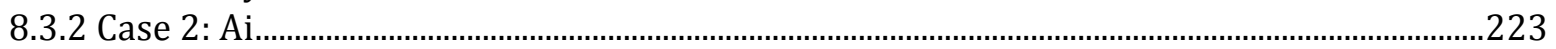

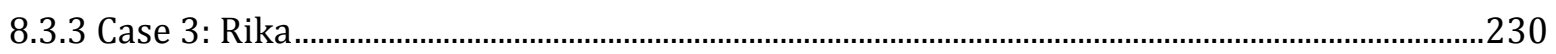

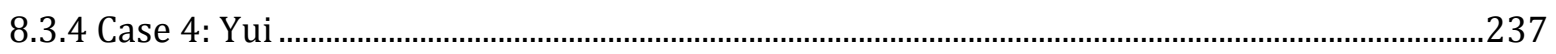

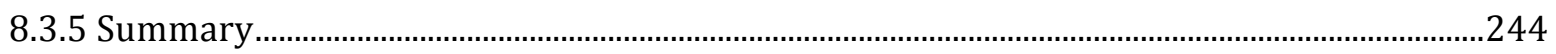

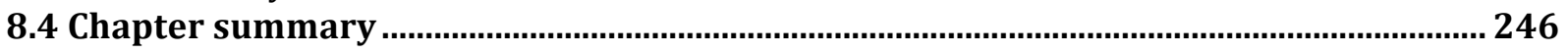

Chapter 9. Writing and reasoning revisited: Responding to the research questions

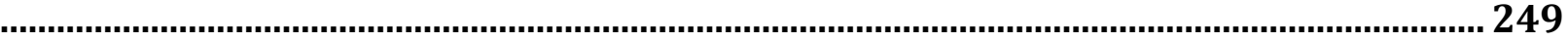

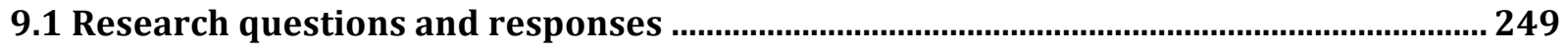

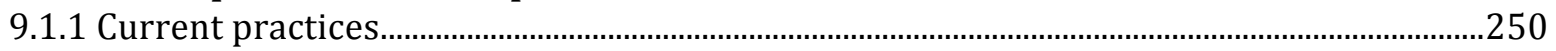

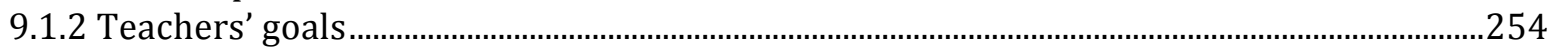

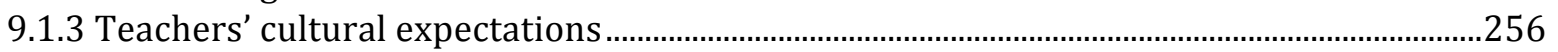

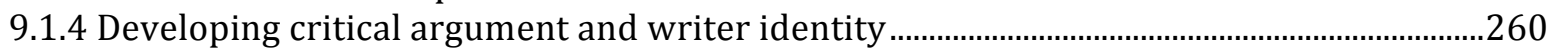

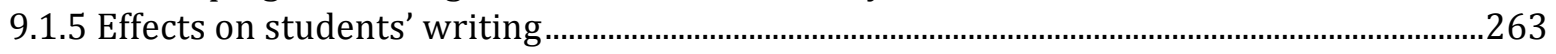

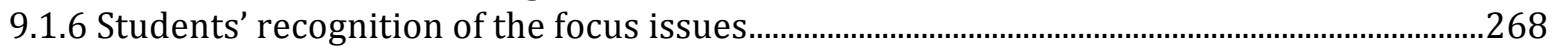




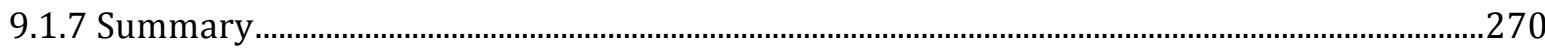

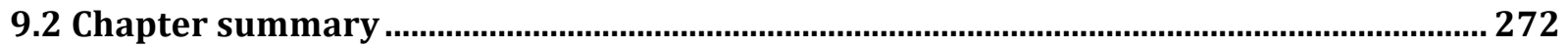

Chapter 10. So what? Conclusion and possibilities for the future ..................................2 275

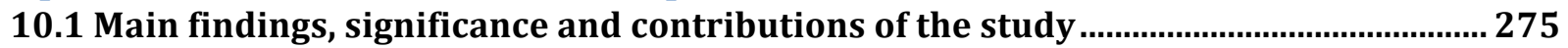

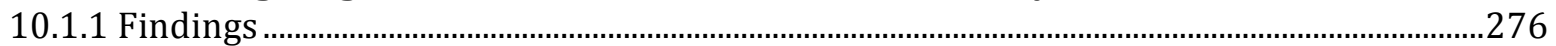

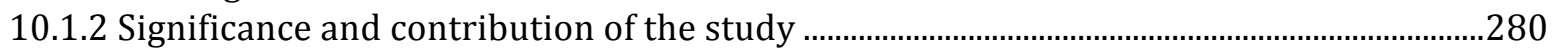

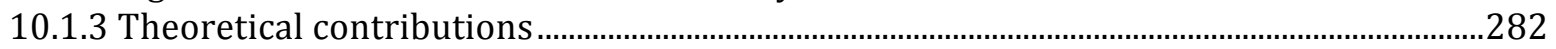

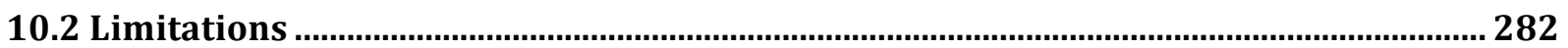

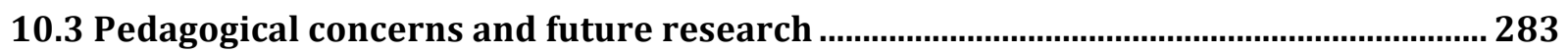

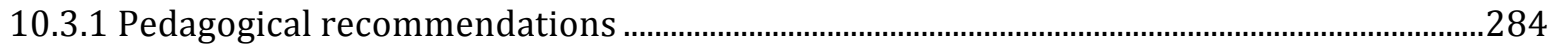

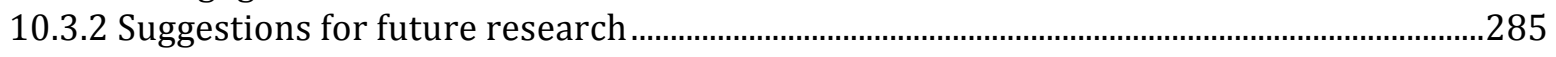

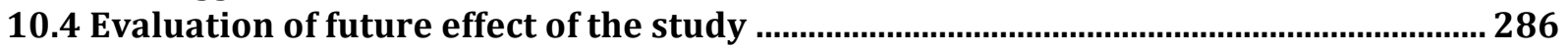

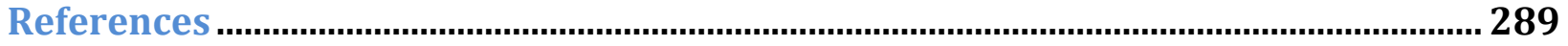

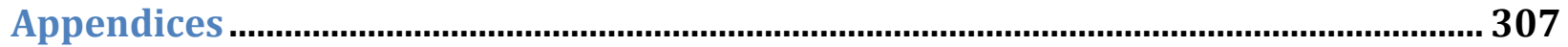

Appendix A

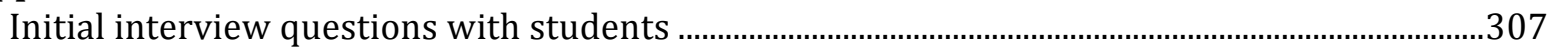

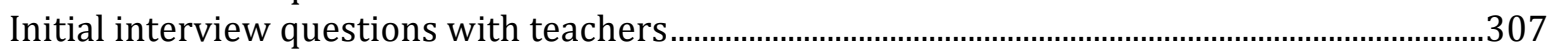

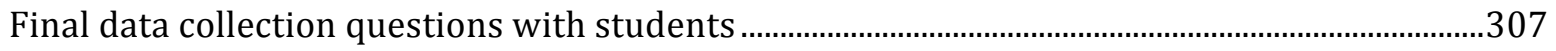

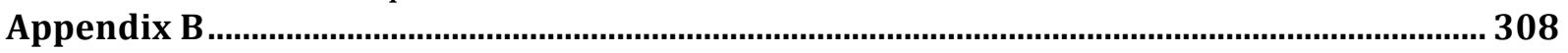

Participant information letter, statements and consent form ........................................................308

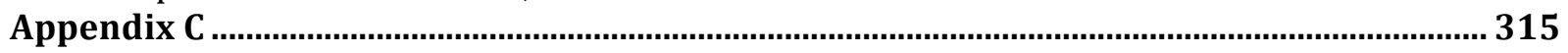

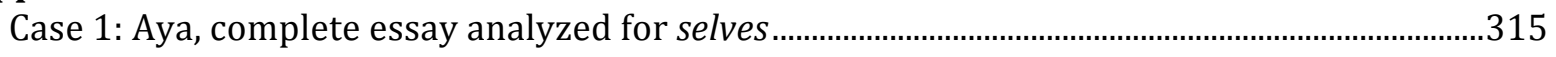

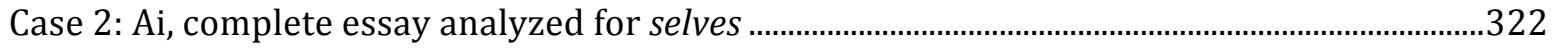

Case 3: Rika, complete essay analyzed for selves …….......................................................................... 327

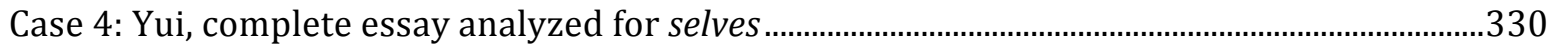

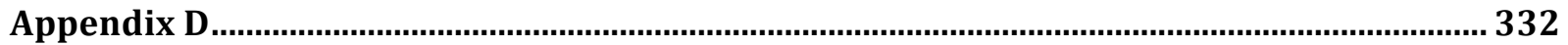

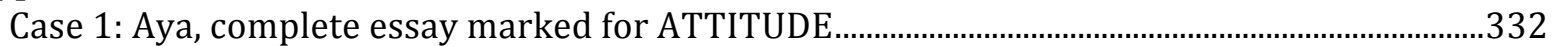

Case 1: Aya, complete essay marked for ENGAGEMENT ….................................................................339

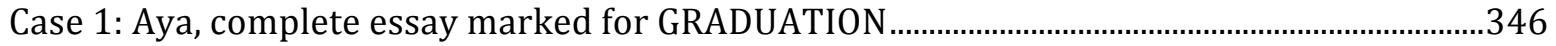

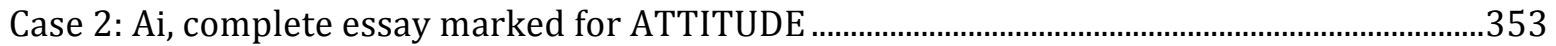

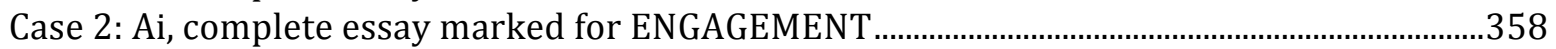

Case 2: Ai, complete essay marked for GRADUATION

Case 3: Rika, complete essay marked for ATTITUDE

Case 3: Rika, complete essay marked for ENGAGEMENT .................................................................371

Case 3: Rika, complete essay marked for GRADUATION ...................................................................374

Case 4: Yui, complete essay marked for ATTITUDE.........................................................................37

Case 4: Yui, complete essay marked for ENGAGEMENT ……...............................................................378

Case 4: Yui, complete essay marked for GRADUATION ..........................................................................379 


\section{LIST OF FIGURES AND TABLES}

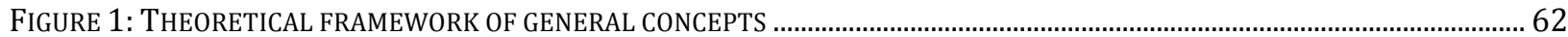

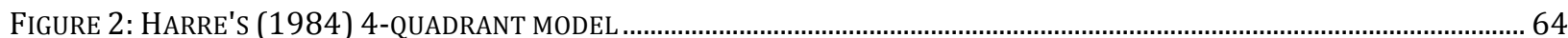

FIGURE 3: SUBJECT-POSITIONS; SOCIALLY AVAILABLE POSSIBILITIES FOR SELF-HOOD (CLARK \& IVANIČ, 1997, P.137) ........... 66

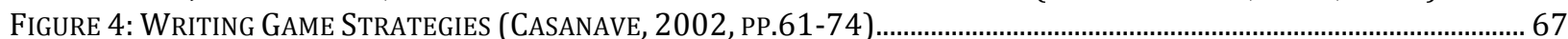

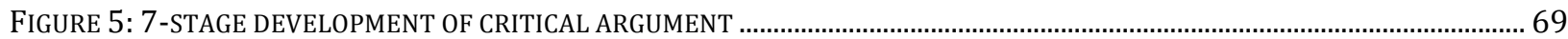

FIGURE 6: OUTLINE OF APPRAISAL RESOURCES IN ENGLISH (AS DESCRIBED BY MARTIN, 1997) .............................................. 95

FIGURE 7: EXPANDED OUTLINE FOR APPRAISAL FRAMEWORK FOR THIS STUDY, SHOWING INDICATORS AND SELVES .................. 98

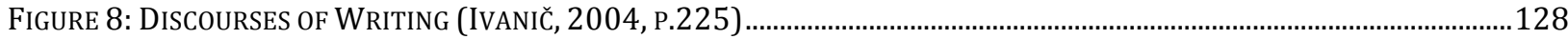

FIGURE 9: RELATIONSHIPS BETWEEN SIGNIFICANT FEATURES OF TEACHERS AND THEIR COURSES.........................................129

FIGURE 10: FUNCTIONS AND COUNTS OF TOPIC SENTENCE OR THESIS STATEMENT DISCUSSIONS.............................................144

TABLE 1: SUMMARY OF DATA COLLECTION INSTRUMENTS AND ANALYSIS, ADAPTED FROM MILES \& HUBERMAN (1994) ......... 84

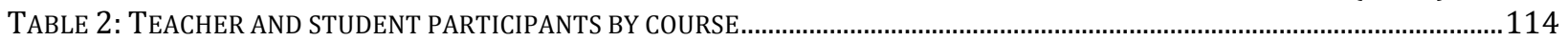

TABLE 3: KEY CHARACTERISTICS OF TEACHER PARTICIPANTS …............................................................................................ 115

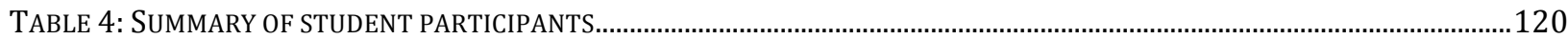

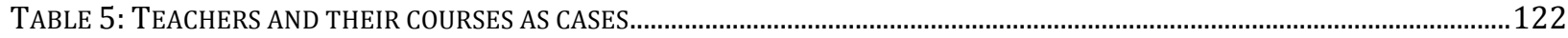

TABLE 6: THE DISCOURSES OF WRITING APPLIED BY EACH TEACHER

TABLE 7: SUB-INDICATORS AND FURTHER DIVISION OF THE WRITER'S UNDERSTANDING OF AUDIENCE ....................................135

TABLE 8: SUB-INDICATORS AND FURTHER DIVISION OF THE WRITER'S DEVELOPMENT OF A THESIS...........................................159

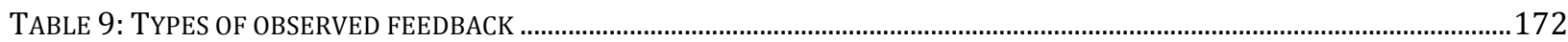

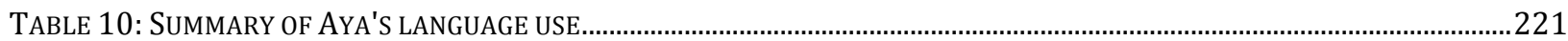

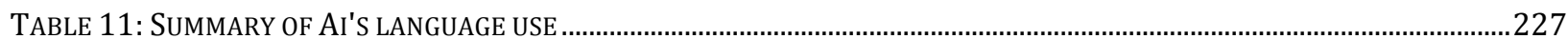

TABLE 12: SUMMARY OF RIKA'S LANGUAGE USE

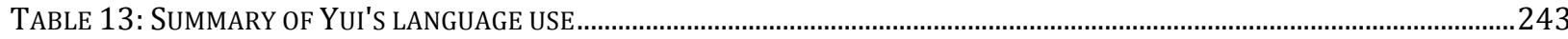




\section{Chapter 1. Learning to write and reason: Introduction}

Learning academic writing in an English as a Foreign Language (EFL) context presents a myriad of challenges to learners. Many of these challenges relate to the influences of their socio-cultural backgrounds on the written academic literacy they are attempting to achieve. One major issue for Japanese university students is that writing academic English requires a writer-responsible individualistic voice or writer identity (requiring writers to defend their own position) that is different from the more reader-responsible and collectivist voice (making a claim representative of many individuals speaking as one) used when writing in Japanese. When Japanese university students then choose to take on English-medium higher education overseas, this is a crucial consideration they need to make in order to meet the writing expectations of their university teachers.

\subsection{Origin and Rationale of the study}

As a young ESL university teacher at the University of Sydney, I tended to take note of the developments of the Japanese students in the program since I had just spent the previous three years teaching English in Japan. I noticed a trend with the Japanese students in their inability to pass the essay part of the in-house entrance exam to become matriculated students. Though the students seemed able to think critically about their argumentative essay topic, they were unable to display that thinking in the kind of formulaic essay the examiners were expecting. When exploring the research conducted with Japanese students at the time, which mostly focused on Japanese students in overseas English-medium settings, the fundamental question remained: What happens in their English writing classes before they leave Japan?

The purpose of this study is to explore the experiences of Japanese university students learning to write academic English in Japan in order to answer the question: What happens in an English writing class at a Japanese university before the student goes overseas for studies in English? The study follows for one year sixteen students who were enrolled in one of six different English Composition courses at a university in Japan that is known for its English language education and for sending students overseas for studies in English. As the level of English proficiency for these students is considerably higher than the average 
university student in Japan (because the department places very high priority for acceptance to the program on English language ability), it is important to note that it is not the intention of this study that the outcome be generalized to Japanese higher education as a whole. However, the research demonstrated that there were extreme inconsistencies between teachers' approaches and students' reception of those approaches. This is a point to which Japanese higher education in general should pay increased attention.

The relevance of this study today is that Japanese higher education is currently in flux. The Ministry of Education is seeking to create more international appeal through an increase in English language programs and content courses. This development is due to the decreasing numbers of Japanese students as the birthrate continues to decline. In the past decade national universities have gone corporate and private universities are competing with them for government funding, leading to more interest in the development of globallyaccepted English language education based programs.

This in-depth longitudinal qualitative study utilizes a combination of analytical frameworks in its investigation of English academic writing education in a Japanese university. This study serves to reveal both significant strengths and limitations in students' and teachers' approaches to English writing education at a Japanese university. It has both pedagogical implications and implications for research across cultures.

The specific focus of this study is on the students' learning to write critically in terms of developing critical argument and establishing writer identity. This specific focus was chosen because the writing required of them in their English-medium education overseas tends to be dependent on being able to display critical thinking skills in mostly analytical/argumentative essays in which the student writers must take a clear stance on a topic and develop it through critical analysis of information.

\subsection{Definitions}

The three key terms of socio-cultural position (within social constructivist theory), writer identity and critical argument underpin this study. Thus, I will now briefly define each of the key terms and their relationship to each other. 
Socio-cultural position is based on social constructivism theory, which stipulates that in social settings an individual's knowledge is constructed in groups; individuals learn from each other and for each other, and collaboratively develop shared meanings (Vygotsky, 1978). A student's socio-cultural position or identity in a writing classroom is the foundation he or she has on which to construct knowledge and is based on various factors including the classroom itself, the interpersonal contexts in the classroom, the student's purposes for being there, and her or his personal (social and cultural) background. This thesis utilizes this fundamental definition of socio-cultural positioning in order to examine individual development within a shared learning environment.

Writer identity or a writer's voice in academic writing is the writer's presence in the textreferring to the different ways the writer applies personal perception in a particular context. This is made apparent through the use of certain language, such as personal pronouns, and also through evaluation and assertion. In this thesis, writer identity is the basis for the analytical frameworks as it is in identifying the students' identities that we can better understand how their socio-cultural positioning affects their approaches to writing.

Critical argument is an argument in which the writer has critically evaluated and analyzed the topic in order to establish a clear position on it. In developing a critical argument, the writer must present the problem and provide a thesis, evaluate and assess the validity of relevant supporting and counter-evidence, and draw a conclusion. It is important to clarify the use of the word critical in this thesis, as there has been considerable debate over the use and application of this word. The term critical as used here utilizes the polysemic perspective of critical as critique because it refers to criticism as well as evaluation. This perspective allows for semantic flexibility as the current study has an intercultural context in which there may be multiple interpretations dependent on the context of discussions using the term.

\subsection{Thesis chapter outline}

The next chapter, chapter 2 , provides a review of relevant literature focusing on issues in second language (L2) writing education both in "inner circle" or "norm-providing" countries (Kachru, 1992) and in Japan, an "expanding circle" or "norm-dependent" country, 
in order to show that although there are generally accepted "inner circle" movements in the development of L2 writing education, these movements have been taken on in varying degrees outside that circle. This has culminated in a situation where a hodge-podge of approaches in L2 education are employed, ranging from the grammar-translation methods that focus on accuracy, to process writing that focuses on fluency, to post-process writing methods that focus on critical thinking skills. In between the review of the norm-providing and norm-dependant contexts of L2 writing education, I have inserted a section that examines contrastive rhetoric of Japanese and English in order to point out some fundamental differences between Japanese and English that suggest consideration of them is necessary in advancing English writing pedagogy in Japan. It is in this section that I raise the issue of Japanese students' critical thinking skills (to be defined in section 2.2.1), as there has been some debate on both the existence of it as well as strategies teachers take to elicit it. This then concludes with a focus on how these issues led me to my research questions.

Chapter 3, entitled "Theoretical framework of the study", continues the review of the literature, building on the background of EFL education in Japan with a specific focus on analyzing the fundamental theories of the study. The theories highlighted in this chapter include: socio-cultural theory and how it pertains to social constructivism in academic writing research, identity construction theory, and critical argument theory. The chapter first discusses the importance of the focus of this study on critical argument and writer identity, followed by an analysis of social constructivism as a fundamental philosophy to the study. The analysis first looks at social constructivism in education and identity construction, then more specifically in academic writing research. This leads to an analysis of the particular constructs of social constructivism my study draws on. The devising of the theoretical framework is then explained with a diagram provided to show the connections of theories functioning together in the study. This is followed by analyses of the phenomena of constructing cultural and academic writer identities, as well as developing critical argument in L2 writing, which includes a second diagram showing the process involved in that development. Finally, an examination is provided of critical writing as mediated action, i.e. a mode through which meaning is made, looking especially at the steps 
of the writing process that involve influence from peers and teachers. This examination is made in order to further develop the analysis of the development of critical thinking skills in L2 writing education.

Having examined the literature-based theory behind the study in chapters 2 and 3, chapter 4, entitled "Plans and paradigms: The methodological framework of the study," provides an examination of the research paradigms involved. I investigate the theory behind the methods and particular frameworks adapted for and used in conducting a qualitative embedded case study research project. Having established the rationale behind the approach, I then provide an outline and discussion of the validity of the data collection methods and analytical frameworks I used to answer the research questions. As a qualitative study, the three data collection methods including observations, students' written texts, and interviews are typical of studies in this area. It is important to maintain consistency with other qualitative studies in order to sufficiently build on previous studies in the area. However, the analytical frameworks used for the observation data and students' written texts were adapted from multiple sources in order to maintain a focus on identity and critical thinking. After providing a description of the ethical considerations of the study, the chapter then concludes with some additional comments on the implications of the methods and frameworks used.

Chapter 5 provides a description of the setting and participants, including the theory and basis for the selection process. In order to help maintain anonymity of the participants, I offer only a brief description of the university where the study took place. I then provide an examination of grounded theory and the different sampling approaches I used in selecting the participants. The teacher participants are first briefly described followed by a detailed description of all sixteen student participants including their educational background and plans for study abroad or post-graduate studies in English.

For the analysis of the observation data presented in chapters 6 and 7, a qualitative framework proposed by Ivanič (2004) for adaptation was used in conjunction with ideas presented by Hyland (2002a; 2005). Ivanič's "Discourses of Writing” framework was based on students' writing in English as an L1, but Ivanič proposes the adaptation of the 
framework for studies held in English L2 settings. Ivanič's framework was ideal in that I was able to take advantage of it by incorporating Hyland's ideas about the relationship between classroom practices and the effect they have on students' writer identities, especially when writing arguments. The result was a framework that focuses on two areas: 1) the students' understanding of audience, and 2) the students' development of their thesis. These two areas of focus effectively covered the instances where classroom practices intersected with students' developing critical argument and establishing writer identity. In addition to the observation data, data from the interviews were used to further support the analysis.

The analysis of the students' written texts is presented in chapter 8. For this analysis, Appraisal Theory from Systemic Functional Linguistics (Martin, 2000) was used to provide an analytical framework as a basis, with additional indicators for the analysis taken from Clark and Ivanič's (1997) idea of the three possible selves in academic writing, and Casanave's (2002) six writing strategies. Combining these three concepts provided particular insight to students' decisions made in producing the written text. Especially since not all students were required to write argumentative papers, being able to identify the selves and writing strategies used helped to better explain the syntactic choices identified by applying Appraisal Theory, and to be able to comment on the students' display of critical thinking.

In chapter 9, the research questions are more directly answered, with careful consideration given to both the underlying theories and data collected for the study. In responding to the research questions, the originality of the study is emphasized in the way it fills the gap and extends research in L2 composition studies.

In the conclusion, presented in chapter 10, I will first summarize the main findings, significance and contribution of the study. This is followed by a discussion of the limitations of the study as well as the pedagogical concerns and considerations for future research in this area. Finally I offer an evaluation of the future effect of the current study. 


\subsection{Final Comments}

Concerns about university students' critical writing ability were recently raised by the book Academically Adrift: Limited Learning on College Campuses (Arum \& Roksa, 2011) in which the results of a study conducted with 2,000 students in the US over their complete four years (2005-2009) of undergraduate studies revealed that $45 \%$ of those students demonstrated "no significant gains in critical thinking, analytical reasoning, and writing skills" during the first two years of college (p.36). In these key areas, 36\% showed no improvement by the time they graduated. Much of the blame is placed on the lack of academic rigor of the courses offered ${ }^{1}$. Since much of the English language writing education (curriculum and pedagogy) happening in East Asian contexts is based on American models, this certainly raises concerns about the direction of university writing education outside the US as well. It also raises concerns about the kinds of preparation university students outside the US need to make with regard to their academic writing skills before heading to exchange or postgraduate studies there. Now it seems, more than ever, we need to try to determine what it is university academic writing is really about, especially with regard to the role it plays in the development of students' critical thinking ability.

It is also important to note that there is some obvious concern in a study conducted by a Western researcher that focuses on the social and cultural positions of Japanese students and Western and Japanese teachers of English in Japan. The issue is that from an objectivist/positivist point of view—that all reality is objective and external to the mind and that knowledge is reliably based on observed objects and events-my own social and cultural position could potentially invalidate my study. But from a constructivist standpoint-to discover the ways that individuals and groups create their perceived reality, my position should rather be seen to inform the study. My position as a Western researcher and native user of the English language is quite different to the students' position as Japanese learners attempting to cross culture and display critical thinking in

\footnotetext{
${ }^{1}$ Although Arum \& Roksa's study did not focus specifically on writing courses, they concluded that the skills undergraduate students were lacking in general were writing and reasoning.
} 
their writing of English, a foreign language. Therefore, culture and language barriers became a large part of the consideration of the study, generating invaluable discussion of the conflicts that arise for both students and teachers in the EFL education setting. 


\section{Chapter 2. Individualized voices and critical thinking: The literature review}

In this chapter I will discuss literature in conjunction with the ideas presented in chapter 1, with a particular focus on what the literature says about the problems Japanese university students face in learning critical argument in their English academic writing courses. The chapter is divided into three sections: first, a brief overview of English L2 writing education in general is given. This is followed by an analysis of Japanese to English contrastive rhetoric in order to highlight the key challenges for Japanese writers of academic EFL writing. Finally a synopsis of EFL writing in Japan is provided to examine the context in which the study is situated.

In presenting the review of literature in these three particular sections, my intention is to show that the "inner circle" developments in English L2 writing education have not been considered carefully in Japan due to the rhetorical differences between Japanese and English, and that this may be the cause for the inconsistency of approaches currently taken in EFL writing education in Japan. To clarify, Japan is a country located in what Kachru (1992) described as the "expanding circle." This is the outermost of three concentric circles of English: the inner circle is comprised of the countries where English is the native language spoken, and where ESL is taught (US, UK, etc.); the outer circle includes countries where English is not the native language, yet it holds important historical value and has official status in certain arenas (India, The Philippines, Kenya, etc.); and the expanding circle, which includes most of the rest of the world where English has no official status but is still used widely, and where EFL is taught (most of Europe, East Asia, Egypt, etc.).

Another significant distinction made in terms of the variety of practice of English education was made by Holliday (1994), using the acronyms BANA and TESEP to differentiate between the mainstream English language teaching discourse in native-English settings in Britain, Australasia, and North America (BANA) and the English language teaching discourse in educational settings at the Tertiary, Secondary and Primary (TESEP) levels throughout the world. Holliday raised the issue that there were considerable differences in the discourse and the often-difficult circumstances found in classrooms. An important 
discussion regarding this issue is the misapplication of such methodologies first described by West (1960) with his distinction of Teaching in Difficult Circumstances (TiDC). These distinctions made by West and Holliday emphasized the fact that the mainstream English language teaching methodologies were based on ESL taught in multilingual, intensive settings with a small number of students and trained, native-speaking teachers. Outside this context, ESL and EFL practices were used in very different circumstances. EFL was often taught in monolingual settings under the pressure of passing examinations, with less frequent class meetings, larger classes and uncertain teacher training or proficiency. The distinctions were made to encourage skepticism about monolithic Western methods and to urge researchers and teachers to consider alternatives to the belief that BANA products and ideas were the only options in English language teaching regardless of the context (Smith, 2010).

One final clarification to be made regarding the issue of contrasting varieties of practice in different cultures is that of the impact of Confucianism on English language education in East Asia. Turner (2011) discusses the dangers of Western hegemony in interpreting the impact as detrimental to East Asian students ability to learn English, and encourages researchers and teachers to take a more "globally neutral" approach (p.109). She refers to Ballard and Clanchy (1991) who described Western academic cultures as "extending knowledge" and Confucian cultures (including Japanese) as "conserving knowledge." What this distinction implies is that Western cultures are inherently forward thinking, leaving Confucian cultures behind. Turner refers to Kubota's (1999) criticism of this distinction, labeling it "orientalist." The problem with this criticism, Turner points out, is that it suggests that the perspective is somehow universally accepted. Even in doing so, there is no reason to assume that a "conserving knowledge" approach should be seen as negative. Turner encourages those involved in this discussion to remember the importance of understanding the differences between the Confucian thinking in eastern educational approaches and the embedded critical thinking in Western critical approaches and not to place higher value of one over the other. This is particularly important with the increase of globalization in higher education. The issue of the Confucian influence on Japanese learners will be discussed further in section 2.2.2 on Japanese students' critical thinking. 
In the construction of the second and third parts of this chapter, some of the organization is based on Aspinall's (2003) five major reasons why EFL education in Japan has been unsuccessful, namely that:

1. There is a great linguistic disparity between Indo-European languages, such as English, and Japanese, which is an Altaic language.

2. There is a lack of real need for English in a monoglottal society such as Japan.

3. The predominant ELT methodology has been grammar-translation, which is not an effective way to teach communicative skills.

4. The culture of the language classroom in Japan precludes effective language learning.

5. There is an exotic and fashionable image of English which emphasizes entertainment value rather than the hard work necessary for effective language learning.

Aspinall is a professor of political science at Shiga University (international), near Kyoto, Japan. His criticism of EFL education in Japan was given similar assessment by Reesor (2003), who taught at a university in Japan from 2001-2004. Reesor's criticisms have been further discussed and confirmed by yet another university teacher in Japan (Rapley, 2008; 2010). The first of Aspinall's reasons will be discussed in relation to issues of Japanese to English contrastive rhetoric, while reasons 2-4 will be discussed in the last part on EFL writing in Japan. The fifth reason is one that will not be addressed in this study and is discussed later for consideration in future studies in this area.

\subsection{English L2 writing education: A brief overview}

In order to gain a comprehensive understanding of English writing in Japan, it is necessary to first examine relevant literature on the history of teaching English as a second or foreign language. This section will provide a brief overview of what the literature offers on English L2 instruction methodologies-both past and present-including grammar-translation, the 
product approach, the process approach, post process and genre-based writing instruction, all of which still resonate in English L2 writing teaching practices in Japan today.

\subsubsection{Grammar-translation}

Up until the 1970s, the predominant method of ESL instruction in inner-circle, BANA contexts was the grammar-translation method, which entailed having a student directly translate sentences from his or her first language (L1) into the target language (L2) (Richard \& Rodgers, 2001). Fotos (2005) described the grammar-translation method as consisting of nothing more than exercises in handwriting, writing dictation and writing the answers to reading and grammar questions, but this system did allow teachers to strictly control the course content. A significant difficulty with this method is that it required students to already have a comprehensive understanding of their L1's grammar structures, which many did not.

\subsubsection{The product approach}

At the same time the grammar-translation method was being used in language teaching, "controlled composition" (Matsuda, 2003) or "the product approach" began making headway into L2 writing education. The product approach prescribed models or templates, getting students to practice particular grammatical and syntactical patterns often focusing on "the arrangement of sentences into paragraphs" (Hedgcock, 2005, p.604). According to Hedgcock (2005), as the role that an individual's L1 played in his or her ability to acquire strong L2 composition and writing skills rapidly became clear, teachers began to move from using the formulaic, direct grammar-translation method to guided writing in which they encouraged their students to create short "discourse" pieces through a sequential focus on model, form, and duplication. Much of the work that students completed still maintained its focus on correctness and direct translation and required students to answer questions by combining pre-formulated sentences-a technique that resulted in the construction of discourse (Reid, 2001). This approach was found to be stultifying for students, ignoring the processes involved in creating the product (Eschholz, 1980). 


\subsubsection{The process approach}

Instruction in L2 writing began to make significant changes in the 1970s according to Leki (2000). ESL writing instructors were growing dissatisfied with the focus on grammar and correctness in the product approach and wanted to put more emphasis on the communicative processes of reaching the end product, i.e. the stages of pre-writing or brainstorming, outlining, drafting, revising and editing. This process approach emphasized the importance of writing to communicate a message to a reader, as opposed to writing for examination purposes. They turned to the emerging L1 composition pedagogy of the process approach. This shift in the ESL curriculum was mirrored in the native English classroom, where the building of strong communication skills became the focus of many curricula and resulted in many policy-based changes (Caroll, 1997). This change also marked the beginning of the "process revolution", a restructuring that would result in the recognition of pre-writing activities, such as brainstorming and outlining, and revisions as a formal part of the writing process (White \& Arndt, 1991; Tsui, 1996; Rinnert \& Kobayashi, 2001). ESL students were expected to acknowledge feedback from various sources, to discuss their writing with their teachers, tutors and peers and to revise their papers based on the commentary received (Duppenthaler, 2002).

The process revolution coincided with the rise of communicative language teaching (CLT), in which L1 English university composition classes began to focus on the writing process as a form of self-discovery with an emphasis on creativity and fluency (Zamel, 1982; Hedgcock, 2005) in the form of what was called "writer-based prose" (Flower, 1979). The concept also addressed "reader-based prose," which was described by Flower (1979) as "an alternative to the 'think it/say it model' [in which] effective writers do not simply express thought but transform it in certain complex but describable ways for the needs of the reader" (p.19). Through a focus on reader-based prose, writing was viewed as an act of internalizing expression in verbal form to oneself rather than externalizing it to an outside

reader in written form. This had implications for the writing process coming to be understood as a way of knowing rather than telling by viewing writing as an act of internal discovery (McCrimmon, 1980). 
ESL classes soon followed suit after a 1981 study in which Elbow claimed that fostering the development of an "individualized voice" could help cultivate fluency, momentum and strength in students' writing. This new focus encouraged students to think in their L2, thus weaning them off their reliance on direct translation and simple sentence combination processes (Raimes, 1983). Bereiter \& Scardamalia (1987) argued that the focus on writerbased prose was in fact a form of knowledge telling that functioned more as an alternative writing strategy that, although highly efficient in students' output, bypassed the need for a goal-directed knowledge search and did not lead students toward an understanding of more complex planning. Citing Bereiter and Scardamalia (1987), Leki and Carson (1994) suggested that writing that comes from complex planning is a kind of "knowledgetransforming" writing that may facilitate learning better than the more traditional "knowledge-telling" learning in which students report what they know or have experienced without building and reflecting upon it or using it for a real purpose. Although the newly implemented process in writer-based prose did eventually lead to the integration of a more supportive student-teacher editing and revision process, it still lacked a true prioritization of identity and creativity because it forced students to fit their writing into a pre-fabricated, structural mold (Byrd, 2005) and favored communicative fluency over accuracy in the L2.

The shift to beliefs that language education should be communicative also led to a special focus on writer identity and academic writing as a communicative social act (Rinnert \& Kobayashi, 2001). Although the communicative approach was famously criticized by Swan (1985a; 1985b) for ignoring the socio-cultural backgrounds of the learners in their L1, Communicative Language Teaching (CLT) continues to strongly influence language teaching around the world. While CLT originally emphasized speaking and listening skills, it was later applied to writing that encouraged students to work towards establishing a clear voice and position in relation to their audience and context, the cultivation of an "authorial identity" (Clark \& Ivanič, 1997; Ivanič \& Camps, 2001). This type of writingwith a focus on the audience-became known as "communicative writing" (Carter \& Nunan, 2001).

Communicative writing not only introduced the idea that socio-cultural environments can have an impact on a student's ability to write effectively in her or his L2 (Atkinson, 2003; 
Lantolf \& Thorne, 2006), but also paved the way for the introduction of critical thinking as a part of the writing process, which would combine the process and product approaches with the authorial-voice-inducing CLT method (Atkinson, 2003; Matsuda, 2003; Kent, 2003). This came to be known as "post-process" writing.

\subsubsection{Post-process writing}

First introduced in 1994 and attributed to Trimbur (1994), the term post-process was created after writing theorists realized the necessity of acknowledging the social nature of writing (along the lines of Swan's 1985 argument) and began to embrace the idea that writing is a public, interpretive and situated process (Kent, 2003). While researchers such as Atkinson (2003) have incorporated this term into their studies of L2 writing, others such as Matsuda (2003) insist that the term itself can be misinterpreted as a rejection of the writing process. In a 2008 study, Heard suggests that these conflicting viewpoints may have served to reduce the potential of the post-process methodology to overturn previously accepted theories and concludes that post-process writing should be implemented and integrated into existing pedagogical methods at the university level.

As part of the post-process movement, research in critical perspectives in L2 writing has also debated as to whether critical theory has caused the field of academic writing to be overwhelmed by discussions of politics and ideology (e.g. Benesch, 2001; Canagarajah, 2002). In a discussion on ideological, political and identity issues in L2 writing in Leki, Cumming and Silva's (2008) A Synthesis of Research on Second Language Writing in English, it is explained that from 1980 to 2005, L2 writing research focused on English "(and its current academic writing preferences) and on the role of a critical perspective in L2 writing instruction" (p.60). Meanwhile, L1 writing education was characterized as ideological, while L2 writing education was characterized as pragmatic (Santos, 1992). Benesch (1993) argues that with or without politics, all teaching is ideological, and issues of power and social justice have been ignored in the past in L2 teaching. In critical pedagogy, Benesch (2001) explains, "the guiding principle is that the curriculum is an ongoing negotiation based on the interests, desires, and needs of the students" (p.71). Based on studies from the 1990s and 2000s, this ongoing negotiation goes beyond needs analysis and has been related to issues of 'the power dimension inherent in students' academic lives 
and the possibility of helping students negotiate that power, critical literacy, and the negotiation of competing discourses inherent in the integration of multilingual literacies" (Leki, Cumming \& Silva, 2008, p.62). Canagarajah (2002) explains that this negotiation can be achieved through critical literacy, as it is what helps bring writing matters into sharper focus, for both the students and teachers. He continues to explain that the label critical "alerts us to the power-and dangers-of literacy... It develops an attitude and a perspective that enable us to see some of the hidden components of text construction and the subtler ramifications of writing" (Canagarajah, 2002, p.1).

\subsubsection{Other approaches to writing instruction}

While process-writing models have dominated English L2 writing instruction for the past several decades, they have been contested by more socially oriented views of writing that put a greater focus on learners and their needs (Hyland, 2003). This has led to more attention being given to genre approaches, which utilize the product approach while complementing process views. They also emphasize the role of language in written communication while maintaining the idea that writing is a social activity. Increased attention has been given to genre-based approaches over the last decade (Hyland, 2007). Genre approaches see writing as purposeful, and respond to the social aspects of the writing context (Hyland, 2002a). As genre-based writing instruction (i.e. teaching writing according to specific types such as expository, evaluative, argumentative, etc.) developed, it has come to be contested that there is only one approach to this method, and that there are, in fact, at least three or four approaches that have implications for L2 writing pedagogy (Johns, 2011).

Hyland (2008) described three theoretical writing approaches that may be used in genrebased course design. The first, a "text-oriented" approach, is based on the key idea of genre and emphasizes writing forms using modeling and accuracy of language use. He explains, "while genre teaching means attending to grammar, this is not the old disembodied grammar of the writing-as-object approach but a resource for producing texts" (Hyland, 2008, p.96). To encourage students to use the grammar rules in order to write well-formed, appropriate texts, he proposes the "teaching-learning cycle" that shows genre learning as five linked stages including: 1) building the context, 2) modeling and deconstructing the 
text, 3) joint construction of the text, 4) independent construction of the text, and 5) linking related texts (Hyland, 2008, p.96). Genre teaching has received criticisms for forcing students to fit their writing into existing models, therefore "stifling" creativity, but Hyland (2008) suggests that by accepting genres as establishing expected patterns rather than "dictating" rules, genre teaching provides choices for writers to make meaning (p.98).

The second is a "writer-oriented" approach, which focuses on the writer's identity and thought processes rather than the product or text. This approach understands writing as expressive or cognitive and sees the writing process more as problem solving than an act of communicating. It is concerned with writers' development of knowledge of topics in order to write effectively. Hyland (2008, p.101) expresses two fundamental problems with the emphasis on "what people think about when they write rather than the language they need to do it." One is that the process is decontextualized, focusing on the "writer as an isolated individual struggling to express personal meanings" (Hyland, 2008, p.101). The other is that the discovery approach denies student writers explicit language when they need it.

Finally, a "reader-oriented" approach "emphasizes the interaction between writers and readers" (Hyland, 2008, p.103). The focus is on writing for the purposes that a reader will be able to understand it. This approach stresses the importance of some kind of a community-discourse, academic, classroom, etc. For teachers, Hyland points out that it is important to "be clear about the purposes, genres and readers" students need for written communication. The reader-oriented approach requires an understanding of what features are used to communicate with different readers. Teachers with this understanding can help student writers to target their readers within certain contexts and raise their "rhetorical consciousness" (Hyland, 2008, p.105).

\subsection{Contrastive rhetoric: Japanese to English}

This section will focus on the first of the five major reasons raised by Aspinall (2003) as to why EFL education has been unsuccessful in Japan, by examining the great linguistic disparity between Indo-European languages, such as English, and Japanese, which is an Altaic language. This will be done through a discussion of Japanese to English contrastive rhetoric, which will further highlight the challenges faced by Japanese students, 
particularly in displaying critical thinking when writing in English in a foreign language context.

In the findings of a self-initiated 1966 study, researcher Robert Kaplan established the idea that students whose L1 grammatical and organizational structures differ greatly from those of their chosen L2 would find language acquisition more challenging. While reflecting on Asian learners of English, he remarked that, "Some Oriental writing...is marked by what may be called an approach by indirection" and portrays the "Oriental" writing style as a spiral (Kaplan, 1966, p.17). However, despite his having shed light on a major problem inherent in East Asian grammar-translation methodology, researchers of Japanese to English contrastive rhetoric such as Noor (2001) and Stapleton (2001) criticize Kaplan's 1966 findings for overgeneralizing. Due to such criticism and further research into writing styles in Asian languages, in 1987 Kaplan revised his assessment of "Oriental" writing style to take a less critical stance of its structure.

This section will first provide an analysis of the structural differences between Japanese and English in order to interpret the challenges Japanese writers of English really face. This is followed by an analysis of the literature related to Japanese students' critical thinking and how that affects their writing. The implications of these issues will be discussed as characteristics inherent in Japanese that require further analysis.

\subsubsection{Structural differences between English and Japanese}

As mentioned in the introduction to this section, Kaplan noted in his oft-criticized 1966 study about second language acquisition that the grammatical structures of many East Asian languages, including Japanese, differ greatly from those generally used in English. This reasoning lends itself to supporting the persistence of grammar-translation methodologies in Japanese L2 writing instruction, which will be discussed in more detail in section 2.3.3.

Some Japanese researchers of Japanese to English contrastive rhetoric have claimed that Japanese writing is characterized by its inductive style, i.e. not directly stating the thesis in the introduction, but rather hinting at it. The inductive style has been described as manifesting itself in the classical form of ki-shou-ten-ketsu (Hirose, 1998; Kubota, 1997; 
1998), which forces readers to draw their own conclusions based on the information given, a characteristic known as "reader responsibility" (Kubota, 1997). Though most researchers agree on the quasi-inductive (Hinds, 1990) nature of ki-shou-ten-ketsu, no researcher has come up with an unchallengeable definition of the style (Kubota, 1997; Hirose, 2005).

The ten of the essay, sometimes referred to as a "topic shift," is what sets the Japanese writing style apart from the generally accepted English language essay format (Hinds, 1983), in which it may be recognized as opposing argument. In a Japanese essay, as proposed by Takemata (1976, cited by Hinds in 1983), a writer begins his or her argument in the $k i$ portion of their composition, just as writers of essays in English would present their thesis statement at the beginning of an essay. When writing in English, the shou, or topical development phase, is represented through the presentation of commentary and evidence-generally known as body paragraphs-and occurs in alternation with ten-the transitional phase (Kubota, 1997). Both English and Japanese essays end with conclusions - the ketsu (Hinds, 1983). Hinds explained that in this type of Japanese writing "the main ideas do not appear until the end and that the paragraphs before the main ideas do not constitute the reasons or evidence for the main ideas" (Kubota, 1998, p. 70). The type of inductive writing style described here goes against the generally preferred deductive writing style for native English speakers, which is characterized by providing the thesis in the introduction of a broad topic that is then supported or defended using evidence (Noor, 2001), but this is not the preference of only native English speakers. Kubota (1992) described one study that showed Japanese professors preferred expository and persuasive essays to be deductive, and that good Japanese essays share a similar writing structure to well written English essays (Kubota, 1997, p. 461).

According to researchers of socio-cultural theory (Lantolf \& Thorne, 2006), Japanese writers are influenced by strongly maintained principles (Atkinson, 2003; Casanave, 2002) such as aimai (ambiguity) and haragei, literally translated as "force of personality," as defined by Davies and Ikeno (2002). These ideas have played a large role in defining the way that many Japanese people act, speak and write. Similar to aimai, haragei is often used to avoid direct confrontation either verbal or written because it does not force the speaker to voice explicit, potentially offensive opinions and allows-if not forces-the target party 
to inductively draw its own conclusions based on the context of the situation. Hinds (1985) suggests that while writing in English often assigns the task of effectively conveying one's meaning to the writer, Japanese writers employ "reader-responsible rhetoric", which instead puts the task of finding meaning on the reader (Noor, 2001)-further evidence of aimai and haragei in Japanese writing. According to Kubota (1997; 1998), McCagg criticized this idea in 1996; after re-analyzing the content used in Hinds' 1987 study, he concluded, "Japanese texts do not generally require greater cognitive effort from readers for comprehension than English texts do, as long as the reader and the writer share the same cultural and linguistic knowledge" (Kubota, 1998, p.70).

Though consideration of socio-cultural theory in EFL and ESL teaching is relatively recent, this concept, introduced in the late 1990s, has helped academic theorists understand the intimate bond between L2 writers and their environment (Rinnert \& Kobayashi, 2001; Casanave, 2003). Due to the fact that many writers receive no formal writing instruction during their schooling (Casanave, 2002; Hirose, 2005), it becomes even more important to acknowledge this inherent connection, especially in a genre-based writing curriculum because with no formal instruction on writing forms, there is little or no basis on which to build students' understanding of the various writing genres. Hirose, in her 2005 study, concluded that students who are not educated in the guidelines of academic writing will, when asked to pen a composition, merely transcribe spoken, colloquial dialogue created either initially in Japanese and directly translated into English, strictly adhering to the grammar-translation method, or written conversationally in English. L2 students will often draw from their past experiences or the sample sentences provided to them while preparing for essay exams (Casanave, 2003). They will sometimes attempt to make their writing sound native by mimicking features from their sources (Masao, 1976) or copying the text directly (Casanave, 2002)-tendencies that often result in the inhibition or complete loss of writer voice and identity. Ivanič (1998) raises the questions:

What can be attributed to the author, what to the reader, and what to the community? Is copying from another writer a positive act, a way of showing agreement and allegiance? A way of taking to yourself the values, ideas and discourses of your discipline? Or is it a sin comparable to forgery, known as plagiarism? (p.4) 
Such copying is noted when students use quotes instead of paraphrasing or summarizing, and have difficulty integrating those quotes. Students who successfully integrate source information may have done so by mimicking their sources.

\subsubsection{Japanese students' critical thinking}

Some researchers also argue that Japanese students may not be able to write critically, citing traditions of collectivist and hierarchical traditions that inherently discourage students from developing a distinct identity or voice in their writing (Atkinson, 1997). Some academics such as Jane Barnes Mack-Cozzo, who taught English at universities in Japan for 12 years, go even further, emphasizing the idea that students are not only discouraged from writing explicitly but also are steered away from "creative original thinking... for it goes against the wa (harmony)" (Mack-Cozzo, 2002, p.47). However, other researchers (e.g. Stapleton, 2002a; Kubota, 1999) vehemently argue against this stereotype, insisting that Japanese students take distinctly Japanese approaches to critical writing, and are therefore no less critical.

It is important in reference to the argument about Japanese students' critical thinking to provide a working definition of critical thinking. I refer to Ennis's (1998) definition from his article Is Critical Thinking Culturally Biased? in which he explains "critical thinking is thinking that is reasonable and reflective, and is focused on what to believe or do" (p.16). He goes on to explain that critical thinking is not limited to individuals, and suggests that as long as "group thinking" exists, it can be done critically. With this understanding, the goal of critical thinking, either individual or collective, then is to make reasonable decisions by seeking reasons and alternatives. The importance of critical thinking is stressed differently in East Asian cultures where social practices focus on collective concordance and compliance (Atkinson, 2003). It has been suggested that this interpretation of an inability to think critically may be due to the structure of the Japanese education system (Lee-Cunin, 2005), which has maintained somewhat Confucian ideals that dictated the importance of the relationship between master and pupil and decreed that a student was never to question his or her master (Stapleton, 1995). 
However, it may be that the impact of Confucian ideals has been misinterpreted by the West. Turner (2011) points out that as the number of students from East Asian countries such as China, Japan, Korea and Taiwan has increased in the UK, their behavior has served as a point of difference between Eastern and Western students. She argues that this difference has become entrenched, but that Western interpretations, maintaining the negative stereotypes of "passive" or "silent" or "uncritical" East Asian students, are no longer appropriate (p.97). Turner explains that Western instructors see the passive nature or silence of East Asian students in classrooms as resistance to speaking in class, but she argues that there are culturally weighted differences regarding both silence and listening, which are seen as proactive. While Western approaches may emphasize "learning through speaking," this is an activity traditionally frowned upon by East Asian students who would prefer to follow the tutor who should serve as an exemplar or model demonstrating what should be done. It is a different way of learning (opposite to the Western approach of tutor as facilitator), reflecting Confucian tradition in which students follow their tutor's lead on "their own individual journey of self-perfection" (Turner, 2011, p.161). Turner emphasizes the importance of recognizing and understanding both the conflict between, and transformative effects of these two approaches as intercultural interaction plays an increasingly significant role in the global cultural construction of international universities.

Turner (2011) goes on to explain how the "contrasting educational ideologies" of East and West place different value on critical thinking. Because "being critical is historically embedded in Western educational culture as a positive value," it requires an understanding of the "rhetoricity of being critical" (p.185). As East Asian students have not grown up with such rhetoricity, they may be seen in Western contexts as "uncritical." Traditionally it is important for a Japanese student in Japan to display an ability to listen and read without criticizing or evaluating, and rather than reinforcing opposition, to find harmony in a comparison of two opposing views. However, these actions are not in line with the goals of Western educational approaches. For such Japanese students, Turner explains that providing critique is more than "being critical," which may be understood as simply disagreeing (p.189). She emphasizes that being critical has "gone global as an educational issue" (p.192) and an understanding of "rhetorical exchange value" is of crucial importance, 
considering the strengths of both Eastern and Western interpretations of it, in order to avoid East Asian students seeing the Western understanding of being critical as superior.

The debate on Japanese students' critical thinking is difficult to conclude. Bruce Davidson, a long-time academic in Japanese universities and researcher of Japanese students' critical thinking asserts, "Japan paradoxically offers both an encouraging and an inhibiting environment for the development of critical thinking skills" (Davidson, 1995, para.3). According to an American student enrolled at Tokyo University, "Students were afraid to ask questions. They were afraid someone might ridicule them for not knowing the answer" (Taylor, 1983 in Davidson, 1995, para.8). A Japanese graduate student of Davidson's studying in the UK reflected this sentiment and stated, "even Japanese educational system doesn't encourage us to have our own ideas" (Davidson, 1995, para.10). Davidson (1995) takes this argument a step further and states that based on his own in-class studies, "Many people in Japan seem to have some difficulty discussing ideas or even in explaining them" (para.7). He argues that many Japanese students find the act of discussing or critiquing ideas difficult because "they do not have any opinions; and if they have opinions, they often cannot explain or justify them" (Davidson, 1995, para.7), thus further promoting the idea that Japanese students have an inability to think critically.

The arguments of both Atkinson (2002) and Davidson (1995) directly contradict Stapleton's (2001) study in which he took writing samples (of responses to provocative essays he wrote) from 45 undergraduate students in courses with the title "English Writing" in order to propose a model of assessment for critical thinking. In this study Stapleton concludes that, "participants demonstrated a fundamental understanding that all opinions require support" (p.526) and wrote with "individualized voices, which are closely related to critical thinking ability" (p. 534). Furthermore, students who lack background knowledge about or some level of familiarity with their assigned topic are less likely to demonstrate the ability to think critically due to "a lack of shared assumptions between the non-Asian researchers and their Japanese students" (Stapleton, 2001, p.530).

Therefore, despite past claims that cultural tendencies keep students from developing distinct critical voices in their writing, evidence of students' critical thinking abilities is 
becoming increasingly visible (Stapleton, 2002a; Kubota, 1999). These findings mirror those of Casanave's in her 2002 study. Here she reported that although the students of one particular ESL teacher sometimes encountered problems while attempting to complete the exercises they had been assigned, they also "wrote that they were learning to think critically" (Casanave, 2002, p.60). It would seem necessary that in order to write in a way that is acceptable to raters of English ability, one must first develop her or his critical thinking skills (Stapleton, 2002a; Casanave, 2002) and a good understanding of the strategies required to meet raters' expectations. Yuko, another one of Casanave's test subjects, admitted that she had little background in her chosen topic of International Relations, and consequently had a hard time processing and understanding the materials she had been provided with for her final paper (Casanave, 2002).

The main issues in the debate on Japanese students' critical thinking are often based on particular characteristics inherent in the Japanese language. As explained in the introduction to this section, Kaplan (1966) provided graphic depictions of various types of L1 writing styles and portrayed Japanese writing as a circular spiral of logic that spends the entirety of the essay alluding to a point but perhaps never explicitly stating it even by the end-a tendency that reflects the influence of aimai and haragei (Davies \& Ikeno, 2002)what Hinds (1990) referred to as a "delayed introduction of purpose" (Dyer \& Friederich, 2002, p.278. Historically, instruction in Japanese L1 writing concentrated on personal expression, requiring students to write about their feelings and personal experiences. Along this line, students also practiced kansobun, in which writers describe their impressions of assigned readings (Hirose \& Sasaki, 1994). In Dyer and Friederich's 2002 study on teaching autobiography in Japan, they cite Arai (2000), a Japanese professor of English in Tokyo, who in a criticism of the L1 writing process stated that "in Japanese writing instruction, the emphasis is placed on two elements, jiyu (freedom) and jibun no iken (one's own opinion): 'Students are encouraged to write down their ideas spontaneously, without worrying about such irrelevancies as organization, clarity, or logic'” (p. 6, cited in Dyer \& Friederich, 2002, p.278). This understanding of non-linear logic or random quality in Japanese essay writing is based on the history of essay writing. Indeed, 
the Japanese word for essay, zuihitsu, is translated as, "writing at random" (Dyer \& Friederich, 2002).

The idea that Japanese writing uses non-linear logic has prevailed in related publications over the decades. In Fox's (1994) book Listening to the World, she quoted a student in Japan on the vagueness of Japanese writing compared to English: "Japanese is more vague than English,' she [a Japanese student] tells me. 'It's supposed to be that way. You don't say what you mean right away. You don't criticize directly'" (Fox, 1994, p.8). However, this debate on non-linear logic in Japanese essay writing is concerned more with personal writing rather than expository writing. According to Dyer and Friederich (2002), Japanese personal writing is not all that different from English personal writing. Although university English composition instructors may believe that this aspect of Japanese writing is a handicap for Japanese students learning to write English academic essays, both Doi (1973) and Matsuda (2001) insist that this tendency should be viewed as a concept that enables Western theorists to develop a more comprehensive understanding of the Japanese individual and cultural psyche.

The issue of impersonal writing needs to be raised at this stage as a style Japanese student writers struggle with in expressing their thinking in their academic writing. In his article Opinions of identity in academic writing, Hyland (2002b) raises the issue about the "myth of impersonality" in academic writing. He explains that teachers requiring students to remove personal writing, deleting phrases such as I think or I believe, is an oversimplification of the issue. He explains that the differences between disciplines affect the ways people in those disciplines argue and express themselves. As learning to write at university often means taking on new identities, he suggests students need to learn how subject-specific writing differs, in order to provide them with "an awareness of the options that academic writing offers" (Hyland, 2002b, p.352).

The Japanese "habit" of writing inductively (Kubota, 1997) can also be attributed to the principle of kenkyo, which is literally defined as "modesty" (Davies \& Ikeno, 2002). Davies and Ikeno explain that kenkyo is important in Japanese culture because "Self assertiveness is more or less discouraged, while consideration for others is encouraged" (p. 143). This 
idea manifests itself in Japanese speech as well. While addressing an individual of a higher rank or social status than themselves, native Japanese speakers will add softeners to their speech or let their sentences trail off in order to avoid being perceived as overly assertive. This tendency may show itself in the writing of a Japanese student of English, particularly if he or she has not received formal instruction in writing academically (Hirose, 2005). Furthermore, Japanese mothers often emphasize to their children the importance of empathy and conformity, two characteristics that directly contradict the nature of critical thinking (Stapleton, 2001), and this tendency manifests itself in both the classroom and in students' writing. Davidson (1995) notes that his Japanese students were "often quick to drop a point they make in the face of disagreement. They will often immediately grant the validity of the opinion of the other party without challenging her reasoning" (Davidson, 1995, para.9). Thus, the notion of conformity, which is emphasized in Japanese society from a young age, may also impact on the level of argumentation and critical thinking in the students' writing in my study.

While socio-cultural tendencies and pre-formulated schemata often play a large role in the molding of an individual's writing style, it is also important to consider the implications of gender when studying the development of a particular writing style or tendency (Kubota, 2003). This importance is especially emphasized in the Japanese language where male and female speaking styles are clearly differentiated. Japanese speakers universally accept and employ humble tones and softeners as a way to show their respect for elders and higherranking individuals - for women, this often includes their husband and other men in their lives. This demarcation of status is not only verbal but also written-the kanji for husband is comprised of shu (master) and jin (person), while the kanji for wife is sometimes written as $k a$ (home) and nai (inside). Since the Tokugawa Shogunate and subsequent rise of the very paternally focused Confucianism, women have been both demoted and subordinated (Davies \& Ikeno, 2002) despite the passing of equal rights laws and the introduction of coeducational higher institutions (McVeigh, 2002). This cultural subjugation, although rapidly changing, is reflected in the words of the Japanese language itself; by nurture and socialization, Japanese spoken by females uses more softeners and is consequently more indirect than Japanese spoken by males, which is often very concise (Davies \& Ikeno, 2002). 
Though briefly mentioned by Kubota (2003), whether gender actively plays a role in the ability of Japanese students of English to communicate both concisely and effectively should be investigated further, due to the fact that many of the studies cited in this chapter were performed on test groups consisting primarily of female subjects.

This particular area of focus is crucial to this study. If Japanese students' critical thinking skills are not fostered in their university education, whether due to Confucian education ideals, Japanese "reader-responsible" rhetorical structures, or misinterpretations by Western instructors, this has serious implications regarding their ability to meet writing requirements in English-medium university studies overseas.

\subsection{EFL Writing in Japan}

Now that a general overview of English L2 writing pedagogy and Japanese to English contrastive rhetoric has been provided, it is necessary to apply these concepts in an analysis of EFL in Japan. In this section I will first briefly describe what the literature says on the history of EFL in Japan in order to address Aspinall's (2003) second reason why EFL education has been unsuccessful in Japan, which he states is due to a lack of a real need for English in a monoglottal society such as Japan. This section will also address Aspinall's third reason, that the predominant ELT methodology has been grammar-translation, which he states is not an effective way to teach communicative skills. This will be followed by a discussion of Aspinall's fourth reason, that the culture of the language classroom in Japan precludes effective language learning, which will be examined through an analysis of EFL at the pre-university level and then at the university level in Japan. Finally, I will examine the current approaches in EFL university writing courses in Japan.

\subsubsection{EFL in Japan: A brief history}

The first major consideration of EFL in Japan is the fact that, as Aspinall (2003) pointed out, there is no real need for English in Japan as Japanese is the only language necessary and most people only speak Japanese. This is often explained as a result of Japan's more than 200 years in isolation under leadership that resisted foreign influence until 1853, when the US finally initiated trade relations with Japan. At that time, English replaced Dutch as the major Western language studied in Japan. Interest in studying the English language 
dropped dramatically and was even discouraged during World War II, and then rose dramatically after the war in the proceeding decades as Japan's economic prosperity grew (Kubota, 1998, p.300). However, throughout these periods the interest in foreign culture has remained one of a separatist nature. Seargeant (2005) explains that this separatist nature can be observed through Japan's tourism industry, which essentially manipulates foreign cultures for Japanese consumption as it relates to the dochakuka principle-the absorption and adaptation of global forces into local cultures. This serves to explain the often-described "ornamental" function of English in modern Japan, which does not adhere to the generally understood implications of English as a Global Language (Seargeant, 2005). This has significant implications for the long history of the grammar-translation method used in ELT in Japan.

Aspinall (2003) asserts that the predominant ELT methodology in Japan has been grammar-translation. This raises a serious dilemma about the effectiveness of Japanese university students learning English communicative skills, including writing. While yakudoku (grammar-translation) dates back to the Nara period, when Japanese Buddhist scholars translated Chinese texts directly into Japanese (Henrichsen, 1989), English instruction was only introduced into Japanese schools after the ending of the country's selfimposed isolation as a part of the 1868 Meiji Restoration period. Implemented after a series of educational reforms that were passed between 1872-1890, English writing instruction functioned under the Confucian ideals that had become an inherent part of the Japanese education system under the rule of the Tokugawa Shogunate, when schooling had been based on Confucian principles of hierarchy. These principles dictated the importance of the relationship between master and pupil and decreed that a student was never to question his or her master (Moore \& Lamie, 1996)-ideas that are still vaguely present in the Japanese school system today.

Kubota (1997) notes the internationalization policies ushered in by the Meiji Restoration also resulted in an ideological shift in the writing sphere: The Sino-Japanese written kanbun style that pre-Meiji writers had adhered to was deemed outdated and was replaced by the "speech and writing correspondence" genbunicchi technique, popular amongst individuals educated in Western literature. The integration of this new approach resulted 
in the adoption of various English language grammar techniques into the Japanese language - additions included third-person pronouns, the use of commas and full stops and new conjunctions (Twine, 1991). By assimilating more of the grammar structures commonly used in English into Japanese, individuals were able to hone their direct translation skills. According to Kubota (1997), Morioka (1963) used Western composition theories to introduce a method of kompojishon (composition) to Japanese writing in order to "help Japanese writers produce texts in a structured manner" (Kubota, 1997, p.472). Morioka did this by focusing on ideas such as "the development of a theme, outline, paragraph, grammar, and usage of words and characters" (Kubota, 1997, p.472). However, despite Morioka's discussions about integrating Western writing styles into the Japanese essay, Japan has predominantly continued to teach direct translation in the EFL classroom (Yanabu, 1981), a tendency that can still be seen today in English textbooks-proof that Japan has yet to reach the process revolution.

\subsubsection{Pre-university EFL education in Japan}

Aspinall's (2003) claim that the culture of the language classroom in Japan precludes effective language learning has serious implications. With consideration of the historical influences on the language classroom including Confucian ideals, a Japanese separatist nature, and the anti-language-building assessment practices inherent in an exam culture, Japan's Ministry of Education decided to reform its English language education again in 1987. Much of this major reform centered on the implementation of the Japan Education and Teaching (JET) Program, after it was determined that native speakers of English were required in the English language classroom in order for students to be able to build on and practice effective oral communication skills (McConnell, 1996; Gorsuch, 1999). Since its conception, the program has brought university graduates from several English-speaking countries into Japanese classrooms as Assistant English Teachers (AETs), exposing Japanese students to English from a young age.

Though the government reported in 1996 that it saw an improvement in students' speaking ability (Moore \& Lamie, 1996), many AETs have reported that they were merely asked to demonstrate the correct pronunciation of a given word (Gilfert, 1999) and occasionally were asked to serve as consultants on language mechanics such as grammar, 
spelling, or punctuation (Moore \& Lamie, 1996). Due to the Ministry of Education's 1989 reform that was designed to encourage teachers to place more emphasis on listening and speaking in the ESL classroom, writing was not prioritized (Gilfert, 1999). However, MEXT's (the Ministry of Education became the Ministry of Education, Culture, Science and Sports known as MEXT in 2000) revised 2002 "Course of Study" proclaimed that communication skills now included writing (Matsubara, 2001) and encouraged AETs to modify their curricula to demonstrate a communicative approach to language teaching (LoCastro, 1996; Duppenthaler, Viswat, \& Onaka, 1989), a method unfamiliar to many of the Japanese teachers who were primarily responsible for leading the English classroom activities. However, this implementation may have been little more than superficial for some. In a 28 October 2010 Metropolis magazine article, journalist and former JET Lisa Gay reflects on her and her colleagues' experiences as a "human tape recorder" and admits that, "I was overpaid, underused and had little effect on my students' English skills" (para.3).

Though not directly stated, Inoue's 1997 studies suggest that students who do not receive formal English or Japanese writing instruction at any point during their pre-university years may be ill-equipped to enroll in university-level English composition classes both overseas and domestically due to their inability to differentiate between academic and colloquial writing styles and their tendency to imitate previously seen writing styles (Casanave, 2002). While many students attend preparatory juku (cram schools) or study English outside of their high school EFL classes, they generally learn little more than vocabulary and new sentence structures - the tools they will need to conquer and pass the "essay" section of the entrance exams that most universities added during the 1990s (Kajiki, 1996). These "essays" are typically 100-word, free-style compositions that are graded and marked based on grammatical accuracy rather than content-neither the ability to convey one's thoughts clearly nor the presence of writer's voice is assessed.

The lack of necessity for Japanese students to demonstrate a comprehensive grasp of writing is reflected in Japan's exam culture in which, ultimately, writing does not fit and is therefore often not included in exams. A major reason for this is that in exams, writing is regarded as too difficult to assess (Hamp-Lyons, 2007). The assessment of content in extended English composition in writing courses requires knowledge that assessors cannot 
access easily, due to limited language proficiency on the part of the teachers, and thus the skill of writing extended texts remains neglected in a culture that values examination scores over ability to communicate (Gilfert, 1999; Moore \& Lamie, 1996; Taylor \& Taylor, 1995).

\subsubsection{English L2 writing at the university level in Japan}

Loveday (1996) described a general failure of English language teaching in Japan as being due to a continuation of exam-based study in higher education. The problems include emphasis on grammar-translation, wash-back from entrance exams, and continued "reductionist concentration on receptive skills for decoding foreign texts" (Poole, 2005, p.244). This way of thinking and the limitation it presents to writing education goals has greatly hindered students and teachers at Japanese universities from understanding the processes writers go through in developing meaningful writing skills, both in building on students' knowledge of the L1 and using that knowledge to develop new strategies for writing in the L2. Matsuda (1997) explains that the problem lies with EFL teachers who do not incorporate revisions and discussions of revisions into the writing process. These teachers read their students' EFL writing without asking about their intentions, which does nothing to reveal the writers' thought patterns or "the rhetorical patterns of L1 written discourse" (Matsuda, 1997, p. 50).

It is important to note that while other countries have moved away from using grammartranslation methodologies in the ESL classroom after a shift in academic beliefs during the mid-to-late 20th century, the English language curriculum followed in many of Japan's schools still contains traces of direct translation teaching ideologies (Casanave, 2002). For many native Japanese teachers of English writing, the continued reliance on the grammartranslation method is explained by the role of culture, conceptions of the teacher's role, teachers' lack of training in other methodologies, as well as a necessity to practice grammar and translation as they are the skills required to pass examinations (Moore \& Lamie, 1996). In universities, the importance of passing examinations is less emphasized, and according to Casanave (2002) in her book Writing Games, this is where grammar-translation leads to the product approach. This shift in approach-though not a universal one-is related to the inclusion of genre theory and writing for specific purposes (Belcher, 2004). With the 
new focus on writing for specific purposes, genre studies (sometimes identified as English for Specific Purposes itself) led to a natural progression of the product approach.

As discussed earlier, process writing became a popular approach in teaching L2 writing in ESL settings in the 1980s, at the same time that communication skills became the focus for curricula and policy-makers. However, the process revolution may never have actually happened in Japan (Casanave, 2003). Further, it remains debatable whether process writing is actually more effective than product or form writing (Canagarajah, 2002). A Kyoto researcher and teacher, Yoshimura (2001), conducted an experimental study echoing the work of two Japan-based researchers, Oi and Kamimura (1997), who found success for Japanese university students in learning English language argumentative essay patterns as well as organizational patterns and coherence structure. Yoshimura acknowledges criticisms of teaching the form as opposed to the process and content of essay writing, but is convinced that the benefit for beginning Japanese writing students comes when they are comfortable with a form of writing. Yoshimura goes on to explain that their success with form can then be transferred to future writing contexts (Connor, 2003). Both Yoshimura and Matsuda (2001), suggest Japanese students are more comfortable with a form of writing because of a lack of familiarity with English writing strategies. Anthony (2000) refers to the teaching of form as the teaching of a genre. In a study conducted by Anthony (2000) in a university in Japan, it was noted that students with considerable knowledge in their subject areas could develop non-formal aspects in written text. He maintains however, that

if the learners had little experience with the target context, a course aimed at developing writing skills needed to operate in that context would be destined to fail. In such cases, a focus on the more easily observable, formal features of the target genre would perhaps be more effective (p.18).

Because English is a foreign language in Japan and not a second language, students do not recognize the value of English in their everyday lives. Also the time frame for a foreign language course tends to be short in Japanese universities-usually fifteen once-a-week 90-minute lessons over a period of one semester (Anthony, 2000). Although approaches in process writing pedagogy may be useful, Anthony (2000, p.18) explains that 
most have been developed in classrooms where the learners are either native speakers or approaching native speaker levels. In a foreign language classroom, on the other hand, few learners will be at an advanced level, and many will be struggling with even basic vocabulary and grammar points. To ask such learners to analyse texts and negotiate the writer's purpose, audience's assumptions, and so on is clearly unrealistic.

Based on the observations of Anthony (2000), it seems that the development of process writing did not necessarily have a place in Japanese universities due to a general lack of proficiency of the students. Therefore, instead of taking up major changes in pedagogy as part of the process revolution, teachers of English writing in Japan have instead maintained grammar-translation methodology. Sensing that L2 writing pedagogy was in need of some innovation without taking up the apparently "unrealistic" efforts of process writing, scholars and researchers of the teaching of English writing in Japan have been looking more towards social and political aspects instead of linguistic and textual aspects in finished written products. Casanave (2003), in her discussion of the debate over Japan's position on the process and "post-process" movements, explains that a socio-political perspective needs to be expanded in order to explore the diversity of individual writers and writing contexts, echoing the suggestions of other researchers (Olson \& Dobrin, 1994; Dobrin, 1997 in Heard, 2008). This emphasis is centered on the understanding that L2 writing education in Japan was not influenced by the process movement, due to cultural, social and political constraints on the writing classroom that are not conducive to process writing.

\subsubsection{Application and debate of process writing in university English L2 writing in Japan}

Although it has been argued that the process revolution never came to Japan, some teachers of English writing in Japan did adopt certain aspects of the writing process that provided an introduction to students of the basics of pre-writing, drafting and revising, with a particular focus on peer reading. However, Muncie (2000) suggests that process writing in Japan is ineffective in that students have no choice in using feedback from their teachers who are considered experts-perhaps a remnant of the traditional Confucian master-pupil relationship discussed earlier in this section. Students, therefore, are in no position to negotiate with redrafts; instead they must follow their expert teacher's advice 
as closely as possible. Muncie argues that the feedback therefore loses any value it may have had due to a lack of meaningful student involvement.

However, some studies have shown benefits of feedback in EFL writing contexts in Japan. In a trial with university students in Japan who were taking an academic English writing course, the use of peer feedback on mid-drafts and teacher feedback on final drafts encouraged writer autonomy (Muncie, 2000). The students were required to produce a summary using points from both peer and teacher feedback, which allowed the feedback to have more long-term effect on students' future writing. LoCastro (2000), in a study on peer feedback on English academic writing by first year students in a competitive Japanese university (TOEFL scores ranged from 500 to 590), found that even over a relatively short amount of time practicing peer reading, students were able to provide more corrections and less 'I think' expressions (with the understanding that the use of 'I think' expressions suggests a lack of evidential support, or the understanding that it is generally an unnecessary expression). This further suggests that instruction in peer reading leads to greater ability to function critically in the EFL writing classroom.

Though English L2 teaching methodologies in English-medium contexts have undergone drastic changes, they are now predominantly based on genre-based writing instruction and post-process approach and theory (Hyland, 2007). In Japan, the effectiveness of process writing continues to be debated. While Gilfert (1999) insists that university writing teachers in Japan need to use the process approach, both Anthony (2000) and Muncie (2000) argue that the process approach is ineffective in Japan. In his essay, Matsubara (2001), a Japanese teacher of English, made a plea for other English teachers in Japan both native and non-native to integrate situational writing (i.e. writing meant to prepare students for real-life situations) into their teaching, in response to teachers having not actively employed anything more than the outdated grammar-translation method. This lack of active compliance can be attributed to teachers' lack of training in other English L2 teaching methodologies and the pressure put on both educators and students to nurture children who will be able to pass not only university entrance exams but also the Test of English for International Communication, or TOEIC, which many universities in Japan take into account (Chapman, 2004). 
Many acknowledge the idea that teacher-student negotiation and teacher and peer feedback is important to both the writing process (Kamimura, 2006) and the cultivation of a student's writing ability and voice (Helms-Park \& Stapleton, 2003; Matsuda \& Tardy, 2007; Stapleton \& Helms-Park, 2008), even though Japan has not fully integrated postprocess writing into the university-level ESL curriculum (Casanave, 2003). However, though essays are often assigned by university teachers in Japan to judge achievement and performance (Connor, 2003), the feedback received on these assignments is often limited. This lack of feedback may be the result of teachers' assessment of essays as a form of examination due to an educational culture that is more concerned with comprehension and direct translation skills (Moore \& Lamie, 1996).

Casanave (2003) goes so far as to suggest that ongoing feedback between teachers and students in Japan is often inadequate and sometimes completely non-existent. In a report of her findings, she remarks that it was common for students in Japan to submit written assignments and to receive a mark without seeing the assignment again or being provided with any verbal or written feedback-a tendency that reflects the Confucian ideal that a student should not question his or her instructor or teacher (Stapleton, 1995).

This finding reflects that of Gorsuch (1999), who examined the attitudes of Japanese teachers of English toward communicative language teaching in their approach to English language teaching. She found that Japanese teachers approved of highly controlled activities that did not ask students to produce extemporaneous compositions. The contrast between the approach of Japanese teachers of English and the typical approach of native English-speaking teachers of English is highlighted in Poole's (2005) observation that Japanese and native-English teachers were segregated, due mostly to a perceived gap in teaching methodology. The native-English teachers saw the Japanese teachers of English as stuck in grammar-translation while the Japanese teachers saw the native-English teachers as lacking seriousness. For unmotivated students, this conflict between teachers meant that systems were in place for them to pass their English classes easily without doing much work, in order to avoid further conflict. 


\subsection{Limitations of previous research}

Most of the studies concerning the English writing skills of Japanese students have been retrospective accounts of students' experiences rather than any actual examination of their experiences in situ, or they have focused on the analysis of English language written composition of Japanese high school students (Inoue, 1997; McFreely, 1999) and university students (Spack; 1997; Yasuda, 2005) outside Japan. There have been a number of studies-often smaller studies conducted by the same researchers and academics including Hirose (1998; 2001; 2003; 2005), Stapleton (2001; 2002a; 2002b), Helms-Park \& Stapleton (2003), Kamimura (1996; 2000), and Kamimura \& Oi (1997; 1998; 2001; 2006)—that have focused on the experiences of students learning to write critically in English at a university in Japan, but these have been focused on shorter periods of time, often analysing only the results of a single writing task.

Casanave's (2002) book Writing Games provided a rich description and analysis of writing "game strategies" practised by Japanese writers of English at the undergraduate and postgraduate levels with particular focus on the writing teachers, rather than an in-depth analysis of the students' experiences. The recent ethnographic study by Poole (2010) examined the actual writing interactions, negotiations and activities of students in a Japanese university. Poole's study, published as a book entitled The Japanese Professor: An Ethnography of a University Faculty uses a very similar methodology to mine in the use of observations, informal interviews and an analysis of students' written texts. However, the focus of Poole's study was on the functionality of the Japanese university system as a whole rather than the experiences of the students learning written critical argument in English within that system.

This study aims at exploring the experiences of both teachers and students of the teaching and learning of writing in English in a Japanese tertiary institution. The study maintains a focus on the continually reported lack of success of EFL education in Japan. It examines approaches to the teaching of writing and what considerations Japanese and native university teachers of English are making. Key issues regarding students' and teachers' cultural understanding of English were clearly outlined by Fox (1994). Fox's study raised a 
crucial question that is a key consideration for the outcome of my study: Which needs to change their perspective, the university or the student?

\subsection{The growing concern for university-level writing education}

The recently published results from the Collegiate Learning Assessment (CLA) Longitudinal Project in the book Academically Adrift: Limited Learning on College Campuses (Arum \& Roksa, 2011), have raised concerns about the integrity and value of higher education in the US, particularly regarding writing and reasoning skills. The project was conducted with 2,362 students at 22 different four-year institutions in the US from 2005-2009. The book starts with a distressing quote from former Harvard University president Derek Bok:

Colleges and universities, for all the benefits they bring, accomplish far less for their students than they should. [Today's students graduate] without being able to write well enough to satisfy their employers... [or] reason clearly... (Bok 2006, p.8 cited in Arum \& Roksa, 2011, p.1)

These concerns have been increasingly shared by university presidents, policymakers, and stakeholders. The issues raised by Arum and Roksa point to two central factors: students' "contempt" for or disinterest in academic learning (p.82) and desire to spend more time on social and leisure activities (p.59), and professors' lack of rigor in their course requirements (p.31). It has been confirmed by others such as Mack-Cozzo (2002) (discussed in section 2.2.2), that university students in Japan share a similar disinterest for academic learning. She explains, "Their disengagement from learning is so fundamental that they often pack themselves in the farthest back seats of the classroom, so they can gossip, sleep, or use cell phones undisturbed" (para.12). They also maintain a much more social-heavy use of time, dedicating only a small portion of their time to academic achievements (Mack-Cozzo, 2002).

As for professors' lack of rigor, this is a more difficult factor with which to draw a comparison. An important aspect of this issue is what Goodman (2005) describes as the "academic freedom" university teachers in Japan are given, in which courses and assessments are made by individual teachers with no external evaluation. This freedom has caused disillusionment in some students who choose not to attend classes, yet they still pass because of the teachers' sense of obligation to the university for accepting them. To 
suggest that a lack of rigor is caused only by professors' cynicism or laziness would be both unfair and wrong, although Mack-Cozzo (2002) describes her experience with typical Japanese professors very negatively. In her experience, Japanese professors seemed to be very lazy:

Most Japanese sensei tolerate such [poor student] behavior, sitting at the desk in the front of the classroom and burying their heads in the textbook from which they read. They rarely get up, even to write on the blackboard. They are poorly prepared for their classes and rarely comment on student papers. (p.47)

While to a certain degree these problems may be present both in the US and Japan, in Japanese higher education the expectations for rigorous coursework are traditionally different. Demanding course requirements are only possible in situations where students are able to meet those demands. Because the students in Japan take so many courses in their first two years, there is less time outside class to do homework. Arum and Roksa suggest courses that require at least 40 pages of reading per week and at least 20 pages of writing per semester ultimately lead to improved results in writing and reasoning ability, although this is referring to mostly native English-speaking students and not specifically regarding writing courses. University teachers in Japan know there is a limit to how much work can be assigned outside class, and they also understand language proficiency limitations on reading assignments, so students in Japan cannot be assigned as much homework, particularly tasks in a foreign language.

Opportunities for learning to think critically and communicate effectively are limited for university students in the US according to Arum and Roksa (p.35). The situation in Japanese higher education regarding critical thinking and effective communication (discussed in more detail in chapter 3 ) seems potentially more dire as it is less consistent in an ongoing state of flux. Although university students in the US may be deprived of developing critical thinking skills due to a lack of reading and writing tasks, they are not deliberately denied or steered away from critical thinking as suggested occurs in Japanese universities (e.g. Davidson, 1995; Mack-Cozzo, 2002). 


\subsection{Conclusion}

Aspinall's (2003) five major reasons why EFL education in Japan has been unsuccessful offered an important line of focus for my study in that it was necessary to consider contrastive rhetoric, and to challenge generalizations made about Japan's language education culture. While reasons 1-4 have been addressed, and in some cases, disproven, Aspinall's fifth point requires more investigation of the extent popular culture has impacted on Japanese individuals' desire to truly learn the English language and to understand its culture beyond just basic eikaiwa (English conversation) skills. Also, it needs to be discerned whether the likability of one's teacher and interest in and familiarity with topics used in class actively impacts on students' approaches to critical thinking in the generation of arguments and motivation to learn.

It can be argued that Japanese university students studying EFL must learn to demonstrate and use their critical thinking abilities in order to write coherently and cohesively. However, effecting a drastic change to the EFL curriculum in Japan could take years (Poole, 2005) due to the structure and corporate nature of the Japanese university system. Furthermore, these changes not only have structural implications but also cultural ones. The elements of aimai and haragei may lead Western teachers and researchers to interpret Japanese students as having inhibited ability to develop an individual sense of voice and personality in their writing. While the teaching methodologies in many Japanese EFL classrooms have indeed changed over the most recent decades, it is still necessary for both native and non-native instructors at the university level to have a basic understanding of the fundamentals that fuel Japanese culture (Stapleton, 2001) in order to teach their students more effectively.

Based on this review of relevant literature, there is a clear gap in the research that investigates current practices of English writing education in Japan, in terms of teaching practices and analysis of student writing output. This gap is what led me to conduct my study with Japanese university students in their English language writing classes in Japan in order to gain insight on what actually happens in these classes. As this issue is too broad for one study to address, I have chosen to focus on the specific aspect of writing education that is central to much of the criticism of Japanese student writing-that of critical 
thinking, or a lack thereof, in Japanese students' writing. In my study I have chosen to investigate critical thinking within the theoretical parameters of critical argumentation and writer identity-the importance of which will be discussed in the following chapter outlining the key theoretical frameworks of this study. 


\section{Chapter 3. Social constructivism: The theoretical framework}

This chapter provides an overview of the theoretical framework of the study. It will begin by connecting the background on EFL education research in Japan, which was presented in the previous chapter, to the theories behind the study. This connection highlights the reason critical argument and writer identity are significant areas of focus for the study. The chapter then moves into a deeper discussion of social constructivism as the fundamental philosophy behind the study, including an examination of some of the criticisms against it. After that, a description of how constructivist theory is applied in education is provided. Based on these theoretical underpinnings and the context of the study described in chapter 1, a diagrammatic representation of the theoretical framework and a detailed explanation of it are then provided. With consideration to the connections between the significant theories of the study, a discussion of the phenomena of developing cultural and academic identities and developing critical argument is then explored further, leading to a focus on critical writing as mediated action. The chapter concludes with a summary highlighting the contributions of each significant theory to the theoretical framework of the study.

\subsection{Why focus on critical argument and writer identity?}

In the previous chapter, it was emphasized that research in EFL education in Japan has revealed writing to be the most problematic skill area for students. It has been described as "neglected" (Davies, 1999) and the least competent skill of English of university students of EFL in Japan (Kroll, 1990), particularly with regard to applying critical thinking skills to developing critical argument (Kamimura \& Oi, 2006; Rabbini, 2003; Stapleton 2001; 2002a) and establishing writer identity (Casanave, 2002; Matsuda, 2001), both central elements of "socio-cultural theory" (Lantolf \& Thorne, 2006) as it applies to academic writing education research. As a comprehensive theory of writing has yet to be established (Sasaki, 2005), writing education is shaped by the environment in which it is developed. Taking into consideration the social and cultural aspects of the environment, English writing education in Japan is often reduced to grammatical and lexical studies for the purposes of examinations, since there is not much further need for English writing ability beyond this level (Rabbini, 2003). 
However, this level of writing education offers very little in terms of sustainability of English language skills and their practical application; it does not consider the development of thinking skills or strategies for creating logical relationships between thoughts (Shinoda, 2006). Critical arguments are often not required and therefore not developed, and no real consideration is normally given to issues surrounding writer identity (Stapleton, 2002b). In order to introduce more of a focus on critical writing, some teachers and curriculum developers are taking social constructivist positions in continuing to look toward developing students' socio-cultural awareness in EFL writing in Japan in an attempt to better connect the social and cultural relationships of students with their English writing (Rabbini, 2003; Rinnert \& Kobayashi, 2001).

It is important to point out that much of the emphasis on developing students' sociocultural awareness focuses on their development as critical thinkers. In the 1980s, as social constructivist theories were gaining credibility internationally, the Ministry of Education of Japan made steps to move away from exam-based English education, as it was not preparing Japanese graduates for successful competition in international business. More emphasis was put on kokusaika, or 'internationalization' (Kubota, 2002). The focus was more on practical communicative skills. In another move around the same time, in response to increased examination-related stress issues, the Ministry introduced yutori kyoiku, or 'relaxed education' (Butler, 2007). It was a challenge to make these changes at the same time, as the new style of education involved a major ideological shift, putting more emphasis on building socio-cultural awareness through internationalization, individuality, sustainable lifelong learning, and adjustment to social change.

This ideological shift led to teachers giving increased consideration to sociological factors. Each student brings his or her own social and cultural identity to the language classroom, often in great contrast to that of the teacher. The factors that affect people's socio-cultural identities are based on the classroom itself, the interpersonal contexts in the classroom, their purposes for being there, and their personal backgrounds (Duff \& Uchida, 1997). These identities evolve in the classroom. Within its own social and cultural situation, a student's socio-cultural positionality in the classroom impacts heavily on motivations for learning. This positionality is the student's sense of self, and the social relations that are 
affected by this sense (Anthias, 2002). Vygotsky's (1978) concept of collaborative learning - that all learning, even learning to think, starts with interaction-leads students to create knowledge through their social relations and interactions. Wenger (1998) suggests the negotiation in these interactions is how students develop new identities in language learning. As writing is a communicative act, situated in a social, cultural setting (Casanave, 2003; Rinnert \& Kobayashi, 2001), it is necessary for a student to establish an awareness of his or her own socio-cultural positionality in relation to others to be able to develop writing skills. This concept-students' establishment of an awareness of his or her own socio-cultural positionality-is the basis for the particular focus of this study on students' establishing writer identity and developing critical argument.

\subsection{Philosophy behind the study: Why social constructivism?}

The central philosophy behind the study is social constructivism-introduced in chapter one as a learning theory based on the ideas of Vygotsky (1978) that human development is socially situated and knowledge is constructed through interaction with others. Creswell (2009) asserts that social constructivism serves as a useful theoretical framework as it allows for necessary qualitative analysis to reveal insights on how people interact with the world. Social constructivist theory asserts that people's ideas coincide with their experiences. With this understanding, writers build on their socio-cultural awareness, a key point in identity construction. This section will first provide brief background information on social constructivism in academic writing research, followed by an overview of studies in L2 writing that have utilized social constructivism as a base theory. I will then look at some current studies in L2 writing and explain how social constructivism was applied in these studies in order to describe the implications such approaches to social constructivism had on my study. I will conclude this section by explaining the constructs I am drawing on from these studies.

\subsubsection{Social constructivism in education and identity construction}

In a constructivist approach, the generally agreed concept is one that was developed in the early twentieth century with a focus on children's education, that in collective education, learning is simultaneously an individual and a social process (Simon, 2010). The concept 
was developed separately by psychologists Jean Piaget and Lev Vygotsky. In the late 1920s, Piaget's "theory of cognitive development" asserted that knowledge is constructed from active participation in the learning community. At around the same time, Vygotsky developed the constructivist concept through his establishment of cultural-historical psychology, though this was relatively unknown in the West at that time. These two theories both focused on the fundamental role of social interaction in the process of a child's cognitive development. An important difference, however, is that Piaget's understanding is of cognitive constructivism, where a child's development precedes social learning, whereas Vygotsky's understanding is of social constructivism, in which social learning precedes development. A widely quoted statement from Vygotsky reads: "Every function in the child's cultural development appears twice: first, on the social level, and later, on the individual level; first, between people ... and then inside the child" (Vygotsky, 1978, p. 57). However, while Piaget's theory heavily influenced Western education at the time, it was not until the 1960s, decades after his death, that Vygotsky's theories came to have a heavy influence on Western classroom practices. $^{2}$

In the 1960s-70s in the West there were significant developments of social constructivist theory that focused on children's education. These developments included valuable contributions from American psychologist Jerome Bruner as well as posthumous translations of works by Vygotsky. Bruner's The Process of Education: Towards a theory of instruction (1966) proposed integrated representations (action-, image- and languagebased) of children's cognitive development. This was significant in that it stood in contrast to Piaget who had proposed that such representations would be achieved in stages. Bruner insisted that any learner should be capable of learning if instruction is appropriately organized and builds on previous learning. Bruner's theory had a great impact on education in the West at the time and supported Vygotsky's theories, which were finally

\footnotetext{
${ }^{2}$ An important distinction with respect to Vygotsky's development of constructivist theory in education is that there are two versions. One is "socio-interactional" and blends Piaget's theory of cognitive development and Vygotsky's cultural-historical psychology. The other was founded by Vygotsky and expanded in his notion of activity theory (Vianna \& Stetsenko 2006). The constructivist theory this study utilizes is sociointeractional.
} 
being introduced to the West through translated publications. Perhaps most significant, Vygotsky's Mind in Society: The Development of Higher Psychological Processes (1978) introduced the notion of the Zone of Proximal Development (ZPD), used to show the discrepancy between how much a learner can do with helpful interaction from others with experience and knowledge, in contrast to what a learner can do independently. This aspect of constructivist theory led to important changes in children's education.

Although constructivist theory is most often applied to children's education, in higher education it is especially effective in academic writing research as a solution to the "resistance to learning" that adult learners typically experience (Atherton, 2011). Because constructivist learning requires an explanation of why it is important to learn something, and is guiding, not leading, the student to her/his own understanding of the subject matter, it provides necessary motivation in higher education. It helps adult learners to overcome the resistance to learning by making associations to a learner's past experience. This motivates learners to move past existing ideas about learning as well as any inhibiting behaviors.

For adult learners, constructivist learning has significant implications in that, with many years of experience and accumulated schemata, new information must be connected to neurological structures already in existence. Cultural identity building is an ongoing process that is affected by various social, cultural and historical factors that are especially realized in education settings, as illustrated by Gomez-Estern, et al. (2010):

[C]ultural identity is not an immutable "essence" placed in the heart of our mind, but a malleable process that is constructed in relation to the social settings (such as formal education) in which people participate. (p.231)

The fact that the process in the construction of cultural identity is participatory allows learners to take ownership of that construction.

In their examination of social constructivist theory, Stetsenko and Arievitch (1997) argue, "constructing the self and deconstructing it can be viewed as the two alternative strategies ensuing from the socioconstructivist approach" (p.159). They offer an analysis of the two divergent theoretical/methodological perspectives within the social constructivist 
framework-the first being discourse-based, which sees the self in the social reality of discourse; and the second a post-Vygotskian perspective, which sees a guided formation of the self. These two perspectives do not view an individual as self-contained, but rather offer "a relational, contextualized account of the evolving self" (p.159).

Stetsenko and Arievitch (1997) go on to explain that the discourse-based approach is insufficient for identity construction in that it separates the self from its psychological processes. They assert that, like the post-Vygotskian approach, the central focus in discourse-based approaches is the idea that "language use is taken as a root metaphor for all human action, and conversation, dialogue, as the root model for the analysis of all mental processes" (p.162). Where the discourse-based approach diverges is in its methodological application, in which the positivist methods of a naturalist inquiry are altered in a deconstruction of the self, designed to reveal the social-cultural nature of phenomena. In contrast, the post-Vygotskian perspective focuses on the positivist methods of active co-construction of the self, a key concept in my study.

\subsubsection{Social constructivism in academic writing research}

Social constructivism in academic writing research developed in response to, and as a critique of, expressivist theory and practice that had been popularized by composition researcher Peter Elbow. The expressivist construct encouraged autobiography and writing about personal experiences, which had developed with the increased attention on learners' social and cultural history. The emerging social constructivist theory emphasized the self and subjectivity as textual constructions, not natural attributes (Cain, 1995).

Kenneth Bruffee is attributed as popularizing the social constructivist construct in the 1980s. Although Bruffee (1986) described social constructivism in classroom practices

with its emphasis on collaborative learning as at first resembling both the methods and principles of expressivism as it was presented by Elbow in his book Writing Without Teacher (1973), social constructivism stood in opposition to expressivism. The argument was that expressivism reinforced institutional and cultural norms rather than challenging them, as constructivism promoted. The reinforcement came in the form of establishing an expressivist, autonomous self that maintained social inequalities. Cain (1995, p.25) states, 
"social constructivists argued against the cultural limitations of expressivist approaches and instead focused on the ways power and authority shape what teachers, students, and writers think and write". Because of this, social constructivism in academic writing research put more focus on the social contexts of learning, which led to more consideration being given to identity construction.

With a focus on social context and identity construction, academic writing research sought ways to move beyond expressivism, which was viewed as focusing on the self through emphasis on internal beliefs and nature, and into the more social constructivist philosophical concept of "externalism". In composition studies externalism sees the written product as a result of not only what the writer has processed internally but also the social and cultural/historical/political context in which the piece is created (Russell, 1993). In order to go beyond the entrenched dichotomy, Bawarshi (1997) proposed a means of mediation between the social constructivist (or contextual/institutional) and expressivist (or personal) positions. Bawarshi proposed a need for a new theory, one of an expanded social constructivist nature, to bring together the autonomous self and the constituted self in composition studies-something this study has aimed to explore.

Ravelli and Ellis (2005) in their volume Analysing Academic Writing: Contextualized Framework, state that by the early 2000s, academic writing research had come to highlight "some of the ways in which students negotiate identity, construct roles and develop argumentative positions, engage in technologically supported writing processes, and deal with the demands of specialized disciplines and of a language that may not be their own" (p.1). In the same volume, Hyland (2005) explains that academic writing research moved beyond ideational analysis of students' written texts in order to explore the interpersonal function of those texts. This movement promoted the idea that students' written texts do not just "represent an external reality, but use language to acknowledge, construct, and negotiate social relations" (Hyland, 2005, p.5). Success in academic writing came to be based on the writer's ability to establish a clear voice in relation to the reader, and to evaluate alternative viewpoints. The concern with the interpersonal reflected the development of both social constructivist as well as systemic functional frameworks such 
as Appraisal (Martin, 2000), the main framework utilized in the analysis of students' written texts in this study.

\subsubsection{An overview of social constructivist research on L2 writing}

Publications that included social constructivist research in L2 writing first appeared in the 1990s as the academic field of L2 writing made its emergence in publications that included "second language writing" in their title (Matsuda, 2003). At that time L2 writing was "com[ing] into its own as a field of inquiry, generating diverse research and scholarship" (Hedgcock, 2005, p.597). A specific focus on social constructivist research in L2 writing was addressed in Santos' (1992) article in the inaugural issue of the Journal of Second Language Writing. As mentioned in the previous chapter, Santos (1992) described L1 writing education as ideological and L2 writing education as pragmatic. This description was offered in an analysis of the differences in social constructivist approaches in L1 and L2 writing. Santos claimed L1 composition to be ideological because of its focus on power and politics. Social constructivism in L1 writing emphasized students' critical thinking in the writing process by challenging those power structures that had an influence on their lives. In contrast, social constructivism in L2 writing was more pragmatic in that it steered students toward meeting the immediate needs, such as understanding task assignments and structuring appropriate essays, involved in completing their academic writing tasks (Costino \& Hyon, 2011). Santos made the statement that "pursuing political goals and/or changing students' sociopolitical consciousness is not on the ESL writing agenda" (1992, p.9). She further emphasized the L2 writing context as one in which instructors are not meant to change their students' value structures.

Another early publication on social constructivism in L2 writing research by Carson and Nelson (1994) focused on the social constructivist distinction between group learning and collaborative learning as it was described by Bruffee (1986). Group learning involves students working together for individual benefit, while collaborative learning involves students working together for mutual benefit. Student to student collaboration in L2 writing through such practices as peer reading and peer feedback was being encouraged in the 1980s. However, these practices were applied with some hesitation as they came 
directly from L1 writing. Both Santos (1992) and Silva (1993) suggested that adopting L1 writing practices into L2 needed to be done critically, and adapted appropriately.

Notions of conformity in group learning have significant implications for studies in EFL education in Japan, since conformity seems to fit into historical descriptions of Japanese collective ways of thinking, as addressed in the previous chapter. Carson and Nelson (1994) looked at earlier studies with Japanese students outside Japan (see Frager, 1970; Nakane, 1970; Mann, 1980) that revealed surprisingly lower rates of conformity among these students-a suggested reason being that conformity was not desired in newly formed groups, such as Western academic communities. This has direct implications for this study in that in writer identity construction, Japanese students may be consciously refusing to conform to the constraints of a Western academic community such as the one found in an academic English writing classroom. That it is a foreign academic community may be the trigger for the students' refusal to conform.

Social constructivism and its emphasis on students' socio-cultural positions, was a decidedly Western approach and therefore first applied to ESL research, rather than EFL. Santos (1992) pointed out that the EFL distinction is an important one because the frame of reference in early studies (see e.g. Bizzell, 1978; 1982) focused on American society in the ESL context. However, English L2 critical pedagogy faces different challenges in nonEnglish speaking countries where classroom ideologies may stand in contrast to American ones. Ramanathan and Atkinson (1999) suggested that the application of a social constructivist approach to L2 writing research was part of the movement of critiques of written voice. Since my study takes place in an EFL context with a focus on students' establishment of voice in critical writing, conducting research within a social constructivist framework contributes to this movement.

Ramanathan and Atkinson (1999, p.52-53) reported on Scollon's 1991 study where he attempted to make use of Elbow's North American-based process approach in an EFL writing class in Taiwan, and found that the use of voice was different from the personal expressivism described by Elbow. Scollon described Western student writers as "ready to adopt" a rhetorical situation in which they are not affected by the social context. Instead 
Western students are able to express themselves without those external pressures. In contrast, Scollon found the Taiwanese students were less concerned with expressing themselves than they were with achieving integration with the scholarly community. This is strikingly different from Carson and Nelson's (1994) findings from earlier studies where Japanese students were less likely to conform to the academic community.

In a special issue of the Journal of Second Language Writing that focused on voice of the L2 writer, Atkinson (2001) expanded on his own L2 writing research as well as those of others in the same issue (Matsuda, 2001-as mentioned in previous chapter; Prior, 2001; Ivanič \& Camps, 2001-as mentioned in previous chapter; and Hirvela \& Belcher 2001). Atkinson asserts in his reflections on these articles that voice construction is complex for any writer. He notes that social constructivism provided "modern versions" of the voice concept in these more sophisticated perspectives of voice that were not used to "refurbish" the individualist voice that had been the focus of L2 writing research in the 1990s. Rather, they served as valuable departure points from expressivist process-oriented composition studies. By the early 2000s, L2 writing research paid more attention to social constructivist process-oriented notions in composition, such as Vygotsky's concept of scaffolding.

The Vygotsky-inspired social constructivist focus on L2 writing came in the social interaction of guided development in the form of scaffolding, as described by Donato (1994) focusing on speaking skills, and expanded by Hyland (2003) focusing on L2 writing skills. Scaffolding maintains the social constructivist view that through interaction with a more experienced or knowledgeable other person, a learner can participate in the development of her/his own skills to reach higher competency (Donato, 1994). Hyland (2003) explains in teaching composition, scaffolding comes in the form of heavily assisted instruction in the early stages, with extensive modeling of the writing process. The heavy support in the early stages is then relaxed in order to encourage student writers to actively participate through negotiation of the writing process through peer and tutor feedback, making them more independent, autonomous writers, eventually able to construct pieces on their own. 
Vygotsky's theories, including scaffolding, have been criticized in that his explanation of development rests on mediation and externalization, as well as internalization (Daniels, 2006). The concept of 'mediation' allows for the development of a more liberal understanding in which mediators (interventions) function as situations in which a learner becomes aware of her/his social, cultural and historical influences, takes action on them and is acted upon by them during the learning process. In the field of psychology, competing definitions of 'culture' (normally labeled socio-cultural or cultural-historical) create debate about the nature of these factors (Daniels, 2006). However, in second language acquisition research, the use of the term "socio-cultural theory" has been applied as a necessary umbrella term for the multiple lineages of Vygotsky-inspired research (Lantolf \& Thorne 2006, p.3), and therefore serves as a clear link between sociointeractional constructivist theory and the features of critical argument and writer identity in Japanese university academic EFL writing selected as the focus for this study.

\subsubsection{Social constructivist approaches in current L2 writing research}

With the constructivist focus on the social context and larger community of learners, there has been a major shift away from individually-based L2 writing instruction to instruction that sees knowledge-building as a co-constructed process. The greatest contribution of social constructivism to education may be the socio-cultural theoretical shift in emphasis from knowledge as a product, to knowing as a process. The current social constructivism approaches in L2 writing research range in their specific areas of focus, with most publications discussing epistemological issues related to social constructivism in educational fields including mathematics, science, and social studies, and very few reporting results of studies utilizing social constructivism as a theoretical framework in L2 writing. This section will provide a brief overview of four recent studies showing a range of applications of social constructivism, including scaffolding (Cotterall \& Cohen, 2003), collaborative writing (Storch, 2005), identity construction (Abasi, Akbari \& Graves, 2006), and intercultural learning experiences (Hung \& Hyun, 2010).

Cotterall and Cohen's (2003) study took a distinctly social constructivist approach in its focus on scaffolding in English L2 writing at a university in New Zealand. In their discussion 
of related research, they cite Greene and Ackerman's (1995) article Expanding the constructivist metaphor to describe learners' understanding of their own position in relation to others. Their study followed 16 international students enrolled in a 12-week EAP writing program in which the students were taught academic writing skills through scaffolding in order to produce a 1000-word argument essay in English under examination conditions. They found that the scaffolding approach was highly effective. Students were able to understand the appropriate use of language and structure. In addition, because of the scaffolding they were able to establish the rhetorical context, not just the decontextualized mechanics of writing. With a clear sense of their writing purpose, the students were able to take ownership of their writing.

Storch's (2005) Collaborative writing: Product, process, and students' reflections reported on an Australian university classroom-based ESL study that examined students producing jointly written texts. She took a social constructivist position in her conceptualization of the study in that the focus was on the encouragement of student interaction and coconstruction of knowledge. The idea is related to Vygotsky's (1978) emphasis on the novice learner gaining knowledge from an expert, as a type of knowledge scaffolding. Storch insisted that this scaffolding does not occur only between student and teacher, but that it also occurs between peers in group or pair work, thus further supporting social constructivist theory, in this case, in the L2 writing classroom.

In the study, Storch attempted to move beyond the normally limited group or pair work activities that happen in the writing classroom such as peer reviewing in order to put more focus on the writing process rather than the product. She not only collected the students' written texts for analysis but also recorded dialogues between pairs in the collaboration process of producing the texts. She used quantitative analysis to focus on the writers' fluency, accuracy and complexity, and qualitative analysis to focus on content and structure. Though the quantitative analysis proved to be less significant, the qualitative analysis suggested that students managed to complete the writing task more effectively and successfully when writing collaboratively, offering opportunities to the students "to give and receive immediate feedback on language" (Storch, 2005, p. 168). 
Abasi, Akbari and Graves' (2006) study of Canadian graduate-level ESL students' identity construction strategies, though not specifically mentioning 'social constructivist theory', was clearly grounded in social constructivism. The study "adopt[ed] a sociocultural view on academic literacy and a closely related perspective on writing and identity... Based on this view, individuals read and write in ways specific to particular social groups" (p.104). Abasi et al used a social constructivist qualitative approach with students' written texts and interviews as data collected for their inquiry into students' awareness of identity construction and found that "students' awareness of the intersection of writing and identity closely matche[d] their socialization into their prospective disciplines" (p.113). They noted, 'students' prior socialization ha[d] a major role in their conception of the nature of knowledge and their relation to it" (p.114).

Most recently, Hung and Hyun (2010) used integrated principles of social constructivism to form the theoretical framework for their study on East Asian international graduate students' epistemological experiences in an American University. The study used interviews with seven East Asian PhD students in the US to explore their perspectives on their intercultural learning experiences. The focus was on how American universities could create more attractive prospects in their attempts to increase the number of international postgraduate students-an issue that arose as there were increasing negative experiences (such as culture shock and "study shock" caused by the unexpected differences in disciplinary approaches) reported by non-native English speaking international postgraduate students in the US. Through an in depth analysis of those interviews, the researchers found that there was great need for establishing a better academic support system that fosters increased intercultural awareness for both students and faculty.

\subsubsection{Constructs my study draws on}

My study draws on constructs from all of the recent studies described in the previous section. I will first outline the constructs as perspectives of social constructivism from each study before providing an example of the application of social constructivist theory to my data analysis plan. This is followed by a final statement of justification for using social constructivism in the approach to this study. 
Through a social constructivist approach, Cotterall and Cohen's (2003) ability to reveal that students were able to take ownership of their writing and establish an appropriate rhetorical context is significant. The constructs of writer ownership and establishing rhetorical context are both drawn on in my study. Storch's (2005) study showed that social constructivist theory is crucial to gaining a better understanding of the collaborative stages of the writing process. My study drew on the construct of co-construction of knowledge through working closely with peers and tutors. Abasi, et al. (2006) showed that social constructivism is integral in examining students' awareness of identity construction in their own writing. Accordingly, exploring students' awareness of their own writer identity is a central construct in my study. Finally, Hung \& Hyun (2010) particularly emphasized the importance of conducting research based in social constructivist theory, and even recommend that further research pursuing issues in this area focus on students' writing. The issue of universities generating more global appeal through a focus on intercultural awareness in L2 writing research is a crucial point in justifying the significance of utilizing a social constructivist approach in my study at this time.

To illustrate the social constructivist perspective as it was utilized in this study, I provide this example: In the case of one of the student participants, Aya from the D class, I observed in the class in which she presented her research that her classmates appeared receptive and able to provide what I had assessed as useful feedback. Later in my interview with Aya, she commented that she thought the feedback was probably useful but that she did not really understand it or know how to use it. In my interview with her teacher, he commented that the feedback was not particularly useful. These three different perspectives on the same student in-class feedback, from a constructivist point of view, are all accepted as valid meaning construction. Acting as observers, each of us made evaluations based on our own beliefs and experiences.

Ultimately for the purposes of this study, social constructivism best serves as the basis for the theoretical framework. This is because the focus of the study is on the function of the normative concept of learning EFL writing, and the learner's attempt to work out a solution to a practical problem, specifically, developing a critical, cross-cultural self. A constructivist account of a concept, unlike a traditional analysis, is an attempt to work out the solution to 
that problem. Social constructivism is therefore a valuable source of theoretical knowledge in this context, because it emphasizes how meanings and understandings grow out of a learner's social interactions.

\subsection{Devising the theoretical framework}

In constructivist thinking, learning does not happen in isolation. It is in the interaction with texts and other people-learners and teachers-that learning (knowledge) comes to exist. These interactions of sharing ideas are what establish a learning community where a student comes to understand the basis for her/his social and cultural identity. This is crucial to the fundamental belief of constructivist theory, that the inter-subjective sharing of ideas shape a person's behavior by constituting her/his interests and identity as a member and participant in an established community.

An EFL student-in varying degrees, depending on the teacher-is introduced to challenges to the student's social and cultural identity in the form of cross-cultural studies. University EFL students at the same time take on the challenges of the academic setting in which the contact with the other culture takes place. By combining the intercultural challenges with the academic challenges, the learner then constructs an identity that can be described as cross-cultural, and is then able to make cross-cultural arguments. 
These general concepts were used to develop the following diagram (Figure 1) of the theoretical framework for the study:

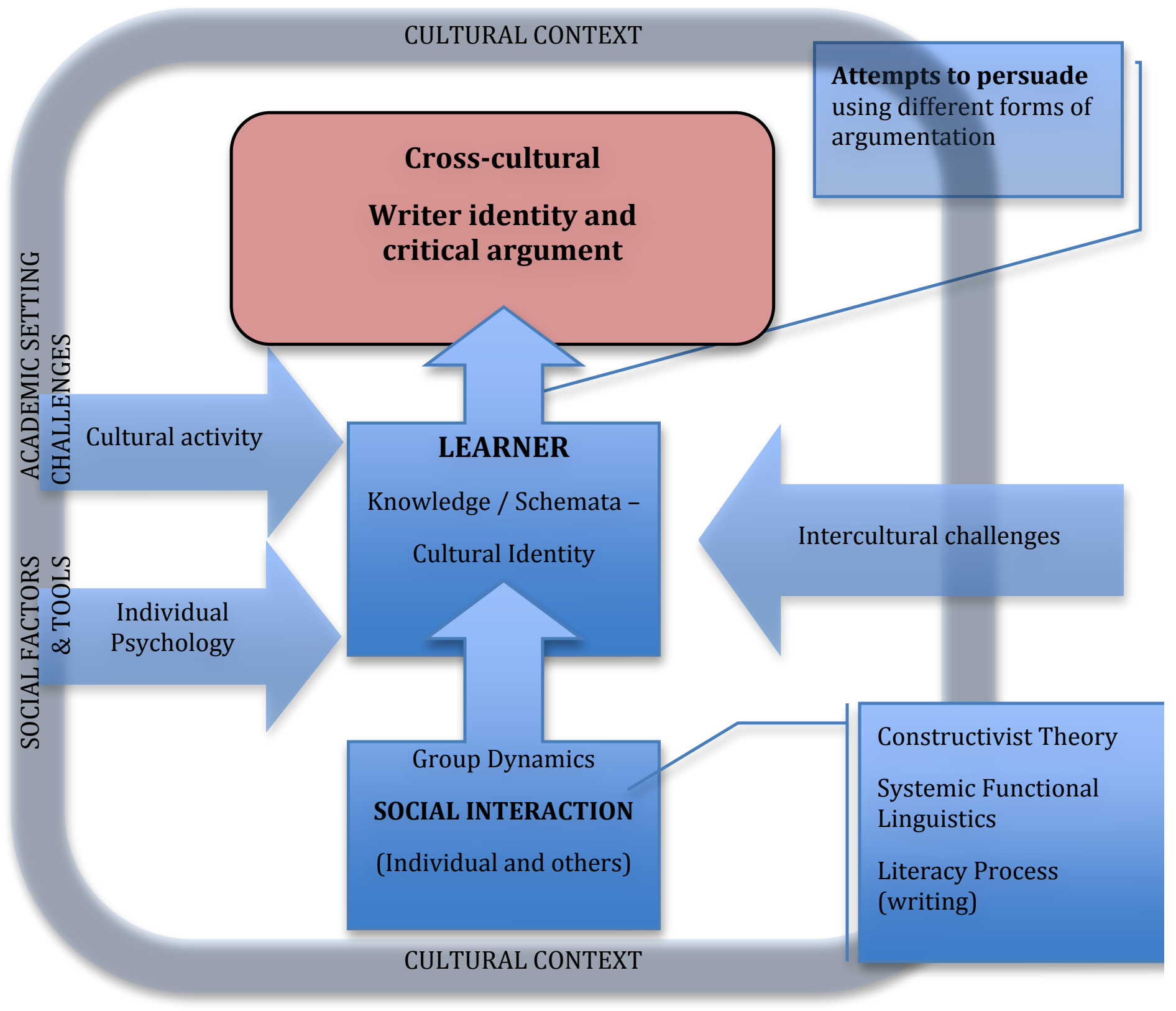

Figure 1: Theoretical framework of general concepts 
The diagram's focus is on the learner, specifically, the learner's knowledge, schemata, and cultural identity. Below that central shape in the diagram is social interaction between the individual and others (in pair and group work) as the basis for what creates the learner's awareness of all of these aspects. The movement from social interaction to the learner is labeled "group dynamics" as this is different to the "individual psychology" feeding in to the learner through social factors and tools prescribed by the learner's cultural context. The box to the right identifies the theory, linguistic focus, and process underlying the group dynamics, namely constructivist theory, systemic functional linguistics, and literary process. Also, feeding in from the cultural context is cultural activity manifested in academic setting challenges (what could be labeled "classroom dynamics"). The final influence feeding in to the learner is the intercultural challenge from outside the cultural context. In this study, this is the introduction of English as a foreign language/culture. All of this then gets developed as the learner makes attempts at persuasion through experimentation with the new language/culture in different forms of argumentation (usually by command of a teacher in a classroom or for an assignment). It is this attempt (labeled "attempts to persuade" in the box to the right) that, if understood by the learner to be an exercise in critical thinking, then leads the learner to establish a cross-cultural identity and the ability to develop a cross-cultural critical argument.

\subsection{The phenomenon of constructing cultural and academic writer identities}

The focus on identity construction in this study is socially co-constructed through written language use. Parallels can be drawn with the above model and Harre's (1984) fourquadrant model, which shows identity being constructed through four stages of social interaction (see Figure 2).

The idea behind Harre's model is that an individual first establishes an understanding of public conventions within her/his cultural community (quadrant 1). Next, the individual establishes a cultural identity and becomes a member of that community by "appropriating" the conventions of the cultural community (quadrant 2). This realization is 
then internalized personally in which an individual "transforms" her/his position in relation to others in the same community (quadrant 3). Finally, the individual displays her/his identity publicly through "publication", in the case of this study, through a writer identity (quadrant 4). In successful display of a writer identity, i.e. the reader can identify the writer as a member of that community, the final step in the process is where the individual confirms her/his identity and the social order of the cultural community through "conventionalization." This quadrant model therefore relates to Figure 1 in that the student writer goes through a process of establishing a cross-cultural identity and an ability to develop a cross-cultural argument through a process of social interaction and recognition of social and academic communities.

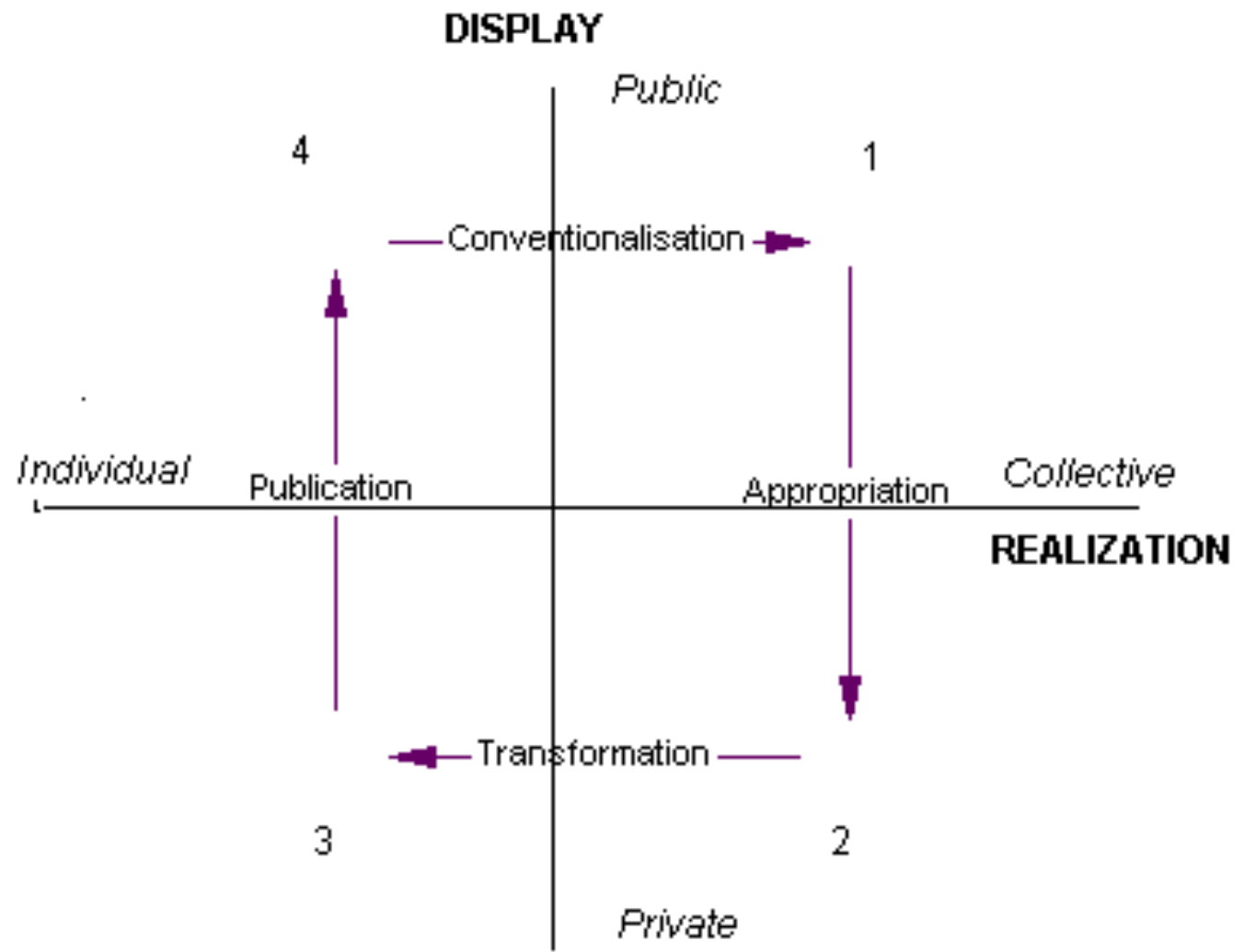

Figure 2: Harre's (1984) 4-quadrant model

The construction of cultural and academic writer identities occurs in social interaction in the cultural and academic communities. Gomez-Estern, Amián, Sánchez Medina and Marco Macarro (2010), state: 
[I]dentity is generated in social interaction, mediated by cultural instruments, and contextually situated. That is, individuals need to define themselves because they are immersed in social settings in which there is another (individual, social group, or culture). Cultural identity arises from the relationships that individuals maintain with cultural groups, with which they struggle and cooperate (Woodward, 1997). (p.232)

Should cultural identity be attributed to the individual or the group? Some assert that identity is found in a group, so an individual constructs her/his cultural identity within that group, known as a "collective identity" (Gomez-Estern, et al., 2010, p.233). This idea is the important for my theoretical framework as the many of the student participants developed their arguments in accordance with their peers.

The socio-cultural identity that this study focuses on is not objectivist or isolated, but instead looks at the mediation tools used by a collective community in a constructivist way-to construct an identity in a social setting. Within the specific focus on the development of academic literacy in the form of writing in a foreign language, it is understood that the use of the socio-cultural mediation tools by individuals within that setting influences their semiotic (language) choices (Wertsch, 1998). In this study, writing in English as a foreign language is a mediation tool developed in an individual's culturalhistorical psychology, which is used to construct one's identity. This is because writing (as a mediation tool) is orientated internally and externally at the same time (Bakhtin, 1986; Vygotsky, 1978).

After the development of a cultural identity comes the development of an academic writer identity. Ivanič (1998) extends the Systemic Functional Linguistics model of language use described by Halliday (1978; 1994) in order to explain how academic identity is constructed in written discourse. Halliday's model of language use showed that "ideational, interpersonal and textual meanings conveyed by language all contribute towards constructing an individual's identity" (Sokol, 2005, p.324). Ideational meaning refers to the individual's formation of ideas or concepts, and the interpersonal meaning refers to the individual's understanding of her/his position in relation to others. Ivanič (1998) uses this model to explain that these are precisely the points that establish a person's academic writer identity. 
An academic writer identity is made present in the writing in the form of various "selves" including autobiographical, authorial and/or discoursal (Clark \& Ivanič, 1997). The figure below shows the three subject positions or the possibilities for selfhood or selves of a writer that are affected by a socio-cultural context.

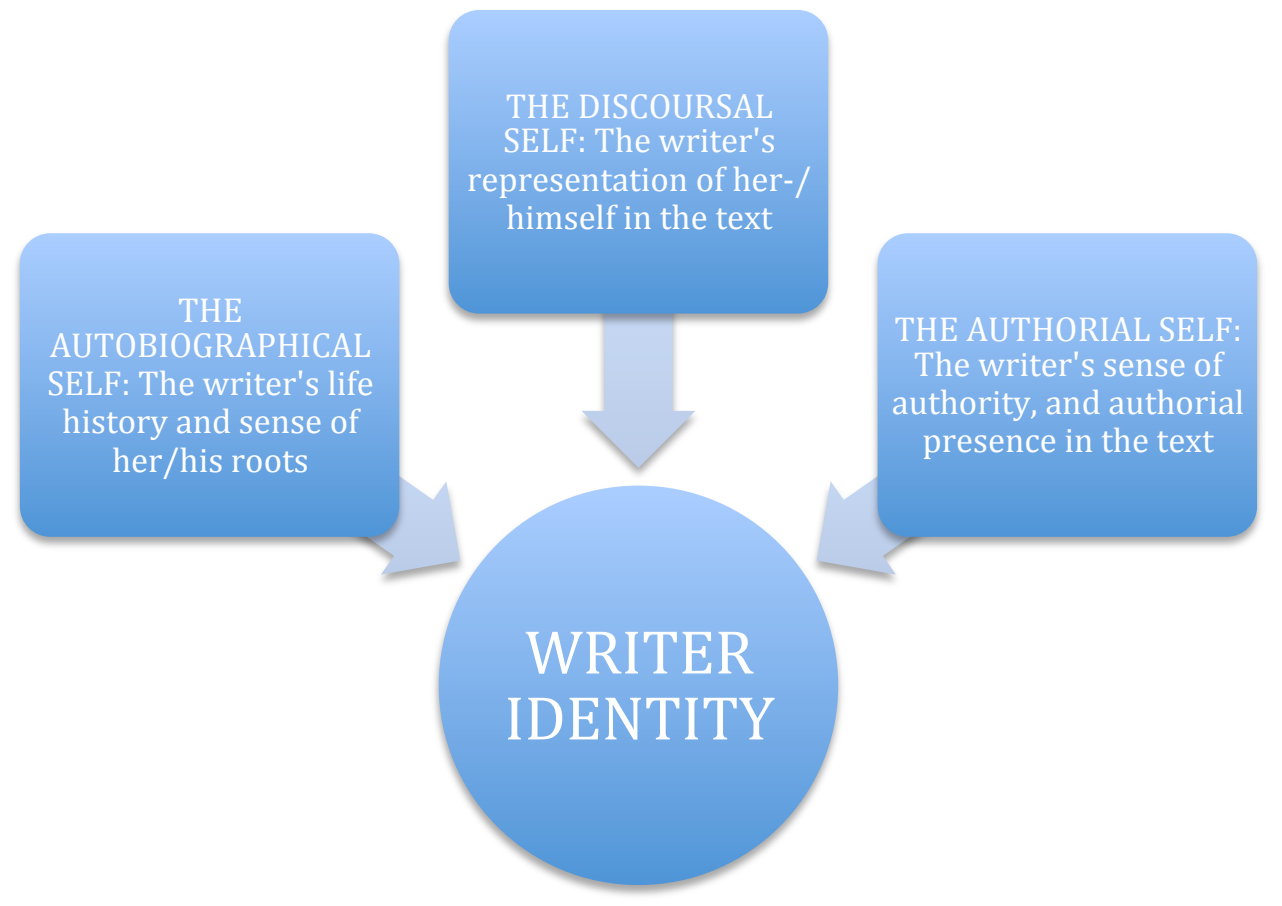

Figure 3: Subject-positions; socially available possibilities for self-hood (Clark \& Ivanič, 1997, p.137)

These 'selves' are utilized according to the writer, the task, and socio-cultural or sociopolitical aspect (Ivanič, 1998). The autobiographical self makes use of personal experience as evidence. The authorial self makes demands on the reader by asserting either personal or substantiated claims. The discoursal self takes an objective approach, with no personal language and attributing others in support of claims. These different selves are manifested in the writer's attempts to persuade the reader through different forms of argumentation, which takes place in the final step of establishing a writer identity (see Figure 1 above).

In academic writing, representing ideational and interpersonal meanings is manifested in the purposeful selection of a self or selves. In English L2 writing, this has particular implications in that the cultural identity of the writer could potentially interfere with the goals of his/her academic identity. A Japanese student writing in English L2 may have to make a conscious switch from a cultural identity that expects writing to be inductive to an 
academic identity that expects writing to be deductive (Noor, 2001, discussed in section 2.2.1). The meanings in the writing are expected to be equally acceptable for writing teachers as those of a native writer of English, therefore English L2 writers attempt approaches to writing that are more typical of native English writers. This is where students may attempt the Western quality of academic writing that Casanave (2002) describes as "playing the game". This game involves six strategies, raising important questions:

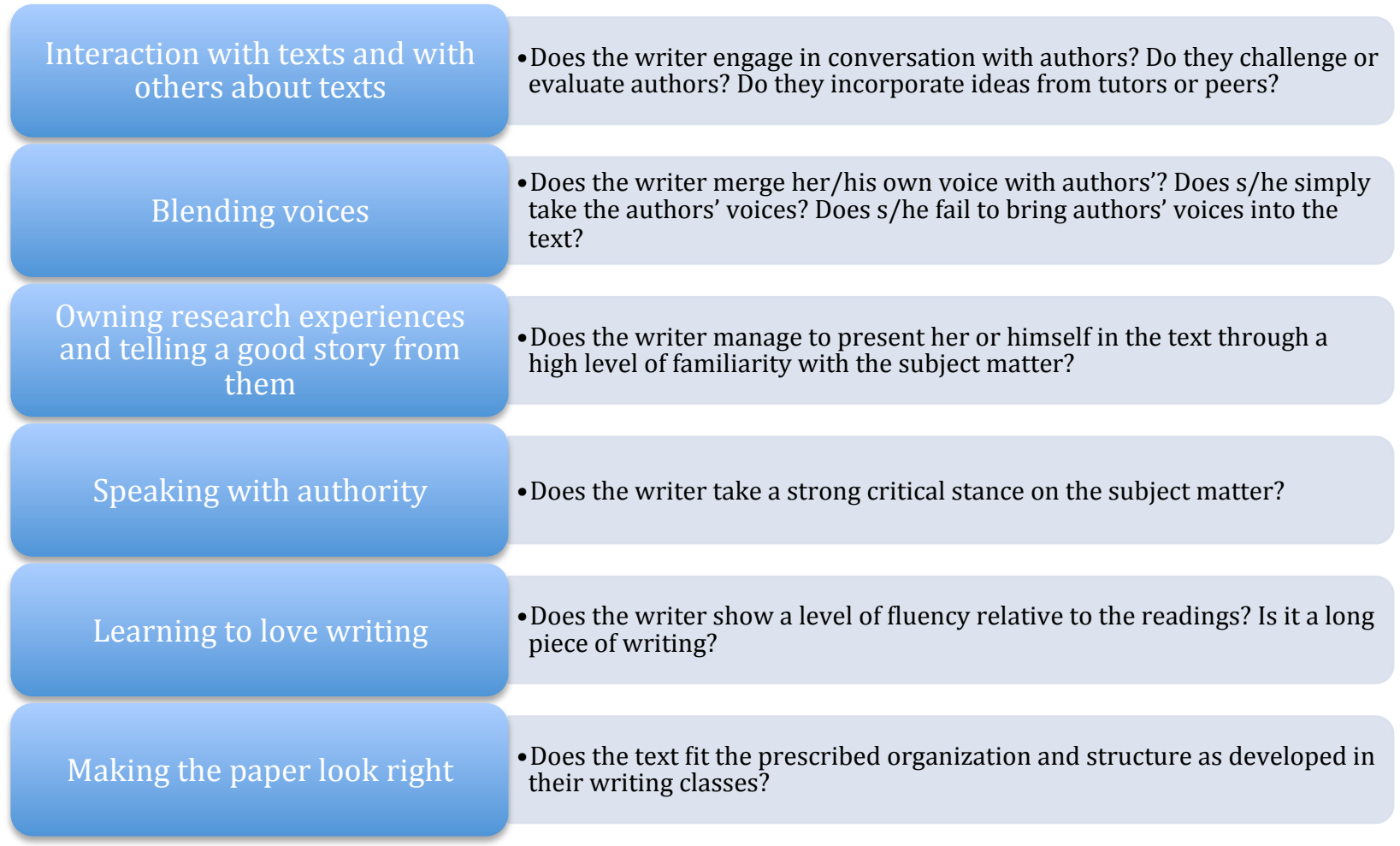

Figure 4: Writing Game Strategies (Casanave, 2002, pp.61-74)

Casanave's strategies are based on years of experience and research as an academic in the Japanese university English language-learning context, and thus provide an appropriate context-specific framework. Therefore they make a valuable addition to the theoretical framework in my study, which will be discussed in the following chapter on methodology.

Ultimately, the academic L2 student writer has much to negotiate in terms of determining how to establish her or his academic writer identity. All of these directly affect the writer's attempt to critically argue a thesis. 


\subsection{The phenomenon of developing critical argument in L2 writing}

When a student is given the task of presenting an argument in academic writing, a process is started. The argument goes through a series of stages, each one influenced by certain factors within the academic community. The process is shown in figure 5, following the description below.

- The first stage is the student's internalization of the task from the perspective of her/his cultural identity. This first stage allows the student to establish some semblance of a thesis that shows that $\mathrm{s} /$ he has something to say about a particular topic - the more familiar the topic, the easier this is to establish (Stapleton, 2001).

- The second stage is the framing of the argument by the academic genre presented by the writing teacher. For the student, this is the point at which the thesis takes a particular shape according to the genre, and the student may begin externalizing the argument through drafting.

- The third stage, which may or may not occur depending on the parameters of the task, is when the student does some research. The student explores a range of voices and perspectives on the topic in order to see how her/his thesis fits into that range. If the student goes through this stage, the drafting may start here.

- The fourth stage is when the thesis gets tested through peer reader and teacher feedback. The response from the audience allows the student writer to see how successful s/he was in shaping and supporting the argument in the draft.

- The fifth stage is the re-internalizing of the thesis by considering how the audience reception of the argument fits in with the student writer's cultural identity.

- The sixth stage is the adjusting of the thesis in order to meet the expectations of the academic community. This is done through successful revision of the thesis, where necessary.

- The seventh and final stage is the point in which the student presents a sound thesis and in so doing, establishes an academic writer identity. It is at this final point that the student has established not only what to say, but how to communicate the argument successfully. 


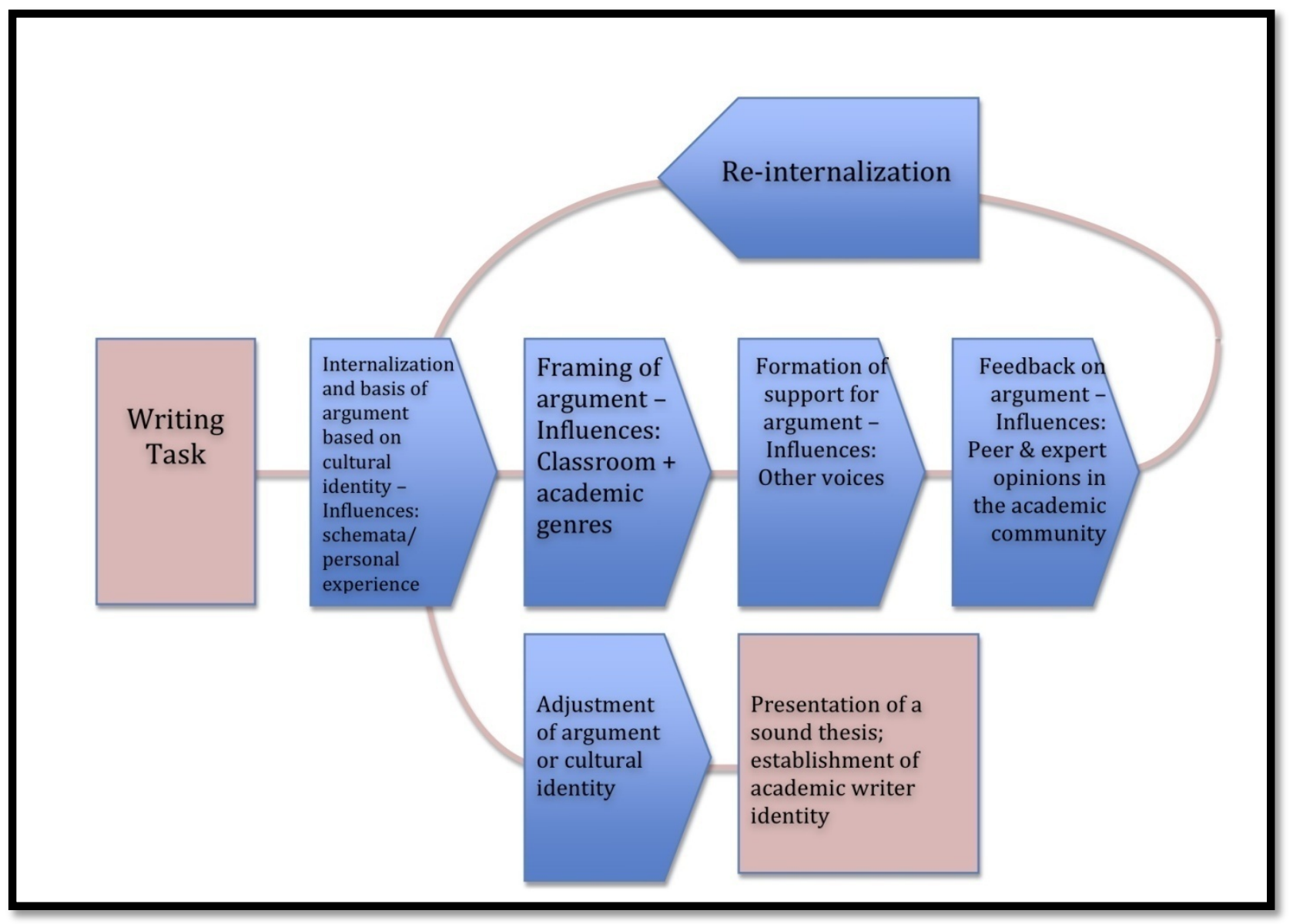

Figure 5: 7-stage development of critical argument

I will now explain in more detail some of the components that influence the development of critical argument, first in general theory, then in L2 writing. In the writing process, learners make attempts at persuading readers through different forms of argumentation by experimenting with ideas presented in English as a foreign language and culture. This is usually by command of a teacher in a classroom or for a writing task. The different forms of argumentation provide the learner with various approaches to persuading her/his reader. Clark and Ivanič (1997) suggest that as student writers negotiate the reader-writer relationship, they experiment with the language by taking on certain stylistic techniques in order to utilize the most effective identity or self to persuade their readers.

First, it is necessary to provide some general background on critical argument theory. The understanding of developing critical argument in this study sees argument as a social 
activity, essentially generating a discussion that serves to resolve some difference of opinion (van Eemeren \& Grootendorst, 1984). With this perspective, critical argument serves as a design theory in which rationality is immersed in social and political contexts. It serves as a means of evaluating opposing positions with the aim of gaining insight from them-generating a shared construction of knowledge-and avoiding any logical or rhetorical fallacies (van Eemeren \& Grootendorst, 1992). With this theoretical insight, this study serves to explore how student writers make arguments to generate a discussion that reflects the social and political context of the immediate writing task.

There are different "ways of arguing" that students may attempt, as defined by different disciplines and different writing tasks within those disciplines. Some disciplines or tasks may place more importance on synthesizing multiple sources than others that look more toward planning solutions. Hyland (2008) points out that this has pedagogical implications in that student writers need to be informed of the "purposes, genres and readers that ... students will need to communicate with" (p.105). Hyland emphasizes the importance of a "reader-oriented approach" that provides students with raised rhetorical awareness of the expectations on their writing within the genre of the specific task.

There are two approaches a student writer typically takes when presenting an argument, both of which have implications for the establishment of writer identity and the utilization of critical thinking skills. One is when the student writer "borrows" the argument from the relevant sources and uses that borrowed argument as the thesis that then gets developed. This way of arguing is fairly common as there is a general goal for EFL writers to make their writing appear "native" (Stapleton, 2002b). In this approach to developing critical argument in essay writing, EFL student writers will often mimic features from their sources, both of language and perspective (Masao, 1976, discussed in section 2.2.1). The problem with this practice is that it can lead to a loss of the writer's voice as well as an inability to display critical thinking skills. In contrast, the other way of arguing in which a writer essentially defends her/his own position on a topic requires the student writer to establish her/his own argument in the thesis that may or may not be based on ideas from outside sources (Stapleton, 2001, discussed in section 2.2.2). This approach requires 
student writers to make important choices that involve more critical reasoning in the development of their argument.

The focus on critical thinking in student writers' development of critical argument is crucial to this study. However, there has been well-documented deliberation on the value or damage of focusing on critical thinking in developing critical argument in writing classrooms (Benesch, 1991; Phelan, 2001). Atkinson (1997) points out that although there is some confusion among educators about how critical thinking is supposed to function in academic writing, it is nevertheless vital to success in higher education. Davies (2003, pp.12) states,

[S]kills in critical reasoning are as important for educational success as is mastering linguistic genres associated with particular fields of study and vice-versa-both skills are equally necessary for good academic performance: "Poor English and poor argument or analysis [are] inextricably linked” (Felix and Lawson, 1994, p. 67).

Davies (2003) continues, "For students, especially students from non-English speaking backgrounds (NESB) the "specter" of critical thinking, not writing, is usually their single greatest fear" (p.2). The implications of this for the study are significant in that if the specter of critical thinking is indeed non-English speaking students' single greatest fear, then the development of critical thinking is the ideal focus in an analysis of Japanese university students learning to write in English.

\subsection{Focus on critical thinking: Critical writing as mediated action}

As described earlier in this chapter, the development of social constructivist theory in L2 writing research led to a focus on student writers' shifts from internalizing to externalizing. Having completed the process of establishing and confirming cultural and academic writer identities, an individual is then able to use that knowledge/ awareness of the identities in relation to the academic community. Within the academic community the L2 student writer negotiates mediated action-often in the form of working with peer and teacher feedback as well as teacher instruction-and intercultural challenges in order to attempt to argue a point. Through additional feedback, the L2 student writer then has the ability to argue critically and persuade readers within the academic community. 
To explore Japanese university students' development of critical thinking in learning academic English writing, it is appropriate to focus on it as a form of semiotic mediation with consideration given to the "social, cultural, and historical situatedness of mediated action" (Wertsch, 1993, p.8). Wertsch (1993) explains that the notion of mediated action sees human action as inherently affected by social, cultural and historical aspects, and suggests using it as a "unit of analysis" (p.119) in social constructivist research. This unit of analysis allows me to conceptualize the identities of the student participants as active members of a social, academic community. It also allows me to focus on action mediated by tools (i.e. EFL writing) in order to analyze both the writer and the cultural instruments used in the writing as co-existing. Those tools shape the writer's act of identification. The focus on action is essential as it avoids the idea of identity formation represented by discourse, but rather looks at the social construction of that discourse. It is a particularly useful paradigm to see identity as a communicative action, as it allows for the understanding that identities can change depending on the activity (Ivanič, 1998).

The relationship of this concept of identity to critical argument is one of due process. In a situation where a learner is presented with reading and writing skills-in this study, in EFL-it is understood that this is only the beginning, and the end point is the mastering of the particular discursive instruments of "ways of arguing" (see "attempts to persuade" in Figure 1), which allow the learner to appropriate the cultural resources of the target language (Gomez-Estern, et al., 2010, p.238). This process outlines two steps: the first is the acquiring of new mediation tools in the target language to organize social interactions, which then leads to the second step of regulating ways of thinking in the target language. It is inevitable that when learners take on these new tools in another language, this will change their schemata and understanding of themselves and their own socio-cultural identities.

\subsection{Conclusion and research questions}

The fundamental link between all the theories in this study is the social constructivist understanding of an interpersonal collaborative construction of knowledge. Sociocultural 
theory, identity construction theory and critical argument theory are all crucial to gaining an understanding of the process of Japanese university students learning academic EFL writing. This is because it is necessary to examine the development of these students' ideas about critical thinking and how it is applied in the writing classroom as well as to their writing, with careful consideration of the students' socio-cultural contexts.

A brief summary of each of the main theories involved in the study follows:

Socio-cultural theory, as Lantolf and Thorne (2006) point out, clearly connects sociointeractional constructivist theory with a collaborative learning environment such as university EFL writing classrooms in Japan. It allows for the students' and teachers' social and cultural backgrounds and positionalities to inform the study in valuable ways, and to recognize the significance this has on Japanese university students establishing writer identity and developing critical argument in learning academic EFL writing.

Identity construction theory involves the formation of both cultural identity and academic identity. In cultural identity construction, social interaction is the basis for a person's understanding of her/his position in relation to others within the same cultural community. Identity construction theory helps in explaining the ideational and interpersonal relationships involved in forming an academic identity. Ivanič (1998) built on this theory to explain that the construction of academic writer identities is dependent on social and cultural factors and is developed in written discourse when a writer makes particular language choices in attempts to persuade her/his reader.

Critical argument theory maintains that argument generates a discussion in the shared construction of knowledge (van Eemeren \& Grootendorst, 1992). As it pertains to EFL writing, the theory builds on the idea that writers use different ways and forms of arguing. In the development of critical argument in written discourse, EFL student writers must exercise critical thinking skills. This is done in the reception of writing instruction in the classroom, and in the expression and development of the thesis in their written texts.

Having analyzed relevant literature and outlined the key theoretical frameworks for the study, I can now expand the general research questions raised at the end of the previous 
chapter to focus on the following specific research questions that are the focus of this study:

1. What is the current practice of English writing education in relation to critical argument and writer identity in a Japanese university?

2. What are teachers' goals for these writing courses?

3. What are teachers' identifiable cultural expectations of EFL writing?

4. What practices do teachers bring to the classroom to develop critical argument and writer identity in students' writing?

5. How effective are these practices in terms of students' writing output?

6. Do students recognize critical argument and writer identity as important to their advanced writing education?

In the following chapter on the methodology, I will describe the tools used to respond to the specific research questions presented above. 


\section{Chapter 4. Plans and paradigms: The methodological framework of the study}

This chapter will provide an outline of the methodology used in the study. First I will discuss the research paradigms in order to further explain the reasons behind the social constructivist approach described in the previous chapter. I will then give an overview of the research procedure, including discussion of the validity and reliability of the instrumentation of that procedure. A discussion of what data was collected and the frameworks used in the analysis of the data will follow. Finally I provide an explanation of the ethics involved in the data collection and dissemination of results.

\subsection{Research Paradigms ${ }^{3}$}

As explained in the previous chapter, central to this study is the concept that writing is a communicative act, situated in a social, cultural setting (Rinnert \& Kobayashi, 2001; Casanave, 2003). This socio-cultural environment impacts heavily on students' exposure to critical writing as well as their motivation to learn how to write in the L2. It is the intention of this study to take an embedded case study approach to gain an understanding of the students', teachers' and researcher's socio-cultural positionalities in order to provide a deeper analysis in drawing its conclusions. The congruence of multiple socio-cultural positionalities has significant implications for the study, as there is some argument that it has the potential to invalidate my research. However, I will show that according to the philosophy of the study, this congruence will in fact inform the research.

In a case study research project such as this one where I, as a Western researcher, am observing Japanese university students in their own classrooms in Japan being taught critical English writing by either Japanese or Western teachers, certainly the issue of cultural sensitivity is crucial. It is the researcher's duty to try to be as objective as possible to be able to have a better understanding of the social phenomenon that is learning English writing in a Japanese university. One idea is to try situated qualitative research-to try to understand the situation from the students' point of view (Atkinson, 2005). Another idea is

\footnotetext{
${ }^{3}$ Much of the text in this section appeared in my article: A Western researcher in a Japanese university writing classroom: limited or advantaged? Journal of Asia TEFL 2(3). 139-146. Fall 2005.
} 
to remove myself from my own prior knowledge and prejudices. As a research methodology, this innocence (or ignorance) is generally never really possible, since my own life experience will always affect my understanding of what I observe (Berger \& Luckmann, 1966). Both of these ideas lead to dilemmas of great philosophical proportions - of trying to understand a cultural scene that is not my own. Ryuko Kubota, a Japanese professor of English linguistics in the US, offered me this advice via e-mail:

I think it's impossible to pursue an objective representation of social phenomena. Even if you were Japanese, you would have a certain social, cultural, and economic status that functions as a lens through which you interpret the data in a certain way. So rather than worrying about objectivity, I would expose my own subjectivity and explore what influence it might give to my data collection and interpretation. (R. Kubota, personal communication, Oct. 12, 2004)

Therefore, my subjectivity is pivotal in my own research since it ultimately defines the socio-cultural boundaries or positionality of any observational study I do.

This section will first provide a discussion on the issue of positionality, i.e. my position as a Western researcher in a Japanese context, in order to emphasize the point that by establishing my position as an outsider, I am problematizing meaning in order to inform the study rather than invalidate it due to bias. This is followed by a brief introduction to Grounded Theory as a fundamental qualitative research approach for my study. SFL and the Appraisal framework are then described as providing categorical parameters for the data. An outline of naturalistic inquiry as the ethnographic approach in my study is then provided before finally discussing the reasons behind taking a qualitative approach in this study.

\subsubsection{Problematizing meaning and socio-cultural positionality}

This type of qualitative research problematizes meaning in that it questions concepts and methodological implications in the creation or development of meaning through research (Jaffe \& Miller, 1994). In my research, the "problem" of meaning is treated by the issues brought up in previous studies that suggest Japanese university students are not able to apply critical thinking to their writing (Stapleton, 2002). The meaning of "critical thinking" is a problem that I believe has particular implications for Japanese students and it is these implications that I needed to familiarize myself with in order to conduct research in a 
Japanese university English writing classroom (Silva, 2005).

Positionality is crucial to the understanding of how meaning is problematized in my study. It is how I identify myself in terms of my sense of where and to what I belong or do not belong, and the social relations that are affected by this (Anthias, 2002). From an objectivist/positivist perspective (that all reality is objective and external to the mind and that knowledge is reliably based on observed objects and events), the concepts of positionality and structural embeddedness, have the potential of invalidating my research because my position as an outsider and participant within the observed target context would present a bias and be seen to alter the natural occurrence of events (Jaffe \& Miller, 1994). However, from a constructivist standpoint (to discover the ways that individuals and groups create their perceived reality) these concepts can rather be seen to inform the study (Berger \& Luckmann, 1966; Flowerdew, 2005).

My positionality as a Western researcher and native user of the English language is quite different to the students' positionality as Japanese learners attempting to cross culture and display critical thinking in their writing of English, a foreign language. Culture and language barriers need to be considered and recognition of these barriers as limitations of my research project is essential. Also, my positionality as a teacher is one of challenging students and questioning what are commonly accepted, key aspects of critical thinking. The positionality of students in Japan is traditionally quite opposite to mine, in that authority is

not meant to be questioned, although more recent research suggests that traditional understanding of Japanese students is no longer valid (Stapleton, 2002a).

This study incorporates essential exploration into the socio-cultural positions of Japanese university EFL students, their teachers, and myself as a Western researcher. Thus, it serves to reveal valuable insights and steps toward finding a solution to the problem of how to develop Japanese university students' critical thinking through EFL writing education.

\subsubsection{Grounded Theory}

In line with a qualitative research approach, my study utilized Grounded Theory (GT) in order to allow theory to generate (Glaser \& Strauss, 1967). GT stipulates that rather than starting with a hypothesis and then testing it, data are collected through multiple methods, 
which will be described in the next section (4.2). The data collected are grouped into similar concepts in order to allow for more categorical and workable data analysis. The categories in my study were shaped by the emerging theory in line with SFL and the Appraisal framework.

In order to guide research, researchers taking a GT approach will often use "sensitizing concepts," referring to non-definitive concepts that offer suggested directions for analysis, as opposed to definitive concepts that establish fixed frameworks (Glaser, 1978). Qualitative social researchers see sensitizing concepts as starting points for research, "draw[ing] attention to important features of social interaction and provid[ing] guidelines for research in specific settings" (Bowen, 2006, p.3). For my study the sensitizing concepts were not refined, but rather served as the foundation for the data analysis. These particular sensitizing concepts were gained through my two years experience as a university English composition teacher in Japan. In that time I had established two concepts: 1) I familiarized myself with the debate on Japanese students' critical thinking ability, and 2) I found a way to not only acknowledge but also work with the cultural and language barriers between the participants and myself, discussed in section 4.1.1. At first, the loose or "fractured" concepts were later combined through "theoretical coding" to construct a theory to explain the participants' main concerns (Glaser, 2004). This generated theory was eventually incorporated when forming the codes for the analysis of the data.

\subsubsection{SFL and the Appraisal framework}

Systemic Functional Linguistics (SFL) is an ideal approach for this study because it is functional, i.e. it serves to explain activity and purpose relating to the way language works. SFL maintains the view that language is communicated in the social contexts in which it is used and cannot be understood separately from them. Therefore language is inherently a social phenomenon. It sees language as a system of choice, that is, it is viewed as a variety of particular lexical and syntactic choices exercised in order to realize a particular meaning (Martin, 2000).

In SFL theory, the maximal unit of analysis used is the text-in the case of this study, the students' written texts. SFL maintains that a text is a piece of language in use. That text 
occurs in two contexts, one within the other. First is the cultural context, which includes the purposes, attitudes, values and shared experiences of people living in a particular culture, established in interactions with others who share the same cultural context (Paltridge, 2002, p.45). The second context is the subject-specific context in which purposes, attitudes, values and shared experiences may be different-in the case of this study the second context is intercultural-that of EFL. These two contexts (cultural and intercultural) are shown in Figure 1 in section 3.3.

It is necessary at this point to make a distinction between Systemic Functional and ethnographic approaches for the purposes of this study. This distinction is made because SFL derives all culturally relevant information mostly from the text itself, whereas the ethnography goes beyond the text into examinations of the social and cultural context in which the genre occurs to explore "'insiders' views' on the genre" (Paltridge, 2001, p.46). Today, prominent genre scholars combine the two approaches, usually during the contextual analysis phase of genre research (Bhatia, 1993; 2002). The ethnographic approach in this study is critical in that it explores students' social and cultural contexts in order to gain an understanding of the relationship between students' motivation and thinking, and motivation and the ability to make claims in the form of theses. These are particular advantages of using a social constructivist ethnographic approach (Carspecken, 1996). Also, using an ethnographic approach allows for interpretive and qualitative examination of learning in a naturally occurring setting (Hyland, 2006). Since SFL emphasizes connections between texts and social contexts, a better understanding of those social contexts can be gained through an ethnographic approach.

SFL specifies a set of grammatical systems used to produce meaning with language; in other words, it provides a detailed schema of how language works. The particular SFL schema my study is using is Appraisal Theory-one that offers a framework for exploring the way writers use language to evaluate, take a stance on a topic, construct a writer identity, and manage the reader-writer relationship (Martin \& White, 2005). This schema is layered, and stretches from the cultural and immediate social situation to the actual language being used in whole texts to the micro levels of written language structure, such as word choice. The appraisal framework is a tool for categorizing language choices. There 
are several key concepts in SFL deployed to explore discourse. For this study, Appraisal is to be used as a system to uncover interpersonal meaning-attitudes negotiated in the texts, the strength of the feelings involved and the approaches the writers take to sourcing values and directing their readers (Martin \& Rose, 2003). The language features within the Appraisal framework will be explained later in the following section (on text analysis).

The importance of the current study is that it is classroom-focused, allowing Western Japanese university teachers' and Japanese students' own perspectives to be used by a Western researcher to better understand their socio-cultural/ socio-political environment. The focus of the analysis from the perspective of SFL (looking at language as a resource for making meaning) brings Western linguistic perspectives into the Japanese learning environment, a relationship that has not yet been fully developed in a research project such as this. The structural embeddedness in my study is the relationship of the students' experiences with learning English writing and the broader social context of the expectations and understanding of the curriculum at their university in Japan. The broader social context controls these expectations and this understanding, which is why researchers in this area are looking more towards social and political aspects to be taken into consideration in second language writing pedagogy, particularly in Japan (Casanave, 2003). Earlier studies (Inoue, 1997; McFreely, 1999; Yoshimura, 2001) criticized the suggestion that Japanese university students are limited by this structural embeddedness and therefore cannot produce critical thinking in their writing. In an attempt to avoid such pitfalls in my study, I decided on the ethnographic approach of naturalistic inquiry.

\subsubsection{Naturalistic Inquiry}

In line with social constructivist theory, this study incorporated the ideologies of naturalistic inquiry (Lincoln \& Guba, 1985). The naturalistic paradigm incorporates five basic beliefs about research. They are: 1) realities are multiple, constructed and holistic; 2) the inquirer and the "object" of inquiry interact to influence each other and are inseparable; 3) "working hypotheses" are developed for purposes of generalization; 4) causes and effects are indistinguishable as all entities are mutually simultaneously shaping each other; and 5) inquiry is bound in values (Lincoln \& Guba, 1985, pp.37-38). There are fourteen 
characteristics of operational naturalistic inquiry (Lincoln \& Guba, 1985, pp.39-43); the presence of these characteristics in my study is shown below:

1. Natural Setting - Observations were done in the classroom, and interviews were held at the university.

2. Human Instrument - Other than students' written text, all data was gathered from the students and teachers by the researcher.

3. Use of Tacit Knowledge - The researcher's experience teaching in Japan and Australia offered intuitive knowledge.

4. Qualitative Methods - This was necessary to show depth of exploration and compatibility with research in this area.

5. Stratified Purposive Sampling - 4 teachers and 16 students (2-4 from each of 6 classes) were selected to form the embedded case studies; the students represented a range. Details appear in the following chapter.

6. Inductive Data Analysis - Multiple realities were expected to be discovered; conclusions offer only analytical generalizations.

7. Grounded Theory - Theory has been generated in relation to data gathered from students and their teachers. Details appear in the following chapter.

8. Emergent Design - The particular area of focus or direction of study changed as new areas were discovered in data collection, e.g. rather than focusing on how students learned how to write argument essays, since not all teachers had such tasks, I had to focus on aspects of argumentative writing such as the communicative aspect of writing (acknowledging the reader) and generating an opinion (developing the thesis).

9. Negotiated Outcomes - Interviews were reliant on informants' perspectives.

10. Case Study Reporting Mode - Data is presented as multi-method embedded case studies. 
11. Idiographic Interpretation - Interpretation of data has been done on individual responses and conduct and does not make references to Japanese university students in general.

12. Tentative Application - Theory about problems students experience with English writing may be applied to considerations of policy and curriculum design.

13. Focus-determined Boundaries - Like emergent design, the boundaries of the research changed as new areas of focus emerged.

14. Special Criteria for Trustworthiness - An area of particular importance to the outcomes of this study - validity and reliability are discussed in length.

\subsubsection{Why a qualitative approach?}

Qualitative research and quantitative research are considered distinctly opposite in nature (Crotty, 1998). However, the distinction should be made at the level of methods, and not at the higher level of epistemology or theoretical perspective. Most research textbooks describe quantitative research as empiricist and positivist, while qualitative is nonpositivist. But we are reminded that any research can, without problems, incorporate both methods. Qualitative research by its nature incorporates multiple methodologies. It allows for interpreting natural phenomena in terms of the meanings people bring to them.

Although some quantitative data were collected with initial questionnaires for strategic purposeful sampling, I decided that my research questions would best be answered with qualitative data, rather than quantitative, because "they are a source of well-grounded, rich descriptions and explanations of processes in identifiable local contexts" (Miles \& Huberman, 1994, p.1). As well-grounded, rich descriptions, the data I collected offer a depth of perspective in an area that has otherwise been limited to generalizations based on more quantitative-based research (Liebman, 1992; Deckert, 1993; Janopoulos, 1992).

As a quantitative study my topic could have been conducted using large-scale surveys, in which questionnaires would utilize scales for students and teachers to rate their experiences with the learning of English writing. Observed classroom interactions and responses in interviews could have been coded in order to conduct a statistical analysis. 
The advantages of a quantitative study on this topic would be the opportunity for statistical generalizability as well as the straight-forwardness of the methodology. However, the disadvantages far outweigh the advantages. A quantitative study would not allow for the invaluable depth and variation of my own interpretation. As a set of case studies, the data collected needs to be verbal and descriptive, not numerical. This study is on people's opinions, which cannot be fully understood if compartmentalized into numbers on a scale. It is a case of breadth versus depth. My study was therefore best suited to a qualitative approach. I was able to collect data and interpret that data in my analysis through a range of considerations and spectrums.

Murphy \& Longino (1992) suggest that there is a "theoretical shift" from quantitative to qualitative inquiry and that this shift is focused on the idea that symbolism is representative of everything social. This supports the utilization of qualitative research methods in a study such as mine in that students' writing is essentially symbolic of their understanding of expectations and applications of English writing, which is considered a social act in a socio-political aspect (Casanave, 2003).

\subsection{Research procedure}

The data collection methods for this study included classroom observations analyzed using a flexible ethnographic approach, and an analysis of students' written texts, with participant interviews used to support the data. Additionally, regular interviews were conducted with the teacher and student participants. In order to inform initial observations and interviews, course syllabuses were collected for each course. For subsequent interviews, course materials such as handouts were also collected in order to discuss issues related to them. The three methods of data collection provide data triangulation (the use of more than one method of data collection), a valuable strategy to augment the thoroughness of the research and counter threats to validity (Denzin, 2009). These research methods were used to answer the six research questions as outlined in the following table: 
Table 1: Summary of data collection instruments and analysis, adapted from Miles \& Huberman (1994)

\begin{tabular}{|c|c|c|c|}
\hline PURPOSE & RESEARCH QUESTION & $\begin{array}{l}\text { INSTRUMENT } \\
\text { OF DATA } \\
\text { COLLCTION } \\
\end{array}$ & TYPE OF ANALYSIS \\
\hline \multirow{2}{*}{$\begin{array}{l}\text { To } \\
\text { investigate } \\
\text { exploratory }\end{array}$} & \multirow{2}{*}{$\begin{array}{l}\text { 1. What is the current } \\
\text { practice of English writing } \\
\text { education in relation to } \\
\text { critical argument and writer } \\
\text { identity in a Japanese } \\
\text { university? }\end{array}$} & Observations & \multirow{2}{*}{$\begin{array}{l}\text { Content analysis: Teachers were } \\
\text { asked to explain their } \\
\text { understanding of current } \\
\text { approaches, confirmed through } \\
\text { classroom observations. }\end{array}$} \\
\hline & & $\begin{array}{l}\text { Teacher } \\
\text { interviews }\end{array}$ & \\
\hline \multirow[t]{2}{*}{$\begin{array}{l}\text { To identify } \\
\text { exploratory }\end{array}$} & \multirow[t]{2}{*}{$\begin{array}{l}\text { 2. What are teachers' goals } \\
\text { for these writing courses? }\end{array}$} & $\begin{array}{l}\text { Course } \\
\text { syllabuses }\end{array}$ & \multirow{2}{*}{$\begin{array}{l}\text { Content analysis: After examining } \\
\text { the course syllabus, the teachers } \\
\text { were asked to explain their } \\
\text { philosophy behind the goals of } \\
\text { the course(s). }\end{array}$} \\
\hline & & $\begin{array}{l}\text { Teacher } \\
\text { interviews }\end{array}$ & \\
\hline \multirow[t]{3}{*}{$\begin{array}{l}\text { To generate } \\
\text { exploratory }\end{array}$} & \multirow{3}{*}{$\begin{array}{l}\text { 3. What are teachers' } \\
\text { identifiable cultural } \\
\text { expectations of EFL writing? }\end{array}$} & $\begin{array}{l}\text { Teacher } \\
\text { interviews }\end{array}$ & \multirow{2}{*}{$\begin{array}{l}\text { Content analysis: All participants } \\
\text { were asked to explain their } \\
\text { understanding of writing } \\
\text { expectations and feedback. }\end{array}$} \\
\hline & & $\begin{array}{l}\text { Student } \\
\text { interviews }\end{array}$ & \\
\hline & & $\begin{array}{l}\text { Students' } \\
\text { written texts }\end{array}$ & $\begin{array}{l}\text { Text analysis: Students' attempts } \\
\text { to meet teachers' expectations } \\
\text { were analysed according to } \\
\text { specific language choices. }\end{array}$ \\
\hline \multirow{2}{*}{$\begin{array}{l}\text { To identify } \\
\text { exploratory }\end{array}$} & \multirow{2}{*}{$\begin{array}{l}\text { 4. What practices do } \\
\text { teachers bring to the class to } \\
\text { develop critical argument } \\
\text { and writer identity in } \\
\text { students' writing? }\end{array}$} & Observations & \multirow[b]{2}{*}{$\begin{array}{l}\text { Content analysis: Classes were } \\
\text { observed for writing activities } \\
\text { that focused on argument and } \\
\text { identity; teachers were asked to } \\
\text { explain their reasons for the } \\
\text { activities. }\end{array}$} \\
\hline & & $\begin{array}{l}\text { Teacher } \\
\text { interviews }\end{array}$ & \\
\hline \multirow[t]{2}{*}{$\begin{array}{l}\text { To explore } \\
\text { exploratory }\end{array}$} & \multirow[t]{2}{*}{$\begin{array}{l}\text { 5. How effective are these } \\
\text { practices in terms of } \\
\text { students' writing output? }\end{array}$} & $\begin{array}{l}\text { Students' } \\
\text { written texts }\end{array}$ & $\begin{array}{l}\text { Text analysis: The "self" used in } \\
\text { students' writing was noted, the } \\
\text { number of Appraisal words used } \\
\text { were counted, and the writing } \\
\text { game strategy used was noted. }\end{array}$ \\
\hline & & $\begin{array}{l}\text { Student } \\
\text { interviews }\end{array}$ & $\begin{array}{l}\text { Content analysis: Students were } \\
\text { asked to explain their decisions in } \\
\text { their writing. }\end{array}$ \\
\hline $\begin{array}{l}\text { To explain } \\
\text { explanatory }\end{array}$ & $\begin{array}{l}\text { 6. Do students recognize } \\
\text { critical argument and writer } \\
\text { identity as important to their } \\
\text { advanced writing education? }\end{array}$ & $\begin{array}{l}\text { Student } \\
\text { interviews }\end{array}$ & $\begin{array}{l}\text { Content analysis: Students were } \\
\text { asked to explain what they felt } \\
\text { was important to their writing } \\
\text { education. }\end{array}$ \\
\hline
\end{tabular}

The study was conducted over a period of one academic year consisting of two 14-week semesters. All classes were held once a week for one class period of 90 minutes. Classroom 
observations were audio recorded and conducted once a month in each of the six classes for a total of seven observations for each class, and interviews were audio recorded and conducted with each participant once a month during the academic year, plus at least one follow-up interview for a total of eight interviews. Students' texts collected for analysis (one major work from each student) were all selected from the second semester, after students learned the necessary skills to complete the writing task.

\subsection{Instrumentation and validity of data collection}

Although there are arguments both for and against flexible and rigid instrumentation in case study approaches, careful instrumentation can increase a study's internal validity, generalizability and manageability of data and results. Miles and Huberman (1994) state: "[multiple-case studies] look forward to cross-case comparison, which requires some standardization of instruments so that findings can be laid side-by-side in the course of analysis" (p. 34). Regarding comparability of the study, they argue:

Using the same instruments as in prior studies is the only way we can converse across studies. Otherwise the work will be non-comparable, except in a very global way. We need common instruments to build theory, to improve explanations or predictions, and to make recommendations about practice. (Miles \& Huberman, 1994, p. 35)

My study had fairly focused research questions and a well-bounded sample of participants, for which a well-structured instrument design is suggested (Miles \& Huberman, 1994). Therefore the instrumentation for this study was relatively well structured prior to investigation to include classroom observations, interviews with both teachers and students, and analysis of written texts. The study applied both qualitative research methods (classroom observations and interviews) and quantitative research methods (analysis of students' texts). In the following sections, the two qualitative methods will be described first, followed by the quantitative method.

\subsubsection{Observations}

Classroom observations were made approximately once a month during the year-long course resulting in a total of seven for each class. All observations were audio-recorded and transcribed. The observations made were unobtrusive classroom observations, which 
meant that though I was present in the class, I drew no unnatural attention to myself, thus allowing the class to be conducted as if I were not there. This approach is most appropriate in the exploratory phase of a study to find out what is actually happening in a particular situation (Robson, 2002), in this case in an EFL writing classroom. The advantage of conducting observations is that I was able to watch and record (note form, in a field journal) the teachers' approaches to teaching English composition and students' reception of those approaches along with their behavior during classroom activities. Through this flexible ethnographic approach, I collected data by focusing on common behaviors and events. Depth (description and interpretation) was stressed over breadth, as there were a relatively small number of participants (Atkinson \& Hammersley, 1994).

The ethnographic approach allows theory to emerge through developing an understanding of shared cultural meanings of classroom procedures and practices - something that can only be understood through observation and study of the group in its natural setting, with an insider's perspective (Robson, 2002). There were many considerations about setting up the observation situation, especially acquiring the appropriate permission, and establishing observable frameworks. Also, it was important to establish early in the study why I selected to observe specific points and what I would do with the data once it was collected. Silverman (2001) outlines these questions in his distinction between ethnography and observation. The distinction is described as that "something extra" (i.e. the study of culture) social scientists do with their observations: they write ethnographies.

Ethnography does not always involve observations, but for my study observations were essential in order to answer critical research questions. To get deep enough into an understanding of the specific issues, anthropologists recommend that the ethnographic researcher needs to participate in the observation, rather than keep a distance. More than just a method, participant observation is best described by Atkinson and Hammersley (1994, p.249) as "a mode of being-in-the-world, characteristic of researchers" (italics and comma mine). As a participant observer the researcher should be able to come to understand and apply perspective, description, contextualism, process, flexible research designs and avoid early use of theories and concepts (Silverman, 2001). With this in mind, although the observations were designed as unobtrusive, I ultimately readied myself to 
participate whenever classroom activity naturally called for it, doing my best to do so without skewing data of the observed processes.

This flexible ethnographic approach gave me the ability to participate within the target context, allowing more natural actions. If the teacher asked me to participate in classroom activities by answering a question, I answered it. If a student asked me a question during student discussion activities, I answered it. I did not interject or ask questions myself at any time. I did not refuse to answer questions. I did not assist with a class in any other way. It was important for the people in the classroom to recognize my presence. It would have been very unnatural for a class to be conducted while attempting to ignore my existence in the room.

Additional flexibility with the observations was exercised in terms of the observation schedule, which is one of the reasons why there was a total of seven observations for each class instead of the possible eight; other reasons include the cancellation of classes for one week due to a measles outbreak, and the fact that classes start halfway through the first month and end halfway through the last month. Therefore the observation schedule was not fixed in advance, but rather decided according to those lessons that should contain more of what this study was aiming to observe. In order to prevent teachers from teaching to my research interests, the study was not described explicitly before observations.

There are particular criticisms of the validity of such a qualitative research method as observation. In order to avoid the idiosyncratic nature of self-observation, I did not conduct research involving my own teaching or my students' learning. Triangulation of data was accomplished through multiple methodologies, as opposed to combined levels or additional investigators. Spindler and Spindler (1992) propose several characteristics of effective ethnographies, including giving the observations contextual relevance, allowing the hypotheses to emerge in situ, and making observations prolonged and possibly repetitive. Also, in interaction with participants, an effective ethnographer uses inferences from observation to address insiders' views of reality, elicits participants' socio-cultural knowledge, explains the ethnographer's presence and describes personal, social and interactional position in the situation. 
Additionally, limitations to the observations in this study include possible discrepancies between accounts of classroom activities made by myself, teachers and students due to differences in perspective (LeCompte \& Preissle, 1993). To help ensure that I attained a pure data sample from L2 writing activities that occurred in my absence, written work from student participants throughout the semester of study was collected and discussed in interviews to provide evidence of the types of writing activities that were actually occurring day-to-day in the unobserved classroom. These writing activities were then compared to those given by the teacher in the observed classroom to highlight any discrepancies.

\subsubsection{Interviews}

In order to better inform the data from the observations and text analysis, semi-structured interviews with teachers and students were conducted approximately once a month during term, for a total of seven interviews with each participant. All interviews were conducted in English. Participants were given the option of conducting interviews in either English or Japanese, and all participants chose English. The students and two Japanese professors in this study were either bilingual or had a relatively high level of English proficiency. They indicated that they preferred English because it gave them the ability to co-construct knowledge with me, a native speaker of English, as well as the ability to be frank, and also as a chance to use English. This choice of language is indicative of my social constructivist approach.

Talmy and Richards (2011) emphasize the importance of taking a discursive perspective in theorizing interviews so that they can be critically and reflectively adopted in applied linguistics research. They explain:

the interview is conceptualized explicitly as a socially-situated 'speech event' (Mishler 1986), in which interviewer(s) and interviewee(s) make meaning, coconstruct knowledge, and participate in social practices (Holstein and Gubrium 1995, inter alia). (Talmy \& Richards, 2011, p.2)

They go on to explain that this discursive perspective is in line with Holstein and Gubrium's (1995) explanation of the active interview, the approach taken in this study (described below). This strongly supports the conducting of all the interviews in English. 
All interviews were audio recorded and transcribed. Transcription was done using an Interpretative Phenomenological Analysis (IPA) approach, which focuses on content and meaning rather than active language use (Reid, Flowers \& Larkin, 2005). IPA is ideal for my study as it seeks to understand students' experiences with learning to write in English at university, and their attempts to make sense of those experiences. It is also consistent with the theory-generating approach of Grounded Theory (discussed in section 4.1.2) in that data were collected with the intention of generating codes, rather than applying codes from pre-existing theory to the data. In line with an IPA approach to the transcriptions, aspects of paralinguistic (pronunciation, intonation) and extralinguistic (body language, gestures) were not marked in order to focus on the content of the interviews.

When structuring the framework for the interviews, there were a number of factors I had to consider to ensure successful and objective data collection. A first consideration was the style of interview. Data were gathered through active interviewing with participants. The interviews were semi-structured with several questions prepared, and they were flexible to allow me to respond as an active listener (Kaufman, 1994) with appropriate and valuable follow-up probe questions.

The process of active interviewing allows themes to develop from ideas grounded in the informants as well as ideas generated in the interviews (Holstein \& Gubrium, 1995). Active interviewing is a theoretical position that takes into consideration that the topic of study informs the methodologies, and the methodologies ultimately inform the potential of the phenomenon; in the case of this study, the phenomenon of a Japanese university student learning English academic writing in Japan. In much the same way, the phenomenon could also shape the methodologies (Silverman, 2001). Therefore active interviewing is the most appropriate approach to studies in which theory is to be generated.

What the active interviewing approach provided for my study was important flexibility. Although I had several questions prepared for each interview, often I followed up participant responses with additional questions in order to allow for potential ideas to emerge. Some of the ideas were then added to later interviews with all participants. Therefore the questions asked in interviews were only prepared after the previous set of 
interviews were complete, and most of the questions asked in interviews had not been prepared.

A second consideration was ensuring validity in forming the interview questions. Fowler (2002) explains that validity of the measurement process can be improved by asking multiple questions in different forms that measure the same construct. This helps to average out response idiosyncrasies. Cohen, Manion, and Morrison (2011) suggest that the questions should not be potentially misleading, complex, irritating, or in the negative. The first questions should be of general interest, with attitude questions interspersed throughout. In a study of attitudes of teachers and students towards English language teaching in Japan by Luppescu and Day (1990), it was discovered that the interview questions only garnered successful responses from the teacher participants, and not the students. This suggests that questions need to be designed so they are not unidimensional, requiring metaknowledge about language teaching that students do not have. Luppescu and Day (1990) suggest, "measuring attitudes is no different from measuring other psychological entities such as language ability" (p.131).

On the benefits of conducting interviews in research, Robson (2002) states, "The interview is ...a conversation with a purpose." It is "a flexible and adaptable way of finding things out" (p.228). Certainly the first step in conducting research is to express concisely a specific research problem with commensurate research questions (Minichiello, Aroni, Timewell and Alexander, 1995). My study followed a research design described by Strauss and Corbin (1990) in which the problem was general but the questions were more focused and specifically related to the phenomenon. From the general question of what happens in English writing classrooms at a university in Japan, the specific focus on the aspects of critical argument and writer identity allowed for recognition of the phenomenon of Japanese university students constructing writer identities in the process of taking a course in English composition.

A third consideration was of the intellectual and interpersonal demands when conducting in-depth interviews (Kaufman, 1994). In-depth interviewing means building a relationship through social interaction between the interviewer and interviewee. This collaboration is 
how data in in-depth interviews are created, though a "process of dialogue, negotiation, and understanding" (p.128). In-depth interviews utilize mixed methodologies, so data are not just collected but they also emerge through ethnographic analysis. In the development of this relationship it is necessary to establish rapport and trust. To be able to establish rapport and trust, it was important for me to organize my schedule to allow time for review of field notes, transcription of tapes, and preparation of follow-up questions so the participants understood my intent. I needed to discipline myself to regularly relate the data to the research questions; this way the project could grow in its conceptualization (Strauss \& Corbin, 1990), and participants could see that I was actively listening and appropriately responding to their concerns. There is certainly a general sense that informants want to be good interviewees, but they have to be able to trust that the interviewer will not exploit the informants' honesty.

On the validity of interviews, Cohen et al. (2011) describe several important considerations. They suggest that through "convergent validity," interview measures can be compared with measures that have already been proven valid as long as the measures agree. The most practical way to maintain more validity is by minimizing bias. The interviewer needs to be as neutral as possible, avoiding attitude, opinion or expectation. I did this as an active listener, maintaining a natural setting in which I mostly only asked questions, and only minimally offered my own ideas when asked questions by the interviewee. Psychoanalytical issues in bias including transference (emotions of interviewee projected onto the interviewer) and countertransference (vice versa) were avoided as much as possible by resisting showing my own reactions to interviewee responses. Another possible threat to the reliability of the data collected in interviews that is particularly evident in teacher evaluations is teachers giving a "preferred" or ideal response that is not a true reflection of actual teaching practices (Richards, 2003). For this reason, classroom observations were also conducted to provide a further dimension to the actual teaching practices of the participant teachers.

Invalidity of responses in an interview is an important issue usually caused by some bias. This is to say that respondents may make an error in a response to a researcher-biased question that directs them away from their true answer, and their subsequent responses 
then may continue in that researcher-biased direction. In order to minimize bias, questions were designed to reduce the interviewer's own attitude and opinion as much as possible. Additional considerations of bias of the interviewer include misunderstanding of response, as well as respondents' misunderstanding of the question. It was also anticipated that because I am not Japanese, this may also have affected respondents' answers and my understanding of them (see Neuman, 2003).

Therefore, my interviews questions moved from general to specific and I encouraged informants to expand on their responses as much as possible. The focus on the specific areas of critical argument and writer identity was not brought up if the informant did not do so her/himself. The initial data collection questions with students and teachers are presented in Appendix A. At the end of the year, it was concluded that at least one final follow-up interview would be conducted at the start of the following academic year in order to ask the students' specific questions about the texts selected for analysis. The follow-up interview questions were structured as a functional analysis as described by Ellis and Barkhuizen (2005), also presented in Appendix A.

\subsubsection{Text analysis: Appraisal Theory}

Because of the threats to the validity of qualitative research methods such as observations and interviews, I applied a quantitative research method-text analysis-utilizing a combination of Clark and Ivanič's (1997) selves framework, and Appraisal Theory from SFL to form the basic framework for analysis. I will first discuss the validity of text analysis before then briefly outlining how the selves framework and Appraisal Theory framework were used together to form the analytical framework for the analysis of the students' written texts. The outline includes an explanation of the adaptation of the Appraisal framework to allow for an addition of Clark and Ivanič's (1997) possibilities for selfhood, discussed in section 3.4.

\subsubsection{Validity of text analysis}

Since the 1980s text analysis has been increasingly emphasized as a valuable research paradigm for writing processes research (Bereiter \& Scardamalia, 1983; van Wijk \& 
Sanders, 1999). According to Schilperoord (1996, p.175), the validity of text analysis is problematic as the analytical instruments used are of an "undecided nature". He states,

As was demonstrated discussing Matsuhashi's 1981 study on the temporal aspects of writing, the techniques used to assign hierarchical structures to texts relied rather heavily on the intuition of the analyst and on a rather unconstrained appeal to implicit knowledge resources. (Schilperoord, 1996, p.175)

Because results have remained mostly impressionistic, researchers have sought to approach this research paradigm in a more in-depth manner, with more control, and less researcher-intuitive. According to Sanders and Schilperoord (2006), psycholinguistic processes involved in language production can be identified with an appropriate framework for text analysis.

\subsubsection{The selves framework}

The students' texts were first analyzed for the purposes of identifying which of Clark \& Ivaničs (1997) possibilities for selfhood, or selves, were established (see Appendix C for full initial analysis of selves). This was done initially under the simplest possible terms. If students attributed main ideas to outside sources, this was evidence of a discoursal self. If students made claims or evaluated main ideas, this was evidence of an authorial self. If students contextualized their main ideas locally or personally, this was evidence of an autobiographical self. The identification of these selves was then confirmed using the Appraisal framework. Most students showed evidence of a combination of selves.

For working definitions of the Clark \& Ivanič's (1997) selves, I refer to Chandrasoma, Thompson and Pennycook's (2004) concise definitions. They explain:

[T] he first possibility for self-hood [is] the autobiographical self, which refers to the writer's life history and sense of roots. Second, Clark and Ivanič describe what they term the discoursal self, which is the way in which writers represent themselves in their writing through the types of linguistic and discursive forms they adopt. The third category they call self as author, which refers to the extent to which a writer establishes a sense of authority and authorial presence in a text. (pp.176-177)

In the students' texts in this study, the autobiographical self was made apparent when the writer used personal experience to develop the essay. For example, Yui's essay on global warming displayed an autobiographical self through her references to conditions in her 
local context. She described the problems leading to global warming based on her own experience. To reduce global warming by minimizing garbage, she recommended:

Just say you do not need a plastic bag when going grocery shopping and say you do not need chopsticks when buying food at the convenience store. Carry your own shopping bag and your own chopsticks.

Next, the discoursal self was manifested in the students' representations of themselves through the discursive form of attributing the ideas used to develop the essay to other sources. This was a significant feature of the discoursal self described by Ivanič (1994) that occurs when the writer attempts to identify as a member of the academic community. For example, Ai's essay on sign language education in Japan displayed a discoursal self through her attribution to a number of sources. To support her argument in favor of opening more schools for the deaf in Japan, she drew a connection to the success of American Sign Language education in presenting the following quote:

In the previously mentioned article "The Debate over Deaf Education," Burton cites a comment made by Mr. Koo, a deaf student who succeeded in academics. Mr. Koo explains that "ASL (American Sign Language) exposes children to the world's knowledge and it incorporates self-esteem and aspects of deaf culture."

Finally, the students in this study all displayed an authorial self in their texts. This was displayed through expressing their opinions and stating their own claims. For example, in Aya's paper on the issue of the right to wear religious clothing in schools against school uniform policy, she made a number of claims. These claims were often interpretations of information found in sources. For example, after presenting two statements made by Shabina Begum, the girl who's story was used as the central focus for the argument, she completed the paragraph with the following:

By reading these statements, it is quite obvious that she has very strong feeling toward wearing jilbab. As she states that to wear jilbab is "an obligation", wearing jilbab plays vital role in her daily life and also her mind, too. Therefore it cannot be separated from her life itself. In addition, it cannot be discussed only by the field of how characteristic 
of school uniform or how far a student can reflect her own wishes to the school uniform or education.

\subsubsection{The three systems of Appraisal Theory}

The Appraisal framework assists in "analyses of stance as positioning in relation to values and voices in a text" (Hood, 2004, p. 13), and is therefore ideal for analyzing students' language choices in relation to their socio-cultural academic writer identity. The language features to be analyzed in the Appraisal framework are outlined in the three systems of ATTITUDE, ENGAGEMENT and GRADUATION that position the text (Martin, 1997; 2000).

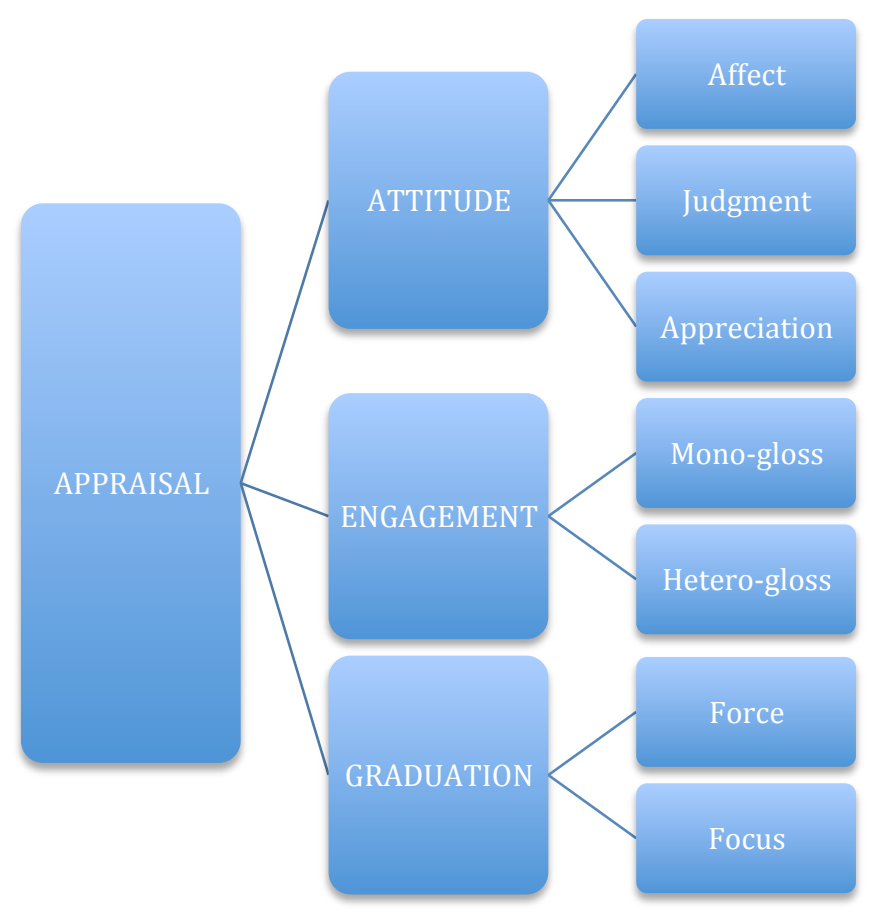

Figure 6: Outline of Appraisal resources in English (as described by Martin, 1997)

This study draws on the Appraisal framework from SFL in order to focus on how the student participants showed evaluation and judgment in their academic writing. The Appraisal framework refers to the interpersonal systems that provide writers with choices as to how they value or "appraise" social experience. The organization for the sub-systems used in the Appraisal framework for this study was based on White's (2005) outline of 
Appraisal Theory, included in the following descriptions of each system. An important adaptation to this framework is the addition of Clark and Ivaničs (1997) "possibilities for self-hood" (p.137) or writer identities described in section 3.4. The idea of a possibility of various selves or writer identities in one's writing is part of what Halliday (1985) referred to as "interpersonal meaning." Halliday explained that language expresses two types of meaning at the same time, namely "ideational meaning" - the topic being communicatedand interpersonal meaning-how the people who are doing the communicating (for this study, the writers) position themselves in relation to the discourse (Fairclough, 1992; Ivanič, 1994). Martin (2000) stresses that an expression of attitude in writing is a truly interpersonal matter, as it is the writer's attempt to establish some solidarity with the reader (Hunston \& Thompson, 2000). Fairclough (1992) made the distinction between the two parts of interpersonal meaning as: 1) the representation of social relations and 2) the representation of social identities. These social identities are constructed in student writers' "discourse choices," which include students' written texts, the fact that the texts were written in the context of an assignment, and the fact that many of the decisions made were unconsciously based on the discourses available to them in their socio-cultural contexts (Ivanič, 1994).

The connection of the selves framework and the Appraisal framework is a decision based on both the literature and evidence. As most appraisal is either inscribed-explicitly stated in the text-or evoked - projected by events or states either prized or frowned on (Hunston \& Thompson, 2000), most appraisal is authorial. The discoursal self is found only in attributing ideas to other sources (located in ENGAGEMENT: Attribution), and the autobiographical self is found only in providing personal response according to the writer's own life experience (located in ATTITUDE).

Regarding the evidence drawing a connection between the selves framework and Appraisal framework, I refer to the student participants in this study. All sixteen students showed evidence of an authorial self in their assertions or claims. Most students showed multiple selves, either by attributing their ideas to other sources (suggesting an authorial-discoursal self), or by referring to local context or personal experience to frame or support their assertions (suggesting an authorial-autobiographical self). 
However, it was noted that this was not always a strict analysis. Some students provided local context in the framing of their argument but did not ultimately rely on that context to develop the thesis (e.g. see analysis of Aya's text in Appendix C), and instead used other rhetorical strategies that I was able to then identify using the Appraisal framework. By combining these two frameworks, I could establish which combination of selves was represented in the students' writing and provide further clarity on what that combination of selves meant in terms of the students' ability to succeed in their writing tasks. By comparing the analysis of selves with the Appraisal analysis, it was noted that all identification of the discoursal self matched all identifications of Attribution, therefore showing a direct link. Making the comparison with the identification of the autobiographical self with the Appraisal analysis, it was noted that all cases matched with the system of ATTITUDE. Details of the analysis are discussed in section 8.3.

The indicators for analysis used with the Appraisal framework and their relationship to the different selves are shown in the expanded figure below: 


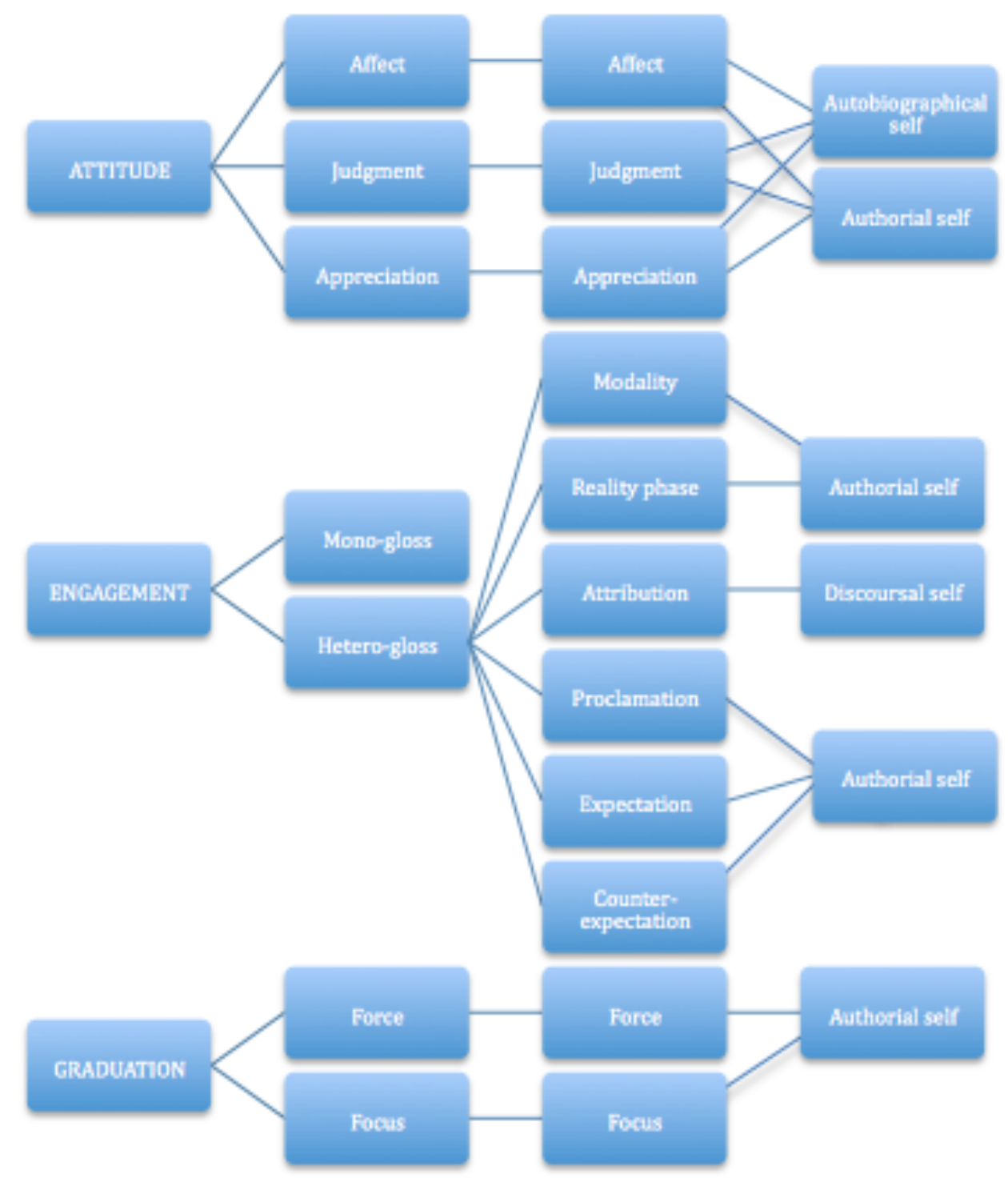

Figure 7: Expanded outline for Appraisal framework for this study, showing indicators and selves

\subsubsection{ATTITUDE: Affect, Judgment, Appreciation}

Within the system of ATTITUDE, there are three sub-systems that comprise a set of language resources for appraising experience: Affect, in terms of emotion; Judgment, in terms of evaluation of human behavior; and Appreciation, in terms of evaluation of objects or products (Martin, 1997; 2000). This system allows for an analysis of the writer's opinion on 
the topic, and whether or not the writer feels strongly about the topic according to personal or social beliefs-both individual and collective-or according to some personal moral code.

Although Martin (1997; 2000) developed a detailed system for a specific analysis of all three of these sub-systems, in this study they are essentially analyzed in terms of two categories: positive or negative, with a sliding scale of intensity according to the adverb of degree, e.g. almost, nearly, completely, very, extremely. The sub-system of Affect is used to pull out all and any instances of positive or negative emotional response. The sub-system of Judgment uses the positive and negative categories and sliding scale focusing on any language of evaluation of human behavior. The sub-system of Appreciation uses the same positive and negative categories, but for any language of evaluation of objects, products, or any other non-human entities. The system of ATTITUDE is used to indicate the presence of an authorial self or autobiographical self, as described by Clark \& Ivanič (1997). A writer who uses a high number of ATTITUDE phrases typically has done so in taking a strong authoritative stance, displaying an authorial self. Depending on the content of those phrases, in the cases where they are based on personal experience and/or local context, this indicates an autobiographical self, as the focus of such language reflects the writer's own life.

\subsubsection{ENGAGEMENT: mono-gloss and hetero-gloss}

The system of ENGAGEMENT provides writers with language resources to position their own voice while negotiating the interaction of other voices and their opinions on the topic. It is concerned with the language writers use in expressing inter-subjective and ideological positions. Martin (1997) divides the system into two sub-systems, namely mono-gloss, for more definitive positions, and hetero-gloss, for less definitive positions. Monoglossia (mono=one + gloss=language) tends towards a unitary meaning, while heteroglossia expands into many meanings (Bakhtin, 1986).

From Martin's model, I have chosen only to focus on the heteroglossia as described by Bakhtin (1986), because it provides a role for potential audiences and a way for the students' texts to be seen to negotiate meaning-making with those audiences. Also, in 
maintaining consistency with its social constructivist basis, this study interprets meaning making in social, not individualized terms. At the same time, in maintaining consistency with the association of critical thinking to academic writing, the study also focuses on ideaforming content and associated doubts about certain "truths." This is all consistent with systemic functional assumptions about language and language use. This study extends its influence from Bakhtin's (1986) notions of heteroglossia and intertextuality, used to explore the positioning of the writer's voice. Bakhtin's notions assert that all texts are intertextual, and that they do not function without referring and/or responding to other texts, and in varying degrees, incorporating other texts-whether actual texts or at least other voices.

The categories of language resources of ENGAGEMENT that I identify in this study are taken from White's (2005) suggested categories. They include the lexicogrammatical categories of: Modality, which concedes that there are other viewpoints; Reality phases, which provide a less-focused position (often using the word seems); Attribution, including hearsay and projection, which usually refers to other sources; Proclamation, which provides a position of certainty (such as in fact, there is no); Expectation, which refers to social norms (such as should, will); and Counter-expectations (such as surprisingly), which refer to positions against social norms.

In this study, the system of ENGAGEMENT was used to identify what Clark and Ivanič (1997) call a discoursal self. The focus is specifically on the writer's use of attributive language, as this is the language that shows whether or not the student attributed the ideas in the paper to outside sources, the specific indicator used for identifying the discoursal self. The other categories further support an authorial self, as they indicate the writer's negotiation of interpersonal space for their own position.

\subsubsection{GRADUATION: Force and Focus}

The system of GRADUATION allows for evaluations to be scaled or "graded" through interpersonal Force (raising or lowering) and preciseness of Focus (sharpening or softening). In traditional grammar, examples of Force may be labeled as intensifiers or emphasizers, most often identified through adverbs of intensification like really or very. It 
can also be identified in quantifiers such as many or a few. Force allows the analysis to place the value on sliding scales in the positive and negative categories. Focus is found in cases of the uses of vague language like sort of or in a sense, therefore softening focus, but also in cases of sharpening focus with adjectives such as true or pure.

As the system of GRADUATION supports the system of ATTITUDE, it further supports the identification of Clark and Ivaničs (1997) authorial self in the intention to make their authoritative stance a strong one and to increase the interpersonal impact of their language.

\subsubsection{Clarification of capitalization, font size, and italics}

It is necessary to clarify the uses of capitalization and italics for the terms related to Appraisal Theory used in this thesis. When the word appraisal is used in relation to Appraisal Theory, it is capitalized. The three systems of Appraisal Theory, namely ATTITUDE, ENGAGEMENT and GRADUATION are reduced-font, all capitals. Sub-types within those systems, such as Judgment and Appreciation within ATTITUDE are capitalized. Italics are used in this thesis on occasion to show emphasis, but also for every example of students' writing.

\subsubsection{Summary}

The instrumentation and validity of data collection for the study required careful consideration of existing analytical frameworks and the necessary adaptations of them to answer the specific research questions raised at the end of the previous chapter. The table presented at the start of the chapter shows how the research questions were answered through a variety of methods of data collection.

\subsection{Data analysis: analytical frameworks and tools}

Now that the methodological framework of the study has been outlined, this section will provide basic descriptions of the analytical frameworks used for the analysis of the observation data, interview data and text analysis. In both the observation analysis and students' written text analysis, existing frameworks were adapted for the purposes of this study. 


\subsubsection{Observations analytical framework}

The framework used for the analysis of the observation data, used especially to answer research questions 1 and 4, was adapted from Ivanič's (2004, p.225) "Discourses of Writing" framework, along with ideas presented by Hyland (2002a; 2005) about how student writers' identities are dealt with in EFL academic writing. Ivanič's framework was developed in Anglophone countries, and therefore recommended to be "refine[d], revise[d] or develop[ed]" for use with research outside those countries. As existing frameworks for analyzing classroom observation data in EFL settings mostly focused on evaluating teaching effectiveness, an adaptation of Ivanič's framework was the most fruitful approach in establishing a relevant analytical framework for identifying factors of identity and argument in the writing classroom. The six discourses of writing (expanded discussion in section 5.4.2) include: skills, creativity, process, genre, social practices, and sociopolitical (Ivanič, 2004, p.225). These six discourses of writing are described according to five criteria:

1. layer in the comprehensive view of language 4 (e.g. the written text, the mental processes of writing, the writing event, sociopolitical and political context of writing)

2. beliefs about writing (e.g. writing is based on knowledge of sound-symbol relationships, a product of writers' creativity, a set of text types based on social contexts, etc.)

3. beliefs about learning to write (e.g. learning to write comes from writing on topics of interest to the writer, writing in real life contexts for real purposes, understanding why different types of writing are the way they are and considering alternative approaches, etc.)

4. approaches to the teaching of writing (e.g. skills, process, genre, functional, critical literacy, etc.)

\footnotetext{
${ }^{4}$ Ivanič (2004, pp.222-224) describes a multi-layered nature of language where a text, beyond simple linguistic substance, is understood within the cognitive processes of the communicator. This is further layered by the literacy event in which the communication is produced, and further again by the socio-cultural and political context in which the communication occurs.
} 
5. assessment (e.g. accuracy, appropriacy, effectiveness for purpose, social responsibility, etc.)

The descriptions of these criteria help to clarify our understanding of teachers' philosophies and goals of writing courses. Having established the teachers' approaches to their course design (outlined in the next chapter), observation data were then analyzed according to two main indicators of writer identity in argumentation:

A. understanding of audience; and

B. development of the thesis

Under the first indicator of "understanding audience" there are three sub-indicators:

- identification of audience-in the form of peer-reading, some mention of readers of the students' writing, as well as considerations of the message;

- how writers signal readers-including hooks, topic sentences/thesis statements, transition signals, or questions to the reader; and

- consideration of persuading readers - through use of active voice, objective stance, being impersonal/objective (use/avoidance of personal pronouns), use of formal language, being explicit, or being sensitive

Under the second indicator of "developing the thesis" there are also three sub-indicators:

- brainstorming (lessons on brainstorming, brainstorming as an activity, and use of feedback in the process);

- establishing a position/claim (reasons for stance, opinion, refutation, counterargument); personal experience as supporting evidence (distinction made from source evidence, reading, summarizing, paraphrasing); and

- students' acceptance/refusal of materials - how do individual students' assessment of materials affect writing instruction?

Using this framework, data were organized into coded manageable chunks. Each feature within each sub-indicator served as a code. A research assistant and I did coding separately in order to compare and confirm the codes. We each did the coding first manually then input our codes into the computer-assisted qualitative data analysis software program 
NVivo. NVivo has been tested and tried for accuracy and reliability and has been shown to be most reliable when used in addition to, not in place of, manual coding (Welsh, 2002).

\subsubsection{Text analysis analytical framework}

For the analysis of the students' written texts, the base analytical framework is the Appraisal framework from SFL as described by Martin (1997; 2000). This framework provides a valuable way to understand students' language choices. In order to gain clearer insight into the phenomena of establishing writer identity and developing critical argument, two additional sources were used to build on the Appraisal framework: Clark and Ivaničs (1997) possibilities for selfhood and Casanave's (2002) writing game strategies. Interview data were used as supporting data for the observation data and students' text analysis and therefore not independently analyzed, except in the cases of research questions 2 and 6.

The features of analysis of the students' written texts follow a three level hierarchy of analysis. At the top are ten selected lexical features (as prescribed by White, 2005) found in the three aspects of ATTITUDE, ENGAGEMENT and GRADUATION as defined by Appraisal Theory, and outlined in Figure 7 in section 4.3.3.2. In the second level in the hierarchy are the possibilities for selfhood. Students display a particular self or selves according to their language choices. Finally the third level includes the writing game strategies, only realized after having evaluated the first two levels of the hierarchy.

To answer research questions 3 and 5, the following features were used in the analysis of students' texts: 1) Self - discoursal, authorial, or autobiographical (Clark \& Ivanič, 1997); 2) Judgment - value of statement made (positive or negative) regarding human behavior; 3 ) Appreciation - value of statement made (positive or negative) regarding processes, products or objects; 4) Affect - emotional/ affectual response; 5) Modality - modals (can, could, may, might, etc.); 6) Reality phase - it seems, it can be concluded, etc.; 7) Attribution credit given to source or hearsay; 8) Proclamation - In fact, It is true, etc.; 9) Expectation of course, etc.; 10) Counter-expectation - surprisingly, etc.; 11) Force (GRADUATION) slightly, very, surely, obviously, etc.; 12) Focus (GRADUATION) effectively, truly, etc.; and 13) writing game strategies used (Casanave, 2002). 
In terms of feature 1, regarding the writers' positioning of themselves, an examination of which of Clark \& Ivanič's (1997) selves the writer chose to represent themselves was used in the analysis, as described in section 3.4. The analysis is based on identifying the subjectpositions of the writer identity, or the various possibilities for selfhood.

For features 2 - 11, as prescribed by Appraisal Theory, which I introduced and discussed in section 4.3.3.1 and which forms the basic analytical framework for the text analysis, the following indicator groups were applied, with sub-indicators serving as independent features, outlined below:

a) ATTITUDE is the values through which a writer expresses affect (valuing of emotions or feelings), and judgment (valuing of others' actions). It includes feature 2 Judgment and feature 3Emotion.

b) ENGAGEMENT is the use of means for positioning the writer's voice with respect to the various intentions expressed by a text. Includes feature 4 Modality, 5 Reality phases, 6 Attribution, 7 Proclamation, 8 Expectation, and 9 Counter-expectation.

c) GRADUATION is the values through which a writer intensifies (feature 10 Force) and shapes (feature 11 Focus) the content of their text.

Finally, feature 12 allowed me to identify culturally determined elements of students' writing through an analysis of their writing game strategies. The arguments used were analyzed for elements considered typical of native English writers, as opposed to those typical of Japanese English writers. The main distinction made was based on how much the student writer "played the game," seen as a Western quality of academic writing (Casanave, 2002).

As was done for the analysis of the observation data, both a research assistant and I analyzed the texts separately in order to compare and confirm the analyses. We discovered no discrepancies between analyses, indicating that the Appraisal framework as it was set up for this study revealed sets of data that could be consistently identified by more than one analyst. The framework allowed me to successfully avoid impressionistic analysis described by Schilperoord (1996), discussed in section 4.3.3.1 above. 


\subsubsection{Summary and use of interview data}

The analyses of the observations and students' written texts will be provided in the subsequent chapters. The data analyses of the observations and students' written texts serve to answer research questions 1, 3, 4 and 5, with supporting data from the interviews. For question 2-What are teachers' goals for these writing courses?-course syllabuses were collected and interview data from the teachers were used to answer the question. For research question 6-Do students recognize critical argument and writer identity as important to their advanced writing education?-only interview data collected from the students could be used to answer the question. All six research questions are answered directly in chapter 9, following the chapters on observation data analysis and students' written text analysis.

\subsection{Ethical considerations}

A brief description is provided in this section of the underlying principles of the ethical considerations of this study. Development in the area of educational research has more recently been concerned with a growing awareness of and increasing emphasis on ethical issues that researchers must address. This is necessary to protect and respect all participants (Cohen et al., 2011; Neuman, 2003; Robson, 2002). This section offers a description of the ethical considerations of my study based on the literature. The following ethical concerns are addressed: (1) informed consent and confidentiality, (2) anonymity of participants, and (3) permission to conduct the study.

\subsubsection{Informed consent and confidentiality}

Because the current study involved human participants, for ethical reasons it required informed consent (Neuman, 2003; Cohen et al., 2011). In accordance with informed consent all participants understood that their participation in the study was voluntary, and that information would be kept confidential (Casanave, 2010). Furthermore it is the policy of Victoria University's Human Ethics Committee that all participants understood that they could withdraw from the research at any time. Therefore as an ethical procedure, all participants gave consent for the study by reading the participant information statement and signing the participant information form approved by Victoria University's Human Ethics Committee. In compliance with ethical research, the form provided a statement of 
the nature of the study and a description of the participants' role. See Appendix B for participant information statements and consent form.

\subsubsection{Anonymity}

Anonymity, defined as "the ethical protection that the people who are studied remain nameless" and unidentifiable (Neuman, 2003; p.504), is a further ethical concern. Cohen et al. (2011) assert that anonymity must be assured by all research in advance unless participants express a desire to be identified. For case study research and the importance of context in research such as mine, Robson (2002, p.501) argues that anonymity is more problematic:

Obviously, it is possible to use pseudonyms for persons and settings, but this may not guarantee anonymity (particularly internally), while further changes that you make to seek disguise may distance your report from the reality it is trying to describe or understand. The basic stance usually adopted is to take reasonable precautions to ensure anonymity, and then publish.

On case study research, Casanave (2010) further supports this position emphasizing that researchers should only include essential private information that does not unnecessarily risk revealing the identity of the participants. I therefore chose to use pseudonyms for both the university and participants, and excluded any non-essential information about the university and participants in their description and in the analysis of the data as an extra precaution to ensure anonymity.

\subsubsection{Permission}

Permission to conduct the study was also an ethical consideration, which Cohen et al. (2007, p. 254) define as "access to the organization or institution where the research is to be conducted, and acceptance by those whose permission one needs before embarking on this task". In accordance with the Victoria University of Wellington policy, I consulted with all relevant authorities and necessary permission was obtained from the Human Ethics Committee before embarking on research. Forms pertaining to permission to conduct research are provided in Appendix B. 
Data collected were made available to the participants in the case studies to look at and talk about in case of protest, of which there was none. The descriptions of students' written texts were negotiated with those students so they could challenge my perceptions. The accounts of classroom interaction and interviews were negotiated with the participants so that they could further explain anything that may have been misunderstood.

\subsubsection{Summary}

I accept all responsibility for maintaining confidentiality. As prescribed by Victoria University's ethics application I have made every attempt to keep participants' identities secret as much as possible. All data collected is in a safe and secure location. All participants have read and signed consent forms allowing me to conduct the research and giving me the right to report my work. The principles of the procedure were strictly adhered to and consent forms signed and collected before research was conducted (Robson, 2002).

\subsection{Chapter summary}

This chapter has provided a description and explanation of the methodological tools used to answer the research questions. Classroom observation data were analyzed using an adapted framework based on Ivanič's (2004) "Discourses of Writing" framework, and were used to answer research questions 1 and 4. Students' written texts were analyzed using Martin's (1997) Appraisal framework from SFL as a basis, with additional features taken from Clark and Ivanič's (1997) concept of possibilities for selfhood, and Casanave's (2002) writing game strategies. The analysis of the students' written texts was used to answer research questions 3 and 5 . The interview data were not independently analyzed but instead used as supporting data for the analyses of the classroom observations and students' written texts. Additionally, the interview data were used to answer research questions 2 and 6.

In the following chapter I will provide a full description of the setting for the study and the participants. Three chapters presenting the analysis of the data will follow; chapters 6 and 7 provide a detailed analysis of the observation data, followed by a detailed analysis of the students' written texts in chapter 8. 


\section{Chapter 5. Who, What, Where: Setting and participants}

Through developing a professional and friendly rapport with academic faculty of English at a university in Japan, I used the approach of stratified purposeful sampling to select participants for the study from a single department at that university. In this chapter I will provide a description of the setting, an explanation of the theory and process of the participant selection, and a brief description of each of the participants in the study. This will be followed by a description of the courses and the teachers' ideas about designing them, focusing on the course objectives and goals as well as materials and assignments. The description of the courses is offered in order to provide a context for the analysis of the observation data in the proceeding chapters.

\subsection{Setting}

I based the study in the English language department at Midori University 5 in Japan. The English department had a student body of about 600 and about 40 full time academic staff-a relatively even balance of Japanese and Western teachers. Students were required to take certain "core" courses that focused on language skills such as reading, writing and speaking (presenting). The Eisakubun or English composition courses (English Composition 1 and English Composition 2) were compulsory. The teachers of the compulsory courses were mostly Western, native-speaking teachers of English, some new, and some who had been teaching their English composition courses for more than two decades. There were also some newer native-Japanese faculty members with a very high level of English language proficiency teaching the compulsory courses. Students were placed in their classes according to the result of a listening test-the philosophy being that students with a higher ability of listening in English would be able to follow lectures that were less altered for easier comprehension.

At the time of the study, no courses in the English department at Midori were required to adhere to any prescribed curriculum, objectives or goals. All teachers were given full

\footnotetext{
${ }^{5}$ For the sake of anonymity, the university selected for the study has been given the pseudonym Midori University. In the English department, all students were English majors.
} 
autonomy to design their classes in any way they liked. There was no sharing of results of the classes, and there were no exit tests. Students who failed these courses mostly did so due to poor attendance, a situation typical of students of English in Japanese universities (Poole, 2005). Also typical of Japanese universities, the students at Midori enrolled in as many as 15 courses in their first year, restricting teachers from assigning extended homework tasks.

Another significant consideration of the setting of this study was the particular struggles younger teachers in the English department at Midori faced in terms of making changes to the system as the older professors had established it. The teacher participants described department meetings in which suggestions for changes such as standardizing course curricula were met with opposition in the form of yelling and pounding the table. The older professors did not want to make any changes to their courses nor did they want to discuss the content of their courses. The teachers (at the level of associate professor or lecturer) would not publish accounts of this inflexibility of older professors, as it would obviously be against their better interest if they planned on carving out a career in the department. However, Jane Barnes Mack-Cozzo (2002), mentioned in section 2.2.2, who wrote about her experience teaching for twelve years at a university in Japan after permanently relocating outside the country, described inflexibility of some Japanese professors and criticized fundamental problems in Japanese culture restricting academic development and advancement as she experienced it. Although this is not an academic source, it does offer a popular view that is otherwise not often publicized. It is important to consider such potential cultural and societal obstacles that the teachers in this study faced in their work environment, where it seems their peers did not welcome innovation in curriculum design.

\subsection{The Selection of Participants}

First it is necessary to provide a description of the theoretical basis underlying the selection of the participants. I begin by explaining the decision to use embedded case studies. This is followed by a discussion of grounded theory and how it applied to the sampling procedure used for the selection of participants. 


\subsubsection{Why embedded case studies?}

This study requires a detailed descriptive examination of the attitudes, experiences and approaches to English L2 writing of students in a Japanese university. The aim of the study is to yield rich, qualitative data that reflects a real-life setting, rather than a less detailed examination of the experiences of a larger number of participants. For this reason, the study is conducted within the theoretical framework of multi-method embedded case studies (Yin, 2009), examining students of English in situ-in English composition classes at a university in Japan. Traditionally, case study research has been met with skepticism by some researchers who argue that the results yielded by such a study are too specific to be of use by other researchers. Yin (1984), for example, argued that case studies should be subject to the same measures of validity and reliability as more empirical studies. In more recent times, however, such claims are held less strongly and the case study is viewed as a legitimate form of inquiry (see Yin, 2009; Cohen et al., 2011; Edge \& Richards, 1998; Nunan, 2003 for discussion). Eisner (2003) provides insight into why this change in perspective has taken place:

One reason for change is that scholars have become attracted to the idea of getting close to practice, to getting a first-hand sense of what actually goes on in classrooms, schools, hospitals and communities. That kind of knowledge takes time. The oneshot commando raid as a way to get the data and get out no longer seems attractive. You need to be there. A clean research design with tight experimental controls might be right for some kinds of research, but not for all kinds. (p.54)

This view is supported by Cohen et al. (2007) who state that the strength of case studies is that they "penetrate situations in ways that are not always susceptible to numerical analysis" (p.181). In regard to criticisms of a lack of generalizability of case study research, Richards (2003) argues that in the field of TESOL "the power of the particular case study to resonate across cultures should not be underestimated" (p.21). Case studies can be generalized to theory, not to populations. For my study, the population is university students with considerably high English language proficiency learning English writing in Japan. I have no intention of making any generalizations about such a population. Instead, it will be an analytic generalization proffering theory that can be extended in further studies. 
With its theory-building structure, this study is both explanatory and exploratory. The theory generated by this study covers broader theoretical issues surrounding learning English academic writing and the role of Western concepts of critical argument and writer identity in undergraduate studies in a Japanese university. These two concepts represent the building of theory in English academic writing (Benesch, 2001; Canagarajah, 2002). It can be seen, therefore, that the case study is a powerful tool that will provide for a firsthand sense of the processes taking place in English writing classes at Japanese universities.

Another advantage of answering the research questions through case study research is that the case study is, methodologically, a "hybrid" in that it utilizes a range of methods for collecting data, rather than being a single procedure (Nunan, 2000). By conducting the research as a case study, an opportunity is created to elicit qualitative data through use of a wide range of research instruments such as questionnaires, interviews, classroom observations and document analysis-as outlined in the previous chapter. The case study design, therefore, allows the use of this large number of data collection methods, which will further improve the accuracy and richness of the data collected in the study.

\subsubsection{Sampling procedure}

Because my research is based on grounded theory in which data are collected first through a variety of methods (described in the previous chapter) and theory is generated from them, and as my study is exploratory and inductive focusing on a small number of cases, I decided to implement stratified purposeful sampling (Llewellyn, Sullivan \& Minichiello, 1999; Patton, 2002) for the selection of participants. A stratified sample reproduces a population in a more manageable size. The population this study deals with is Japanese university students taking English writing classes at a university in Japan. Therefore the student participants had to be Japanese, and in the same writing classes as the teacher participants. I limited the number of student participants to 16, which according to Miles and Huberman (1994), was small enough to allow a rich examination of each case but not too "unwieldy a number of participants for a single researcher to be able to treat the study with high complexity" (p. 30). Moreover, through the examination of a small number of 
similar cases, it served to strengthen the precision, validity and stability of findings (Yin, 2002).

Stratified purposeful sampling is described by Patton (2002) as not meant to produce results for generalization. The idea is that purposeful samples can be arranged or classified by selecting particular cases that provide desired variation; in the case of this study that was decided by their time spent studying immersed in English abroad. Stratified purposeful sampling was valuable for my study because enough information was known to identify the characteristics that influence how the phenomenon (i.e. Japanese university students learning EFL writing) occurs.

The selection of the university was based on my access to it. I chose to conduct the study in the English department as English composition there was compulsory for two years, and the students often studied abroad or went on to post-graduate studies in English. I selected four teachers who allowed me to observe their English composition classes over a period of one year (the full length of the course) and interview them regularly along with two to four of their students. The selection of the teacher participants was primarily based on scheduling and availability, but the variety of pedagogical approaches was also a factorsome grammar-based, others communicative. Also, teachers were selected based on their backgrounds-two were Japanese and two were native English speakers. Student participants in the English composition classes taught by those teachers had volunteered for the study. Based on initial interviews it was discovered that they fit into three categories of educational backgrounds: some had never studied abroad, some had studied abroad for just one year, and some had studied abroad for 5-7 years. As it worked out, essentially all student participants who volunteered to participate were selected; however three students were not selected, as their classes ( $E$ and F) were overrepresented. In total there were six classes and sixteen students.

\subsection{Description of participants}

The description of the participants is as follows: teachers have been given pseudonyms representing the class groups, and students have been given pseudonyms for the purposes of maintaining anonymity. 
Table 2: Teacher and student participants by course

\begin{tabular}{|l|l|l|}
\hline Teacher & Composition classes taught & Student participants \\
\hline Ms. Aiba & $\begin{array}{l}\text { two sections of English Composition } 2 \\
\text { (classes A and B) }\end{array}$ & $\begin{array}{l}\text { class A: Fumiko and Megumi } \\
\text { class B: Miki and Hiromi }\end{array}$ \\
\hline Mr. Clark & $\begin{array}{l}\text { several sections of English Composition 1 } \\
\text { (only his “class C" was used in the study) }\end{array}$ & class C: Yui, Saki, and Aki \\
\hline Mr. Doi & $\begin{array}{l}\text { one section of English Composition 2 } \\
\text { (class D) }\end{array}$ & class D: Aya and Satoko \\
\hline Ms. Ellis & $\begin{array}{l}\text { one section of English Composition } 1 \\
\text { (class E) and one section of English } \\
\text { Composition 1 (class F) }\end{array}$ & $\begin{array}{l}\text { class E: Nana, Yuki, and Ai } \\
\text { class F: Rika, Akiko, Hideo, } \\
\text { and Chinami }\end{array}$ \\
\hline
\end{tabular}

\subsubsection{Teacher participants}

The teacher participants were mostly relatively younger faculty members. The two nativeJapanese teachers were both permanent while the two native-English teachers were shortterm contract lecturers. Ms. Aiba and Ms. Ellis had taught EFL composition in the department prior; Ms. Aiba had extensive teacher training before coming to Midori, while Ms. Ellis had been a student before coming to Midori with no full time teaching experience. Mr. Clark had taught EFL composition for many years in other university departments in Japan, and Mr. Doi was teaching English composition for the first time, though with a strong background in university teaching of content-related courses. All teacher participants were assigned their English composition classes upon starting at Midori. The following table shows the key characteristics of the teacher participants: 
Table 3: Key characteristics of teacher participants

\begin{tabular}{|l|l|l|l|}
\hline Teacher & L1, position & EFL Training & Experience \\
\hline Ms. Aiba & $\begin{array}{l}\text { Native Japanese, } \\
\text { permanent }\end{array}$ & $\begin{array}{l}\text { Extensive postgraduate } \\
\text { level language teacher } \\
\text { training in N. America }\end{array}$ & $\begin{array}{l}\text { 5 years university language } \\
\text { teaching outside Japan, } \\
\text { year teaching at Midori }\end{array}$ \\
\hline Mr. Clark & $\begin{array}{l}\text { Native English, } \\
\text { contract }\end{array}$ & $\begin{array}{l}\text { Postgraduate studies in } \\
\text { TESOL and foreign } \\
\text { language acquisition }\end{array}$ & $\begin{array}{l}\text { 10+ years of TESOL } \\
\text { (university and other) in } \\
\text { Japan }\end{array}$ \\
\hline Mr. Doi & $\begin{array}{l}\text { Native Japanese, } \\
\text { permanent }\end{array}$ & $\begin{array}{l}\text { None (postgraduate } \\
\text { studies in N. America) }\end{array}$ & $\begin{array}{l}\text { 10+ years of non TESOL } \\
\text { university teaching in Japan }\end{array}$ \\
\hline Ms. Ellis & $\begin{array}{l}\text { Native English, } \\
\text { contract }\end{array}$ & None & 2 years teaching at Midori \\
\hline
\end{tabular}

\subsubsection{Student participants}

The student participants had a range of backgrounds, some having been educated in English for portions of their lives overseas or in international schools in Japan. This was typical of the student body in Midori's English department. In initial interviews it was noted that many students had plans to study abroad (in English) and/or go on to postgraduate studies either in Japan or overseas. As of 2011, of the sixteen students, nine participated in study abroad programs either in the US or Australia, and four went on to postgraduate studies, three in the US and one in English in Japan.

A brief description of all sixteen student participants follows. The first group is made up of the six students with traditional backgrounds in Japanese schooling with little or no experience overseas. The second group of four students had spent about one year abroad in English-speaking schools, in addition to their background in local Japanese schools. However, one student entered an international school on her return to Japan after her year abroad. The third group of six students all spent anywhere from five to seven years in overseas studies in English and returned to Japan to attend either international school, schools that had programs designed for "returnee" students, or schools that placed special emphasis on English education. 


\subsubsection{Group One: Traditional Japanese schooling}

This group consisted of six students who had traditional Japanese schooling with little or no overseas experience. Their English language education for the most part started in grade 7 and went for six years, until their graduation from high school. Their English classes were mostly taught by native Japanese teachers, with writing experiences heavy on grammar-translation, and very little writing in English beyond the sentence level.

Aki. C class (1 ${ }^{\text {st }}$ year). Although Aki had the usual limited writing experiences in English, she did write many essays in Japanese. She went to a private Catholic high school and had communication classes in English once a week. She hoped to improve her writing all around, in order to make it more "comfortable" for her readers.

Saki. C class (1 $1^{\text {st }}$ year). Saki reported that her high school was well known for its English language studies, though she also reported very little experience with extended writing in English. Her interest in English came from a desire to travel, and she planned to study abroad in her $3^{\text {rd }}$ year in the US.

Ai. E class (2 ${ }^{\text {nd }}$ year). Before coming to university Ai had taught herself to write essays for exam purposes. Before her first year university writing course which she felt very strongly taught her all her academic writing skills, she had only learned grammar translation. She wanted to improve her academic writing as she had hoped to study abroad.

Akiko. F class ( $1^{\text {st }}$ year). Akiko reported that she had never taken a writing class in English before coming to university, but that she liked English. She hoped to develop her writing skills for study abroad in her $3^{\text {rd }}$ year in the US, and for future postgraduate studies in English.

Hideo. F class ( $1^{\text {st }}$ year). Though Hideo had English classes for six years in his local school, he also chose to study English in an outside language school for three years in junior high school. He reported that he had never taken a writing class in English, and had very limited experience writing in Japanese. He hoped to improve his English fluency through writing practices. 
Chinami. F class ( $1^{\text {st }}$ year). Chinami had no overseas experience except for a 3-week class trip to Australia in $10^{\text {th }}$ grade. She reported that she learned how to write sentences and paragraphs in English in school, but was not a confident writer. She did self-study to learn how to write an essay for the entrance exam. She felt the university course in which she was enrolled did not offer enough actual writing practice (in class). She aspired to become an interpreter and did not perceive writing as important as other skills in her English education.

\subsubsection{Group Two: Traditional Japanese schooling plus one year overseas}

This group consisted of students who had spent approximately one year overseas immersed in English taking all classes in English. This was in addition to their study in local Japanese schools with similar experiences in English education as those in group one.

Aya. D class (2nd year). Aya spent her $2^{\text {nd }}$ year of high school in England, giving her an interest in further studying English. Although she reported having very limited experience with writing before entering university, she felt she was an intellectually curious student who enjoyed the challenges of writing in English.

Nana. E class (2nd year). Nana spent one year in New Zealand attending public school at the age of 10. She reported that she had not learned academic writing in English until her first year at university. Her first year writing course inspired her to want to improve her academic writing and to study abroad in her $3^{\text {rd }}$ year in Australia.

Yuki. E class (2 ${ }^{\text {nd }}$ year). Yuki spent one year in Dubai attending an international school at the age of 14 . She reported that she had a very limited background in writing. She explained that before her first year at university she had "never learned how to write", even in Dubai when she had to write a research paper. She also said in her first interview that she did not like writing and was not a confident writer, so she really hoped to improve because she planned to study abroad in her $3^{\text {rd }}$ year in Australia.

Hiromi. B class (2 ${ }^{\text {nd }}$ year). Hiromi had a background of what she described as "lessrigorous" international schooling in Japan, with just one year abroad in the US, from the ages of 6 to 7 , and then for one month at the age of 17. She reported that her school did not 
offer much in terms of English education as that was not the focus, and that she had very limited experience with academic writing in English. She transferred to Midori from another university in order to focus on English as she planned to do postgraduate studies in English.

\subsubsection{Group Three: International schooling}

This group consisted of students who had spent five to seven years overseas, and had returned to international schools, schools designed for returnees, or schools with a specialized English language curriculum.

Fumiko. A class ( $2^{\text {nd }}$ year). Fumiko spent five years in China attending international schools from the ages of 13 to 17 , and then finished her schooling at an international school in Japan. She reported that she excelled very quickly in English and had extensive experience with academic writing in English, making her extremely capable, though "not very confident." She was a highly proficient speaker of English with native-like fluency and a keen interest in maintaining her bilingualism. Because she planned to study in the US the following year, she was very motivated to learn and further improve her writing.

Megumi. A class (2nd year). Megumi spent six years in the US, from ages 4 to 10 , but reported that she did not remember doing much writing at that time. She then attended a private school for the remainder of her schooling in Japan that she described as a wellknown place for "returnee" students. She was in classes with only returnee students until age 16, allowing her to continue using her English at least casually with her classmates. She had a fairly high level of English with native-like spoken fluency, but was concerned about her ability in Japanese and was concentrating on improving that. She hoped to improve the academic quality of her writing for fear of becoming semi-lingual, i.e. underdeveloped in any language.

Miki. B class (2 ${ }^{\text {nd }}$ year). Miki spent five years overseas, attending an international school in Holland from ages 7 to 11, then one year of school in the UK. She returned to Japan at the age of 12 and attended a special school for returnee students for the remainder of her schooling, allowing her to continue using English with her classmates. She had a fairly high level of English with native-like spoken fluency, and was confident as a bilingual. Her 
expectations were mixed since her writing class the previous year only focused on grammar-translation, but another class required a source-supported research paper. She hoped to improve her writing as she planned to study abroad the following year in the US.

Yui. C class (1 $1^{\text {st }}$ year). Yui spent seven years in the US, from ages 8 to 14 . When she returned to Japan she enrolled in the "International Baccalaureate" program at a private international school, in which she took courses in English with native speaking teachers, and wrote several papers in English including a 4,000-word research paper. She had a fairly high level of English with native-like spoken fluency, and expressed confidence as a bilingual. Although she had extensive writing experience, she reported having very little confidence in her writing. She hoped to improve her language structure and organization in the class.

Satoko. D class (2nd year). Satoko spent six years in the US, from the ages of 10 to 15 . She then attended a high school that she said had a special focus on English. She had native-like spoken fluency and reported extensive academic writing experience before university but her expectations of the course were low. This changed as she discovered the depth of the tasks presented to her by her teacher. She hoped to improve her writing for study abroad in her $3^{\text {rd }}$ year in the US.

Rika. F class (1 $1^{\text {st }}$ year). Rika lived in England for five years from the ages of 7 to 12 , attending local schools. She reported having good experiences learning to write in English at that time and felt she was a confident writer. When she returned to Japan she attended local Japanese schools that she said emphasized English and she wrote many essays in English in high school with native teachers. She had native-like spoken fluency in English. She had no particular expectations with her writing, and felt the course plan to focus on essay writing rather than grammar was good.

\subsubsection{Summary and table}

In summary, of the sixteen student participants, six had no overseas experience studying in English, and four had one year overseas, three of those returning to local Japanese schools and one to international school. The remaining six lived anywhere from five to seven years overseas and returned to Japan. See details in the following table: 
Table 4: Summary of student participants

\begin{tabular}{|c|c|c|c|}
\hline Group & Name & $\begin{array}{l}\text { Jr/Sr High School in } \\
\text { Japan }\end{array}$ & Motivations \\
\hline \multirow[t]{6}{*}{ Traditional } & Aki (C) & Private & Abroad \\
\hline & Saki (C) & \multirow{5}{*}{$\begin{array}{l}\text { Local schools; } \\
\text { English for six years } \\
\text { from Jr high school }\end{array}$} & Abroad \\
\hline & Ai (E) & & - \\
\hline & Akiko (F) & & Abroad/ Post-grad \\
\hline & Hideo (F) & & Abroad \\
\hline & Chinami (F) & & - \\
\hline \multirow{4}{*}{$\begin{array}{l}\text { Fairly traditional, } \\
\text { with approx. } 1 \text { year } \\
\text { overseas }\end{array}$} & Hiromi (B) & $\begin{array}{l}\text { Less rigorous int'l } \\
\text { school }\end{array}$ & Post-grad \\
\hline & Aya (D) & \multirow[t]{3}{*}{ Local school } & - \\
\hline & Nana (E) & & Abroad \\
\hline & Yuki (E) & & Abroad \\
\hline \multirow{6}{*}{$\begin{array}{l}\text { 5-7 years overseas, } \\
\text { with English } \\
\text { language focus on } \\
\text { return to Japan }\end{array}$} & Fumiko (A) & \multirow[t]{2}{*}{ Int'l school } & Abroad/ Post-grad \\
\hline & Yui (C) & & - \\
\hline & Megumi (A) & \multirow[t]{2}{*}{$\begin{array}{l}\text { Returnee private } \\
\text { school }\end{array}$} & - \\
\hline & Miki (B) & & Abroad/ Post-grad \\
\hline & Satoko (D) & \multirow[t]{2}{*}{$\begin{array}{l}\text { Local school English } \\
\text { focus }\end{array}$} & Abroad \\
\hline & Rika (F) & & - \\
\hline
\end{tabular}

\subsection{The courses}

At this stage, an overview of the observed courses is important in order to establish a context for the data analysis and answer to research question 2: What are teachers' goals for these writing courses? This section will first provide a description of the courses as designed by the teachers with a focus on the teachers' intent in establishing course 
objectives and goals and choosing course materials and writing assignments. This description is followed by an identification of which of Ivanič's (2004) Discourses of Writing approaches (discussed in section 4.4.1) the teachers were taking in their courses.

\subsubsection{The teachers and their courses}

The teachers and courses observed in this study could be grouped together in a myriad of ways: by native speaker, tenured or non-native speaker, contract teachers; or by teachers with language education backgrounds and those without. In any of these divisions there are two teachers in each group, which though balanced, does not offer a useful perspective on which to build the analysis.

Therefore, it will serve better to provide a description of each teacher and her/his class(es) as a case. Included in this description is: teacher identity, educational background and teaching experience; course and student proficiency level, objectives and goals; and course materials and assignments. The following table shows these features in an expanded version of Table 1 found in section 5.3.1 on the key characteristics of the teachers. Although the course information provided course objectives and goals as well as materials and assignments, this was all confirmed in initial teacher interviews. 


\begin{tabular}{|c|c|c|c|}
\hline $\begin{array}{l}\text { Course/ } \\
\text { Teacher }\end{array}$ & $\begin{array}{l}\text { Identity, educational } \\
\text { background, \& teaching } \\
\text { experience }\end{array}$ & $\begin{array}{l}\text { Course level, objectives } \\
\& \text { goals }\end{array}$ & $\begin{array}{l}\text { Course materials \& } \\
\text { assignments }\end{array}$ \\
\hline $\begin{array}{l}\text { A\&B/ } \\
\text { Ms. } \\
\text { Aiba }\end{array}$ & $\begin{array}{l}\text { Native Japanese, } \\
\text { postgraduate level } \\
\text { language teacher training } \\
\text { in N. America, } 5 \text { years } \\
\text { university language } \\
\text { teaching outside Japan, } 1 \\
\text { year teaching at Midori }\end{array}$ & $\begin{array}{l}\text { Composition } 2 \text { highest } \\
\text { proficiency level, } \\
\text { practical skills, students } \\
\text { should be able to write } \\
\text { for their real near future } \\
\text { needs }\end{array}$ & $\begin{array}{l}\text { Sem 1: grammar } \\
\text { textbook, grammar } \\
\text { exercises; Sem 2: Letter } \\
\text { to the editor, book } \\
\text { review, MBA application } \\
\text { essay, promotional } \\
\text { webpage }\end{array}$ \\
\hline $\begin{array}{l}\text { C/ Mr. } \\
\text { Clark }\end{array}$ & $\begin{array}{l}\text { Native English, } \\
\text { postgraduate studies in } \\
\text { TESOL and foreign } \\
\text { language acquisition, } 10+ \\
\text { years of TESOL (university } \\
\text { and other) in Japan }\end{array}$ & $\begin{array}{l}\text { Composition } 1 \text { high } \\
\text { proficiency level, basic } \\
\text { academic skills, students } \\
\text { should be able to form } \\
\text { basic essays }\end{array}$ & $\begin{array}{l}\text { Sem 1: grammar } \\
\text { workbook, writing } \\
\text { process, paragraphs; } \\
\text { Sem 2: Six different } \\
\text { essays (process, } \\
\text { compare-contrast, } \\
\text { problem-solution, etc.) }\end{array}$ \\
\hline $\begin{array}{l}\text { D/Mr. } \\
\text { Doi }\end{array}$ & $\begin{array}{l}\text { Native Japanese, } \\
\text { postgraduate studies in N. } \\
\text { America, } 10+\text { years of non } \\
\text { TESOL university teaching } \\
\text { in Japan }\end{array}$ & $\begin{array}{l}\text { Composition } 2 \text { high } \\
\text { proficiency level, basic } \\
\text { academic skills, students } \\
\text { should be able to write } \\
\text { proper paragraphs and } \\
\text { compose a } 2000 \text {-word } \\
\text { essay }\end{array}$ & $\begin{array}{l}\text { Sem 1: paragraphs, } \\
\text { critical reading, } \\
\text { analytical writing; Sem } \\
\text { 2: persuasive research } \\
\text { paper }\end{array}$ \\
\hline \multirow[t]{2}{*}{$\begin{array}{l}\text { E\&F/ } \\
\text { Ms. } \\
\text { Ellis }\end{array}$} & \multirow[t]{2}{*}{$\begin{array}{l}\text { Native English, } \\
\text { postgraduate studies in } \\
\text { Japan, } 2 \text { years teaching at } \\
\text { Midori }\end{array}$} & $\begin{array}{l}\text { Composition } 1 \text { (F Class) } \\
\text { low proficiency level: } \\
\text { academic skills, students } \\
\text { should be able to form } \\
\text { well supported essays } \\
\text { and cite their sources }\end{array}$ & $\begin{array}{l}\text { Sem 1: writing process, } \\
\text { basic essay structure, } \\
\text { citing sources; Sem 2: } \\
\text { Four different essays } \\
\text { (narrative, compare- } \\
\text { contrast, cause-effect, } \\
\text { argument) }\end{array}$ \\
\hline & & $\begin{array}{l}\text { Composition } 2 \text { (E Class) } \\
\text { low proficiency level: } \\
\text { academic skills, students } \\
\text { should be able to take a } \\
\text { position on a topic and } \\
\text { support it with reliable } \\
\text { sources }\end{array}$ & $\begin{array}{l}\text { Sem 1: research skills, } \\
\text { literature review; Sem } \\
\text { 2: persuasive research } \\
\text { paper }\end{array}$ \\
\hline
\end{tabular}

\subsubsection{Ms. Aiba}

Ms. Aiba's background in university-level language education, having taught 5 years of foreign language in a university outside Japan, gave her some fairly clear ideas about objectives and goals for her composition courses at Midori. She distributed a course 
information handout on the first day of classes that included the objectives and goals. Some of the information was in English, while other information was in Japanese. Ms. Aiba explained in her initial interview that the course was designed to help students improve their writing, as it was the understanding that these students at the highest proficiency level already had a certain amount of experience writing in English. In the same interview she rejected the idea of discussing writer identity in her course as she felt it was more important that students focus on learning specifically how to complete more practical writing tasks rather than concerning themselves with the metalanguage involved in composition theory.

In her second interview, Ms. Aiba explained that her expectations of the students were based on her experiences teaching outside Japan:

I know it's very unfair of me to compare them to [native English] students, but having taught in [country] for many years, I can't help but feel that these students are not realizing the future consequences of their actions now. Whereas [native English] students, they do have goals in mind. They want to go to grad school; they want to become doctors, lawyers, what have you. And they know what it takes to become that. These kids, they are just living from day to day. (Ms. Aiba, June 5)

The textbook and assignments were selected based on what Ms. Aiba had deemed to be the students' "real needs", and she attempted to design the courses to encourage the students to start thinking about their futures and the kind of writing they could expect. These real needs included the ability to use accurate English at an advanced level, and write different texts for different purposes, making Hyland's (2008) "text-oriented" approach an appropriate description of Ms. Aiba's course design. These courses were the only ones observed where the students did not write an argumentative essay. The first semester was spent on grammar, using an advanced English grammar textbook. The second semester was spent on producing a variety of texts including a letter to the editor on the news of a Japanese student taken hostage in Iran, a book review on a book chosen by the student, a Harvard MBA application essay, and a promotional webpage for Midori University. The assignment chosen for the text analysis was the letter to the editor as it provided more of a balance of argument and identity compared with the other tasks. 


\subsubsection{Mr. Clark}

Mr. Clark's educational background in TESOL and foreign language acquisition along with his $10+$ years of teaching experience in schools and universities in Japan had given him a clear sense of what the course objectives and goals should be for his composition courses. He did not distribute a course information handout as, in his experience, students tended not to pay attention to it. There were however many original and adapted materials distributed as handouts throughout the year, and students were required to keep them in a binder. Even though Mr. Clark's students were considered to be at a high proficiency level of English, his course was focused on developing students' basic skills of writing, as he believed students needed to work at a reasonable pace where they gradually displayed the ability to write to specific expectations. In his initial interview, Mr. Clark explained that rather than focusing students on writer identity, he focused on academic genres. He suggested that students needed to learn to avoid using the pronoun ' $\mathrm{I}$ ' in order to write according to genre expectations, focusing on the topic rather than themselves as the writer. This did not mean that students were not allowed to use the pronoun 'I', but needed to learn how to use it appropriately.

In the same interview, Mr. Clark explained the design and materials for his courses were based on his experiences teaching writing in Japan:

Jim: How was the curriculum decided for your writing course?

Mr. Clark: I decided it myself. I've been in the fortunate or unfortunate situation of every semester that I've ever taught English in Japan I've always had a writing course. So with all that experience of teaching writing I sort of have an idea of what Japanese learners would probably benefit most from. Deciding it, I've used a lot of different textbooks. And just for my own preferences and my own experience, I haven't really found one that's worked very well. So I end up making a ton of handouts that I give to my students in lieu of an actual textbook. I adapt information from textbooks and I just make worksheets and I make my own, sort of, handouts and here it's about academic writing. I figure that's the best writing that they could learn because it'd be easier for them to go lower and do informal sorts of writing once they get out later. (Mr. Clark, May 10) 
Like Ms. Aiba's courses, Hyland's (2008) "text-oriented" approach also fits the description of Mr. Clark's course. In the first semester, the students completed numerous grammar task handouts created by Mr. Clark. The focus was on accuracy and the ability to make students' writing meet his expectations. They did not write beyond the paragraph level until the second semester when they were required to write six different essays, all with multiple drafts. Each essay required students to complete "fill-in-the-blank" outlines before drafting the essay, and students peer read each other's essays in class. The assignment chosen for the text analysis was the problem-solution essay, although any of the analytical essays could have been used as they all included elements of argument and identity.

\subsubsection{Mr. Doi}

Mr. Doi was the only teacher participant teaching English Composition for the first time. In contrast to Ms. Aiba and Mr. Clark, he had no background in language education beyond his own experiences as a native Japanese speaker writing in English in his postgraduate studies in North America. The course information handout was in Japanese, and included basic objectives and goals. As for the course content, when asked about getting students to focus on their writer identities in his first interview, he confessed, "I haven't thought about it" (May 9). Because Mr. Doi had not taught composition before, he asked a friend with many years experience teaching English composition to suggest a textbook, which he used. It was a bilingual Japanese-English textbook for introductory English composition written by a Japanese academic. It included lessons on the writing process and writing paragraphs. Just before the course started, he decided he needed to offer something more rigorous. He explained the situation in his initial interview:

I asked my friend who had composition class in different university and since I didn't know the level of student, so I assumed that the worst case scenario which is to teach the most basic class, fundamental class, the least, I shouldn't say this word, but least prepared student and therefore I came up with that textbook that I'm using now. Then as soon as I arrived on this campus I was introduced to [teacher] and others who have taught the English composition class. They gave me totally different information than I expected which is to make the class more challenging, more intellectually stimulating, and I showed them the textbook and they said, "Oh this is basic alright", but too late because I have put on the Web saying that all the students who enroll in this compulsory class needs to purchase this text in advance. So I compressed the fourteen lessons into seven and I came up with additional idea 
of how to add some more interesting stuff at the second half of the semester. I didn't have time though. So I frantically looked around and found out there was another textbook prepared by two people at my alma mater. And at the beginning of that textbook it says, "How to actively read and to write." It has three different essays on Barbie doll ... that will bring us to the end of this semester. Then next semester I put TBA, TBA, TBA. I had to keep it pretty open because I didn't know what to expect. (Mr. Doi, May 9)

Mr. Doi explained that in his experience learning to write academic English he felt the most important skill was structure as it was where he experienced the most difficulty in transferring his Japanese writing skills to English. However, he did not know how he could incorporate structure into his course. Mr. Doi felt very much as though he was learning how to teach composition throughout the observed course. This is most likely what led to his course taking more of what Hyland (2008) described as a "writer-oriented" approach, where his position as a non-native speaker of English, learning how to teach English composition in the process of teaching it, became a central part of the course as students in the first semester were required to write a critical essay using the Barbie readings from the textbook, and in the second semester to write a critical, persuasive research paper. The research paper was the assignment chosen for the text analysis, as it was the main assignment for the course.

\subsubsection{Ms. Ellis}

Like Mr. Doi, Ms. Ellis also had no background in language education beyond her experiences as a native English speaker writing in Japanese in her postgraduate studies in Japan. However, she was in her third year of teaching at Midori and had designed her course based on the two years' previous experience using textbooks she ordered online. In her first interview when asked about focusing on writer identity, Ms. Ellis rejected the idea

of discussing it in class as she felt it was more important that students focus on writing tasks without concerning themselves with abstract issues such as writer identity that may confuse them.

For both her Composition 1 and Composition 2 courses, the materials were copies of pages from a number of different textbooks, all from the US. Especially in the Composition 1 course, a textbook on controversial topics was used to provide sample argumentative 
essays and a list of topic suggestions for students to write on. It took considerable adapting of the materials for the low proficiency level students in Ms. Ellis's classes. The result was an academic skills focus that involved students moving quickly through basic writing skills practices on the writing process and paragraph formation to learning how to cite sources and develop convincing argumentative essays in Composition 1, and writing persuasive research papers in Composition 2. It was those argumentative essays from Composition 1 and the research papers from Composition 2 that were chosen for the text analysis.

Ms. Ellis's courses maintained what Hyland (2008) described as a "reader-oriented" approach in which students were challenged to establish a clear, critical position on their topics and support and develop those positions with convincing evidence. While in the Composition 1 course there was a considerable focus on writing forms in the first half of the year displaying a clear "text-oriented" approach, there was less emphasis on forms in the second semester when the focus was on writing convincingly. In Composition 2, the stress was on persuasive writing and successful establishment and defense of a critical position on a research topic, more indicative of Hyland's (2008) "reader-oriented" approach.

Since the writing courses observed in this study were all completely designed by the four teacher participants with no guidelines, requirements or restrictions provided by the university, faculty or department, the teachers made their own decisions about every aspect of the course. Multiple discourses could be found in every course. The six discourses of writing (introduced in section 4.4.1) are defined by Ivanič (2004, p.225) as: 


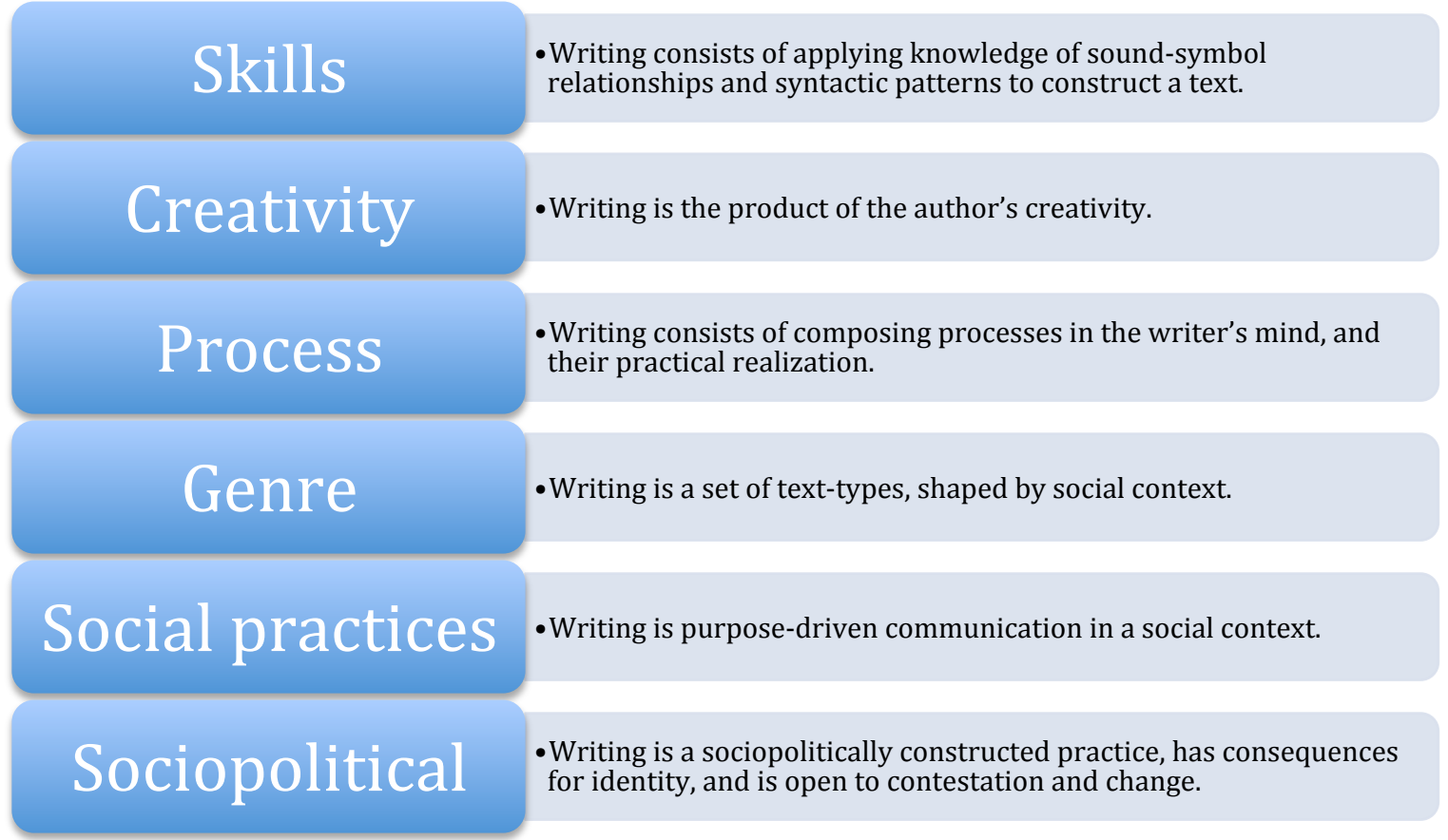

The following table shows the discourses of writing observed in each class throughout the year. This table and the description below provide a context for the answer to research question 3: What are teachers' identifiable cultural expectations of EFL writing? The information presented here is based on the teacher interview data and course documents discussed in section 5.4.1 above.

Table 6: The discourses of writing applied by each teacher

\begin{tabular}{|c|c|c|}
\hline Level & Teacher & Discourses of writing observed \\
\hline \multirow[t]{3}{*}{ Comp 2} & $\begin{array}{l}\text { Ms. Aiba } \\
\text { A/B } \\
\text { classes }\end{array}$ & Skills ( $1^{\text {st }}$ semester); Genre, Social practice (2 ${ }^{\text {nd }}$ semester) \\
\hline & $\begin{array}{l}\text { Mr. Doi } \\
\text { D class }\end{array}$ & $\begin{array}{l}\text { Process, Genre ( } 1^{\text {st }} \text { semester), Creativity, Sociopolitical ( } 2^{\text {nd }} \\
\text { semester) }\end{array}$ \\
\hline & $\begin{array}{l}\text { Ms. Ellis } \\
\text { E class }\end{array}$ & Skills, Process, Creativity, Sociopolitical \\
\hline \multirow[t]{2}{*}{ Comp 1} & $\begin{array}{l}\text { Mr. Clark } \\
\text { C class }\end{array}$ & Skills, Process, Genre \\
\hline & $\begin{array}{l}\text { Ms. Ellis } \\
\text { F class }\end{array}$ & Skills, Process, Genre \\
\hline
\end{tabular}


The relationship between teachers' (NNS $=$ Non-native speaker of English, NS $=$ Native speaker of English) academic backgrounds, teaching orientation, discourse approaches and course goals is represented in the following diagram:

Figure 9: Relationships between significant features of teachers and their courses

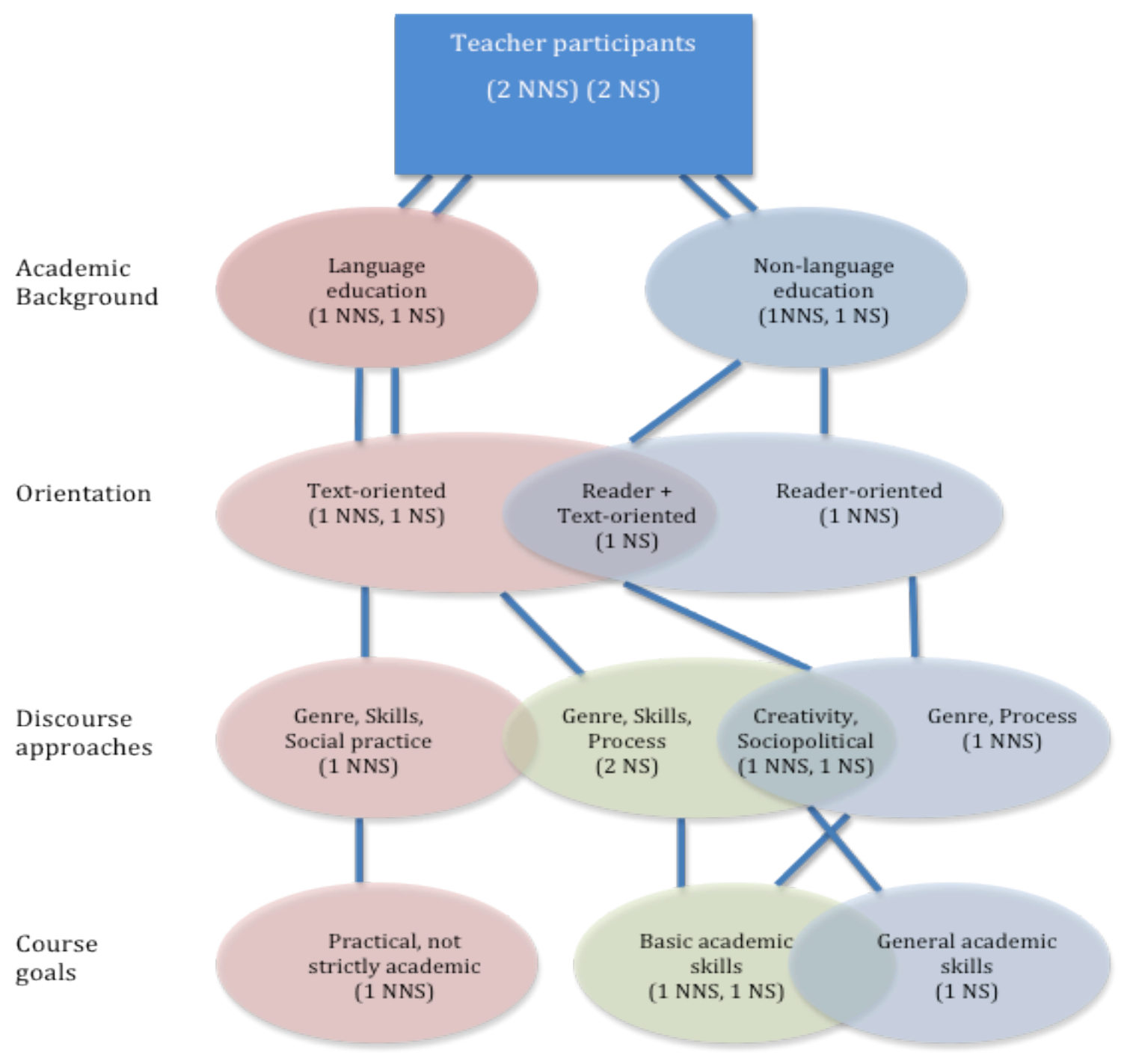


The diagram shows a distinct divide leaving Ms. Aiba as the only teacher to take a social practice discourse approach and set practical writing goals. Mr. Clark who similarly had an academic background in language education and maintained a text-oriented classroom, by adding a process discourse approach ultimately set more basic, academic course goals. This was more in line with the two teachers who did not have academic backgrounds in language education, maintained more reader-oriented classrooms (although Ms. Ellis also incorporated text-orientation) and incorporated creativity and sociopolitical discourse approaches in their attempts to meet academic course goals. I will now provide details about the features of teachers' approaches.

In the Composition 1 classes, Mr. Clark and Ms. Ellis were observed emphasizing a skills discourse of writing. Mr. Clark's students were required to practice a variety of lexicogrammatical patterns and rules before writing extended texts, which were different essays of different genres. Ms. Ellis's students practiced different language patterns for the purposes of sentence variation early in the year. Observations revealed the students in both the $\mathrm{C}$ and $\mathrm{F}$ classes were taken through the steps in the writing process of pre-writing, outlining, drafting, revising and editing for various types of academic essays such as causeeffect, problem-solution, compare-contrast, and argumentative. Where Ms. Ellis's goals differed was indicated by her course outline, where in her fifteen course goals, she included several on argumentation and thinking, including:

- To analyze arguments of others and learn to formulate one's own.

- To realize that a challenge to one's ideas is not a judgment of one's character.

- To know that the point of argument is not to win, but to be able to back up one's position convincingly and persuade others to adopt/acknowledge one's point of view.

- To understand that there are many beliefs, ideas, and opinions in the world, so do not assume to always be right. Instead, give others a chance to help open up one's mind to alternative ways of thinking.

The first of these four points was observed regularly in the F class, in the practice of getting students to write on the board and the class as a whole would offer critiques. However, no particular lessons on the other three points were observed. 
The skills discourse of writing was similarly the main focus for the A and B classes taught by Ms. Aiba for the entire first semester. The classes I observed were consistent: Students studied from a grammar textbook, then presented a different lexicogrammatical point each week. In the second semester, the students in these classes then followed a social practice discourse of writing in which each observed writing task focused on a different genre of writing, some with real-life context such as a letter to the editor, an MBA application essay, and an informational web page. The social practice discourse of writing was unique to Ms. Aiba's classes.

The most varied approaches to the discourses observed in one course were in the D class, which was the only class that did not have any particular skills discourse, i.e. no observed instances of focus on grammar or language patterns. In the first semester, the students were introduced to the writing process and to various genres of writing for the purposes of a major writing task. In the second semester creativity and sociopolitical discourses were added when students selected their own research topics and worked in peer groups to critique and defend each other's and their own thesis.

The multiple discourses of writing observed in the $\mathrm{E}$ class were found at different times throughout the year, though none in any particular depth or defining way in terms of clearly establishing goals of the course. Students practiced with language patterns early in the year. They reviewed the writing process as it had been instructed to them in the previous year. Through creativity and sociopolitical discourses, students were able to choose their own research topics, and worked with peers to some extent in order to discuss their thesis.

These different approaches in the discourses of writing used in the teachers' different classes helped to reveal not only indications of the goals and attempts to reach them, but also the ways the two main indicators of writer identity came to be addressed in the observed classes-the two main indicators being communicative writing (for an audience), and critical thinking (in developing a thesis). The skills discourse, which focused on lexicogrammatical issues, though common in these classes, did not reveal insight into writer identity or critical argument. The process discourse, equally common but in contrast 
to the skills discourse, did introduce ideas about audience and generated discussions between peers, as discussed in section 6.2.1. The genre discourse also revealed important considerations of audience in those classes, as well as considerations of developing the thesis. The creativity and social practices discourses showed that those teachers believed students should learn by writing on topics that were relevant and/or of interest to them, an important indicator of thesis development discussed in the following chapter. Finally, the sociopolitical discourse most directly addresses issues of writer identity and critical argument in its beliefs about writing and learning to write. The sociopolitical discourse served to provide the most insight into the two significant indicators of 1) the writer's understanding of audience, outlined in the following chapter, and 2) development of the thesis, discussed in chapter 7 .

\subsection{Chapter summary}

The setting and participants for the study were purposefully selected according to careful consideration of theory. As an exploratory, inductive qualitative study, it was conducive to utilizing embedded case studies. The university selected for the study was ideal in that the students in the English department often participated in study abroad programs and/or went on to do post-graduate studies in English. The teacher participants provided a variety of backgrounds and pedagogical approaches, and the student participants provided a variety of backgrounds and motivations for participating in the study. The course objectives and goals, as well as course materials and assignments have been outlined in order to provide context for the data analysis.

Furthermore, Ivaničs (2004) Discourses of Writing framework allowed me to identify that of the six possible discourse approaches, all were used by the teachers in this study, but in a variety of combinations of which no two courses (other than A and B) were the same. This understanding of the teacher's approaches to their courses allows for the differences in the classes to be used as a context for the analysis of the observation data. The next two chapters will provide an analysis of the data collected through observations, with supporting data from the teacher and student interviews. 


\section{Chapter 6. Writing to be read: Observations, the discourses and readers}

This is the first of two chapters to examine the observations of the writing classes in order to attempt to answer the overarching question of the study: What happens in an English writing class at a Japanese university before students go overseas for studies in English? The issue here, as outlined in chapters two and three, is that English writing education in Japan seems to be in a constant state of flux in terms of classroom practices. While efforts have been made to make writing more communicative and to incorporate more critical thinking, many university teachers in Japan are not given any guidelines for their courses, nor are they reviewed by an academic committee, and are therefore left entirely on their own to develop their writing courses in whatever way they deem fit. This leaves many seasoned teachers to maintain older, outdated grammar-translation methods in their writing classes. This is why it is important to conduct more qualitative, exploratory research in these writing classes that includes classroom observations, in order to reveal more information about what is happening in them.

This chapter is divided into five sections. The first section will provide an outline of the chapter. Next, three sections are dedicated to the findings from the classroom observations regarding communicative writing practices (i.e. classroom practices that focused on a reader or audience). This is followed by a chapter summary.

\subsection{Chapter outline}

In this chapter, I will provide an analysis of two different aspects of the observation data, along with supporting interview data. As described in the methodology chapter, Grounded Theory was employed as a systematic method allowing theory to be generated from the data, discussed in section 4.1.2. In building sensitizing concepts for the purposes of assisting the discovery of emerging themes, I read about issues of writer identity as they pertain to writing classroom observations in Ivanič's (2004) Discourses of Writing and Learning to Write. These discourses of writing will be discussed first in this chapter in relation to the observations in order to focus on research question 2: What are teachers' goals for these writing courses? Ivanič's article offers a framework that provides six 
discourses as pedagogical approaches to writing education: skills, creativity, process, genre, social practices and sociopolitical (discussed in 6.2.1). Although the teacher participants in this study utilized a variety of the first five discourses in their approaches to their English composition classes, especially skills and process, it is the sixth-a sociopolitical discourse - that is most significant to my study as it pertains specifically to critical literacy and concerns issues related to establishing writer identity and developing critical argument. These issues will be addressed more directly in the second chapter on the observation data analysis, chapter 7.

Using the issues of writer identity and critical argument as sensitizing concepts, theoretical coding was applied, i.e. fractured concepts were woven into hypotheses that worked together to explain the main concern of the participants (Glaser, 2004). Key points in the data collected were marked and grouped into two main indicators of writer identity: 1) the writer's awareness of audience (Ivanič, 2004, p.238), exploring the relationship between the writer and the reader with the understanding that writing is a social, communicative act; and 2) the writer's development of the thesis, exploring the role of critical thinking processes in establishing a position in academic writing (Ivanič, 2004, pp.238-239). From these two main indicators, and from the theory generated by the data, the following categories were formed in the development of the fundamental theory of the study:

Audience (analyzed in this chapter)

- Identification of audience

- How writers guide readers

$\circ$ Persuading readers

Thesis (analyzed in the next chapter)

○ Brainstorming

- Establishing a position or claim

- Use of personal experience

- Students' acceptance or refusal of course materials and content 
The category of "Audience" (i.e. the writer's understanding of audience) will be analyzed in this chapter using further divisions of the three sub-indicators above that emerged from the data (see Table below).

Table 7: Sub-indicators and further division of the writer's understanding of audience

\begin{tabular}{|c|c|c|}
\hline \multirow[t]{9}{*}{$\begin{array}{l}\text { Writer's } \\
\text { understanding of } \\
\text { audience }\end{array}$} & \multirow{2}{*}{$\begin{array}{l}\text { Identification of } \\
\text { audience } \\
\text { How writers guide } \\
\text { readers }\end{array}$} & $\begin{array}{l}\text { Peer-reading } \\
\text { Acknowledging the reader }\end{array}$ \\
\hline & & $\begin{array}{l}\text { Topic sentences/ thesis statements } \\
\text { Transition signals }\end{array}$ \\
\hline & & Hooks \\
\hline & & Questions to the reader \\
\hline & Persuading readers & Active/ passive voice \\
\hline & & $\begin{array}{l}\text { Objective stance/ use of personal } \\
\text { pronouns }\end{array}$ \\
\hline & & Formal language \\
\hline & & Being explicit \\
\hline & & Being sensitive \\
\hline
\end{tabular}

These further divisions served as the codes for the analysis of the observation and interview data. Thus, the observation data are analyzed and presented according to these notions in this chapter. Additional divisions were also made as they emerged from the data on the category of "Thesis", to be discussed in the next chapter.

Therefore, these two chapters are organized in the following manner:

- The concept of the writer's awareness of audience and its subsequent categories will be analyzed (chapter 6).

- Finally the concept of the writer's development of the thesis and its subsequent categories will be analyzed (chapter 7). 


\subsection{Identification of audience}

The indicator of audience found in the writing classes suggested that there was at least some focus on students' understanding that writing is communicative, i.e. that their texts were to be written for a particular reader or readers. These classes taught students that an important part of the writing process was to consider the reader of each of the writing tasks the students completed. In general, the academic writing classes observed for this study showed great variation regarding consideration of audience or readers. There seemed to be two distinct categories of teachers' instruction of communicative writing; the first included all and any discussions of peer reading, and the second included those observed instances when "the reader" was included as part of the consideration of planning for a writing task.

\subsubsection{Peer reading}

In general, the greatest instance of consideration of audience was the mention, discussion and/or explanation and implementation of peer reading, which reminded students that the immediate audience for their writing included any of their classmates in the room. A wide variety of explanations of the purpose and value of peer reading were offered, depending on the teacher.

In the Composition $2 \mathrm{E}$ class taught by Ms. Ellis there was a great deal of emphasis on peer reading when students were assigned to write an extended argumentative research paper. After this, the teacher continued to regularly promote the benefits of peer reading and feedback, dedicating large amounts of class time to developing the students' understanding of these aspects as part of the writing process. The students developed their arguments through various related writing tasks and a series of drafts. Students responded positively to these activities in class, as well as in their interviews. Ai from the E class had this to offer: "When I read others' essays, they are all different, so I think there are many methods of writing, so now I need to learn how to write like that." Ai went on to explain that she felt it was important to have exposure to many different styles of writing so that she could find her own style. She explained that she was still working on it: "I can write sentences as teachers teach it. But I want to write it in a more interesting-more attracting sentences when I make my own essay" (Ai, January 25). Ai was making a distinction between her 
writing and her peers' writing, wanting to adopt some of the styles she felt would improve her writing.

In the Composition $2 \mathrm{D}$ class taught by Mr. Doi there was one observed instance of peer reading in the first semester, referred to in the form of making "comments" on or about other students' papers. In the second semester, most classes were dedicated to peer feedback on the students' papers, often completed in 5 - 10 minute blocks. There were dedicated "commentators" for each student's presentation, and the other students in the class made comments on a feedback sheet. In addition to the written comments, the teacher made these comments available on the class intranet. He explained in class:

I'll make it readable on Moodle, so I'll make it public. I'll scan it, I'll put it on Moodle, so group A can read this. So that will help them move forward for their research. So any comment will help them. So while you're listening, please write down your comments, either for individual presenters or general impressions... anything will help them. (Mr. Doi, D Class, Oct 10)

Thus, Mr. Doi not only placed a lot of emphasis on peer reading and feedback throughout the year, but like Ms. Ellis also allowed class time to complete peer feedback activities. There was some discrepancy between the two D Class student participants' feelings toward the peer feedback. I will first describe Aya's situation and introduce her classmate Satoko's situation in order to show the stark contrast in their experiences of peer feedback.

Aya had a particular issue with ownership of her writing, related quite clearly to her regular comments about at first, a lack of familiarity with the topics used in class and later, a lack of familiarity by her peers with the topic she chose for her research paper. Aya felt a divide between her and the other students she considered to be more fluent, and therefore more critical. Aya's big writing task was an argumentative research paper. Aya had chosen to write about the issue of the wearing of a veil by an Islamic student in a British schoolwhich was against school rules-because she felt she could provide a non-Western perspective on the topic. In addition to concern about the ownership of ideas in this paper, she was also concerned about the large amount of peer-evaluation that had been introduced in the class, as this was a practice she was not particularly comfortable or familiar with. She commented: 
It - oh, they're kind of thinking in the way - a way Western people does. So, it kind of has gap between me and them. So this is - it doesn't really help me - like I said before, it's kind of unsolvable problem. (Aya, October 9)

Unfortunately, her classmates seemed unable to attain the same perspective. They insisted it would be better for her to shift her perspective to a more Western one. She commented:

Then because I - or if I was just studying what - with Western methods. It would be really easy, and it would be really helpful. I could just criticize of her wearing a veil. And argue her right because she's in England. Why don't you adjust? You know, I did. (Aya, November 22)

It became clear Aya was not willing to risk being disconnected from her writing. She commented, "It's really - it would be really unfair of me to write in a way they suggest me to write." (Aya, November 22)

In contrast to Aya's feelings about the peer evaluation, her classmate Satoko was very positive about the peer evaluation she received on her argument on the topic of universal health care in the US. Satoko commented:

Well some people commented on like the construction of my outline or like some people said what I wrote in the thesis is not really covered in the body so like you have to follow your thesis. Some people just were like, Go for it. (Satoko, December 11)

All of this seemed to work very well for Satoko, and she was able to decipher useful comments from the simply encouraging ones. However, for Aya, in her final interview, after the course had ended, she commented:

Aya: $\quad$ My group didn't like my paper because they all thought I needed to change my thesis, and when I re-read it, I thought, the whole essay is crap. The structure, the, the use of words, it's kind of ... it's really amazing crappy! Otherwise it was quite good. What I was trying to say, that was quite good, well-analyzed, I thought.

Jim: So, the idea and the content?

Aya: $\quad$ The idea and the content was really good.

Jim: $\quad$ OK. Were you able to make the point that you wanted to make?

Aya: $\quad$ Yeah.

Jim: What did your classmates say? 
Aya: $\quad$ Oh... well the problem was, I was analyzing the court case quite well. Uh, to the point. That was uh...kind of good. But the argument I set at the beginning, "Why do they wear veils?" doesn't really match the analysis. (January 31)

Aya went on to describe how as much as she had resisted the negative comments from her peer readers throughout the process of writing her research paper, she came to appreciate their concerns about her lack of clarity and purpose with her research question when she attempted to develop the paper further. This reflects some of the "resistance to learning" young adult learners like Aya have to overcome, described by Atherton (2011, discussed in section 3.2.1).

From the A class, it was revealed in an interview with Megumi that a lack of peer reading may have led to a certain hindrance to her learning. Early in the first semester of the Composition $2 \mathrm{~A}$ and B classes taught by Ms. Aiba, the students were introduced to peer reading as part of the task of a "writing journal". However, later interviews revealed that this peer reading never happened due to a lack of time. Then at the start of the second semester, another mention of peer reading was observed (A class, October 10) when the teacher asked the students to check each other's outlines and make recommendations for improving them.

These were the only observed mentions of consideration of peer readers found in the A and B classes. Much of this had to do with course content as decided by the professor. Because the students in these classes were more proficient in English, it was decided that for the first semester they would focus on cleaning up language mechanics, including grammar, spelling and punctuation, which typically did not lend itself to considerations of audience. However in the second semester the focus was on authentic uses of writing including a letter to a newspaper editor, a book review, an application essay, and a webpage-all which simply by the nature of the task consider a reader. In their interviews at the end of the year, some students from the A and B classes commented on the lack of peer reading. From the A class, Megumi, who was concerned her writing was too childish, commented:

Well I know like when I read something I know what's professional and what's not. I can read like other people's stuff and feel what's professional, but I don't know... I wish we had a chance to read each other's papers like we did last year so I could 
know different ways to write them, and then maybe I can make my writing more professional. (Megumi, 22 January 2008)

With regard to Composition 1 (C\& F classes) an even greater discrepancy between classes was observed. In the $\mathrm{C}$ class, peer reading was very explicit, as it was the understanding of Mr. Clark that most of his students had not experienced peer reading before. He gave a detailed explanation of the peer reading task as an activity meant to develop students' writing skills, and gave significant time for peer reading in class in the first semester. In the second semester, Mr. Clark particularly focused on the peer reader's duty to "help" the writer through any kind of feedback. In the F class, however, although there was extensive consideration of "the reader", there were no actual mentions or practices of peer reading observed in the data.

Thus, observation data revealed even though the programs of Composition 1 and Composition 2 were carried out under the same title in all observed classes, the way teachers addressed the notion of audience in regard to peer reading varied greatly according to the teacher and student. The responses from the students about this varied as well. In the A (and B) class, which had the least peer reading, Megumi felt it kept her from improving her writing. In the D class, which had the most peer reading, Satoko felt encouraged by the peer feedback, while Aya felt discouraged by it.

\subsubsection{Acknowledging the reader}

In addition to peer reading, the reader was identified in other ways in the classes. In Composition 2, other acknowledgements of the reader included a discussion of reader versus writer responsibility, consideration of the impact of certain language on an imaginary reader, and consideration of native versus non-native readers. In Composition 1 , acknowledgements included the impact of essay organization on the reader, the impact of establishing an argumentative position on the reader, setting up a clear introduction for the reader, emphasis on the effect the writer's interest in the topic has on the reader, and the importance of refutation for the reader.

First, early in the year, Mr. Doi initiated a hefty discussion on reader versus writer responsibility as the focus of a writing task designed to get students to think about taking a 
position on an argumentative topic. The teacher chose the topic of reader versus writer responsibility and contextualized it in a discussion on the difference of responsibility in the Japanese and English written forms. This generated some deep discussions from the students, as illustrated below:

Student: $\quad$ So the question means that if you agree that in English you have the responsibility-the writer has the responsibility, and Japanese are vague, so Japanese don't care about the readers. Um, and it's like that if the writer's not responsible, so I thought that we won't really know what the writer says. So, I thought the writer has the responsibility. If you write a book, he writes a book because he has something to say... to tell it to the readers... I thought that he has actually the responsibility.

Mr. Doi: $\quad$ Excellent. She used the word 'vague'... ...In comparison to English, Japanese is a vague language. It's a linguistic difference. So, even though the writer tries hard, he may not be able to get the message across, because the language itself is vague. (D Class, May 9)

Conversations like this were prevalent during this teacher-initiated discussion, and indicated explicit acknowledgement of the reader in the curriculum. In other classes, considerations of the audience were less explicit, but nonetheless students were made aware of their audience, particularly in terms of reader understanding, interest and reactions.

Across classes, there were many mentions of acknowledging the reader in regard to language use, with a focus on an awareness of the particular audience assisting in language choices and explanation clarity. In addition to language choices, there were several observed occurrences where a teacher emphasized the importance of establishing for the reader a well thought-out position on an argument by considering all the possible positions people might take:

You don't want to start with the weakest one, the one that has very weak support; your reader might—“Huh? This is the first one? It's so weak!" They won't even finish reading your essay. (Mr. Clark, C class, Oct 17)

If you do not show your readers that you have thought about all your possible positions on the subject, then your writing sounds like nothing, nothing more than just propaganda. Okay? There's no...you're just throwing away your opinions, you're throwing out your opinions without being responsible and showing your reader 
that you have actually considered all the other possibilities, and upon consideration of the other possibilities, you've come to the conclusion that the one you chose is the best solution, okay? (Ms. Ellis, F class, June 6)

Moreover, there was observed consideration of the reader in terms of interest in the research topic. Ms. Ellis, for example, emphasized that the introduction of an argumentative essay was focused on the reader, and thus choosing a topic that is of interest to the writer was a way of encouraging the interest of the reader. Thus, a final consideration of the audience was writing to increase the interest of the reader in the research topic.

\subsubsection{Summary}

Ultimately, the various approaches to getting students to focus on audience offered in the different classes suggest significant discrepancies in courses with the same title. In the Composition 2 classes, students were given the task of peer reading in various forms, with varying levels of instruction on doing the task. This led to very different responses from students regarding their impression of peer reading. The discrepancy between two courses titled English Composition 1 on the issue of peer reading is significant as there is clearly some expectation in English Composition 2 classes that students should already know what peer reading is and how to do it.

Also notable was the particular explanation of peer reading offered by the $\mathrm{C}$ class teacher. It was described as a task for helping others. There was no consideration of the task being beneficial for the reader in any way, in contrast to the focus on peer reading in Composition 2 classes. This particular way of focusing on peer reading does not support theories related to the development of writer identity. As a long time specialist in EAP writing in EFL contexts, Claudia Keh explained more than twenty years ago, "learners can gain a greater sense of audience with several readers (i.e. readers other than the teacher). The reader learns more about writing through critically reading others' papers" (Keh, 1990, p.296). What peer readers learn about writing is part of the development of their ability to establish a writer identity that will be understood by other readers as they attempt to understand the identity of the writer of the paper they are reading. 
As for the other acknowledgements of audience, again, some similarity in Composition 2 classes, particularly in the second semester, suggests that audience did come to play a more central role in the decisions the student participants made in their writing toward the end of their compulsory writing education. In contrast, the Composition 1 classes were quite different, with just one mention of a focus on the reader in one class (C), and much more consideration of the reader (with five observed counts) in the other class (F).

The extreme cases of Aya and Satoko regarding the identification of audience best illustrates the effects this particular feature of academic writing instruction can have on students in one class. Although Mr. Doi gave a great amount of time and effort to stressing the communicative aspect of writing through peer reading activities and an extensive discussion of reader versus writer responsibilities, the two student participants in the class had very different responses. Aya's awareness of her audience led to frustration and resistance, while Satoko's led to encouragement and acceptance.

\subsection{How writers guide readers}

Another common theme of audience consideration that emerged from the data was the way in which teachers introduced various signals that can be used to interact with or guide the reader. These signals included topic sentences and thesis statements (as guiding signals), transition signals, 'hooks', and questions to the reader. The teachers tended to put the most emphasis on topic sentences or thesis statements as most important for guiding readers. Thus, the notion of how writers guide readers can be considered a second sub-indicator of audience consideration. Data fitting into this category, therefore, are outlined according to the various signals observed.

\subsubsection{Topic sentences and thesis statements}

Similar to the previous results on peer reading, observations of teachers introducing the notion of guiding readers in the six classes differed greatly. While Ms. Aiba had just two observed mentions of topic sentences and thesis statements in the A and B classes, in the D and E classes there were many counts of Mr. Doi and Ms. Ellis encouraging the students to guide the reader using these features of academic writing. In the Composition 1 classes, while Mr. Clark had just two discussions on clarifying topic sentences, Ms. Ellis had a much 
higher number, spending a great deal of class time on this point. The following diagram shows the number of observed discussions on topic sentences or thesis statements, differentiated by function including making an assertion, clarifying the main idea, providing the content and organization of the text, and connecting to conclusion.

Figure 10: Functions and counts of topic sentence or thesis statement discussions

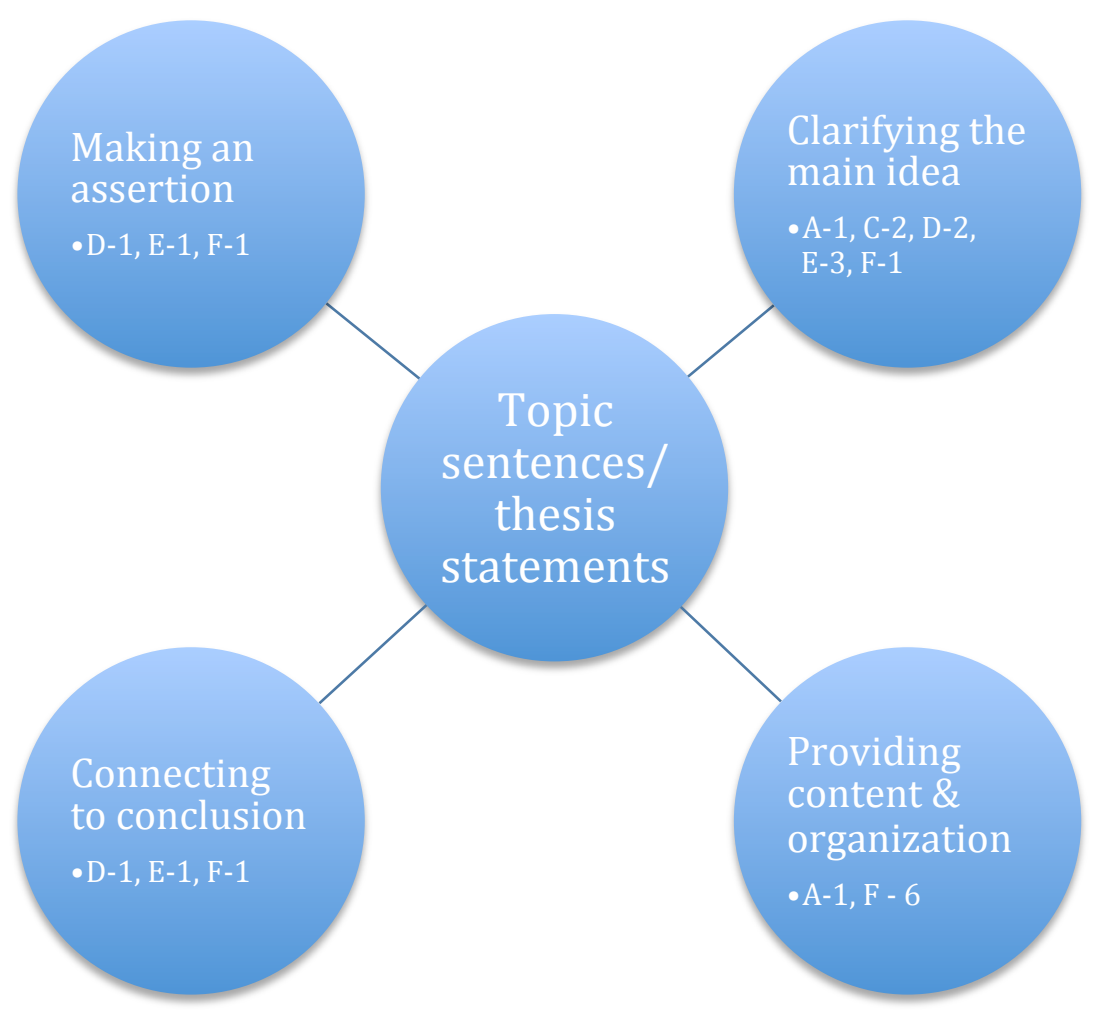

This diagram reveals Ms. Ellis's emphasis on this aspect of academic writing, with the highest number of observed discussions on topic sentences or thesis statements. It also shows the connection similar to the one shown in chapter 5 , where the strongest similarities are shown between Ms. Ellis's (E and F) and Mr. Doi's (D) classes, this time in terms of variety and higher number of discussions on topic sentences or thesis statements.

Early in the first semester, Mr. Doi focused on the importance of making a clear, strong assertion in the topic sentence in order to guide the reader, as illustrated: 
So please keep it in mind that you need not only the topic but a strong, good, detailed assertion in your topic sentence, because, unless you have a good assertion, you won't have a good rest of the paragraph. You need to explain, you need to develop, you need to elaborate what the topic sentence says for the rest of the paragraph, which is today's topic. So, once again, I will repeat... Good topic sentence has one basic idea, one good topic, plus a good assertion. (Mr. Doi, D Class, May 9)

Later in the same class, Mr. Doi connected the topic sentence to appropriate ways to guide the reader and described the functions of its role in relation to a concluding sentence. Ms. Ellis similarly stressed the relationship between the thesis and the conclusion around the same time of year in the E class on May 23, and discussed the importance of the "final statement" of the essay as an important signal for the reader. After that class, Ai explained in her interview that she understood the importance of the conclusion, but the final statement in which she attempted to provide a "future prospect" was confusing for her. She commented:

I'm not good at writing conclusion - always. I can restatement of uh, thesis statement, and then summarize what I, you know - what I... I have argued in my essay, but the last part, um, like future prospect, I always - I had unrelated topics not unrelated but, you know, another topic. (Ai, May 30)

In the $\mathrm{A}$ and $\mathrm{B}$ classes, there was one observed instance when the teacher pulled together a discussion on essay structure (for an application to the Harvard MBA program) by explaining the purpose of the writing task in relation to structure. The teacher emphasized the importance of establishing the organization and content of the essay in the thesis statement:

Ms. Aiba: Remember, Harvard is asking you a question. It's in two parts. It's asking you to identify three events, and they're asking you why those three events are important. So your thesis statement-thesis sentence should include your direct answers to those questions. OK? They ask a question, you answer a question.

Student: $\quad$ So we should have three accomplishments in the thesis statement?

Ms. Aiba: $\quad$ Yes. (A Class, October 10)

The idea of presenting so much information in the thesis statement appeared to present some confusion for Megumi and at least one other student: 
Ms. Aiba: $\quad$ OK, but you have to identify the specific events.

Megumi: In the introduction?

Ms. Aiba: In your thesis sentence...

Megumi: Eh?

Megumi $\rightarrow$ Student: Do I have to do it all in one sentence?

Ms. Aiba: So they're asking you for three experiences, three events. So you say, "My three events are blah, blah, blah..."

Student: $\quad$ So do I need specific events, or...

Ms. Aiba: It should be specific events, and... yeah...

Student: $\quad$ So I need to ... blah, blah, blah

Ms. Aiba: Yeah, I think those are important qualities which you want to identify as specific events, right? In the future...

Student: It's going to be so long... (A Class, October 10)

In the E class there was just one observation of Ms. Ellis explaining how a student's thesis statement written on the board functioned as a signal for the reader. The observed class was on May 23, and the teacher stressed making "specific, concrete, and direct thesis statements." Ms. Ellis suggested the use of evaluative adverbs to help assist in clearer writing and to help students assert their positions in their concluding sentences. This aspect was not the only new idea the students learned about the thesis that they first learned about the previous year in Composition 1. At least one student, Yuki, felt confused about the different explanation of the thesis in Composition 2. "Last year I thought thesis statement should be not so specific. But this year I wonder maybe thesis statement should be very specific. I'm a bit confused" (Yuki, June 1). Thus, emphasis on thesis statements and topic sentences among the four composition classes varied greatly from only a few mentions in the $\mathrm{E}$ class and a brief discussion in the $\mathrm{A}$ and $\mathrm{B}$ classes, to detailed descriptions and explanation in the D class.

As for English Composition 1 there was also particular emphasis given to signaling in certain classes, but not in others. In the $\mathrm{C}$ class and $\mathrm{F}$ class, the teachers emphasized the importance of a clear topic sentence for the reader, as illustrated below in these example excerpts: 
If you have a paragraph, the first sentence has got to be your topic sentence. Otherwise you're going to confuse the reader. At this point, don't make it the second sentence, don't make it the third sentence... give me the topic, and give me the controlling idea... then boom! Roll in the support... OK? (Mr. Clark, C class, July 4)

[Reading from board] "I hate to weed my garden on a hot summer day." Ok, so we know right away, by looking at the topic sentence, that the household chore that [student] hates the most is working in her garden when it's hot outside. Ok? So, it's very clear. So now, we can expect to continue reading and find reasons why [student] does not like working in the garden on a hot day. (Ms. Ellis, F class, June 6)

In other observations, Ms. Ellis continued to highlight the importance of topic sentences and thesis statements in the F class, making a clear connection of the thesis statement to the conclusion, and explaining the functions of various types of thesis statements for guiding the reader through different essay types. In terms of the number of observed discussions of thesis statements and topic sentences, it seems there was much more emphasis in Composition 1 than Composition 2. This became more apparent considering that as the one teacher who taught both levels, Ms. Ellis was observed emphasizing the importance of guiding the reader with thesis statements and topic sentences but was not observed discussing the content and organization aspects of writing thesis statements in her Composition 2 class. She did however make it a clear focus of many of the observed lessons in Composition 1. These results suggest that use of thesis statements and topic sentences as ways to guide readers may be considered by teachers to be a basic skill acquired by the second language writer early in their study of academic writing. This was emphasized by Ms. Aiba's response to overhearing a student ask a classmate about thesis statements:

Student: [addressing a classmate] Nani o [What is a] thesis statement...?

Ms. Aiba: Nani yutte no?! [What did you say?!][whole class bursts out laughing] Did I hear that right? [addressing the class] What's the topic or thesis sentence? Could someone explain to [student] what a topic or thesis sentence is?

Student: $\quad$ Eh! It's the main idea of the whole essay... [class laughs]

Ms. Aiba: Summarized in one whole sentence.

Student: $\quad$ I see... [class laughs] (October 10) 
Clearly Ms. Aiba assumed every student in her Composition 2 class already knew what a thesis statement was.

\subsubsection{Transition signals}

For the Composition 2 classes, it was mostly in the D class where Mr. Doi was observed giving instructions in the use of transition signals to keep readers on track. Early in the first semester, transition signals were suggested as a way to smooth out the argument, and to direct the reader through the writer's ideas. Later in the semester, transitions were reviewed in further detail in this class. In the E class, there were similar observed instructions of transition signals, including one instance in the October 17 class of Ms. Ellis discussing the use of subheadings as useful transition signals for the reader.

In Composition 1, in a second semester observation in C class Mr. Clark made one passing comment on transition signals. However, there was much more discussion in the $\mathrm{C}$ class on the importance of particular structures in essays. Although it was not explicitly mentioned that this was something necessary in signaling to the reader, Mr. Clark was quite explicit with the instruction.

In the $\mathrm{F}$ class, in contrast, there was much more focus on transition signals. In the first semester, on June 6, the teacher was observed stressing the importance of transition words in topic sentences, and offering some lengthy explanations of transition signals. Later in the same class the transition signal instruction moved to concluding sentences, and the teacher also made multiple references to transition signals in relation to connecting paragraphs, making refutations, and emphasizing support. In an interview, Ms. Ellis explained:

Transition signals seem to be one of those things that should be so easy for the students to use, and as much as we practice it in my Comp 1 class, they still don't use them in their essays. So that's why I like to point out that transition signals are used for many different purposes and to help the reader to see all the features and developments of the essay easily. (Ms. Ellis, June 8)

In summary, as with thesis statements and topic sentences, observation data revealed great variation in what the students were being taught regarding transition signals to guide the reader. Moreover, it seemed there was also a division between Composition 1 and 2 classes, where Ms. Ellis extensively taught transition signals to her Composition 1 class, but 
made no mention of them in Composition 2, indicating that learning how to use transition signals effectively, like the thesis statement and topic sentences, may be a skill that teachers expect students to acquire early in their academic writing studies.

\subsection{3 'Hooks'}

A hook is regarded as an essay opener intended to grab the attention of the audience and stimulate their interest in the essay topic, thus encouraging them to read on. Observed mentions of hooks in writing occurred in the F class, where their importance was emphasized greatly by Ms. Ellis. In addition to many other signals, the teacher introduced the concept of the hook at the opening of the essay for the sole function of getting the reader's attention. Then in a later class, the teacher continued discussing the idea of a hook, reviewing with students the various kinds of hooks-questions, observations, quotations, scenarios and statistics. Although not observed in the E class, the teacher did mention hooks briefly as a review of Composition 1 at the beginning of the year. In her $2^{\text {nd }}$ interview, Ai mentioned the importance of hooks to "attract the reader's attention" (May 31), and explained that this was consistent with what she learned in Composition 1.

Though not observed in the C class, Yui also mentioned the importance of hooks in her June interview (Yui, C class, June 21). Mr. Clark had instructed this in an unobserved class.

In the Composition 2 classes, the concept of the hook was covered only by Ms. Ellis as a review of Composition 1, indicating that hooks, like transition signals, were also expected to be covered in first-year academic writing courses.

\subsubsection{Questions to the reader}

The idea of a writer posing a question to the reader directly in an essay clearly indicates the presence of the reader in the writing process. However, it was noted by several teachers (Ms. Ellis, Mr. Doi, Ms. Aiba) that questions were not preferred or allowed, although in every case, no explanation was given. The discouragement of questions was often presented as a rule, without rationale. Interestingly, Ms. Ellis encouraged students to use a question hook, yet discouraged them from using questions at any other time in their essays. However, no explanation was offered as to why a question was acceptable in one position in an essay but not another. Student participants made no mention of this rule in the 
interview data, perhaps an indication of their acceptance of this rule prescribed by their teacher.

\subsubsection{Summary}

Evidence of signaling to the reader was found in various classes in the form of instruction on topic sentences, thesis statements, transition signals, hooks and questions to the reader. However, students were unclear at the time about how to appropriately use these features of academic writing. While the number of observations of this feature of academic writing was low in some classes (A, B and C) but not others (D, E and F), the student participants occasionally discussed this feature without being prompted in interviews, suggesting that these students were concerned about their understanding of what they considered an important feature of their writing.

\subsection{Persuading the reader}

The final notion of considering the audience that emerged from the data, was an emphasis on persuading the reader through use of active or passive voice, either with or without personal pronouns, formal, academic language, explicit description and language clarity, and using sensitivity with language choices, i.e. avoiding potentially offensive language that may be sexist, racist, ageist and so on. The focus on a writer's ability to persuade a reader seemed to lie in writing objectively but actively, with rich, careful description.

\subsubsection{Active/passive voice}

The teachers' instruction on the use of active or passive voice in persuasive writing was inconsistent between teachers. Ms. Aiba explained that a way for students to avoid personal pronouns in making their writing more objective and persuasive was by using passive voice:

In academic writing it's better to avoid using I, and in order to avoid that you can turn the sentence into passive voice. That way you don't have to bother with I. (Ms. Aiba, B class, July 4)

Mr. Clark made four mentions of active voice in the observation data, including a discussion with students over the use of passive voice to avoid personal pronouns, such as we. In this discussion, however, the teacher discouraged use of passive voice in favor of active voice 
which replaces personal pronouns in favor of words such as "the research" and "this paper". The following conversation between Mr. Clark and a student illustrates this point:

Mr. Clark: Passive sentences should be avoided, why?

Student: Because it's boring?

Mr. Clark: It's boring, yeah! The things you're talking about aren't doing anything. We want to see people doing things, animals doing things, things doing things, not having things done to them. It's boring. If it's boring it's going to make your reader go, "Pfft, I don't want read this, nothing's happening!" If things are happening to other things, OK? (C class, July 4)

Thus, we see a discrepancy in the observation data where Ms. Aiba was using passive voice as a writing strategy to avoid personal pronouns, while Mr. Clark was discouraging students from using passive voice in all other cases.

\subsubsection{Impersonal/objective}

In addition to the discussions above where a focus on active voice led to the teacher suggesting that personal pronouns should be avoided, there were a number of further mentions of the notion of personal pronouns in observation data. It seemed most teachers viewed the avoidance of personal language as a strategy to make writing more persuasive and objective. Ms. Ellis specifically stressed this issue, as exemplified in the statements below.

Remember, I don't like personal pronouns. I don't want you to use "I", "we", "us". Ok? I want you to speak in a collective voice as though you were representing everybody else's voice. (Ms. Ellis, E class, 23 May)

Ok, another thing. I ask you to challenge yourselves not to use pronouns like "you" or "us" or "we". Because this particular task asked you what household chore you hate the most, it's ok to use "I" in this particular case, but I want you to avoid speaking in a collective voice, as if you're speaking for everybody, or using "you", because once we start to write more academic papers, you're not going to be able to use those at all, and you're not going to be able to use "I"- only very sparingly. (Ms. Ellis, F class, June 6)

The essay will eventually be written from an objective viewpoint, not the subjective, so you're going to have to take the "I" out of the essay. (Ms. Ellis, F class, June 27) 
A potentially contentious point, the teaching of personal pronoun usage by three teachers was discouraged, in total contrast to Mr. Clark who taught that the use of personal pronouns was persuasive. In the second semester in the C class, Mr. Clark encouraged the use of personal pronouns such as you and $I$. In the observation, the teacher did not suggest a particular reader to whom the essay should be addressed; therefore he did not give a reader to the recommended use of you in the students' writing. This completely contradicted all the other observed instances in the other classes.

My advice, just to keep it simple, OK? You can restate your-“As you can see, we do not have to throw away our video games." Go ahead, restate the main idea sentence. If you have one of these that you think is maybe the best possible solution... "I personally think that exercising an equal amount of time is the best because people will want to jog around their neighborhood if they play video games." If you really think that, you give your opinion. (Mr. Clark, C Class, October 17).

This particular discrepancy between the $\mathrm{C}$ and $\mathrm{F}$ English Composition 1 classes raises a crucial point, as students were being taught opposite information regarding the use of personal pronouns. This would also affect the consideration of the level of formality required for academic writing, which is discussed next.

\subsubsection{Formal language}

There were some observed instances when a teacher placed emphasis on the formality of language use. One of these observed instances was in the A and B classes in the second semester when the teacher required the students to consider the necessary formality of a written text for a particular audience, in this case the Harvard University MBA application review board. Here, the teacher discussed how to "grab the reader's attention" and suggested a formal register was more persuasive:

Ms. Aiba: $\quad$ OK, so don't write something very vague, uninvolving, un-engaging... that's not the kind of writing you want. You want to be specific so you can grab the reader's attention, and vicariously experience what you have experienced. Now, what about the style of writing... Is it informal or formal?

Student: Formal?

Ms. Aiba: Formal? Yes? Yes, for this, for our purpose, it should be formal. Remember we're sending not to a friend, but we're sending it to 
Harvard. We like to be formal. But as for autobiography per se, is it always formal? It depends. Yeah, it could be formal autobiography. Good, it could be very narrative, very casual writing ... (A class, 10 October)

Another point raised by Ms. Aiba in the same class was that although formal language needed to be used, students still needed to provide their own personal style. It was recommended that this could be achieved through sentence structure variation:

Ms. Aiba: [reads] "Would dialogue work in your essay? Would the reader understand the why or the how of the event? Does the sketch conclude or merely quit? Have I used the personal style that offers additional style for the reader? Have I varied my sentences both by length and by structure?"

What do we mean by that? Length - some sentences are long some are short. Which is the more effective one? Longer or shorter? If you want to emphasize something, which is better?

Several students: Shorter.

Ms. Aiba: Shorter. Shorter adds emphasis. (A class, October 10)

Thus, in this class, formal language both in terms of register and sentence complexity was a way in which the audience was considered in this writing class.

In the Composition 1 classes, the observed instances of formal language were not explicitly taught as persuasion techniques, but rather Mr. Clark maintained a focus on specific grammar points (dependent and independent clauses) in order to make the point that the students' writing should be more complex, and thus more academic:

OK, there's a reason I've given you those [grammar] sheets. Why? Because when we get to this part we have to know what a dependent clause is. We have to know what an independent clause is. Those things don't stand together. Plus, we are in the second semester, right? The first semester was the baby semester. I was trying to get you away from these simple sentences. "I like ice cream." "I like pie." "Video games are bad." "Living with parents is good." [class laughs] We're getting beyond that. (Mr. Clark, C class, October 17)

In the $\mathrm{F}$ class, Ms. Ellis regularly highlighted the formality of academic writing. In this example from early in the year formal language was to be achieved by forbidding the use of the word get: 
Do not use "get" or "got", ok? A lot of you guys used "get" or "got" and I specifically wrote on your little papers, do not use "get" or "got", it's not formal writing style, and I want you to challenge yourself to come up with more formal verbs to express your ideas. "Get" and "got" is.... You know, you use it when you're speaking, but you're not using it in writing. (Ms. Ellis, F class, June 6)

In another observed instance the teacher worked with the class to edit vocabulary to make a students' writing academic:

Ms. Ellis: [reads] "It is more effective to get education in school, which has trained teachers, owns useful equipments and offers a suitable environment for studying, than to get education at home." Ok, pretty good. What's wrong? What's a word that I said, "Do not use in this classroom"?

Student: Get.

Ms. Ellis: Yep. So, what word can we use instead of get?

Student: Receive.

Ms. Ellis: Good! (E class, May 23)

Toward the end of the year the last observed instance of this sub-indicator in the F class came up in a reference to the verbs used in introducing evidence from sources:

Student: [reads aloud] "Use precise, accurate verbs to show the author's relationship to his or her ideas."

Ms. Ellis: I can't tell you how often I see in papers, "Dadadada says," "he says", just, you know, ugh, I hate that... Um, nobody's talking about "he's saying" anything. Ok? So, you should become familiar with, uh, verbs like "to assert", "to believe", "to maintain". (F class, December 5)

Thus, the sub-indicator of formal language was dealt with differently according to each teacher. Ms. Aiba focused on language register, Mr. Clark emphasized use of complex grammar structures, and Ms. Ellis encouraged use of academic vocabulary. While each observed instance dealt with the issue of formal language in a different way, the overall goal of these lessons was to assist students in writing more academic and formal essays as a way to persuade the reader. 


\subsubsection{Being explicit (providing detail and language clarity)}

Persuading a reader may be easier if the reader is able to see the ideas raised by the writer. Although detailed descriptions are often reserved for creative writing classes, there is certainly room for providing explicit detail in persuasive academic writing.

For Composition 2 classes, there was one observed count in each of the A and B classes where there was emphasis on providing the reader with rich description and detail. It was the task of writing a personal essay as part of the application to the MBA program at Harvard University.

Page $185 \ldots$ It says, [reads] "Include details that allow readers to feel what you have felt, see what you have seen, think what you have thought." In other words use good description to involve readers in your own experiences... You should almost be able to touch the experience from your description. (Ms. Aiba, A class, October 10)

For the E class, brainstorming activities were the basis of the first observed class of the year, and these were the times when students were the most active in the class. In this class there were many observed instances of Ms. Ellis focusing on the ideas students presented in their writing as she often asked students to put their writings on the board so the whole class could work together on them. In this excerpt from the E class on May 2, it was clearly observed that the teacher emphasized for the students the value and importance of the clarity of language used to express their ideas in the writing process:

Ms. Ellis: $\quad$ Ok, so you know, your idea is good, and I understand what you want to say, but from the example you gave us, we have no idea. Ok? So, think what you want to say, summarize it in your mind, then write it, ok? What's the question? What's going on?

Student: I think it wants to say, not studying outside the classroom, but exercising will help children.

Ms. Ellis: Ok. That's what you think she's trying to say or that what you think it could also say? I don't think that's what she's trying to say, but I think that's another example.

Student: So, I think studying should be emphasized?

Ms. Ellis: You think this should be emphasized? Ok. [writing on board] Yeah?

Student: Uh, I think we can cut "by studying outside". 
Ms. Ellis: Where do you want me to cut? You want me to start cutting here? [laughter] From "but"? You want to cut this whole thing?

Student: Yeah.

Ms. Ellis: "Moving student's own bodies not only keeps them physically healthy, but also is good for mental health." Yep, you can do that too. That makes it more simplified, easier to understand, more straight to the point. Ok, good. (E class, May 2)

For the Composition 1 classes, only Ms. Ellis (F class) raised issues related to persuading the reader. In the first class observed in the year there was the following discussion in which the teacher emphasized the importance of providing explicit and complete information as a way of avoiding plagiarism:

Ms. Ellis: Do you need to let your reader know that you found this information in The Japan Times, or can you just write the statistic without letting the reader know where you found it?

Student: You have to tell them.

Ms. Ellis: You have to tell them. Great, you have to tell them that you got it from The Japan Times, because if you don't tell them, then what are you doing?

Student: Plagiarizing.

Ms. Ellis: You're plagiarizing, all right, very good...Ok, so it's ok to use the work of other people, it's ok to use the research of other people, but when you use it, you always need to let your reader know where you found that information from. (F class, April 25)

Thus, the inclusion of in-text citations was a further way that students were encouraged to be explicit for the reader and to persuade them of their thesis. Being explicit appeared to be a significant feature of academic writing in Ms. Ellis's classes. However, except for Ms. Aiba's mention of it in her A and B classes, this feature was not observed in Mr. Doi's or Mr. Clark's classes, indicating further inconsistency between courses.

\subsubsection{Being sensitive to all audiences}

In two of the observed English Composition 2 classes (B and D), the students had discussions on being sensitive to all audiences when making language choices in writing. In the B class at the end of the first semester, a discussion was observed on shaping writing 
according to social purpose, in the form of "writing with sensitivity". This issue was raised in the set grammar-focused textbook the class was using. The specific writing structure discussed was the use of the pronoun 'he'. This then led into a discussion on avoiding language that marginalizes groups of people including women, disabled, blacks, gays, and children. The teacher used the term "politically correct" and discussed "strategies" for maintaining sensitivity:

Ok, so when we say sensitivity we want to be politically correct, is what you told me earlier, so how do we ensure that our writing is politically correct? What's one way of ensuring that? What are the strategies we can use to make sure our writing is politically correct? ...We just mentioned one earlier, we should avoid the male pronouns because we want to make sure that we're inclusive and not sexist. (Ms. Aiba, B class, 4 July)

In the D class there was a similar introduction to the issue of writing with sensitivity. In this instance, the teacher used the example of using the word "discovered" with Columbus's landing in the Americas:

Student: I think America existed before Columbus sailed over to discover it.

Mr. Doi: Yes. The continent of America existed before Columbus discovered it, therefore, "he discovered it" is very insensitive. America existed before Columbus discovered it. (D class, May 30)

That this feature of writing was found only in Ms. Aiba's and Mr. Doi's classes suggests a potentially significant discrepancy between teachers' approaches regarding cultural expectations, as the two native English teachers in the study were not observed discussing writing with sensitivity.

\subsubsection{Summary}

The fewest observed instances of any indicator were those with a focus on persuading the reader. The examples found here reveal that this may be a crucial area where a writer considers his or her identity in relation to a reader when writing argumentatively. Most significant were the examples of contradictory instruction between teachers on using active voice and personal pronouns, and the inconsistency between courses on teaching students to write explicitly or sensitively. Students who did not receive instruction on writing to persuade the reader may have been disadvantaged. 


\subsection{Chapter summary}

This chapter has served to highlight some of the significant discrepancies between classes concerning the philosophies and goals of the teachers, as well as the content relating to a focus on developing the students' awareness of audience. The discrepancies were found in varying degrees in all three sub-indicators: identification of audience, how writers guide readers, and persuading readers.

In the data identified as the sub-indicator of "persuading readers", the greatest discrepancies emerged, as some teachers' instructions were opposite to those of others teachers. This was particularly exemplified by Mr. Clark's instruction stressing the use of active voice and informal/personal language, in stark contrast to other teachers' instructions on the use of passive voice and formal/impersonal language. However, this sub-indicator showed one important consistency regarding the sensitivity of language between the A/B classes and D class.

Next, under "how writers guide readers", the extreme differences in the amount of stress and emphasis that was put on the various features of this sub-indicator were obvious. In their interviews, students described some confusion about these differences in emphasis, but did not express particular complaints (raised in the next chapter) about these differences.

Finally, under "identification of audience", most teachers tried to incorporate some peerreading (although the $\mathrm{A} / \mathrm{B}$ classes did not), and all classes at various points throughout the year made multiple acknowledgements of the reader, showing important consistency in that writing was seen as a communicative act by all the teachers in the study.

The next chapter will focus specifically on the indicator of the writer's development of the thesis. Some of the ideas raised in this chapter will be further elaborated, particularly in relation to the teachers' instructions on the use of personal experiences, and on the students' commendations, concerns and complaints about course materials and content. 


\section{Chapter 7. More observations: Developing the thesis}

Having provided the foundation for analysis of the observation data in section 6.1, this chapter presents the second part of the analysis. It specifically focuses on how the teachers directed students on aspects of critical thinking such as framing an argument and establishing a position on the argument. This indicator marks any and all observations of class lessons and activities that focused on students' own ideas, opinions, and/or experiences in the development of their writing. The sub-indicators that emerged from the data are shown in the table below. The further divisions were used as the codes for analysis, and form the organization of this chapter.

Table 8: Sub-indicators and further division of the writer's development of a thesis

\begin{tabular}{|c|c|c|}
\hline \multirow[t]{9}{*}{$\begin{array}{l}\text { Development } \\
\text { of the thesis }\end{array}$} & Brainstorming & $\begin{array}{l}\text { Lessons on brainstorming (how to } \\
\text { brainstorm) } \\
\text { Brainstorming as an activity }\end{array}$ \\
\hline & \multirow[t]{3}{*}{$\begin{array}{l}\text { Establishing a } \\
\text { position/claim }\end{array}$} & Reasons for stance \\
\hline & & Counter-argument and Refutation \\
\hline & & Using feedback to establish position \\
\hline & \multirow[t]{3}{*}{$\begin{array}{l}\text { Personal } \\
\text { experience }\end{array}$} & Personal experience forming a thesis \\
\hline & & Personal experience as supporting evidence \\
\hline & & Discouraging use of personal experience \\
\hline & \multirow{2}{*}{$\begin{array}{l}\text { Students' } \\
\text { acceptance/refusal } \\
\text { of materials }\end{array}$} & Teacher encouraged responses \\
\hline & & $\begin{array}{l}\text { Students openly critiquing materials and } \\
\text { content }\end{array}$ \\
\hline
\end{tabular}




\subsection{Brainstorming}

Any time a class becomes focused on the students' own ideas in the development of the thesis, issues of writer identity become central. Brainstorming, for example, requires student writers to generate their own ideas and/or topics. This step in the writing process was dealt with in all observed classes in a variety of ways, depending on the teacher. The different approaches to brainstorming included lessons on brainstorming as a writing skill, and brainstorming as a classroom activity.

\subsubsection{Lessons on brainstorming}

For most Composition 2 classes, brainstorming or focusing on using the students' own ideas were observed. In the A and B Classes, a task required students to write an essay for an application to the MBA program at Harvard University. The essay question was, "What are your three most substantial accomplishments and why do you view them as such?" The discussion in this class focused on generating ideas and creating an outline in order to start the first draft. During this class, students were encouraged to first ask themselves questions to think about how to organize their thoughts, and then create an outline with a thesis statement and all topic sentences included. Then they began to write a first draft as one continuous flow of writing following the outline. The teacher, Ms. Aiba, emphasized that by creating a detailed outline and carefully constructing a thesis statement, the writing process would become considerably simpler and clearer.

In the D Class, Mr. Doi also had the class discuss their understanding of brainstorming, and linked this to the importance of outlining in a similar way to Ms. Aiba. In the observed class the discussion was a revision of the content related to brainstorming in the previous class:

Mr. Doi: $\quad$ Brainstorming. Yes, we did brainstorming. What was it?...

Student: $\quad$...Write up a lot of ideas-

Mr. Doi: $\quad$ Write up, yes, we write up a lot of ideas about, topic sentence - one topic. And what did we do? We put a lot of words around the core idea, and what then we do? What did we do then?

Student: Uh... related the words. 
Mr. Doi: $\quad$ Related those words together, probably we cluster into three or four groups. (D class, May 9)

In the same class, the students were given the task of brainstorming on reader versus writer responsibility in English and Japanese writing. According to the textbook used in class, in English it is the writer's responsibility to make ideas clear to the reader, while in Japanese it is the reader's responsibility to try to understand the ideas. The first step in the brainstorming process was to agree or disagree with this idea. As the students offered their ideas on the topic, the teacher guided them on how to structure their brainstorming to assist in the organization of ideas and creating a thesis statement. He did this by getting the students to first agree or disagree, and then build an outline.

In the Composition 1 classes, the teachers gave more guidance on the brainstorming. For example, in the first semester in C class, the topic of video games was given to the students who were then required to brainstorm their ideas on the topic in order to write a paragraph on it. Mr. Clark directed them through the brainstorming process so they could create a cluster and a controlling idea.

In the F class, Ms. Ellis generated an extensive discussion of brainstorming toward the end of the first semester. First the teacher confirmed the students' understanding of the goal of brainstorming. Then the class did a brainstorming practice starting with identifying a topic. The teacher emphasized the importance of choosing topics the students were interested in, and explained the value of visualizing the ideas about those topics on paper. Multiple ideas for brainstorming were introduced using materials from a textbook, including the cluster diagram, listing, and straight-line cluster. Finally the teacher emphasized the importance of students brainstorming more than one topic so they could decide about the feasibility of writing on a choice of topics.

Toward the end of the year in the C class, Mr. Clark again stressed the importance of the brainstorming stage as a separate and independent stage from outlining. This was to make sure it was clear to the students that they should complete the brainstorming stage before moving on to the outline, and that every idea finalized in the brainstorming stage was to be 
used in the outline. Although Aki completed the brainstorming stage for each essay, she expressed in her follow-up interview that she felt the strictness of the writing task made the brainstorming harder.

Aki: The way to do it is very strict.

Jim: So [teacher] had very specific ideas about what you could write and what you couldn't write. Did you feel like that was helpful?

Aki: No, it made it harder. Especially when doing brainstorming.

Jim: Ok, tell me about that.

Aki: It seemed like separate task from the essay. I didn't really see a relation. I just did it [brainstorming] as [teacher] wanted us to do it, and then didn't use at all ...when I wrote the essay. (June 4, 2008)

Aki then explained that in her Composition 2 class (of which she had completed the first half of one semester), she did not to have to do brainstorming, and felt this made the writing process easier.

To sum up, in terms of brainstorming as a process in writing, all teachers emphasized brainstorming as an important initial step in generating topics, ideas and support for these ideas. Students in these classes were introduced to a number of brainstorming methods, including making lists, organizing ideas into clusters and generating an outline from the results of their brainstorming activities.

\subsubsection{Brainstorming as a classroom activity}

In addition to being part of the writing process, brainstorming was also used as a classroom activity to generate student ideas and opinions in most of the observed classes throughout the year. Many of the observed instances were the teachers' attempts at getting students to focus on their ideas as the most important aspect of their writing.

Mr. Doi initiated a problem-solution brainstorming exercise in the D class in order to extend the ideas students were generating in three separate readings to be used for their essay assignment. For the E class, brainstorming activities emphasized the value and importance of student ideas in the writing process. There were many observed instances such as the following: 
Ms. Ellis: So, what do you think about that? "Kids can learn about various sports which will help them to make foreign friends in the future." Is that better? Yeah? Ok. Any other ideas about how we could use this? Idea of sports as a transferrable life skill? Yep?

Student: Kids learn about sports which give us good manners.

Ms. Ellis: Manners. Ok, good, good. Any other ideas?

Student: Learn about various kinds of sports and enjoy through their lives.

Ms. Ellis: $\quad$ Ok, good. (E class, May 2)

In Composition $1 \mathrm{C}$ class on October 17, Mr. Clark extended a discussion on taking a position as "revealing the problem", and it was therefore the students' task to brainstorm solutions for a problem-solution essay. The students spent most of the class time on brainstorming, much of it independently, in silence. Situations like these, where teachers used brainstorming as a classroom activity to generate opinions and ideas, represented the way in which brainstorming was observed as an important focus in the classes.

\subsubsection{Summary}

Ultimately, brainstorming explanations and exercises were the most consistent feature in the entire study. The students understood that their ideas were central to the writing process and tasks, even when topics were decided for them. Issues that arose in consideration of brainstorming as a pedagogical technique were the impact on students' confidence as writers. Often classes were quiet when doing whole class brainstorming sessions and, except for the D class, few students participated vocally in those sessions. However, the brainstorming exercise in the D class in which most of the students participated required students to brainstorm for the "right answer". This exercise in the D class, along with the lack of participation in the other classes, suggests teachers needed to provide students with workable boundaries to the brainstorming activity to prevent it overwhelming them.

\subsection{Establishing a Position or Claim}

According to the observation data, when developing an argument, student writers will consider the following: support for their stance, how to be analytical (as opposed to descriptive), how to integrate alternative views such as counter-arguments and refutations, 
and to discuss all feedback in order to understand and use it. The following section, examines occurrences of these factors in the observation data.

\subsubsection{Stance}

In the Composition 2 classes where it was expected that students would be developing arguments for the purpose of writing a research paper, there were many observed discussions on establishing a position or claim in the $\mathrm{D}$ and $\mathrm{E}$ classes. However, in the A and B classes there were no observed cases. This is most likely because the tasks assigned in that class did not include an argumentative essay. In the D and E classes, establishing a critical stance was essentially the goal for the students.

Mr. Doi discussed the notion of stance in academic writing very early in the year, prompted by a conversation with a student who was confused about a topic sentence containing assertion:

Student: Assertion?

Mr. Doi: $\quad$ The text said topic sentence equals something with assertion.......Main idea or topic. Topic with assertion. Topic plus assertion is the topic sentence. So if you put just topic, it's not good enough... ..So assertion is the key to a good topic sentence. (D class, May 9)

The discussion then focused on the importance of the assertion, as that which makes a topic sentence good, and the teacher suggested a topic sentence needed to be more subjective and less like a statement giving facts.

Also in the D class, a particular discussion took up a large amount of class time in the observation made on May 9. The discussion was on reader and writer roles and it originated from a sample topic provided in the textbook on writer versus reader responsibility. To initiate discussion, Mr. Doi pointed out the necessity of taking a position of agreement or disagreement, and being able to explain or justify that position. The teacher encouraged the students to consider the supporting reasons for both positions by developing a table with two columns where the students could list the reasons, presumably to balance them. It was again emphasized that students were required to either agree or disagree. This presented a problem for Aya, who explained in an interview: 
My lifetime position I think - I'm always hanging in the middle... I can see good point and bad point always and I think the important thing is make the most of it whether it's bad or good - it doesn't really matter to me. So I was neutral and it seems like people think I don't really have my opinion... I don't want to take any side. (May 30)

As the discussion on the topic became heated it resulted in wide disagreement between students (something the teacher had indicated was encouraged in the class as it showed critical thinking and awareness). The teacher felt it necessary to try to calm the class by summarizing the argument and giving his own position on the topic in relation to his identity. The fact that the teacher, similar to many of the students in the class, did not identify as strictly Japanese but rather a multicultural person and therefore a multicultural thinker, seemed to help bring the argument to a place of some compromise by introducing issues of stereotyping. Though this was perhaps a distraction, for the sake of class control, it seemed to be a successful move on the teacher's part:

Another consideration is that this kind of question is just a propulsion of cultural stereotype. I was born in Japan, grew up in Germany, and I had difficulty adjusting to Japanese elementary school, so I tried hard to be Japanese, but I couldn't. So nationality-wise I'm Japanese but I may speak a little differently, or act a little differently, and I may have a different logic from ordinary Japanese, but I'm still Japanese. Do I fit into this stereotype? ...Another cultural stereotype is this. [Draws a spiral shape on the board] This is the idea... that the Japanese go around, never getting to the point. But English speakers get to the point. Maybe so... When I speak in Japanese, I may go like this. When I speak in English, maybe I go like this. (Mr. Doi, D class, May 9)

In this class, then, the teacher and students brainstormed, discussed and debated the issue of reader and writer responsibility, which led to numerous mentions of the identity of the reader and writer when writing in English or Japanese. Thus not only was the objective of this class relevant to the study as the teacher was encouraging students to develop a stance in their discussions, but the content of the actual discussions were also revealing because they showed that issues of identity in writing were very important to both the students and the teacher.

Ms. Ellis also conducted classes in a similar fashion to Mr. Doi; activities in her E class were also used to generate opinions on a topic. Because Ms. Ellis often had students write their ideas for thesis statements on the board so the whole class could discuss them, there were 
several observations of developing stance in student writing. The first cases were from a class early in the first semester in which the students were given the topic of animal testing and were required to come up with example thesis statements both for and against positions. This activity, therefore, was similar to the activity in the D class, where students considered both sides of a topic before establishing a position. This raised some concern for Yuki, who was not confident about this "specific" type of thesis statement.

Yuki: ... only problem is thesis statement.

Jim: Ok. What about thesis statements?

Yuki: How specific it should be.

Jim: Oh, ok.

Yuki: There were a lot of explanations on it, but I still feel unsure. (June 1)

Like Mr. Doi, Ms. Ellis also encouraged students to look at thesis statements from both sides of an argument in order to discuss and develop an opinion. In a class early in the first semester, Ms. Ellis emphasized the importance of providing support for a thesis in order to establish clarity of the position:

Usually for something to be obvious, for something to be clear, you would want to introduce support. The only inkling of support there is that animals are the same as humans, but this is a thesis statement, and so you just want to be very direct and straightforward and say what your position is on this paper. (Ms. Ellis, E class, May 23)

Around the same time, the D Class was also looking at stating a clear thesis, but in the form of the topic sentence. In the following observation, the focus was on structure. The discussion centered on how establishing a position in a clear topic sentence allows for easier development of the paragraph. The topic was on kinds of "bad lies":

We learned structure. And then ... we thought it's necessary to develop... What did we develop? This into this, right. What part of this would develop into this? This is... [writes on board] topic... plus assertion. This we develop into supporting data. How can we develop? ... The more important thing is if you could develop the topic sentence which says, "There is more than one kind of bad lie", and giving the reader three different kinds of bad lies... it's good. (Mr. Doi, D class, May 30) 
The teacher then instructed the students to think about the purpose of the activity, with an emphasis on the students' ideas being the exigency for writing, rather than just another task requirement.

During a much later observation in the E class, Ms. Ellis explained that students needed to decide on their own topics. She also explained that the topics should be controversial, and that students should be able to clearly present both sides of the argument:

This semester you can ... choose anything ... you want to write about. So, what I want you to do is start brainstorming that. Brainstorm the topic, think of a research question, and make sure that you can answer "yes" and "no". It has to be a controversial topic. It has to be an argumentative essay. So that means it needs to have two sides. (Ms. Ellis, E class, October 17)

As can be seen in this example, Ms. Ellis approached the notion of stance in academic writing in a similar fashion to Mr. Doi when addressing her Composition 2 class. With so much focus on stance and assertion in writing in these classes, the absence of any mention of stance in the A and B classes became more apparent. This highlights yet another inconsistency in the curriculum between the classes that were observed.

In the Composition 1 classes, teachers took a much more fundamental approach to the issue of stance, but in two very different ways. In the C class, Mr. Clark's focus was strictly on the topic sentence, referred to as the "main idea sentence". In this class there was emphasis on focusing the controlling idea in the topic sentence so that the supporting sentences clearly develop that one clear idea in the paragraph. Similar to Mr. Doi's and Ms. Ellis's Composition 2 classes, Mr. Clark encouraged students to choose a side of the argument. One issue with this was that it caused confusion toward the end of the semester when the students were beginning to extend the organization of a paragraph into the organization of an essay. With this particular focus, students were under the impression that an argument essay should not include a counterargument. When the teacher introduced the notion of examining both sides of an argument in an argument essay, students became confused by the shift in focus. Even at the start of the second semester, using the video games topic as an example, Mr. Clark continued to focus only on supporting the thesis. In her follow-up interview, Yui was asked to explain the organization of an 
argument essay she wrote for the class, and it was clear that her essay had only supporting ideas.

Yui: We used the same outline sheet for every essay ... so we always had to have three like reasons and one conclusion paragraph... so this was our way of organizing the content for an argument right... so I'm arguing that there are advantages for university students living with their parents. I gave three supporting ideas for that and then gave it a conclusion.

Jim: All right so but... did you talk about other ways of organizing these essays?

Yui: $\quad$ Not really... It was always the same so it was just stating what you think in the first paragraph and the reasons like always three reasons. (June 4, 2008)

Mr. Clark placed clear emphasis on establishing a stance based on a collection of supporting ideas, but did not discuss the impact of opposing argument. Instead students remained under the impression that providing a counterargument was not necessary. Student participants were surprised to find their argument essays had been marked down for not including opposing arguments. Although they seemed to understand the value of providing them, since it was not practiced in class, they thought they did not need to include them. Thus, the analytical aspect of argumentation was somewhat lost on these students.

\subsubsection{Being analytical (versus being descriptive)}

Argumentation requires critical and analytical skills from student writers beyond the factual (often descriptive) level of writing, but with the varied approaches of the teachers in this study, students learned vastly different ideas of just how much analysis was involved in the process of developing an argumentative paper. In an early D class, Mr. Doi emphasized the point that being analytical was more important than being descriptive, therefore putting even more emphasis on the importance of the students' own stance in their writing:

From the very beginning of this class I said it's better to be analytical, than be descriptive. Please analyze, rather than describe. In order to analyze something you sometimes need to be angry. (Mr. Doi, D class, May 30)

In the same class, the teacher introduced a new sample topic for discussion, one more familiar to the teacher who was an American History scholar. The topic was on Columbus's "discovery of America" and was used as an example of how history needed to be analyzed. 
The students offered their ideas challenging the statement. The discussion came to a point where the teacher was able to point out that the students' challenge of an historical "fact" was an example of analysis and "active thinking".

Finally the teacher offered a personal experience of trying to be analytical as a student. The anecdote raised issues of being "emotionally engaged" in writing in order to be analytical. The point emphasized the importance of students' personal engagement in their writing:

[My professor] said, "Today, I wonder why you graduate students are not angry." $\mathrm{Hm}$ ? Why do we historians have to be angry? It's about this. In order to analyze, or in order to be analytical, sometimes we have to be angry-emotionally engaged. You've got to have some kind of strong reaction to a fact or an interpretation. With that, you can get away with it. If you do not have any emotional reaction, you have no energy to be analytical... (Mr. Doi, D class, May 30)

In the E class on October 17, the notion of being analytical arose during a discussion on the topic of euthanasia. Ms. Ellis tried to frame the argument behind euthanasia in such a way that students could establish a position on it. In the observation, the teacher did a lot of thinking aloud, which demonstrated the importance of analyzing an argument before writing about it. It was clear that the teacher was encouraging students to be analytical in their approach to a topic when establishing their opinion.

\subsubsection{The counterargument and refutation}

Although in the previous section for Composition 1 classes it was noted that in the C class, the teacher mainly had the students focus on choosing one controlling idea, and supporting this idea through factual description, the F class undertook quite different writing tasks. For the F class, students were presented with a much more analytical writing task than other classes. The students' main focus from the beginning of the year was on developing an argument essay, rather than fundamental organization and structure. This was quite different from what happened in the C class.

The teacher emphasized the importance of understanding both sides of the argument, with particular focus on presenting the counterargument in an argument essay. This was explained in the first semester in the observed class on June 6. In the same class the teacher then connected the development of the argument essay to the writing process, with 
emphasis on brainstorming the counterargument for inclusion in the content of the essay. In the next observed $\mathrm{F}$ class on 27 June, the teacher returned to the focus on counterargument in preparation for students' individual consultations on their argument essays. Students were required to explain all the parts of their argument essays, including the refutation to the counterargument that was taught in an unobserved lesson.

In the second semester in F class in the observation on October 10 the focus turned to the task of writing an argument essay for which the students chose the topic. The teacher took a moment to encourage students to choose controversial topics (the class had used examples from a textbook on debates in the first semester) in order to stress the importance of establishing two opposing sides in the argument. Once again, the counter argument was reviewed and emphasized. In that same class the teacher put the focus on the refutation as a function of persuading the reader. The teacher described the task for the students to present an evaluative refutation to help develop the thesis:

Ms. Ellis: And then, in order to persuade your reader that your idea, that your position is stronger and better than all those other people who don't share your idea, what do you need to do with their counterargument, what do we do? To persuade the reader?

Student: By writing the refutation.

Ms. Ellis: Perfect, excellent. By writing the refutation. All right, very good. So, to write your refutation in, you explain that even though other people do have this idea, it's not the best idea. And it's not an idea that should be regarded more highly than the one that you have. And that will make your argument persuasive. If you don't let your readers know that you're thinking about what other people might think, then your paper will just sound like your opinions, like your propaganda. And it won't be taken seriously. (F class, October 10)

In that same class the teacher went on to provide a specific organization for the body of the essay in which all supporting evidence was presented first, followed by the counterargument.

Thus, a large amount of class time in this $\mathrm{F}$ class was devoted to the notion of the counterargument, which was in contrast to the $\mathrm{C}$ class. Mr. Clark stressed that students 
should simply "add" their opinions in the "main idea sentence" and concluding paragraph, and make some kind of final comment in the form of a suggestion or solution.

\subsubsection{Establishing a position through discussion of feedback}

A fourth way that the theme of establishing a position emerged from the data was in the use of feedback as part of the process. That is, while in the previous two sections establishing a position was a classroom activity or step in the writing process in the initial stages of positioning oneself to establish a stance on a topic, using feedback repositioned it as an activity that also occurred later in the writing process. Feedback from peers (discussed in section 6.2.1), teacher feedback, and collaborative feedback involving peers and the teacher occurred to varying degrees in different classes. The following table shows the number of the types of feedback coming from the teacher, peers, or the class as a whole, differentiating between written, spoken or interactive feedback, and other mentions or attention to feedback that occurred in observed classes.

The table below (Table 9) shows that the types of feedback varied depending on the teacher. Ms. Aiba's (A and B) classes involved only one class in which written feedback on a task related to developing a thesis was a collaborative class activity. The situation in Mr. Doi's (D) class was unique in that the students did much of the feedback themselves, most often spoken by a single student in response to a presentation of another students' research paper draft. Mr. Doi also discussed the importance of using feedback in establishing a position, and on two occasions offered spoken feedback on this point to the whole class. Mr. Clark's (C) class spent a portion of time on interactive and written feedback (though fairly general and not specifically only on establishing a thesis) in two separate observed classes. He also discussed feedback for this purpose twice, and provided written feedback once for students in class. Finally, Ms. Ellis's classes (E and F) were fairly

similar in that she provided some discussion of feedback and spoken feedback on a student's thesis in class. Also, the interactive collaborative feedback on students' thesis statements done in class occurred quite often in the E class. This aspect was unique to Ms. Ellis's classes. 
Table 9: Types of observed feedback

\begin{tabular}{|c|c|c|c|c|c|c|}
\hline \multirow{2}{*}{$\begin{array}{l}\text { Туре of } \\
\text { feedback }\end{array}$} & \multirow{2}{*}{$\begin{array}{l}\text { Level } \\
\text { Class }\end{array}$} & \multicolumn{3}{|c|}{ Composition 2} & \multicolumn{2}{|c|}{ Composition 1} \\
\hline & & A/B & D & $\mathrm{E}$ & C & $\mathrm{F}$ \\
\hline \multirow[t]{3}{*}{ Teacher } & Spoken & & 2 & 1 & & 1 \\
\hline & Discussed & & 3 & 2 & 2 & 1 \\
\hline & Written & & & & 1 & \\
\hline \multirow[t]{3}{*}{ Peer } & Spoken & & 10 & & & \\
\hline & Interactive & & & & 2 & \\
\hline & Written & & & & 2 & \\
\hline \multirow{2}{*}{$\begin{array}{c}\text { Collective } \\
\text { (Teacher }+ \\
\text { whole class) }\end{array}$} & Interactive & & & 9 & & 3 \\
\hline & Written & 1 & & & & \\
\hline
\end{tabular}

There was one observed case of Ms. Ellis assisting a student in understanding the feedback offered by a peer on an argumentative essay on the topic of euthanasia. Although a very basic example, it shows the value the teacher placed on peer feedback by the amount of time and energy spent on helping one student during class time.

Ms. Ellis: " "...there is no evidence that people are better off dead." Ok, so her argument here is that she's against euthanasia. Ok, and then you say here, she probably misunderstood the issue about euthanasia. The issue is not about which is better, death or living in distress, instead it is about which is better, dying in agony or dying peacefully and quietly. Since people's sense of value towards life and death is different from each other, depending on religion, culture, the time and the environment in which they are raised. Then what you need to do here is put, "however".

Student: Here? 
Ms. Ellis: Right here, right. "However". Because this is you introducing your counterargument... (E class, October 17)

Through feedback, the student was encouraged to strengthen a previously written stance on the topic by clarifying the counterargument and refutation.

In the final class of the year in E class the teacher summarized the course in order for the students to think about the whole process involved in writing a research-based argumentative paper. Students were reminded of how documenting sources correctly, clarifying counterarguments and refutations, and peer evaluation all form the greater process and that by the end of the year, their writing should have improved because of their understanding of that greater process. The final observation of this notion in the $\mathrm{E}$ class came in the form of a reminder that the students were required to use the teacher's comments and feedback, and to submit the draft with that feedback with their final revision. The student participants in the class had different feelings about this. Nana felt overwhelmed by the process. In her end-of-year interview, she explained:

[teacher] taught us the basic things about argumentative essays, and she gave us the proper feedback to us. But I think that there's something missing between this process. I think we need some more kind of exercises or activities or... I wanted some kind of process like write one paragraph and submit, get feedback, then after that the second paragraph, kind of like that. So we were given the task and have to write the complete essay. That was hard for me. (January 22)

Conversely, Ai felt the process worked. In her follow-up interview, she commented:

I was able to use the feedback I got from [teacher]. Especially for the... conclusion part, yeah. And I think she correct many part of my conclusion. (June 4, 2008)

As for Composition 1, there was one particular instance in the first semester in which Mr. Clark pointed out first, the importance of using teacher feedback on drafts, and second, the importance of brainstorming. This instance was also observed of Ms. Ellis, but this time in the F class. The emphasis on teacher feedback was only one statement, but a crucial one: "You need to understand why I made those comments or suggestions; you need to address those comments and suggestions, and rewrite your thesis statements" (Ms. Ellis, F class, June 27). Thus, in terms of using teacher or peer feedback as a means to re-evaluate student position on a topic, observations in both cases were found in the classes of Ms. Ellis, 
who placed great emphasis on providing students with feedback on their drafts in her classes.

\subsubsection{Summary}

It greatly depended on the teacher how far these writing classes went into the development of a thesis. For two Composition 2 teachers (Mr. Doi, Ms. Ellis), argumentation was a central focus of the class, so the establishment of a position or claim was the basis for the justification to spend a lot of time and energy in class on explaining argumentation and refutation. For the Composition 1 classes, this was yet another point of departure between them, in which one class (C) took a very fundamental approach to the idea of a thesis (without actually referring to it as a "thesis") and did not work on an argument essay until the end of the year, while the other class (F) moved very quickly into the argument essay task right at the beginning of the first year. This difference between the $\mathrm{C}$ and $\mathrm{F}$ classes is particularly illustrative of the "text-oriented" focus, which sees writing as an outcome, and the "writer-oriented" focus, which sees writing as a process (Hyland, 2008, discussed in section 2.1.6), requiring very different approaches from students in their writing.

\subsection{Personal experience}

The use of personal experience as supporting evidence is a contentious point in the teaching of academic writing since it inevitably makes writing more subjective. More subjective writing was often equated with less persuasive, less academic writing in the observed classes in this study, but not all teachers shared the same philosophy on this. This sub-indicator has three further divisions. The first marks observed instances when the teacher stressed the use of personal experience in order to form a thesis. The second includes observations of discussions focused on the use of personal experience as supporting evidence. The third marks any time students were told that in order to make their writing more objective, they were to avoid using personal experience.

\subsubsection{Using personal experience to form a thesis}

The use of personal experience in forming a thesis was not always discussed, but in two classes there were observed instances of the teacher's concern about the students' lack of personal experience with topics that may have been less familiar for Japanese students. The 
issue of teachers assigning students to write on unfamiliar topics was raised by Stapleton (2001) as a matter negatively affecting students' ability to display critical thinking in their writing, discussed in section 2.2.2.

In Composition 2, one particular assignment in the D class required students to write on a topic from American culture. Class discussion on the task focused on a particular aspect of the writer's voice or identity. Mr. Doi stressed the importance of the students considering their own identities in relation to the assigned topic of Barbie dolls, which may have not been familiar to some students who had not had much exposure to American culture. The teacher explained that although some students may not have ever heard of Barbie dolls before this task, personal experience was not required for this task. Rather, through use of the readings they could still form their own opinions and positions on the topic. In her interview, Aya pointed out, while trying to explain the assertion she wanted to make in her essay, the difficulty she faced without any personal experience with the topic:

I think I'm going to use that disabled Barbie as my evidence of representing the social problems. The problem is I don't know the background. I've never been to America and I don't know anyone from America. (June 28)

Eventually, Aya decided to focus her essay on the negative image the disabled Barbie had in Japan-negotiating a way to make the topic relevant to her own experience of playing with American toys.

In Composition 1, at the start of the second semester in $\mathrm{F}$ class when the students were given the task of writing an argumentative essay on a topic of their choice from a list of topics in the first semester, the teacher stressed that personal experience would be useful in choosing a familiar topic that would be easier to write on:

[Speaking to one student] ... more specific crimes in the home, you know, like robberies, and things like that. Drive-by shooting, where people drive by in their cars ... [addresses class] It might be hard for you to think of some examples, because Japan doesn't have the gun laws that the United States has, and this particular essay is specifically about US history, and guns in the US. But it might be hard for you to come up with examples if you're not familiar with the type of crime that's associated with guns in the US, so try your best. (Ms. Ellis, F class, October 10) 
Stapleton's (2001) argument that a lack of familiarity with assigned writing topics negatively affects students' ability to display critical thinking is particularly significant here. If the topics are not relevant to the students' personal experience, they will not be able to develop their writing skills as much as they could with more familiar topics. According to Stapleton (2001, p.530), "content familiarity powerfully shapes both the range and depth of argumentation, consistent with theories on schemata and knowledge structures."

\subsubsection{Using personal experience as supporting evidence}

The use of personal experience as supporting evidence was observed once in Composition 2, but was discussed much more in both Composition 1 classes. In Composition 2, early in the first semester of the E class on May 2, the teacher instructed the class to practice using evidence to support and develop an argument. The focus early in the year was simply on support, in this case personal evidence, rather than the type of support.

In the Composition 1 classes, again a great discrepancy was noted between the two classes. In the $\mathrm{C}$ class there was one observed instance of using personal experience as supporting evidence. It was early in the second semester in a discussion on the problem-solution essay task. Quite distinctly different from the F class, in the C class the students were told that they did not have to do research for the task, and that the focus of the essay could be on a personal problem. They needed to use their imagination, rather than research, to develop the solutions in the essay:

Mr. Clark: Your problem, you decide the problem. You decide the problem and you decide the solution.

Saki: $\quad$ Eh?

Mr. Clark: OK? The boys in this class... there are five boys. The boys - what are boys' problems? [class laughs]

Student: That's obvious.

Mr. Clark: That's obvious? Being surrounded by girls?

Student: $\quad$ Yeah. [class laughs] 
Mr. Clark: Could be. No, seriously whatever problem that you have. Be reasonable, but it can be a personal problem, it can be a Tokyo problem, it can be a country problem, it can be a world problem. OK? You do not have to do research for this. [Student: Yes!] You do not have to go to the Internet, "Ugh, how do you fix this problem, what could be a solution?" You are free to do so if you want to, but, you should have some imagination. (C class, October 17)

When asked about this in her interview, Yui explained that she liked writing using personal experiences, but not for the types of essays she was assigned.

What he does is just more of what you think based on what you are - who you are. Everything is like ideas that - the topic for the exam is it's hard growing up - adults have more work than kids or something like that. It's all based on what you think so I just listed that adults have more work and no time to play, but I would like to write something that I experienced as a - like a different kind of writing... how I can present my - what I experienced in my life as an interesting essay. (December 11)

In a completely opposite approach, in the F class students were advised from the beginning of the year that the evidence used to support their ideas in their essays should be from outside sources such as The Japan Times, using facts and statistics. Then, later in the first semester the teacher stressed that in addition to outside source information, personal reasons or explanations were also useful in supporting and developing their essays:

Supporting sentences are information which may include facts or reasons or explanations, um, detailed information that support your topic sentence. And for this particular task, what you needed to give me were reasons. Reason 1, reason 2, reason 3. Ok? That's what I was looking for. Some of you did not give me any reasons to explain why you hated doing whatever it is you wrote about. Some of you told me what you hated doing, but then you went on and talked about something completely different. (Ms. Ellis, F class, June 6)

Later in that same class Ms. Ellis explained that facts from outside source information would be stronger than personal support:

What I'm looking for is facts, specifically facts that back up the main idea, or reasons, explanations, detailed information, ok? (Ms. Ellis, F class, June 6)

In the F class observed on June 27, Ms. Ellis gave an example of using personal experience as support in a brainstorming exercise. The students were required to come up with their own topics, so the teacher gave the example of tattoos as a potential topic. The ideas used 
in the example brainstorm were all from personal experience. It was not explained to the students whether or not they could actually use those ideas as supporting evidence, or if they needed to do some research to find supporting evidence from outside sources. It concerned Akiko when Ms. Ellis told them at the end of the class that all the rules she had gone over in the semester, including those on evidence, would be in an exam in the next class. When asked about the rules of supporting sentences in her interview, Akiko responded:

Supporting sentences gives readers facts, examples, more specific information... and reasons... I didn't think there were so many rules. I cannot write anything with all these rules. It's too hard... I'm wondering if I can even use them. (June 27)

In that same class (June 27) Ms. Ellis also stressed the use of source evidence from journals, newspapers, books, interviews, television shows, or documentaries. Since it was the first semester, the teacher decided that the students would not have to introduce the sources, but that once they learned how in the second semester they would then have to. The focus at this point was on accessing and using information from outside sources, rather than relying on personal evidence.

\subsubsection{Discouraging use of personal evidence to make writing more objective}

This particular point was observed in the Composition $1 \mathrm{~F}$ class. On two different occasions the teacher clearly stated that the writing task the students were working on at the time was not about personal experience, but that it needed to be objective. The teacher stressed that a thesis would not have been decided until after doing some research and evaluating the source information. Once that was done, the teacher explained, then the students could establish their own position. This point introduced some confusion and concern for the students in the $\mathrm{F}$ class as evaluating information and then forming an opinion was not a familiar approach. Students were instructed that rather than form an opinion before doing any research, the opinion would be formed by what they found in the research:

And then, signing up voters ...register new voters-this isn't about personal experience, ok, it's not about your personal activities, or whatnot. It's objective. You don't have any reason to feel one way or another, until you do research and have evaluated the information available to determine whether or not the evidence leads 
you to be in support of the idea, or leads you to be against the idea. (Ms. Ellis, F class, June 27)

The other observed instances in the F class were toward the end of the second semester on December 5 , in a class on summarizing information found when researching. The emphasis at first was on the fact that a summary should not include personal judgment or any subjective opinion. The teacher then went through the materials provided for that class on summarizing to further highlight the distinction between writing summaries and writing personal evaluations. Hyland (2002b, discussed in section 2.2.2) recommends that rather than oversimplify the issue by teaching students to write impersonally, it would be more effective for students to learn how different disciplines approach academic writing in order to recognize the options for subject-specific writing.

\subsubsection{Summary}

The use of personal evidence in academic writing was perhaps the least consistent of all the sub-indicators between classes with the same course title. For the Composition 2 classes, even in the classes where the students were required to use personal experience, the point was not raised about any general understanding of the relationship between personal experience and academic writing. In the other two Composition 2 classes, there was a great deal of emphasis on using source information, but some discrepancy between teachers on when and how personal experience would play a part in the formation of an argumentative essay, although they seemed to agree that personal evidence was useful in forming the thesis. For the Composition 1 classes where we have so far seen great discrepancies, here it seems they were even greater. Ms. Ellis stressed very strongly from the beginning that students were to be objective in their writing and not use personal experience as supporting evidence, although no explanation of why this was necessary was observed. Later that teacher then introduced the role that personal experience could play in writing for that class-in the stage of choosing a topic. Mr. Clark did not give a description or delineation of personal versus source evidence as support in academic writing. Students in the $\mathrm{C}$ class expressed confusion about it as a result. Ultimately it seems that we can take this as an example of a perhaps overlooked aspect of academic writing in need of clarification and standardization. 


\subsection{Resistance to/acceptance of materials}

As discussed in section 5.4.2, the teachers' decisions in designing their courses help to answer research question 3: What are teachers' identifiable cultural expectations of EFL writing? Here, the students' resistance to or acceptance of the course materials further assists in answering this research question.

In section 2.2.2 on Japanese students' critical thinking abilities, the example of Casanave's (2002) test subject Yuko provided a case where the student's lack of familiarity with her topic of International Relations led to a certain resistance to the provided materials in that she was unable to work with them. The students in this study experienced similar issues, but at both ends of the spectrum. Students who were not familiar with the writing topic, or with the particular skills or features of academic writing their teachers expected of them, were more likely to resist materials on the topic or using those skills or features. An example is Aya's resistance to the textbook that required writing an essay on Barbie, discussed in section 7.3.1. Conversely, students who were already familiar with the topic, or skills or features required, also resisted the materials or writing tasks. An example of this is Yui's frustration in the first semester, feeling the course was going too slowly and she was not developing her writing skills, discussed in section 7.4 .2 below.

Students' assessment of materials can affect the value they give to asserting their opinion in a critical argument. Outside the skills and genre discourses of writing, Ivanič (2004) also describes the social practices discourse of writing in which " $[\mathrm{w}] \mathrm{riting}$ is purpose-driven communication in a social context" (p.234). This discourse refers to "academic socialization", or developing an understanding of disciplinary culture through discussions and focus on students' ideas and-especially in disciplines such as humanities-critical thinking in developing critical argument. This type of classroom is "writer-oriented" (Hyland, 2008). In an attempt to move outside the prescribed skills and genre discourses set by their teachers, students did on occasion challenge the materials in order to establish an understanding of the social purpose of the tasks they were given. This sub-indicator is broken down into two parts: first, the observed instances of the teacher asking for students' opinions of the materials, and second, the students openly giving their opinions. 


\subsubsection{Teacher encouraged responses}

In the A and B classes, students had highly structured lessons directed by textbooks. Early in the year, the teacher did at one point very briefly ask for the students' opinion of the textbook, only to then quickly follow it with some justification:

Ms. Aiba: So this semester I'd like to make sure that you have the grammar, spelling and punctuation all done down pat before we go ahead and do lots of writing assignments. All righty? How are you liking the book so far? Do you like it? You hate it? So-so? I don't care?

Several students: So-so...

Ms. Aiba: $\quad$ So-so. OK [everyone laughs] I'm hearing so-so... OK. We kinda-I don't know if you noticed, the readings this week, she uses a sense of humor so that comes through on and off, so... anyway, yeah, I hope you enjoy it. I chose it so it would be a nice reference book. Once you graduate and have to consult some reference, this would be a decent one. (B class, May 9)

In an attempt to make grammar more palatable for her class, Ms. Aiba assigned students in pairs to give a presentation on the chapter set for each grammar lesson. In her interview, Miki (B Class) was asked about her expectations.

Jim: Is the class meeting your expectations?

Miki: That's a definite no.

Jim: So what kind of stuff have you been working on?

Miki: We read this book Grammatically Correct and then she goes through it, I mean, you've seen it, right?

Jim: Yeah.

Miki: She goes through it and then we have to like, someone does, a presentation based on the text that we've read. And then after that there is an exercise waiting for us. Then that's it. (May 31)

Later in the interview, she explained that the presentations were not effective:

We all do the same stuff, we have to read the same pages, but... what people get out of it is totally different. I hate to say this, but some people can't answer the questions. It's all based on the book... Some people aren't getting it, and it doesn't tell you that you have to get it. The presentation doesn't really help. (Miki, May 31) 
There was a similar case in the D class, but with much more time and energy spent on elucidating students' feelings about the materials. At first the teacher was responding to some resistance to the materials expressed by the students in their reflection sheets on a task prescribed by the textbook.

You're engaged, you're committed, you're into it, and therefore you had a very strong emotional reaction to it... dissatisfying categorization, classification... So for that I appreciate. And also from the very beginning of this class I said... it's better to be analytical, than be descriptive. Please analyze, rather than describe. In order to analyze something you sometimes need to be angry. It may not make sense to you, but think about it. (Mr. Doi, D class, May 30)

The teacher went on to encourage critique of the materials as a way to further illustrate the importance of having a strong opinion.

You told me he [the author of the textbook] has the wrong opinion, the wrong interpretation, he is WRONG! Why, I ask? And you said, it's because of this and that. It's analysis... ...So, please make clear that critical mindedness... Please keep in mind that sometimes being angry is good... against me, against textbook, or against whatever system... it's important. (D class, May 30)

Then the teacher raised the point that being critical of the materials should allow the students to have a better understanding of the intention the teacher had in using them. Aya was intimidated by this activity in class.

Jim: You were talking a lot in class about supporting your critical thought - or the class' critical thought - about the textbook. So can you tell me what that was about?

Aya: That was scary. That was last week. We had a task classifying how to develop the paragraph - and as a classmate said, it's not really good exercise to do because it doesn't have one correct answer for the paragraph. It had two or three or even everything - that the paragraph contains every method - so they thought it wasn't really good exercise and the textbook is not making any point of what it wants us to do... But I think - I didn't say anything because it was scary. (May 30)

Aya's intimidation was indicative of an unwillingness to assert her own opinion, even though she seemed to have one. She commented further in the interview that she did not necessarily feel the textbook was appropriate for the class: 
I think it is important to have a teacher like [Mr. Doi] using textbook and teaching us what the paragraph is all about... Well, I think I don't know - it was too Japanese thinking textbook. Well, he's teaching us lessons in English but the textbook but the author is Japanese... So Japanese language and English language is very different and I... Maybe the author was Japanese-thinking man. (May 30)

It was evident that Aya had a critical opinion of the textbook. Mr. Doi had decided to use the textbook (to which he had no particular attachment) as a way to encourage strong opinion. It seemed the exercise worked to an extent for Aya, but the experience of classmates aggressively voicing critical opinions in class was uncomfortable for her.

In the Composition 1 classes, there was one observed instance in each class of the teacher asking for students' assessment of the course content. In the C class there was one very brief attempt to get student feedback on one particular classroom practice-peer reviewing:

Has peer reviewing been helpful? Yes... I see lots of nodding heads. Peer review has been helpful. (Mr. Clark, C class, December 19)

Then in his interview the next day, on a discussion of the students using feedback on their revisions, Mr. Clark suggested that the positive response he received from the class the day before was not sincere:

I doubt ... that many students will do a peer review. They'll just end up - ok, here's this paper and it'll have this spelling and grammar and stuff. (December 20)

\subsubsection{Students openly critiquing the materials/content}

All of the Composition 2 classes had at least one observed class in which students openly critiqued the course materials or content. In the D class, the discussion grew from the earlier teacher-initiated student critique of the materials, and in particular, one questionable task statement-about reader versus writer responsibility in English and in Japanese. At first, Aya seemed to accept the teacher's justification of the task as it was elaborated:

Aya: $\quad$ I agree with this um, opinion that English writing has-the writer has responsibility to make them understand what they're saying. Uh, I think the structure is different, the shape of paragraph... ...That's-I think that's why it makes readers a bit complicated about 
understanding, because it, um, sometimes writer intentionally disagree with his own idea.

Mr. Doi: $\quad$ Ok, she used the word 'structure'. Structural difference between English language or English expression and Japanese expression. Structure of paragraph maybe? Structure of sentence? Or even a structure of logic. Ok, structures are different. So therefore you agree...

Aya: $\quad$ Yes.

Mr. Doi: $\quad$...that Japanese sentence structure, paragraph structure, Japanese structure of ... logic, do not require to put conclusion or the main idea at the very beginning. So you're suggesting, the logic, some are suggesting - the way the structure-

Aya: $\quad$ Most of the books are like that.

Mr. Doi: $\quad$ Yeah, structure. Interesting. Um, we can go on and on and on, but there are some complete reasons why we agree or disagree with this argument. (D class, May 9)

As the teacher was attempting to move the discussion forward by offering a kind of summary, one student took the opportunity to challenge the task question altogether. The teacher then challenged the student to improve the question.

Mr. Doi: $\quad$ It's an eternal subject of research, if you're interested in linguistics, I encourage you to look into this. If you solve this puzzle, I think you can get the Nobel Prize.

Student: But can you solve it? This question itself is really vague, I think. I mean-

Mr. Doi: $\quad$ Sure.

Student: It doesn't say like, you know, it doesn't give us any specific examples, and if-you gotta bring in some other authors... when I think of someone else's ideas that's missing, you know, the question doesn't work.

Mr. Doi: Good. So... it's up to you to deal with this question. (D class, May 9)

The discussion finally culminated in one student's emotional resistance, shown in the following transcript. Although Mr. Doi did not exploit this point, it had become clear that having a strong opinion was an advantage to developing ideas. 
Mr. Doi: $\quad$ This is the idea... and the Japanese go around, never getting to the point. But English speakers, get to the point. Maybe so... When I speak in Japanese, I may go like this. When I speak in English, maybe I go like this. Who knows...

Student: Who said that?!

Mr. Doi: Who said that? I don't remember... [students laugh] I guess cultural anthropologists... we'll continue to watch that... (D class, May 9)

Student participants in the A and B classes often expressed dissatisfaction with the materials in the safety of the interviews but rarely in class. In one class however, one student's comment was missed while the other's was picked up by the teacher, and quickly given an absolute response:

Student: I hate this...

[Then, nearby]

Student: $\quad[$ softly] This is hard...

Teacher: This is hard, it's not easy. Coming up with a thesis sentence is not easy! (A class, October 10)

In that same class the observation recording also picked up a private conversation between student participant Megumi and her friend expressing discontent:

Megumi: $\quad$ I'm never going to be applying for business school...

Student: Don't think about it. We're just doing it for class.

Megumi: And I don't think I'm going to be making a web page either.

Student: I know. There are other people to do it.

Megumi: $\quad$ Use professionals! (A class, October 10)

In the C class, students were not observed verbally critiquing the content or materials in class. However, in her early interviews, when asked why she was observed playing with her hair and looking tired and bored in class, Yui expressed dissatisfaction. She complained: 
It's way too slow and way too easy I think. And it's not only me... I wanna write more - not just paragraphs - I want him to give us a topic and we write more and then make the whole essay better. (June 21)

\subsubsection{Summary}

From these examples it seems that teachers' cultural expectations of EFL writing may have been what led them to invite critique of class materials in varying degrees. In one class (D) the students challenged the materials as requested by the teacher, and this continued throughout the course initiated by the students. In the distinctly skills and genre focused discourse classes, students either murmured discontent during class and then "vented"

during interviews (A, B, and E), or seemed to openly accept or at least eventually "give in" to the teacher's specific focus of the course (C). What was most significant about all of these cases was that it was only in the D class where the teacher showed the students the importance of being critical through the example of critique of the course materials and content.

\subsection{Chapter summary}

A student's personal ideas about a topic may or may not get to be developed in an academic writing class, but with the abundance of time in many of the observed classes spent on considering the audience in making thesis statements, it seemed inevitable that discussions on the development of those thesis statements would also be observed. A number of themes emerged from the data in regard to discussion of theses, including: brainstorming; establishing a position or claim; and use of personal experience as supporting evidence.

The most common of these by far was brainstorming, observed in every class. Interviews, however, revealed that some students, though appreciative of the inherent value of brainstorming, did not always find it useful, particularly when the guidelines were seen as too strict. One student (Aki, discussed end of 7.1.1) expressed relief at not having to do the brainstorming step in the writing process beyond the immediate class.

In terms of establishing a position or claim, observation data suggested that this was a particular area where students developed their writing skills to a greater extent. In their 
interviews, although many students felt confused by this, others felt it was the most valuable of the writing skills worked on in the course.

As for the use of personal experience in academic writing, much like the features discussed in the previous chapter, there were large discrepancies between teachers' approaches. While Mr. Clark encouraged it for use as supporting evidence, other teachers (Mr. Doi and Ms. Ellis) used it only in a discussion of developing the thesis. In these discussions the issue of topic familiarity was addressed, but not dealt with-a distinct problem according to Stapleton (2001) for students trying to improve their writing skills through the use of critical thinking.

In addition to these themes, the final point was used as a way to collect all the observed instances of students actively expressing their own ideas and opinions on the course materials and/or content. This was significant in terms of actualizing many of the comments students and teachers provided in interviews in terms of how they felt the course materials actually helped them develop their own ideas in their writing.

The next chapter provides an analysis of a selection of the students' written texts. The analysis includes considerations raised by the analysis of the observation data as the input to the students' output in their writing. 


\section{Chapter 8. Writing with multiple selves: Analysis of students' written texts}

This chapter is first divided into two main parts: writer identities and writing game strategies. As described in the methodology chapter, the analysis of the students' written texts was done using a framework based on Appraisal Theory within Systemic Functional Linguistics (Martin, 1997; 2000), adapted using Bakhtin (1986). Appraisal Theory is a framework that assists in the "analyses of stance as positioning in relation to values and voices in a text" (Hood, 2004, p. 13). Martin (2000) emphasizes that this framework provides a system for linking concepts to areas of meaning making, such as the concept of ENGAGEMENT, which indicates a communicator's "degree of commitment to the appraisal being expressed" (Hunston \& Thompson, 2000, p.142). Furthermore the system examines the way communicators "exploit different ranges of appraisal to construct particular personae for themselves" (Hunston \& Thompson, 2000, p.143).

The analysis of the data follows Martin's $(1997 ; 2000)$ Appraisal Theory systems including ATTITUDE, ENGAGEMENT, and GRADUATION, with adaptations to subtypes based on ideas from Bakhtin (1986) and White's (2005, p.2) suggested ENGAGEMENT subtypes. The adapted framework provides a specific focus of the writer's attitudinal positioning in and "degree of commitment" to the text as well as the construction of "particular personae." The adaptation also includes an analysis of which selves were used by the student participants in establishing their writer identities (Clark \& Ivanič, 1997). As discussed in section 4.3.3, the students' language choices in their writing indicated which selves they displayed. In the system of ATTITUDE, depending on the content and context of their language choices, displayed an authorial or, in the case of personal experience, an autobiographical self. In the system of ENGAGEMENT, students attributing ideas to outside sources showed a discoursal self, while the other aspects of ENGAGEMENT further supported an authorial self. In addition, students using language of GRADUATION such as Force further displayed an authorial self.

It is the three identities-discoursal, authorial, and autobiographical-that make up the subsections for the first part on writer identities. For the second part, I will provide an 
analysis of which writing game strategies were used (Casanave, 2003) in the selection. Each strategy will make up one subsection for that part.

After the explanation of these two main parts, I then provide a detailed analysis of four students as case studies. In the analysis I provide a brief student profile, including information about their beliefs about writing, expectations of the course, impressions of their teacher, and a description of the specific writing task selected for the analysis. This is followed by a closer look at the students' writer identities, writing game strategies and language use as identified by the preset indicators within the Appraisal framework. In this more detailed analysis I also discuss the implications of the points raised in section 2.2.1 regarding deductive and inductive writing, shared cultural knowledge between readers and writers, and whether or not students mimicked features from their sources or established their own voices.

\subsection{Self (Writer Identity)}

As discussed in section 4.3.3.2, the idea of a possibility of various selves or writer identities in writing is part of Halliday's (1985) concept of "interpersonal meaning," including the ideational meaning-the topic being communicated-and interpersonal meaning-how the writers position themselves in relation to the discourse (Fairclough, 1992; Ivanič, 1994). This is building on Martin's (2000) emphasis that attitude in writing is interpersonal, as it is how the writer attempts to connect with the reader (Hunston \& Thompson, 2000).

As discussed in section 2.2.2, Hyland (2002b) explains that students of academic English composition are often required to follow specific style guidelines prescribed by their instructors, and this may include a particular requirement for students to write objectively and essentially remove any personal identification from the content of their writing. This is often done in an attempt to get students to take a more academic-and less emotional or personally motivated-approach to the argumentation in their writing by relying more on source evidence rather than personal experience. This point is contentious however as university instructors, even in the same program on the same courses, do not always agree that this type of requirement is necessary or helpful. 
The following features of writer identity and Appraisal Theory were used in the analysis and served as codes for the data, the three selves serving as the subsections for this part of the chapter:

1) Self, including

a. discoursal - objective with any overt sense of self absent from text;

b. authorial - self is present in establishing that writer has something to say; and

c. autobiographical - personal experience is the focus of the writing and/or main evidence for support;

2) ATTITUDE, used to establish authorial and/or autobiographical selves, including

a. Judgment - value of statement made (positive or negative) in relation to human behavior, necessary for establishing and developing a thesis;

b. Appreciation - value of statement made (positive or negative) in relation to objects, products or processes, also necessary for establishing and developing a thesis; and

c. Affect-emotional/affectual response;

3) ENGAGEMENT, used to establish a discoursal self while developing one's thesis, including

a. Modality - probability (could, may, might, etc.) and obligation (should, etc.);

b. Reality phase - it seems, it can be concluded, etc.;

c. Attribution - credit given to source or hearsay;

d. Proclamation - In fact, It is true, etc.;

e. Expectation - of course, etc.;

f. Counter-expectation - surprisingly, etc.;

4) GRADUATION, including

a. Force - slightly, very, surely, obviously, etc.; and

b. Focus - effectively, truly, etc.

\subsubsection{Discoursal self}

The discoursal self was most often how student writers attempted-not often successfully-to represent themselves in their writing when taking an objective stance on 
their arguments. The idea of the discoursal self is that the writer attributes ideas to outside sources or other authorities, developing the writer's voice as s/he wants to be heard, as opposed to developing a voice through the stance taken. Students of Mr. Doi (Composition 2) and Ms. Ellis (Composition 1 and 2) were taught that being objective and impersonal would make their arguments more persuasive, while Mr. Clark (Composition 1) made no mention of writing persuasively, but did instruct that using certain personal pronouns would make the students' writing stronger. Ms. Aiba (Composition 2) did not give assignments that concerned elements of the self, yet the students did use different voices for each of their writing tasks, as appropriate to the purpose of the task.

Much of the dilemma for students using the discoursal self is that, as novice writers, they do not have the level of exposure or experience required to present themselves accurately in the desired discourse (in this case, argumentative essays) or to successfully attribute their ideas to other authorities. Schneider and Andre (2007) explain, "Students may convey their lack of identification with academic discourse through their misuse of citation conventions or specialized terminology or through their failed attempts at employing complex sentence structures in order to sound more academic" (para.5). In the classes where teachers required students to essentially take on a discoursal self in their writing (D, $\mathrm{E}$ and F), this seemed to be where students struggled the most in terms of dealing with their thesis, and in dealing with the academic writing aspects of their writing task. It seemed they were overwhelmed by the assumed expectation of the teacher and their lack of experience required in order to meet those expectations. The result was ultimately mixed, as it seemed to depend on just how familiar the chosen topic was to the student-a point established by Stapleton (2001).

In the E class, there were dilemmas. All students were encouraged by their teacher to write objectively, but to choose argumentative topics that they had strong opinions about. Nana's approach in her paper on the jury system recently implemented in Japan managed to use language that resembled something closer to a discoursal self, although she did use the pronoun our twice, referring to Japanese people. Although a certain amount of Affect was 
used in her paper, the phrases used to develop her anti-jury system thesis were relatively un-emotive, including:

should not be enforced;

disadvantages of this new system are beyond its advantages;

the trials will lose accuracy;

trials will be places of more emotional arguments;

juries would be easily moved;

jurors are more likely to have prejudice;

people generally do not notice;

trials will be inaccurate;

trials will be more concerned; and

the jurors...have responsibility.

This suggests that Nana was not seeking an emotional response from her audience, but rather she positioned herself as an unmovable, confrontational authority that was not open to dialogue with the dissenting voices of other authors.

For Yuki, her paper on adventure sport was focused very much on her sources, in particular a documentary that promoted adventure sport. Yuki used significantly more sources (26) in comparison to the other students-well beyond the task requirements. In doing so, there was a large number of attributive phrases, and in fact, every other aspect of ENGAGEMENT was used in varying degrees, signifying a fairly successful attempt at displaying a discoursal self. The attributive phrases included:

according to Lowenstein;

many people believe;

Supporting this, Oliver;

as an example, Krein;

in his study, Breivik;

Malkin (1998) also observed;

This is expressed in Joe \& Simon's comment;

held by Scouts Canada; 
In...No fear: Base jumping;

Self et. al (2007) examined;

Simon's remark;

The issue with Yuki's paper was that in the emphasis of her pro-adventure sport thesis, she used a very large number of value judgment phrases, i.e. assessments of human behavior (White, 2005). Although the presence of the high number of attributive phrases in Yuki's paper would normally indicate a discoursal self, it is evident from the even higher number of Judgment and Force phrases that Yuki was displaying a primarily authorial self.

Perhaps the most successful student to display a discoursal self was in the D class. Satoko chose to write on the topic of socialized medicine, focusing on the situation in the US. Satoko had spent a number of years in the US and had done work on this topic in her classes there. Also fortunately for Satoko, her composition teacher was an American history scholar, and was able to provide insightful feedback on her drafts. Satoko chose to focus her thesis on the benefits of socialized medicine by pointing out that the US is the only wealthy, industrialized nation that does not provide universal health care. Since Japan does provide a form of socialized medicine, she used that as an example of a system that worked, and one that the US could adopt. It was a simple approach, one that seemed to work in persuading her class and her teacher that she was an A student.

In the Composition 1 classes both of which required students to write argumentative essays, it was discovered that regardless of any of the teachers' requirements regarding writer identity, there was still a slight variation of identities used by the participants in the study. In the F class, the four student participants wrote argumentative essays in which they were instructed to write objectively. In their attempt to meet this task requirement, three of the four students avoided all personal pronouns, and all four used Attribution for their sources and attempted to keep emotive language to a minimum-with varying success. In addition to the Attribution phrases, they also used a variety of other ENGAGEMENT phrases, such as reality phases including it seems, proclamations such as it is/is not true, and expectations including probably and it can be expected. 
In the F class, Rika's anti-animal testing and Hideo's anti-teenage-cell-phone-use theses were both fairly well attributed, although the number of sources (Rika 6, Hideo 3) was minimal, and the reliability of the sources was not a concern, as that aspect had not been required for the task. The number of attributive phrases used by Rika and Hideo displayed an attempt at a discoursal self. Akiko's pro-capital punishment paper had the greatest number of attributive phrases even though none of her sources were actually cited (Akiko confirmed in her interview on January 24 that she never understood how in-text citations actually worked), but was filled with value judgment language such as:

falsely accused people will suffer;

the crime victim families would not be satisfied; and

heinous criminals,

among others, showing a more authorial than discoursal self. But of the four, it was Chinami's paper that was most striking since it included the pronouns we and you, even though the teacher specifically forbid the students to use them. Also, Chinami used a number of sources (6) and accordingly a number of attributive phrases. Other ENGAGEMENT aspects were used such as Modality and a Reality phase, which showed a fairly strong subjective authorial self added to her use of a number of value judgments.

In the $\mathrm{C}$ class, one of the three student participants, Aki, used what appeared to be at times a discoursal self, attributing her ideas to outside sources. But quite obviously against this analysis, she did use the pronouns we and you in her essay.

Ultimately, although these students attempted to use the discoursal self in order to meet the expectations of their teachers, in developing their theses and attempting to persuade their readers, most students ended up with a more authorial self.

\subsubsection{Authorial self}

The authorial self is generally used in situations where student writers assert their opinion on a topic through:

- evaluation (usually of source materials - showing their position on the topic in relation to those of other writers),

- the use of modality in and qualifying of ideas, 
- through particular use of attributive tags, or

- through authority derived from their experience or awareness as readers (Clark \& Ivanič, 1997, p. 152).

Use of the authorial self in the students' writing was most apparent in the utilization of personal pronouns such as we and you. This is not specifically the authorial self Clark \& Ivanič (1997) described, but it does suggest that these students are positioning themselves in relation to authorities on their subjects, and that they have something to say. The authorial self refers to the textual 'evidence of writers' feeling of authoritativeness and sense of themselves as authors" (Clark \& Ivanič, 1997, p. 152).

For the analysis of students' writing in the A and B classes, since they did not write any argumentative essays, I chose their task of writing a "response letter to the editor" on the topic of 23-year-old Japanese traveler Satoshi Nakamura's kidnapping by terrorists in south-east Iran on October 7, 2007. For this task a sense of authority was required, suggesting that an authorial self might be present in their writing for the task. Of the four students who did this task only one student, Megumi, attempted to use the authorial self, although in doing so revealed weakness in the attempt to present herself as a political commentator-ultimately the self she would have needed to establish if it were to be truly authorial. Megumi did not use any personal pronouns, but there was still a clear personal stance taken on the subject matter. For example, some Affect language used included:

it is upsetting;

outrageousness;

selfishly;

terrible; and

lack of sense.

However, use of the Reality phase sounds as if, the Counter-expectation it is surprising, and a number of cases of GRADUATION suggest that Megumi did have some understanding of the qualities of political commentary. When interviewed about it, Megumi explained that she wanted to write persuasively, and believed the best way to do that was from a collective Japanese position: 
Jim: $\quad$ You used this quite emotional language that is appropriate for things like response letters to editors, and the thing that's interesting to me is that you didn't personalize any of it. Can you explain why you did that?

Megumi: $\quad$ Yeah, like, I thought every Japanese should feel the same way as I thought. That's why I wrote it that way.

Jim: $\quad$ Ok, so you wrote it from the perspective of all Japanese people. Why didn't you just say, "I was upset", "I feel this way", or "I think Japanese people should feel this way"?

Megumi: Because this thing that happened in Iran was unfair. It didn't have to be him. How the Iranian government is treating the situation is like not proper...

Jim: $\quad$ ok, so it goes against sensibility...?

Megumi: I thought we should have like common feeling. (December 11) Megumi's language choices in universalizing her own sentiment in her attempt at political commentary indicated an authorial-autobiographical self that was based too much on assumption and relied too much on emotional appeal.

In the E class, although the features of a discoursal self were apparent in an attempt to write more objectively as instructed by the teacher, elements of an authorial self were present in each of the students' texts. These were longer research papers of around ten pages or 2000-2500 words, similar to those in the D class. In Satoko's paper on universal health care, she expressed some authority in addressing a significant counterargument to her thesis. She wrote:

There are many people who will be able to gain the benefit of universal health care in America since more than half of population does not have health insurance. Many people would be able to go to see the doctor without having to worry about the cost of the bill. However, some insist that it would be difficult at the moment for America to change the health care system because changing the health coverage from private to universal will add strain to the current economic situation. This argument ignores the fact that America currently spends more money than the other countries in the current 
health care system and as the expense is increasing more as the years go by, it is making it harder for America to change the system.

It was noted in all of the papers in the D and E classes that students successfully utilized an authorial voice in their thesis and conclusions-an indication that they all considered their readers and recognized the persuasive effect of the authorial voice. In Yuki's paper, the emphasis of her pro-adventure sport thesis was done using a number of Judgment and Appreciation phrases such as:

these activities... are not absurd;

they are...rational;

solid principles;

usually hold judicious and acceptable motivations;

they have profound and autonomous personality;

more dangerous and challenging;

risk is...subjective;

the experience will result in disaster; and is not a foolish activity,

among others. These were supported by a number of Force (GRADUATION) phrases including:

actually (x3);

it is obvious that;

even ( $x 3)$;

completely;

very ( $\mathrm{x} 2)$;

incredibly;

tremendously;

apparently;

only;

extremely; and

just. 
Although Yuki had attributed most of her supporting ideas to outside sources, indicative of a discoursal self, she had chosen to evaluate the ideas from those sources, adding value judgments of them, clearly displaying an authorial self.

In the A and B classes, the students were given the task of writing a response letter to an article on a Japanese university student taken hostage in Iran. Three of the four students appeared to use an authorial self in their letters (while one student used a mix of authorial and discoursal selves), judging clearly whether or not they believed the Japanese government should or should not negotiate with the terrorists for Nakamura's release. However, students used a number of personal, emotional phrases based on personal context as well, having the effect of reducing their authoritativeness and indicating an autobiographical self was present. But all four also attributed the article (provided by their teacher) in order to focus their critiques, and used various terms of Force (Fumiko: certainly, Miki: obviously, etc.) to emphasize their authority.

In the $\mathrm{C}$ class, the students were not required to use any outside sources to support their thesis. They were required to choose from a short list of argumentative topics and to use what they knew to support their ideas. Two of the three participants chose global warming while the other chose smoking. Both students who wrote on global warming used an authorial self, addressing the reader directly with the pronoun you, and using the pronoun $w e$ in an attempt to strengthen the argument. However, a distinct difference was that Yui did not choose to attribute any source of any kind in support of her thesis, while Aki referred to "TV news and newspapers" and also provided examples such as The Kyoto Protocol and the Toyota Prius. This suggests there was reliance on the sources to support and develop her thesis. In her interview, Aki commented:

This wasn't first time for me using references and citations. I thought they really help to make my writing more persuasive. It wasn't just "I think this"... (Aki, January 24)

Yui, on the other hand, used Reality phases in order to engage her reader and took a more direct, personal approach in encouraging her readers to agree with her thesis. In her interview, she commented: 
I was just doing what [teacher] wanted us to do. Since we didn't have to use sources, I didn't use any. When he said "persuade your reader" I just used common sense for my argument. (Yui, January 22)

Saki was the only one of the three participants in the $\mathrm{C}$ class to use the ENGAGEMENT aspects of Modality (can be; it is possible), Proclamation (as it is well known; it cannot be denied; it is true) and Expectation (of course), and along with her Judgment phrases and overuse of Force phrases clearly displays an authorial self. Like Yui, Saki also did not attribute any sources, and in her follow-up interview made similar comments in support of the decision.

Ultimately, the authorial self was the most prevalent in the students' writing, especially in the presentation of supporting evidence-either from sources or personal beliefs or common sense-in developing the thesis. Students instructed to be objective in their writing did so only to the extent that discussing the argument allowed them to. When it came to developing the thesis, the voice of the writer was clearly authorial. The remaining area in this analysis was of the autobiographical self, since students were for the most part instructed not to use any personal evidence.

\subsubsection{Autobiographical self}

The autobiographical self is used in students' writing when personal experience is the topic-such as in personal essays or personal narratives-but generally not used in argumentative academic writing. This means that for this study, nearly all students used the discoursal and/or authorial selves in their writing. However, in the A and B classes the students did not write argumentative essays, and the selected writing task of a letter to the editor revealed that two of the students displayed in addition to an authorial self certain Affect phrases of personal nature that indicated an autobiographical self was present. The phrases were used as a sympathetic rhetorical mode (Megumi: it is upsetting, Hiromi: it is unnerving, etc.)

In Composition 1, in the $\mathrm{C}$ class, there were no specific instructions given on what types of evidence students should use other than that no research was necessary for any of the papers in the course. This instruction was for students to use their own personal

experience as supporting evidence, which the teacher believed was a motivating factor for 
the students to write. In their papers, students displayed mostly an authorial self, using a number of Judgment and Force phrases. As the evidence presented was mostly personal, the autobiographical self was certainly present in Yui's and Saki's papers, as they did not use any outside sources, but less so in Aki's paper as she did use outside sources. Interestingly, only Saki used Affect in her short essay. With a thorough analysis of the content and context of her uses of ATTITUDE language, it was clear she used an autobiographical self. In their interviews, the student participants in the C class expressed satisfaction with the opportunity to write about their own experiences, in support of the teacher's philosophy.

In Yui's paper on preventing global warming, all her evidence was based on personal experience. For example, her first main idea-stop wasting energy-was supported by activities relevant to her own life including:

turn[ing] off the lights when leaving a room, not letting water run when washing your face or brushing your teeth, and not setting the air conditioner at an excessive temperature.

Her second main idea-minimizing trash-included refusing plastic bags or disposable chopsticks usually provided with purchases at convenience stores in Japan. Aki's paper on stopping global warming was contextualized in Japan, although she did not mention Japan explicitly. Her supporting evidence was mostly from outside sources, but on her main idea of recycling, she used personal experience in support, including: having a flea market (a common event in Japan) as a good way to reuse old things. Saki's paper on tobacco and nonsmokers described situations specific to living in Japan, though Japan was not mentioned. Supporting evidence was all based on personal experience. Her first main idea-separating smoking and nonsmoking areas-was supported by the example of poorly partitioned restaurants-common in Japan.

\subsubsection{Summary}

In every case, the selves displayed in the students' writing corresponded with their attempts to meet the requirements of the task. In the D, E and F classes, in argumentative writing tasks where teachers required students to cite sources to support their ideas, every 
student displayed an authorial-discoursal self, mixing Attribution to outside sources usually with a high number of Judgment phrases. In the C class, where students were not advised to use any outside sources, two students displayed an authorial-autobiographical self, using personal experience to support the thesis, while the one student who used outside sources in an attempt to make her writing more persuasive, displayed an authorialdiscoursal self. In the A and B classes, students in their letters to the editor all displayed an authorial self, with two students showing more of an authorial-autobiographical self through the use of emotional language of personal context.

For these Japanese university students, the issues related to writer identity lie in the attempt to meet the expectations of the teacher while at the same time trying to establish and develop a thesis. Teachers encouraged their students to write objectively, which Hyland (2002b) suggests is problematic, since many students have not learned appropriate strategies for writing objectively. In the classes observed in this study, the students were taught the importance of the thesis. They were expected to establish and develop the thesis with a variety of strategies (depending on the class) without discussion of maintaining objectivity. Students ultimately used, for the most part, a relatively subjective authorial self in their attempt to meet their teachers' expectations.

\subsection{Writing Game Strategies}

Casanave's (2002) Writing Game Strategies outlines six different approaches student writers take in meeting the expectations of writing tasks. These strategies include (pp. 6174):

1) Interaction with texts: Does the writer engage in conversation with authors? Do they challenge or evaluate authors?

2) Blending voices: Does the writer merge her/his own voice with authors'? Does s/he simply take the authors' voices? Does s/he fail to bring authors' voices into the text?

3) Owning research experiences and telling a good story from them: Does the writer manage to present her or himself in the text through a high level of familiarity with the subject matter? 
4) Speaking with authority: Does the writer take a strong critical stance on the subject matter?

5) Learning to love writing: Does the writer show a level of fluency relative to the readings? Is it a long piece of writing?

6) Making the paper look right: Does the text fit the prescribed organization and structure as developed in their writing classes?

The first two strategies are relevant only to the students' writing that incorporated outside sources as supporting evidence and interacted with their peers and teachers in discussions of them. The third strategy involves a certain level of personal involvement and experience with the writing topic-something students attempted to take advantage of only to a certain extent. The fourth strategy requires students to establish and develop a strong opinion on their topic, corresponding with an authorial self. The fifth strategy shows a "love of writing" through fluency and comfort with the topic. The sixth strategy fits in with students' attempts to meet their teachers' expectations of their writing task.

Upon first considering using the writing game strategies as an analytical framework, I had anticipated that I would surely discover notable connections between the strategies used and the writer identity established. However, this was not necessarily the case.

\subsubsection{Game strategy 1: Interacting with texts and with others about texts}

For the first strategy, the students in the D class stood out as significant cases where students evaluated or discussed their texts (although peer reading and teacher feedback and consultations were implemented to an extent in the C, E and F classes). Students used outside sources to support and develop their thesis but did not challenge them or negotiate with others about using them.

In the D class, although the students were to some extent encouraged to evaluate their sources, that evaluation was lacking in their writing. Where the evaluation did occur was in the regular peer evaluations and reader response tasks. For the second half of the semester students in the D class were divided into three "research groups" of 6-7 students-grouped 
together according to geographic focus of their chosen topics, namely: Japan-related, USrelated (including Satoko), and other (including Aya). Each group spent time together reading each other's papers and offering feedback. Later, each member of the group had to present their paper to their group plus another group. Each student was assigned a primary peer reader who was responsible for helping her/him to prepare the presentation and offer evaluation after the presentation. All other students were required to offer some written feedback of the presentation.

The students responded positively to this organization of the class. However, in their interviews, Satoko and Aya took different positions on this. Though Satoko was very positive about this organization, she felt it was due to her grouping with other strong students who were willing and able to provide constructive feedback. Since the students' topics fell into the teacher's research area, Satoko felt positive about the interaction she had with her teacher as well. Conversely, Aya felt her group was relatively weak and she had hoped for more interaction with her teacher, rather than the group. Because Aya's group's topics were outside the teacher's area of expertise, those students were required to consult with other professors at the university with expertise in their specific topic areas. Aya met with a woman who taught Islamic studies at Midori, but felt she had not prepared properly to make her interactions with the Islamic studies professor valuable.

Although all the other classes included small group discussion activities in class, these discussions ultimately did not play a significant role in the writing process. For the C, E and F classes, students participated in peer reading activities, but in their interviews, they indicated that as much as they appreciated and enjoyed the opportunity to read each other's papers, they did not feel the activity had any impact on their actual written texts. In the A and B classes, students were grouped for the final group project, but the discussions in these groups were conceptual, and focused more on division of tasks, rather than collaborative writing.

\subsubsection{Game strategy 2: Blending voices}

The second game strategy seemed to be a particular struggle for the student writers in this study as it required them to combine their own voice with the voices of the authors of their 
outside sources. For the students in the D, E and F classes this was a pertinent issue as they were required by their teachers to cite their sources for every summary, paraphrase and quote. The students in the A and B classes were not required to cite any sources for their writing tasks as the genres of writing they were practicing did not necessarily require them. The students in the $\mathrm{C}$ class were given no requirement or instruction on doing citations. Because of this the one student who did use outside sources did not clearly cite her sources, but instead referred to them without any particular referencing convention. There was no borrowing of other voices in this practice.

For the students who were expected to blend their voices with those of their sources, the issue boiled down to whether or not they were using their outside sources to develop an argument they established themselves, or if they were borrowing the argument from their sources. In the analysis of the arguments used by the students in the D, E and F classes, there was a noted trend that students in Composition 2 (D and E classes) were more likely to establish arguments of their own, while the students in Composition 1 (F class) appeared to borrow their arguments from their sources.

In the D class, Satoko struggled with the concept that her voice was blending with the voices of other authors, as this was the first time for her to consider this concept. Although she had used outside sources and learned how to cite them in earlier classes, she had not attempted to evaluate the sources, but rather simply use them to provide factual statements in support of her thesis. With her research paper on universal health care, Satoko did introduce information from some sources, but did not display clear understanding of how that information compared to other source information as well as her own thesis. Although her sources remained unevaluated, Satoko did show how different ideas in her sources either supported or rejected her thesis.

Also in the D class, Aya's blending of her voice with the voices of her sources resulted in a somewhat convoluted development of her thesis. The feedback she received from her peers and teacher on her introduction was that it provided only a vague, abstract background to what it was she really wanted to say. This was likely the result of trying to make her thesis fit in with what her research group and the rest of the class thought she should do with it, 
even though she disagreed. Aya had initially wanted to insist that Shabina Begum should have been given the right to wear her jilbab in addition to her school uniform, but she had no support from her audience. Her main supporting sources focused on human rights, while her opposition, including her opposing sources and her audience, maintained that the issue was a matter of authority of social rules. Ultimately Aya's voice seemed to be lost in a wave of emotional appeal that never seemed to take advantage of the deeper argument made by her supporting sources.

In the E class, all three students' papers showed a great deal of Attribution along with a high count of ATTITUDE phrases. In the moments of clear and successful Attribution, the blending of voices seemed to go fairly smoothly. For example, Nana struggled with somewhat awkward Judgment phrases including disadvantages of this new system are beyond its advantages and the trials will lose accuracy, but did show some successful voice blending by using Judgment phrases with her supporting sources.

In Akiko's (F class) paper on capital punishment, the cases of Attribution varied from:

some people believe, to

a poll by Sankei news, to

according to Asahi newspaper

Her assertions remained strong with a number of ATTITUDE phrases. Rather than blending the voices of other sources, Akiko instead highlighted the journalistic neutrality in stark contrast to her strong opinions. Akiko's classmate Hideo took a similar approach with his paper on children's cell phones. He used relatively weak Attribution, ranging from according to the survey to the statistics revealed, that was overwhelmed by his overuse of Judgment phrases, describing, in his own voice, children's cell phones as:

luxurious,

exclusive tool,

ubiquitous device,

invite them to dependency, and

involve them in cyber bullying, and more.

The voices of his other sources were not utilized. 
In the same class, Chinami's paper on teen pregnancy used a variety of reasonably reliable sources, and while Chinami asserted her own voice through extensive use of Judgment phrases, it was clear that the voice in her claims was adapted from her well-selected sources.

This game strategy was a challenge since students' mimicking of the voices of their sources would occasionally result in mild cases of plagiarism noted in feedback by the teachers, especially Ms. Ellis. Students in the D, E and F classes practiced with summarizing, paraphrasing and quoting, but as Ms. Ellis explained in an interview, for most students this was their first time with this writing skill, so they just needed to keep revising.

\subsubsection{Game strategy 3: Owning research experiences and telling a good story from them}

This strategy requires students to do some in-depth research on their topics in writing research papers as opposed to "library papers" or term papers. For the most part, the kind of research students conducted in the process of writing their papers was not in-depth, but rather more introductory or informative with some minor analysis conducted in the form of interpretation or argument. For example, students in the $\mathrm{C}$ class were not required to write in-depth research papers as the teacher in that class explained in his January interview that many of the students in that class were learning basic essay construction for the first time. An issue that did arise in the $\mathrm{C}$ class however was one of a lack of ownership of writing due to strict instructions on the writing tasks, discussed in section 8.2.6 below.

Expectations in the $\mathrm{E}$ and $\mathrm{F}$ classes were higher than the $\mathrm{C}$ class. These students were required to write library-based research papers. However the focus was much less on the research and more on essay construction for the purposes of developing a thesis. The teacher of those classes explained in her January interview that the students needed to learn the basics of an essay including introduction, body and conclusion, and that introducing and citing sources in the essay was at the introductory level for them so she did not expect much beyond that. The students in the E class explained that they learned the importance of such practices as refuting an argument for the first time. 
In the A and B classes, although students did some field research by interviewing students and university administration for their final group project, there was no research paper produced. However, the students in the A and B classes all described a certain level of ownership of their contributions to their group's webpage, which was important since the groups were required to present their webpage to the class. The class then voted on the best webpage and the winning entry was actually posted to the Midori University website.

Students in the D class were required to discuss their research with an academic or expert on their topic. This led to some ownership of the research topics, but ultimately this was strongly affected by the background and expertise of the student writers themselves. Since Satoko had lived in the US and her teacher was her expert for her topic, she was able to display fairly strong ownership of her writing. For Aya however, her limited background knowledge of the topic, the attempt to assert her own personal feelings on the topic without any strong data to back it up, and the two superficial interviews she held with her topic expert, resulted in a distinct lack of ownership of her writing. In fact there was an obvious gap in what she had intended to do and what she actually wrote. She knew she had to use her research data to support her thesis, not just her own personal feelings. Because she did not have a strong grasp of the research data, the feedback she received from her teacher indicated that she was unable to use it fluidly or effectively.

\subsubsection{Game strategy 4: Speaking with authority}

In establishing and developing a thesis, it was clear that for all of the students in this study, speaking with authority was a useful strategy. Casanave (2002, p.69) explains, "The practice that novice readers and writers engage in influence how they identify themselves within their academic settings and the authority with which they learn to express their knowledge and their identities." One reason all the students in the current study utilized this writing strategy may be that they had been taught that writing in English is deductive not inductive. This is different from writing in Japanese, in that in English academic writing the thesis is overtly stated in the introduction, requiring the writer to take an authoritative stance. Whether students attributed the main idea to a source or made the stand on their own authority, this is what Ivanič (1998) described as the self as author, or authorial self. 
Taking a stand from the beginning instead of the end of the essay was one of the consistent points of contrastive rhetoric noted in most classes (less so in the A and B classes). This act of stating the position in the introduction is understood as an act of speaking with authority, according to the students in their follow-up interviews:

I stated that the Japanese government should stop negotiating with the abductees in Iran, that's the thesis statement, and that was my opinion that I made on my own. (Megumi, A class, January 23)

Last year [in Composition 1] I thought thesis statement should be not so specific. But this year [in Composition 2] I think maybe thesis statement should be very specific because I have to state strong opinion at the beginning, not the end. (Yuki, E class, January 23)

Casanave (2002) notes that in her research, students could manage to speak with authority even in less conventional writing tasks. In the A and B classes where students did not have traditional academic essay writing tasks, they were expected by their teacher to speak with authority. The writing tasks of a book review, response letter to the editor, and a promotional webpage all necessitated the students' ability to take strong, authoritative positions and maintain and develop those positions throughout their texts. Expressing opinions did not seem to present any particular obstacle for the students; the issue was rather that the students were unsure of the strength of the opinion. For example, Megumi commented:

Megumi: Well it wasn't difficult expressing my opinions...

Jim: Ok.

Megumi: But I didn't know if it was good or not. (January 23)

It seemed the greatest challenge of speaking with authority was how to make the student's voice "sound" authoritative. Casanave (2002, p.70) asserts, "students learn to mimic an authorial voice as part of the writing games they become familiar with in college." With the students in this study, the authorial voice was noted particularly in cases where writers presented facts from their sources. In the many examples of Attribution, students relied on the power of the outside source to be the voice of authority, and did not have a sense of how their own voices were affected by that. For example, Satoko (D class) had this to offer: 
... it was hard for me to get academic sources. It was first time to access JSTOR and all those things, because I'd never done it in junior high or high school, so it was really academic for me, it was first time for doing that, and after, er- right before I turned in this paper - or after, I don't remember, but I talked to [teacher]-sensei, and he gave me some advices to how to do the research more mm... professionally? Like, how do I say this - he told me that I was picking the information that I want, so I mean, it's biased by my point of view, and I had to correct that - that's what he said

- so there are some things I that have to, you know - not revise - but, change for the paper... (Satoko, January 22)

Ultimately for this game strategy, students seemed to see it as a necessary part of developing their thesis. It also served as a kind of "default" strategy when the blending voices and owning research strategies could not be achieved.

\subsubsection{Game strategy 5: Learning to love writing}

Learning to love writing is a strategy that involves a student's ability to display a higher level of fluency through a clear, non-hesitant approach that can really only be achieved through building up confidence as a writer. Improving fluency in writing often comes in line with fluency in reading. Students required to do reading tasks for a writing class should be able to build both reading and writing fluency.

As described in the methodology chapter, the students at Midori University take as many as 15 classes in their first and second years, so teachers are limited as to the amount of homework they can assign and expect their students to complete. The students in the A, B, and $\mathrm{C}$ classes were given shorter reading and writing assignments. In their interviews, the students in these classes felt this was acceptable, but they could have done more. The students in the A and B classes described dissatisfaction with their assigned tasks, and a love of writing was not apparent. In the C class, the students were positive in their interviews, though only marginally. They felt there were certain aspects of their writing skills that had improved, but there was no increased love of writing.

Conversely, the students in the D, E and F classes described what they considered fairly heavy workloads, and that this was for many of the students "worth it." In the D class, although Satoko found the work less challenging than Aya, they both thought the research paper required a great effort on their part, and they felt their writing had improved 
through the somewhat rigorous tasks. Aya explained that although she struggled with the assignments, she felt the writing aspect of the assignments was what she enjoyed the most, as it was an opportunity for her to express herself. Satoko displayed a fairly clear grasp of this writing game strategy as she often wrote a lot for each submitted draft, and then would make extensive revisions. The fluency of her writing developed with each subsequent draft.

As an introductory course, the F class required a steep learning curve for the student participants. Students were expected to read multiple model essays (from the textbook) for each writing task, answer questions and discuss them in class. In their interviews, they all expressed appreciation for the heavy workload. However, one student, Chinami struggled more than the others. She explained that she had trouble understanding what she was supposed to do when revising her writing-to do what her teacher wanted, or what she wanted. She enjoyed the initial writing task, but the love of writing would diminish, along with fluency, in each subsequent draft.

The E class was less appreciative of the heavy workload, although they expressed marginal appreciation in the interviews. They felt the assignment expectations were not clear, especially compared to their assignments from another teacher the previous year in Composition 1. Also, they often received the comments and feedback on their drafts after excessive delays, at one point as long as two months for some students. The disgruntled class maintained a somewhat negative attitude toward their teacher, though this did not necessarily show in their writing. Students still improved with each draft, showing signs of increased fluency and critical thinking.

\subsubsection{Game strategy 6: Making the paper look right}

The final game strategy focuses specifically on meeting the expectations of the assignment. Students are expected to write in a variety of genres and/or essay types by learning the features and formats typical of them. Casanave (2002, p.73) explains, "[A]rguments persist about whether we should teach students to conform to or resist formal conventions." This is in line with the fairly strict conventions presented in a variety of approaches by the teachers in expressing their expectations of the writing tasks. The two native English 
teachers (Mr. Clark, Ms. Ellis) were stricter with the expected writing conventions than the two native Japanese teachers (Ms. Aiba, Mr. Doi).

The students in the $\mathrm{C}$ class all confirmed that much of the effort made in completing their tasks was getting them to look right according to what their teacher explained in class. Mr. Clark had very specific expectations, down to the use of certain words in the thesis statement for a given essay task. The teacher formatted the outlines of the essays in fill-inthe-blank style that the students could not alter. This resulted in very similar, formulaic thesis statements in each student's text. For example:

Although these problems from global warming are happening, there are still some things we can do to make this situation better. (Yui)

Although it cannot be denied that smoking is harmful to nonsmokers, there are still some ways in which nonsmokers can live comfortably with smokers. (Saki)

Although the situation is getting worse and worse every year, there are still several ways to save our planet from global warming. (Aki)

Both Aki and Saki explained that they thought the "fill in the blank" style was a very good idea and that it gave them a clear idea of what to do for the task. Yui, who had much more academic writing experience in English than her two classmates, was at first very resistant in early interviews. She felt a distinct lack of ownership of the essay, and explained:

...everybody in the class has the same way of writing sentences and stuff and he says to start the first sentence with this word - if this one was a problem and solution topic. He said start with "although" - everybody's essay will start with "although." I mean, the main idea sentence will be - so I thought that was kind of weird. It's not really your essay - it's all - you have to find a problem and fit into this space and stuff. So the style is - it's already made. (November 13)

However, in a later interview Yui agreed that it was good to learn this style of formatting and that she could now choose to format her essays in this way if she thought future assignments called for it.

Students of Ms. Ellis, in the E and F classes, were given more leeway than those in the $\mathrm{C}$ class, but there were clear guidelines given according to APA writing conventions. For the student participants in the F class, this was the first time for all of them to write this way, 
and the first time they had heard of APA or in-text citations. They expressed appreciation for this in their interviews, but struggled to get the formatting right. For example:

According to the survey done by the Ministry of Education, 95.9\% of the $11^{\text {th }}$ grade students own a cell phone (MEXT, P6, 2008). (Hideo)

As stated in article Sex Educatio [sic], reporter, Kotz of teen pregnancy told that "there is not effective contraception and adequate health services, the use of birth control and prevention of sexually transmitted diseases as known as STD." Also as Gale resource stated "children born to teen mothers are more likely than other children to have low weights, putting them at greater risk of dying as infants." (Chinami)

Much of Ms. Ellis's feedback on the F class papers was related to formatting and grammar, and not on referencing conventions. In the E class, because students had learned APA format the previous year, they felt this aspect of the class was consistent, and they all expressed appreciation in interviews.

For Ms. Aiba's students, their classes were genre-based, requiring that they learn the differences of format and style of each genre including a book review, a response letter to the editor, and a promotional webpage. The students in these classes had limited experiences with writing this way, so struggled to understand the differences in approaches to the different genres. All four student participants in the A and B classes complained about the limited feedback from their teacher, indicating it was not helpful in helping them understand what their teacher wanted.

The different bigger writing tasks in the D class were mostly focused on getting students to structure their essays and integrate source material appropriately. Since this was the first time for Mr. Doi to teach English composition, he decided to involve the students as much as possible in the writing process. For Satoko there was great appreciation for this, and an increased awareness of the expectations of the writing tasks. For Aya however, this confused her as she was not sure if she should be writing to meet the expectations of her class, or of her teacher, which seemed to differ according to the feedback she received. For example, in the outline for her paper that she presented to her group, she identified the purposes of the paper as:

i. To have better understanding of why Muslim women wear veil [sic]. 
ii. To find out what causes huge discussions that are based on cultural and religious differences by analyzing a court case of Shabina Begum as an example.

The feedback from her group dismissed the first purpose, stressing this was not a purpose of her paper at all, and suggested the second purpose needed to take a side on the Shabina Begum court case in order to provide a perspective on the discussions. They went as far as to choose the side Aya should be on, which Aya disagreed with. The feedback from her teacher mostly supported her group's feedback on the first purpose, but differed from the group's feedback on the second purpose. Mr. Doi instead suggested that Aya could "use the Shabina Begum case to provide a more specific focus" on the large topic of cultural and religious differences. Aya commented in an interview that she did not understand this feedback, and so decided to use the feedback from her peers.

This writing game strategy was used more in the classes where the student writers were able to discuss their writing with more expert players-either the teacher or other more experienced student writers in their class.

\subsubsection{Summary}

Casanave presented these six writing game strategies as a way to explain what approaches writers at various levels of higher education are expected to understand and use in order to maximize their writing skill potential. She used her experience with writers of both English and Japanese at a Japanese university to make these distinctions, therefore it seemed most beneficial to this study to make use of the writing game strategies as a framework for analysis. It was noted that students who were given more challenging research writing tasks had more opportunities to face all six writing game strategies, and consequently develop as critical thinkers. These were the students in the D and E classes, and to an extent, the $\mathrm{F}$ class. For students in the $\mathrm{A}, \mathrm{B}$ and $\mathrm{C}$ classes, the teachers took a more pragmatic approach to their writing class, which allowed students to get a good grasp of certain writing conventions, but not necessarily to think critically.

\subsection{Case studies}

This section will provide a more detailed description of four students as cases: two students from the Composition 2 classes and two from Composition 1, students of Mr. Doi, 
Ms. Ellis, and Mr. Clark. The only teacher not represented in this group is Ms. Aiba, as there were no argumentative essay tasks in her courses. The student cases are:

Case 1: Aya - D class - research paper on the Shabina Begum case

Case 2: Ai - E class - research paper on sign language education in Japan

Case 3: Rika - F class - essay on animal testing

Case 4: Yui - C class - essay on global warming

These four students were selected based on the following criteria: 1) the student wrote an argumentative paper and gave me a copy of that paper for my analysis; and 2) the student fit into a range of the three different background groups described in chapter 5: one from a traditional Japanese schooling background, one from a traditional Japanese schooling background with one year spent overseas, and two from more international schooling backgrounds. It is also important to note that none of these four students expressed a plan to study abroad. This position may be typical of the students who experience problems when they do later decide to study abroad, as they did not attempt to develop their academic literacy for such purposes.

Each student's background, course expectations, teacher impressions and specific writing task will be described followed by a closer examination of their writer identities and writing game strategies based on their language choices. Some consideration is given to research question 6, "Do students recognize critical argument and writer identity as important to their advanced writing education?" A further examination of the language will focus on the Appraisal theory aspects of ATTITUDE, ENGAGEMENT and GRADUATION (Martin, 2000; White, 2005) in order to discuss the students' variation of engagement with their expressions, and attempts to interpersonally position their proposals and propositions.

\subsubsection{Case 1: Aya}

As explained in chapter 5, Aya spent one year of high school in England. Although she went through the typical compulsory English classes starting from grade 7 and finishing in grade 12, Aya explained in her first interview that her mother was an English teacher at a private language school and for ten years she studied conversational English with her mother and teachers from the US and UK. In addition, her high school was well known for its English 
language education program. Her English classes there were taught in English, except for more difficult grammar explanation, and she had assistant teachers from Ireland and Australia.

While she learned some English writing in Japan, she was challenged to write a 3-page paper on Sherlock Holmes while in England. The only other complete essay challenge she faced was the entrance exam for Midori University, which she said she did not have enough time to write well. In her Composition 1 course she did not write many essays; instead she mostly completed grammar worksheets. Her teacher for that course was in his late 70 s and had been teaching in the department for several decades. Aya explained in her first interview that she was not a confident writer, giving poor spelling, grammar and vocabulary as examples.

Aya's expectations of the course were fairly straightforward-she hoped to learn how to write a "proper essay". She explained this clearly in her first interview:

Jim: $\quad$ So in your Composition 2 course, what do you want to do or hope to do?

Aya: $\quad$ Well, with this program, I think I'll be able to write a proper essay. I think he's very good and he talks about the shapes of paragraph and essay - shapes of essays - so I think I can be able to write essays properly. (May 2)

Based on the introduction to the course given by her teacher Mr. Doi, Aya believed his discussion of the "shape" of paragraphs and essays was the right idea and that the course would meet her expectations. As for her teacher's expectations, in her first interview Aya expressed her concerns about meeting them. Having read the course information handout, she discussed concerns:

Jim: $\quad$ So looking at the plan, it looks appropriate?

Aya: $\quad$ It's very detailed... It's kind of difficult for me.

Jim: What's the difficult part?

Aya: It's - I can't really give the answer he wants us to have. (May 2) 
She went on to explain that she felt she had the creativity required to complete the tasks, but not the advanced writing ability.

Mr. Doi had initially started teaching the course in Japanese, but on the request of students, he switched to English and maintained teaching in English throughout the year, using only occasional Japanese. In the first observed class (conducted in Japanese), Mr. Doi explained academic writing features including thesis statements, connected points, persuasiveness, personal opinion versus interpretation, and subjective versus objective positions. These were all features of writing Mr. Doi expected the students to learn how to apply in the course tasks.

While she was initially very positive about Mr. Doi as a teacher, later in the year she expressed concern in two areas: 1) his emphasis on peer feedback and 2) his background in a field that was not relevant to her research topic. The two points were related, as Aya wanted less emphasis on peer feedback and more comments from Mr. Doi, but knew that Mr. Doi's area of expertise could not help her specifically with her topic.

Aya's writing task selected for the analysis was the research paper that was the main task of the second semester. Aya chose to write on the Shabina Begum case in the UK in which the Muslim high school student went to court to defend her right to wear a veil, or jilbab (a long gown that covers one's head). This presented several problems for Aya. Although she had the experience of being a student in a UK high school for one year, she knew very little about the Islamic faith, and did not actually know nor had ever knowingly come into contact with a Muslim person. She said that she chose this topic for her research paper because she wanted to write about a current event in which a high school student's own culture was misunderstood and mistreated by the policy-makers of the school.

\subsubsection{Aya as a writer and analysis of selves}

Based on this information, it seems that Aya had something to say about the general topic, and had some experience with it (to an extent), so if she had been able to write an authorial or autobiographical paper, she may have had fewer problems. However, she was encouraged by her teacher to write objectively and to attribute their ideas to outside sources or other authorities. Aya's attempts to write using a discoursal self made it very 
difficult for her to develop her thesis, as the rhetorical mode she felt most strongly connected to on the topic was a sympathetic one, rather than a one generated from her sources. She also implied an ethical rhetorical mode through a mention that religious practices "should not be insulted." She ultimately settled on an authorial self, with some elements of a discoursal self in her attributions, as she felt it was most persuasive for what she was trying to argue. For example, she referred to the situation in Italy and cited exPrime Minister Tony Blair as a basis for making some claims:

Italian Government are undergoing the process of making a new law to ban wearing veil that covers their faces hoping that it will prevent terrorism to take place. As in Britain, former Prime Minister, Tony Blaire [sic] called veiling as "mark of separation". (Alam 30 2006) Considering these facts of conflict and as Europe being a non-Muslim society, it seems that minority culture is going through a tough time and the issue of veil is still heatedly discussed among the society but no solution is seems to be found.

As discussed in chapter 6, Aya struggled with the issue of ownership of her writing. Much of this was due to her peer group's disagreement with her thesis, and Aya's inability to convince them. She felt she identified personally with her thesis, wanting to present the issue of Shabina Begum's case from a non-Western perspective-precisely the problem Miss Begum faced as a Muslim in England. However, her peers were asking Aya to take a Western perspective, which Aya felt was "unfair" and caused her to feel disconnected from her paper (Aya, November 22).

The writing game strategies Aya used most were what Casanave (2002) named "interaction with texts and with others about texts," especially incorporating ideas discussed in interaction with others, about her text, and to a lesser extent, "blending voices." This second game strategy of Aya's attempt to blend voices, explained in section 8.2.2, resulted in a convoluted development of Aya's thesis. In her attempt to blend her voice with those of her peers', her voice became lost, as she did not agree with her peers, but attempted to compromise her own position in order to include theirs.

Even though she explained in peer discussions that she believed Shabina Begum should have been allowed to wear the jilbab in her school, Aya never actually stated this in her 
paper. Attempting to incorporate feedback from her peers, she took a fairly weak stance on the topic attempting to reveal something about the issue through an analysis of the Shabina Begum case. This was in line with her explanation that she tended to be neutral in her writing, as discussed in section 7.2.1. She offered some interpretation as well as some evaluation of her source evidence. Since there was no clear intention with her thesis other than that she would "analyze" the case, the evaluation of the evidence was not overt. Aya presented a number of quotes from a number of Newsweek and BBC reports and the Muslim Council of Britain for information on the court case. Aya's position on the topic was that neither the school nor Miss Begum's lawyer were arguing the issue from the same perspective as Miss Begum. After first presenting some quotes from Newsweek and BBC, she wrote:

It seems to be appropriate that the school was fighting on the ground of school uniform, its characteristic and fairness of when making it. It is also to the point that Yvonne Spencer [Miss Begum's lawyer] was fighting back on the ground of education to win the case However, Shabina Begum was not on the same ground as the school was. She was not even on the same field as her representative.

Aya then went on to provide a number of quotes from Miss Begum's interview with Newsweek in order to generate sympathy about her obligation to follow the rules of her religion in wearing the jilbab outside her home. Aya supported this with a quote from the interviewer who similarly wore a hijab both as an act of defiance and as an act of "defining [her] identity." Finally, in an attempt to blend her voice with those of the authors, Aya introduced a debate about the "obligation" of wearing a veil, pointing out that some interpret that it is the right of each individual to decide to wear a veil, while others understand that the Muslim society of which the individual is a member makes such decisions.

Aya extended the argument with supporting evidence about globalized Muslims and how traditions such as wearing a veil become more significant outside Muslim countries. She then started the second-to-last paragraph with an antithetical statement about how Muslims need to "be aware that their community co-exists with [the British]," and finished 
with a statement on the need for Britons to understand the importance for Muslim females of wearing a veil. In the end, she left the reader wondering what to do about the culture gap she described, writing, "There seem to be no effective solutions yet."

With such little background in and knowledge of policymaking and even less so of the Islamic faith, it was difficult for Aya to put herself in the position of writing a persuasive research paper. Her attempts at persuading her readers of her thesis that Miss Begum should have been allowed to wear her jilbab in school, which she never actually stated, were mostly superficial attempts at evoking an emotional response (not typical of the objective, discoursal self the teacher was expecting). Ultimately, Aya convinced neither her class of her thesis, nor her teacher that she had done work beyond a $\mathrm{C}$ grade.

In terms of Aya's recognition of argument and identity as important to her writing education, her choice of topic very much brought these issues to the front. Her own identity and writing on the issue of a student's need to establish her identity came together as the most important aspect in developing her argument. She felt she sacrificed certain aspects of her own identity in an attempt to meet the expectations of her peers and teacher.

Regarding the points raised in section 2.2.1 in relation to deductive and inductive writing, shared cultural knowledge between readers and writers, and mimicking features from sources, Aya experienced some difficulties. By not providing a definitive thesis statement in her introduction, this suggested Aya was not using the preferred deductive style. The cultural knowledge of her topic was not shared with her peer readers, causing serious problems for her decisions about her claim, leaving her to take a neutral stance. Finally, in terms of mimicking features of her sources, Aya had trouble integrating quotes and blending her voice with her sources, indicating that she did not mimic features, and instead used, however awkwardly, her own voice.

\subsubsection{Aya's language use}

The following table shows the number of phrases Aya used that matched the preset indicators within the Appraisal framework. The complete analysis of Aya's essay can be found in Appendix D. 


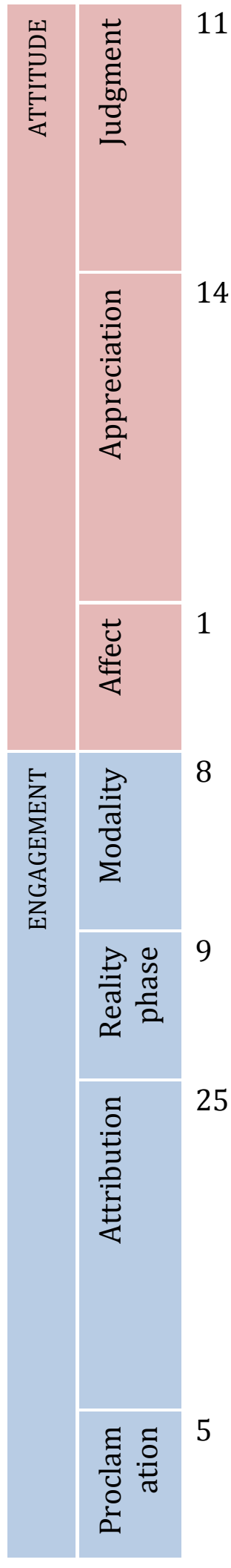

11 It is still rather unusual event to see women; One hardly has chance to see women in a veil; the school took it for granted; it is understandable that Yvonne Spencer to emphasized Shabina Begum's right; It seems to be appropriate that the school was fighting on the ground of school uniform; It is also to the point that Yvonne Spencer was fighting back; Shabina Begum was not on the same ground as the school was; She was not even on the same field as her representative; it is quite obvious that she has very strong feeling; community is indirectly expecting and welcoming women to wear veil; the interpretation of faith and its practice of her should not be insulted

14 issue of whether to let Muslim women to wear veil in pubic or not has been casing huge discussion; minority culture is going through a tough time; The point Shabina Begum intended to make and what was important for her was different; Her claim... is more to do with the religion; Her claim was not just about school uniform versus jilbab; Shabina Begum's court case may sound extraordinary; Qur'an... and other reliable sources; Islam is becoming more influencing; Islam greatly influences Muslim's daily life and decision-making; It is deeper than discussion of school uniform; It is strongly related to creation of her identity and her life itself; There seem to be no effective solutions; gaps between two cultures caused by misinterpretation of heart of the issue; it is not straightforward problem that can be solved in one flick

1 This paper is... eager to find out what causes huge discussions

8 It should be reminded; it can be considered that the school took it for granted; Shabina Begum's court case may sound extraordinary; the interpretation of faith and its practice of her should not be insulted; Muslim side should be aware; it needs to understand; it may causes more complicated discussions; They both need to find the way to respect each other's cultures

the situation seems to be very different; it seems that minority culture is going through a tough time; no solution is seems to be found; it gives an impression that there is certain gap; it seems that the point; It seems to be appropriate; There seem to be no effective solutions; religion seems to be at the core; it can be considered that;

25 In 2003... Jacques Chirac; He claimed that; Dalil Boubakeur shows concern; According to BBC News, Rome; As in Britain... Tony Blaire; According to the article, "Seeing Clearly"; Court of Appeal ruled in favor of Shabina Begum, saying that; Miss Begum called the ruling; she also stated that; the school claimed; They also said; from BBC NEWS online; Yvonne Spencer, according to BBC NEWS online... said; By reading these statements; As she states that; She says in her article, "Beyond The Veil"; As reading these sentence; According to interview with professor lyama; Fareena Alam says in her article; According to her saying; He points out that; They said that; They also state that; the head of MCB, Iqubal Sacranie made declaration as following; as Fareena Alam described young British Muslims

there is no chance for Shabina Begum to get justice; it can be said; there is no absolute reason; In fact, the Council considered; It is time for both Muslim society and British society to stop seeing the issue only through their perspectives 


\begin{tabular}{|c|c|c|c|}
\hline & 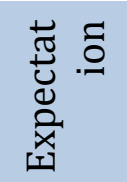 & 2 & $\begin{array}{l}\text { It will take a huge amount of time; there is a chance that the changes are going to be } \\
\text { made }\end{array}$ \\
\hline & \multicolumn{3}{|c|}{ No Counter-expectation } \\
\hline \multirow[t]{2}{*}{ 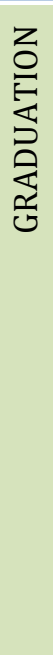 } & 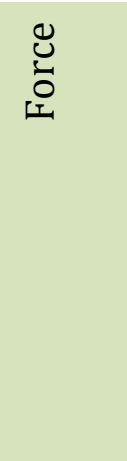 & 23 & $\begin{array}{l}\text { It is still rather unusual event; even in a huge city like Tokyo; One hardly has chance; } \\
\text { has been casing huge discussion; what causes huge discussions; the act of wearing veil } \\
\text { had not always been seen; something that the students must wear in proper way } \\
\text { without an exception; they had given enough consideration; just because she did not } \\
\text { wear school uniform; She was not even on the same field; statements which were } \\
\text { strongly connected; it is quite obvious; she has very strong feeling; wearing jilbab plays } \\
\text { vital role in her daily life; discussed only by the field; there is no absolute reason; there } \\
\text { are several numbers of them; Islam greatly influences Muslim's daily life; religious } \\
\text { belief plays vital role in creation of her identity; seeing the issue only on the ground; It } \\
\text { is strongly related to creation of her identity; seeing the issue only through their } \\
\text { perspectives; a huge amount of time }\end{array}$ \\
\hline & 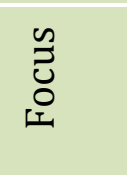 & 7 & $\begin{array}{l}\text { the issue of veil is still heatedly discussed; The judgment did not affect her directly; she } \\
\text { did not mention about her right to have education particularly; strictly speaking; what } \\
\text { is a heretic thought or true belief; Muslim community is indirectly expecting; purely out } \\
\text { of her will to please God }\end{array}$ \\
\hline
\end{tabular}

ATTITUDE. Aya used a high number of expressions of Judgment (11) and Appreciation (14), indicative of a sympathetic rhetorical approach. However, she used only one Affect phrase-interestingly, this was in her thesis statement. This seems to reflect the struggle Aya experienced with not wanting to take a side, preferring to maintain neutrality. What she wrote, This paper is...eager to find out what causes huge discussion... expressed the desire to explore the topic, rather than speak authoritatively on it. Aya explained in her follow-up interview that her thesis had been shaped by the work she had done with her peers, who disagreed with her pro-jilbab-wearing leanings, attempting to present their ideas in her paper. This is the particular indication of her writing game strategy of interacting with others.

ENGAGEMENT. The very high number of expressions of Attribution (25) shows that Aya attempted to blend her voice with others, positioning herself using at times Modal expressions (8), Reality phases (9), Proclamations (5), or Expectations (2). She used the voices of her authors to further emphasize the sympathetic rhetoric of her argument. The problem with her attempts to use this game strategy was in her awkward attributions. 
Although she made attempts at introducing her sources providing author information when available, the attributions of her ideas to these sources disrupted the coherence of the paper. For example:

According to BBC News, Rome, last updated $7^{\text {th }}$ November 2006 by Christian Fraser...

According to the article, "Seeing Clearly" written by Carla Power and Rebecca Hall, in Newsweek, 27 November 2006 edition...

As reading news materials and protests that had been made by the high school from $B B C$ News online, it can be considered that...

Aya was required to use a minimum of five sources for her assignment, and she did practices in class and received feedback from Mr. Doi on how to cite them in her paper, but the final version showed a lack of ability to effectively blend her voice with those of the authors.

GRADUATION. The language Aya used to value interpersonal impact was fairly consistent throughout the paper. She used both expressions of Force (23) and Focus (7). Most of the Force expressions were used to raise interpersonal impact, including even, not even, just, only, strongly, just because, etc. The Focus expressions were fewer in number and were used to sharpen the semantic value of the phrase. Aya's voice was clear in the GRADUATION language she used, but it was not used in relation to sources, therefore she was not blending her voice with theirs.

\subsubsection{Case 2: Ai}

In her initial interview, Ai explained that she had taught herself, using a popular self-study book recommended by a teacher, to write essays for her entrance exam to Midori, having only done basic grammar practices in school. She had the traditional experience of six years of English classes in junior and senior high school in Tokyo public schools that Ai thought may have had some emphasis on English education, but she was unsure. At that time she wanted to improve her academic writing as she had hoped to study abroad. However, she changed her mind about studying abroad after enrolling at Midori, deciding it might pose a problem with her job-hunting plans. Ai was particularly grateful to her Composition 1 
teacher the year before, continually referring in her interviews throughout the year to the wealth of knowledge she gained in that course.

At the start of the course Ai was excited about the opportunity to write research papers, as she wrote one in Composition 1 that she believed was the most significant task in developing her writing skills. Ai expected that Ms. Ellis would teach her how to find appropriate sources, as this was a particular problem for her in the previous year. She also hoped Ms. Ellis would help her with her conclusions, a self-professed problem area in her writing. Ai was confident early on that the course would meet her expectations.

In the beginning of the year, Ai understood Ms. Ellis to be a good, enthusiastic teacher with a well-planned course and who provided extensive, useful feedback, similar to her Composition 1 teacher. Later, she expressed some frustration with the amount of time Ms. Ellis would spend on tasks other than writing essays, including summaries of sources, thesis statement writing, and learning to cite both English and Japanese sources. In her June interview, she expressed disappointment:

Ai: I thought we would write more essays, so now I am not satisfied with the class.

Jim: So far in class, what writing have you done?

Ai: $\quad$ What writing? I would say just, thesis statements, or supporting sentences. And different topics. And with, we memorized what is for example, what is the counter-argument or hook or refutation. And we did a test of that. But I think memorizing these, a word a day, is not useful. It's useful but, yeah. We cannot write a good essay only with this knowledge. We have to practice writing. (Ai, June 21)

In her November interview she expressed concern about spending an entire class on one handout practicing citations. She also expressed frustration about the long delays in getting teacher feedback, sometimes several weeks after submitting a draft. When asked about her progress, she was unsure:

Jim: So in terms of your own development, of your writing skills, how is it going? Since April?

Ai: $\quad$ Since April? I'm not sure because there are not many feedback. I need more quickly. Quick feedback. So... but I heard that from one of my friends, she said 
in [teacher]'s class, Eigo Kenkyuu [English Research], I think, [teacher] said to her, your writing skills are going down. So she was shocked.

Jim: Do you think that may be happening to you?

Ai: I'm not sure because recently I don't write essays. So... I'm not sure. But I'm sure... (November 15)

Ai chose for her research paper topic, schools in Japan for the deaf. This was the paper chosen for the text analysis. Ai chose to write on this topic as she was concurrently taking a course on American Sign Language (ASL) at Midori and was enjoying it. In that course she read Deaf News every week and came to the conclusion that Japanese deaf people lacked the confidence of deaf people in Europe. She believed the answer was in generating awareness by teaching sign language in schools, and increasing deaf students' chances of mainstreaming into public schools. Outside of her exposure to the topic in her ASL course, the only other experience with deaf people was watching a school friend communicate with her deaf mother. Ai believed this sparked some of her curiosity.

\subsubsection{Ai as a writer and analysis of selves}

Ai found it strange and frustrating how she was taught in university to write on topics that interested her, but that she had to remove personal opinion. In her December interview, Ai said:

I thought it was strange to remove personal opinion because for example, in the entrance exam, I wrote personal opinion or experiences, and my high school teacher said it was ok, but once I entered the university, I never use, be able to use, so I felt strange that... if I could write personal opinion in my research paper or argumentative essay, maybe it's more, it's getting more interesting I think, for the readers. (Ai, December 14 )

The issue was that Ms. Ellis, like Ai's Composition 1 teacher the year before, insisted the students write objectively, essentially requiring students to write with a discoursal self by attributing all the ideas in their papers to outside sources. Although Ai understood the requirement, she struggled to fulfill it. Ai's sense of writer identity, not unlike Aya's, emerged in her research paper with two mentions about "identity," in this case, the identities of deaf people in Japan. Sign language, Ai explained in her paper, gave deaf people a language (if it were not for sign language, the deaf would not have any language), and with 
that language, they would form their identities. Ai's use of ATTITUDE phrases indicated more of an authorial self, rather than discoursal, but she did manage to avoid emotional phrases. Ultimately both discoursal and authorial selves were present in the text as many of the ideas were attributed to her sources, but many were stated as objective truths. Her authorial self was largely a product of her own personal beliefs. In terms of research question 6, this strongly indicates that Ai found the issue of identity to be an important consideration in her argument.

The main writing game strategies Ai used were "speaking with authority," and to a lesser extent, "interacting with texts and with others about texts." Her authority came through in the high number of ENGAGEMENT (36) phrases, sometimes made as part of the interpretation of her supporting sources. The ATTITUDE phrases Ai used were generally value judgments, putting herself in the heroic role of defending the rights of deaf people to give them more "chances" and "opportunities." Her point was that this would be done through raising the awareness of hearing people by teaching them sign language, and by increasing the number of schools for the deaf. She wrote:

Thus, more schools where deaf students can learn in sign language should be founded in Japan because...it would enable them to enter the academic world, offer them an opportunity to feel proud of being deaf and treated more equally in education.

Nowhere in the paper did she offer evidence of deaf students being mistreated or treated unequally, nor did she provide any evidence that deaf people are not part of an "academic world."

While the number of Attribution phrases was also high (12), the interaction was more with others about her text, rather than with the texts Ai used as supporting sources. Her use of her sources was generally to provide limited facts. She used the same attributive phrases repeatedly, for example in the article or in the book, and presented the information for the purposes of then making interpretations: this means, from this research it can be said that... Her interaction with others about her text occurred in in-class peer sessions. As discussed in section 6.2.1, Ai was very positive about the peer sessions, wanting to adapt her writing style to take on some of the features of her peers' writing. 
Regarding the points raised in section 2.2.1 in relation to deductive and inductive writing, shared cultural knowledge between readers and writers, and mimicking features from sources, Ai experienced some difficulties, but fewer than Aya. Ai provided a relatively clear thesis statement in her introduction, but it did not address the whole argument, indicating she was reserving the greater claim for the conclusion and suggesting that she was not using the preferred deductive style. It was not clear whether the cultural knowledge of her topic was shared with her readers, but there was a sense of assumed shared knowledge, as she did not provide definitions of key terms. She also opened the essay with the rhetorical question, "In which language do deaf students study better, sign language or Japanese?" assuming her readers would agree that this was a comparable choice. Finally, in terms of mimicking features of her sources, Ai was more successful integrating quotes and blending her voice with her sources, indicating that she may have mimicked features of the sources.

\subsubsection{Ai's language use}

Like Aya, the number of phrases Ai used that matched the preset indicators within the Appraisal framework were similarly Judgment-heavy. The complete analysis of Ai's essay can be found in Appendix D.

Table 11: Summary of Ai's language use

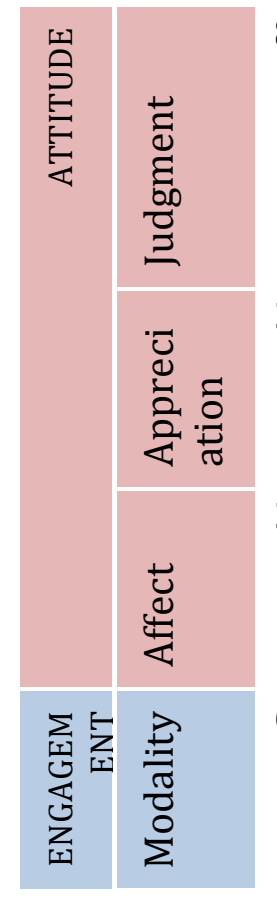

8 This means that they feel less reluctance; can express more details and richer content; learn higher-level content in sign language; Deaf children can learn about what the deaf community is; acquisition of sign language is considerably important for deaf persons; deaf students can have more chances to secure an occupation; the experiences of learning sign language itself and other subjects in sign language are significant; they could catch up to regular students

3 sign language is indispensable as a deaf person's mother tongue; two kinds of sign language in Japan makes the problem even more difficult; for deaf children, who do not understand Japanese grammar, the latter is difficult to comprehend

3 deaf students can feel proud of being deaf; Deaf students become able to communicate with others without feeling fear; they would have more of a reason to feel proud

6 more schools where deaf students can learn in sign language should be founded; deaf students should learn in sign language; Japan should promote more familiarity; those who want to teach using their ability of signing could can make the situation better; some people might say that; Japan should have more schools for deaf students 


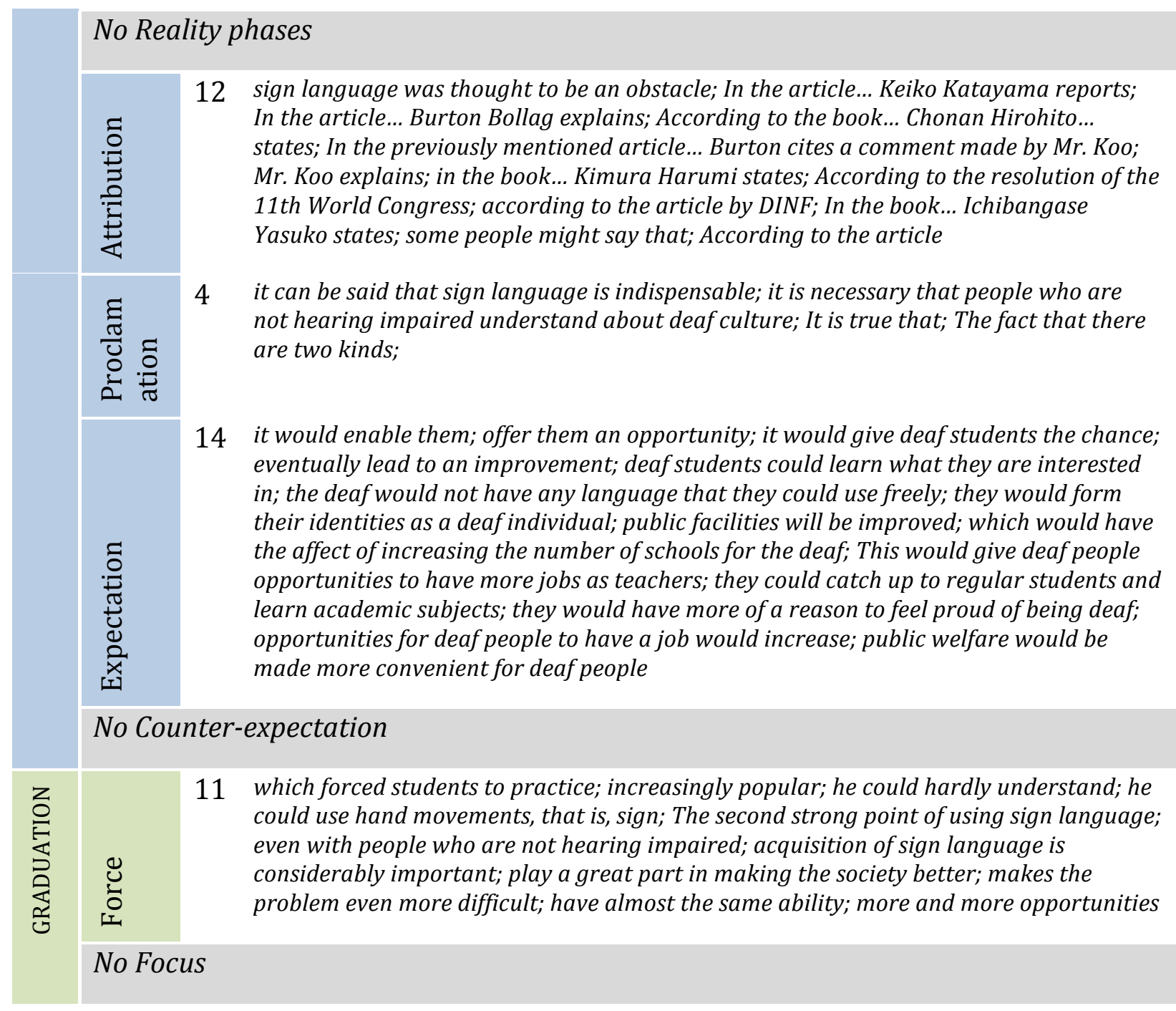

ATTITUDE. Ai's use of Judgment (8) and Appreciation (3) phrases, like Aya, indicates a sympathetic rhetorical approach. Although Ai did use some Affect (3) phrases, they were used mostly as value judgments, avoiding personal emotional response perhaps in an effort to write objectively. Ultimately her argument was sympathetic and judgmental, describing deaf people's assumed difficulties and frustration and that by learning sign language, the deaf could catch up to regular students and learn academic subjects. ATTITUDE phrases made up the bulk of the argument of Ai's paper, indicating Ai had resorted to an authorial self. The issue for Ai may have come down to her choice of topic. She chose a topic on which her stance was based on the assumption that deaf people were somehow victimized by Japanese society, without any evidence, other than news that the first school for the 
hearing impaired in Japan was opening soon. From a discoursal representation, the discussion would have been very one-sided, but with an authorial self, Ai was able to make strong statements based on assumed shared knowledge with her readers.

ENGAGEMENT. Ai took an awkward stance in her thesis statement, giving four reasons to insist that the number of schools for the deaf in Japan needed to increase, citing a news report that the first accredited school for hearing-impaired students was to open in Tokyo in the following year. With no Reality phases, and proportionally only some Proclamations (4) and Modality (6), Ai still maintained a certain sense of authority over her claims throughout the paper. She used more Attribution (12) for both hearsay and a number of sources. The sources were attributed consistently to provide a basis to make her claims, using the same attributive expressions repeatedly, but she did not attempt to interact with the texts through challenging the authors. The issue was that her paper presented no counter-argument-no suggestion that Japan should not found more schools for the hearing impaired, so her insistence of increasing the number of schools ended more awkwardly than her thesis. She finished her essay with a sentence fragment:

To increase the number of schools for deaf students from now on, more and more opportunities for people to know about the deaf community and the importance of sign language for deaf people.

The high number of Expectation phrases (14) indicates a reliance on shared assumptions with her reader, an observation further supported by her use of Force phrases.

GRADUATION. Ai's interpersonal impact was rather minimal with no Focus phrases, even though there were a high number of Force (11) phrases. The Force phrases (the second strong point; is considerably important; even more difficult, etc.) were used as part of the assumption of shared knowledge with her readers that deaf people were victims of an unaware Japanese society, and were not related to her supporting sources, further establishing her use of the writing game strategy of speaking with authority over interacting with the texts. 


\subsubsection{Case 3: Rika}

Although Rika's international background was quite different to Ai's more traditional background, the results for their final papers were similar. This was probably due to the fact that they had the same teacher, even though Rika was in Composition 1 while Ai was in Composition 2.

Rika's background, discussed in chapter 5, made her comfortable with using English. In her first interview, Rika said she loved English. She was open to whatever her teachers wanted to teach her, but had no specific plans to use English in the future. Rika was a confident writer in English having spent five years from the age of seven in public schools in England, and then from the age of twelve, attending schools in Japan that had specialized English language programs. However, she credited all of her English ability with the time she spent in England. The teachers of English in her first school in Japan were Japanese, and taught English in Japanese. In high school she had native English teachers and wrote several essays.

In her first interview, Rika felt Ms. Ellis's course was appropriate, she liked Ms. Ellis, and she looked forward to the course as it was described in the course outline. Rika explained that the hardest part of writing for her was "thinking... organizing ideas is difficult, critical is difficult... Difficult for me for how to start the essay" (April 27), but she was unsure if Ms. Ellis would be working on these skills. Even at the end of the first semester, Rika commented, "I'm not good at thinking" (June 22).

In her second interview (June 1), Rika felt she was "kind of" able to work on the skills she wanted to, as she had already had lessons on how to write an essay, including practices with thesis statements and hooks as well as using supporting evidence for all the different types of essays she would be writing in the year. She liked the materials that included model essays with comprehension questions, and understood that practicing the features of the essays before actually writing the essays was important. She had not learned how to write thesis statements before and was still unsure about using them to express an opinion. In her third interview, toward the end of the first semester (June 22), Rika felt the course was meeting her expectations, despite the three class cancellations due to Ms. Ellis's poor 
health and despite the fact that there had been very little actual writing practice, still focusing on essay features rather than writing essays. She continued to struggle with starting the essay with a good hook, but felt that writing a thesis statement was becoming much easier.

In the second semester, Rika said, "It's getting difficult" (November 15). They were starting to write essays, and Rika struggled with putting together the features of the hook, thesis statement and supporting evidence she had practiced in the first semester. In her December interview, she explained she was struggling with the summary homework as she had trouble choosing what information to use. Rika explained that usually when she is given an essay topic, she just "write[s] about what [she] know[s]" using personal experience. When I asked her if that meant her essays tended to be personal, she replied, "Most of them are, but some of them are kind of like, knowledge." In choosing the information to use in the "knowledge" essays, Rika explained, "I mainly choose things that feels right for me... things I want to know about" (December 13).

In her January interview at the end of the year, Rika commented on the lack of actual essay writing (January 23). In her second of two follow-up interviews, she complained that the course was "kind of boring" (June 17, 2008). Although she found the practices on thesis statements in the first semester and citations in the second semester difficult, Rika felt Ms. Ellis spent too much time on these practices. Rika felt the sample essay used to practice doing citations was long and tedious, so having to use that essay for so many weeks was not enjoyable. Ultimately, however, Rika admitted she thought the skills, along with the practices involved in writing an objective argumentative essay, were important and helpful.

The writing task selected for the analysis was Rika's argumentative essay on animal testing for which the thesis was: Using animals for disease research should be stopped... She chose this topic from a list of suggested topics provided by Ms. Ellis. The students were required to write pro and con statements for each suggested topic before choosing one of them. Rika described the topic option of space exploration as particularly difficult, and home schooling as too unfamiliar. She also avoided the public smoking topic for fear it would have been too popular. Although she had no personal interest in the topic of animal testing, Rika felt it 
was the easiest of the topics to work with, as she had some level of familiarity about the topic having watched some TV shows on it and debated the topic in high school. She explained that the thesis and ideas in her essay came from that debate and from quick searches on six different Internet sites for common related issues. Rika admitted that she did not concern herself about the reliability of the websites, as that aspect was not required for the task. The task simply required a minimum of three sources.

\subsubsection{Rika as a writer and analysis of selves}

Rika was very receptive to the challenge of writing an objective, argumentative paper using outside sources as many of the skills required such as a particular type of thesis statement and citations were new skills. She commented on her satisfaction with learning how to make her writing less personal and avoid using the pronoun 'I'. For Rika, the Composition 1 course showed her for the first time the possibility of showing a discoursal self in her writing by attributing ideas to outside sources, and showing an authorial self by taking up a strong, authorial stance and stating her personal ideas as subjective truths. In her December interview she acknowledged, "I sometimes make my personal experience like a general thing" (December 13). She successfully combined the two selves for her argumentative paper, although the discoursal self was minimized by more attributive phrases of hearsay than clearly attributing the sources.

Writer identity was not discussed directly, but Rika's discussion of impersonal writing revealed that she did not necessarily feel as connected to her essay without using the pronoun ' $I$ '.

Jim: Do you still feel like, when you write like that, is it still you? Is it still your ideas?

Rika: Not really...it's difficult not using 'I'. Very difficult.

Jim: Do you think, if the essay's built on what you want to know, does that make the essay more personal?

Rika: I don't think so. Maybe it is but...

Jim: ...the notes from [teacher] say "Don't be subjective." But do you think that there should be some subjectivity in your essays? Or should they just be completely objective? 
Rika: I think most of them should be objective, but I think it is better to have some own opinions. (December 13)

From this discussion it is apparent that Rika feels that objective writing means impersonal writing, and that means removing herself and her opinions from the writing, which she finds difficult and not as good as writing that includes her opinions. Thus, the resulting combination of authorial-discoursal self was a way for Rika to feel satisfied with her attempt to meet the teacher's expectations using impersonal language, but still asserting her own opinion and taking a strong, authorial stance.

The main writing game strategy Rika used was "speaking with authority." Because of the peer exercises and use of teacher feedback on drafts, she was also able to use the strategy of "interacting with texts and with others about texts," though there was little of this apparent in the actual language use in her essay. It is interesting to note that these were the same two strategies used by Ai, also a student of Ms. Ellis. Rika's authority came through in the very high number of Judgment (9) and Appreciation phrases (17), many of them in statements of objective truths and shared assumptions with the reader. She made several sweeping generalizations including:

This is because people only think about their own profits and do not think about animals' rights.

If people knew how their tax money was being used for animal testing... most people would condemn the use of their tax money for animal testing.

Rika's sense of authority also came through in some value judgments, including:

Improvement of technology allows alternatives to be used instead of animals, so animals should be freed and people should think about how to live in the world peacefully together with animals.

It is not just humans who are living in the world.

White (2005, p.6) explains that such value judgments are the result of writers expressing personal "rules and regulations" of social expectations and value systems, essentially 
asserting their own moral code. In terms of interacting with texts, Rika did directly attribute two individuals in Japan, one woman a member of the Japan Anti-Vivisection Association (JAVA), and one man a professor at Midori, clearly localizing the argument. She took a quote from the JAVA member and used it to support her own statements about people's ignorance about animal testing. The quote from the Midori professor was actually part of a section of the paper where Rika showed an element indicative of the Japanese writing organization pattern, ki-shou-ten-ketsu (discussed in section 2.2.1) through use of a topic shift in the development of her second of three supporting ideas. On the issue of spending tax money on animal testing, Rika suggested:

Instead, tax money currently used for animals testing should be used for environmental conservation and pollution preservation.

The quote from the university professor was on the most important problem in the next century being global warming. She used it to support the topic shift, which she then developed into an issue of living standards. She brought the topic back to animal testing with the final sentence in the paragraph:

From this perspective, it is much more efficient to use money for improving people's standard of living rather than kill innocent animals in vain.

Regarding the points raised in section 2.2.1 in relation to deductive and inductive writing, shared cultural knowledge between readers and writers, and mimicking features from sources, Rika experienced fewer difficulties than Ai or Aya. Rika provided a very clear thesis statement in her introduction, addressing the whole argument, indicating she used the preferred deductive style. It was not clear whether the cultural knowledge of her topic was shared with her readers, but as she did provide definitions of terms, this suggested that Rika was aware of a potential lack of readers' knowledge of her topic. Finally, in terms of mimicking features of her sources, Rika was less successful integrating quotes and blending her voice with her sources, using very little paraphrasing. This indicated that she did not mimic features of her sources. 


\subsubsection{Rika's language use}

\section{As Rika's paper was not meant to be a research paper, the number of phrases Rika used}

\section{that matched the preset indicators within the Appraisal framework was somewhat lower}

than the two Composition 2 students, particularly the Attribution phrases. The complete analysis of Rika's essay can be found in Appendix D.

Table 12: Summary of Rika's language use

\begin{tabular}{|c|c|c|c|}
\hline \multirow[t]{3}{*}{ 䆓 } & 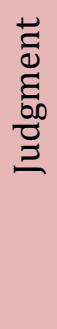 & 9 & $\begin{array}{l}\text { because people only think about their own profits; it is inhumane to use animals just } \\
\text { for the profit of humans; If people knew how their tax money was being used for } \\
\text { animal testing despite no reliable results from research, most people would condemn } \\
\text { the use of their tax money for animal testing; it is inhumane to use animals just for the } \\
\text { profit of humans; many people are only thinking about themselves; many people are } \\
\text { becoming aware; It is not just humans who are living in the world; It is time for } \\
\text { humans to take action and fight for peace, both for humans themselves and for } \\
\text { animals }\end{array}$ \\
\hline & 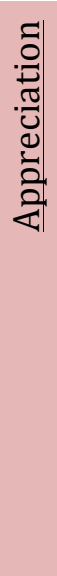 & 17 & $\begin{array}{l}\text { it is not the most reliable method; too much tax money is used for the experiments; } \\
\text { animal testing is not the most reliable method; This means it is very dangerous to use } \\
\text { animal testing; incorrect data from animal testing leads to incorrect knowledge for } \\
\text { treatment; there will be a risk of more people suffering from side effects; too much tax } \\
\text { money is used for animal experiments; Animal testing costs a lot of money; This means } \\
\text { that environmental problems are becoming more and more serious; unreliable data; } \\
\text { they cannot speak and are weaker than humans; Animals have the right to live too, } \\
\text { and animal testing is an infringement on their rights; there are many negative points } \\
\text { about animal testing; There are alternatives to animal testings today, which do not } \\
\text { cost as much as animal testing and are effective and humane; Improvement of } \\
\text { technology allows alternatives to be used instead of animals; improvement of } \\
\text { technology enabled companies to test medicines without animal testing and use } \\
\text { alternatives such as human skins instead; the amount of tax money used for the } \\
\text { experiment is too much }\end{array}$ \\
\hline & 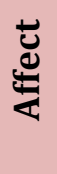 & 3 & $\begin{array}{l}\text { Not being able to speak, however, does not mean they do not have feelings; Although } \\
\text { animals cannot speak human language, it is not difficult to imagine that they will } \\
\text { suffer when medicine does not work; Animals born for the sole purpose of research } \\
\text { could not possibly imagine that they were born to be killed }\end{array}$ \\
\hline \multirow[t]{2}{*}{ 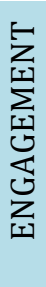 } & 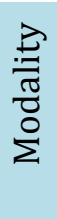 & 8 & $\begin{array}{l}\text { Using animals for disease research should be stopped; it does not always mean it will } \\
\text { be safe; serious side effects may show on humans; tax money currently used for } \\
\text { animals testing should be used for; animals should be freed; people should think about } \\
\text { how to live in the world peacefully together with animals; it is more likely to say; if } \\
\text { people can dismiss their preconceptions about medicine }\end{array}$ \\
\hline & \multicolumn{3}{|c|}{ No Reality phases } \\
\hline
\end{tabular}




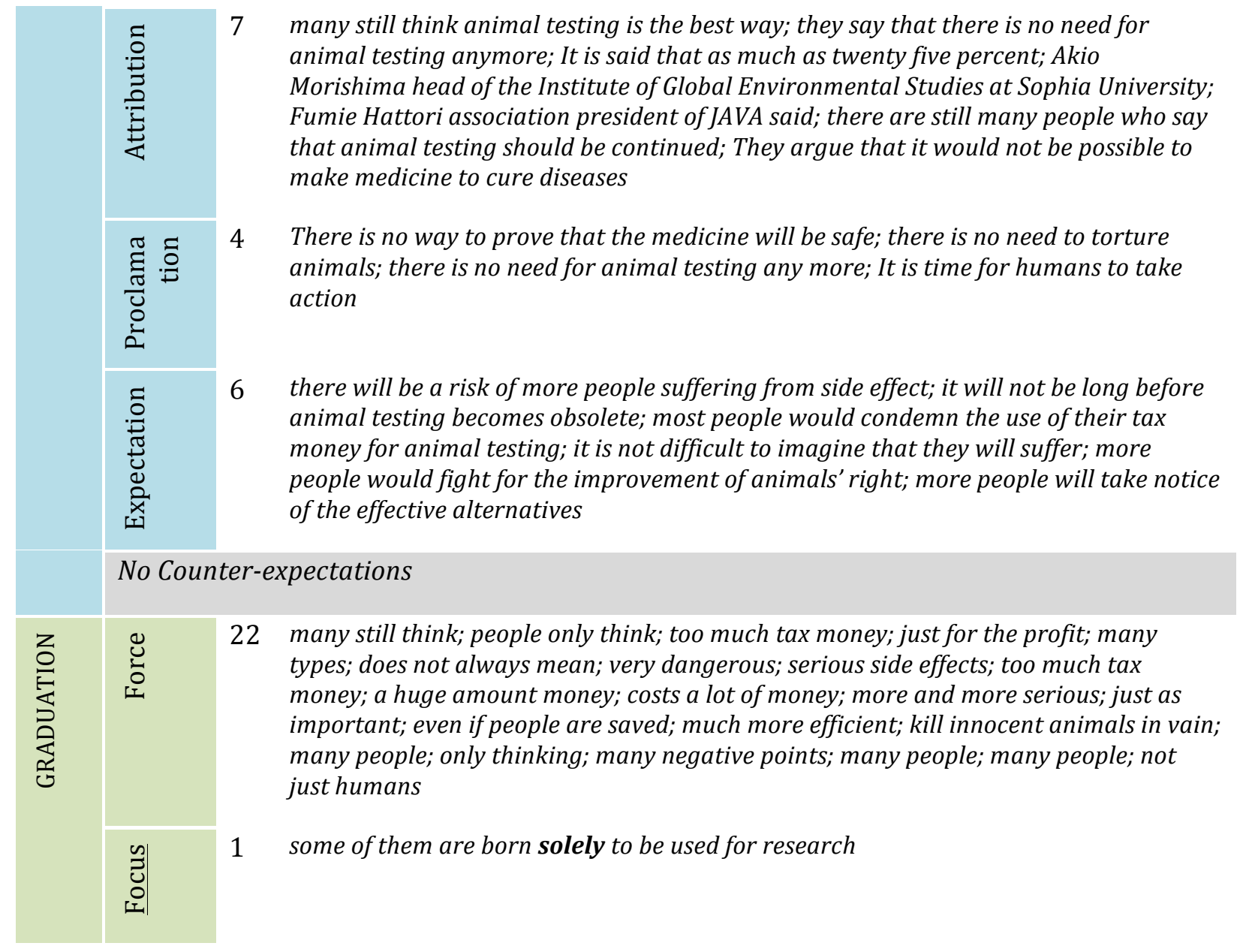

ATTITUDE. Phrases of ATTITUDE were used more than any other in Rika's essay, with a very high number of Judgment (9) and Appreciation phrases (17) and some Affect phrases (3). Much of the judgment was made on the topic itself and high level of assumption on shared knowledge with her reader, as there were a number of generalizations about people's ignorance about animal testing. She also made a number of value judgments, calling animal testing inhumane and describing animals as forced to be ill. This added to Rika's assertion of her own social expectations and moral code. More judgments were made on animals' rights, describing animal testing as an infringement, and blaming people for not thinking about their rights. The ATTITUDE was strong and authoritative, indicative of a strong, authorial stance.

ENGAGEMENT. Although Rika used a number of ENGAGEMENT phrases, it was evident in the analysis of this indicator that Rika may have struggled with her connection with the paper, 
as she had described in a follow-up interview the feeling that by removing personal language from the essay, she felt the ideas were no longer hers. For example, after providing ideas attributed to outside sources, Rika on two occasions followed source information with This means... in order to try to show ownership of the idea. She also on two other occasions followed source information with generalizations, for example:

Fumie Hattori, association president of JAVA said, "People don't know about the issues... When they do learn what's going on there's often a good response," (Bayer). Still many people are only thinking about themselves...

However, the Modality (8), Proclamation (4), and Expectation phrases (6) all suggest Rika did manage to stay somewhat connected to her paper. As for the number of Attribution phrases (7), this would have indicated some attempt at displaying a discoursal self, but the phrases were mostly hearsay, further displaying the authorial self.

GRADUATION. With the high number of Force phrases (22) and one Focus phrase, Rika maintained a particular interpersonal impact in her essay that was based on urgency, using the phrases very dangerous; too much tax money; costs a lot of money; just for the profit. These phrases showed Rika intended to persuade the reader of her thesis through awareness raising of the extreme problem of animal testing.

\subsubsection{Case 4: Yui}

Yui was the one student in the group who felt more than any other that the level of English her teacher Mr. Clark aimed at was too low. As explained in chapter 5, Yui spent seven years in the US, until the age of 14 when she returned to Japan and enrolled in an international school taking most of her courses in English. Although she had written many papers in English, some several thousand-word essays and a two-year long research paper in high school, she still lacked confidence, particularly with essay organization and structure.

At the beginning of the year, although she explained that she liked Mr. Clark, Yui was reluctant to make an assessment of the course he had prepared. In her first interview, she commented on the course outline: 
It's not too detailed. So I'm - but - ok. I know like after a few weeks go about like what [teacher]'s gonna - how he's gonna teach and then maybe I'll feel something if he's teaching what I want. (April 26)

By her second interview, Yui was growing frustrated with the worksheets they were using in class on comma placement and writing titles, saying:

I want to write something - it's a writing class. And I'm sure everybody's thinking that too because we did this in class and he gives us homework too. I'm not sure I'm learning anything in that class... So I hope to learn something by the end of the year. (May 31)

In general, the course was moving too slowly for Yui:

I hear from like other people in the writing class - they think it's really way too slow and we're just doing something too basic. (May 31)

In terms of Mr. Clark's plan to work at the pace of the students, he explained in his May interview:

I can go with the pace of the students. I'll say, "Okay, this is, kind of, what I want to teach you. If it's difficult, okay, we - let's take our time. There's no hurry. I wanna make sure you understand this." If, oh, yeah, they know topic sentences and supporting sentences and concluding sentences and they understand parallel structure, okay, "Wow, you guys have just gone through all of this. Well guess what? Now we have time to do what you wanna do." (Mr. Clark, May 10)

Yui was very frustrated with this approach:

It's just that it's not like we completely don't understand it, we just wanna see if we're right or not. It's not like we don't understand, but we just you know - if we had a mistake, we can correct it... It's just that it's not something we need to go over in class - so I don't really say anything in class. (May 31)

She did eventually become concerned she was being too critical of the course, saying "I don't wanna say bad stuff about that class, but I just wanna feel more doing something... I wanted to say that he should expect more." (May 31). Yui went on in that interview to describe the jealousy she felt of friends' experiences of not being able to finish the homework for their Composition 1 classes because they were writing so much. By her June interview, Yui was relieved they were writing, but explained that she did not appreciate the topic of video games, as she was not interested in it. She explained that the task was short, so she was unsure if the peer reading exercise was worthwhile: "it was 150 words - so I 
don't know if it was worth reading or not" (June 21). That interview ended with continued frustration:

But there's a lot of returnees in that class and people around me are all returnees and when they went to high school or middle school they said they wrote more than this. They know how to write. (June 21)

In the beginning of the second semester, Yui seemed to take a slightly different perspective, saying:

Yui: Overall impression - I would say it was understandable - not hard it was easy - I was able to understand what he was saying - the whole thing. And I think he could have moved on a little bit faster, but we basically did the basic things of writing.

Jim: Do you feel generally positive about the course last semester?

Yui: Positive. I didn't - we didn't write as much. We just talked about how to write and how sentences - what comes after topics and a main-idea sentence and kind of talked about it and did some writing.

Jim: Ok - so you're satisfied with that?

Yui: I wanted to write a little more than just paragraphs. The whole spring semester was about writing paragraph - just one paragraph - about a topic... It was how he explained what sentence needs to come at the beginning of a paragraph and how we can conclude it. And the structure. I think it was a nice review. (October 10)

But Yui came back to the point that her friends in other classes were writing more, saying:

What he really cares is if I have the topic sentence - everything that he wants us - he checks every single thing - is the word capitalized - is it in the middle. I won't really want him to care about those, but what's inside like how you put stuff inside... I've seen my friends from different writing classes write a lot of stuff and they first write what they think is right and the teacher's feedback is, "You should write the real name here so it would make more of an impact and this one needs to come over here so it would make more sense." So I want - if you write more and get those feedbacks you would learn more, right? Next time you'd be like oh this would make more sense if I put the sentence over here. (October 10)

She finished the October interview with continued concern:

Jim: Do you feel as though this class is helping you to improve your writing?

Yui: Not really. I guess I'm just comparing it with my other writing classes. But maybe it's helping me because I know the order of writing now. But I don't 
know if it's improving my writing skills. I know how to organize stuff but I don't know if I can write good sentences or good strong statements and stuff.

Jim: Do you have a chance to write essays in any of your other English classes?

Yui: No, not at all. (October 10)

In her end-of-year interview, Yui's appreciation for the course stayed fairly basic, and isolated to that course, saying:

I think - I still have to learn a lot more - that's what I'm thinking right now - about writing. But in order to do well in that class that [teacher] taught, I think I can do well in that class, but for like writing - if you take writing as like outside of that class - I still have a lot to learn. And I still don't know how to write what I really want to write. Express my feelings in writing and stuff, which I really wanna do. So I'm thinking I need to learn more. (January 22, 2008)

The tasks Yui was given in the first semester included many worksheets on formatting, capitalization and punctuation as well as practices on the writing process including brainstorming, outlining and drafting, followed by a few paragraph-writing tasks. In the second semester Yui wrote six short essays of approximately 300-400 words each with multiple drafts. Each essay was designed to focus on a different essay type, including process, division-classification, compare-contrast, cause-effect, problem-solution, and argumentative. The students were given a choice of topics from a list of usually three or four suggestions.

The paper selected for the text analysis was her essay on global warming. Yui explained in her November interview that she chose the topic because, "I want to write something that I really need to think about" (November 13). It was a struggle for Yui, explaining that the brainstorming process was good for turning random ideas into one main idea she could use, but explained, "I really don't use it [brainstorming] for writing an essay" (December 11). The essay, Yui explained, was written more to fit the specific guidelines set by Mr. Clark than to express her own ideas. 


\subsubsection{Yui as a writer and analysis of selves}

Yui really struggled in her Composition 1 course to develop her writing skills in a way that she felt was worthwhile. Repeatedly in interviews she mentioned the point that she wanted to write more in order to organize her own ideas in a way that was effective and more clearly expressed her ideas. Mr. Clark's very specific expectations did not allow Yui the freedom she felt she needed to write in a way that allowed her to develop the way she wanted to as a writer. Mr. Clark emphasized the importance of students brainstorming their ideas and using their own ideas to write their essays, but the writing tasks as they were designed were not well received by Yui. She felt restricted, even though she felt she understood why Mr. Clark designed the tasks the way he did. She understood there was no need to write objectively, as she had been told repeatedly in her writing classes before coming to Midori, but did not have a clear grasp on writing from experience without writing a narrative, or some kind of autobiographical paper. Mr. Clark was clearly directing students to write authoritatively, which Yui did reluctantly.

In terms of the issues of writer identity and critical argument, Yui did not discuss these points specifically, but did return often to the idea that she wanted to write about her own ideas. In her December interview I asked her about not representing herself in her writing:

Jim: In terms of wanting to know how to represent yourself in your writing what you've been working on so far in [teacher]'s classes is to not represent yourself?

Yui: Right - sort of.

Jim: But it's still your opinion, right?

Yui: Yeah, but you can't use the word 'I' and if you can't use the word 'I' it's not your own experience it's what the majority of people think about that topic... What I wanna write is - these essays have like "I lost the chance" and everything is like 'I.' It's like no one else knows how you felt at that time. And like what I did in his class - I think everybody has the same - if you give the topic, everyone will have the same kind of essay. So you might have some ideas and stuff, so I think everybody can do that, but - I think it's a different type of essay. (December 11)

The issue of ownership of the writing was an important one to Yui. As discussed in section 8.2.6, Yui felt the essays she wrote were not really her own, that the style of the essay was 
"already made" (Yui, November 13). The result was a combination of autobiographical and authorial selves, apparent in the content of the high number of ATTITUDE phrases (see next section), using content based on her own life experiences, seemingly precisely what $\mathrm{Mr}$. Clark was requiring.

The writing game strategies Yui used were "making the paper look right," and to a lesser extent, "speaking with authority." Mr. Clark's specific task parameters stuck to genre conventions and rhetorical structure. He taught students to write essays using fill-in-theblank outlines from which students were expected to copy their thesis statement (what Mr. Clark called "main idea sentence") and topic sentences into their essay. The genre conventions were specific to the point of requiring the thesis statement start with the word although, discussed in section 8.2.6. Yui was required to write five-paragraph essays of approximately 300-400 words in length, without exception. Therefore, as these restrictions did not allow for much paragraph development, the papers she wrote were about making the paper look the way Mr. Clark required. In terms of the writing game strategy of "speaking with authority," Yui's supporting ideas all came from the brainstorming step in the writing process, and not from outside research. She presented the information using many Appreciation, Modality, and Force phrases, suggesting a voice of authority, though this was limited as it was not in relation to any other voices, as there were no outside sources used in Yui's essays.

Regarding the points raised in section 2.2.1 in relation to deductive and inductive writing, shared cultural knowledge between readers and writers, and mimicking features from sources, Yui experienced the least difficulty. Yui provided a clear thesis statement in her introduction, indicating she used the preferred deductive style. It was clear that Yui assumed the cultural knowledge of her topic was shared with her readers. The support for her thesis was locally contextualized, providing familiar situations that her readers living in Japan could relate to. Finally, in terms of mimicking features of her sources, as Yui did not use any sources, this was not a consideration. 


\subsubsection{Yui's language use}

Because Yui's paper was only approximately 400 words and she used no outside sources, the number of phrases Yui used that matched the preset indicators within the Appraisal framework was significantly lower than those of Aya, Ai and Rika. The complete analysis of Yui's paper can be found in Appendix D.

\section{Table 13: Summary of Yui's language use}

\begin{tabular}{|c|c|c|c|}
\hline \multirow[t]{3}{*}{ 突 } & Judgment & 12 & $\begin{array}{l}\text { If people consider overpackaging, First, always remember to turn off the } \\
\text { lights when leaving the room. Second, do not let the water run while } \\
\text { brushing your teeth or washing your face. Third, do not set the air } \\
\text { conditioner to an excessive temperature. Just always keep in mind the word } \\
\text { "mottainai" and you'll be somewhat contributing towards saving energy. Just } \\
\text { say you do not need a plastic bag when going grocery shopping and say you } \\
\text { do not need chopsticks when buying food at the convenience store. Carry } \\
\text { your own shopping bag and your own chopsticks. There is no need to wrap } \\
\text { every candy, then wrap it again with a bigger bag, then put in a bag, then } \\
\text { wrap the box, then put it in a box again. There is no need to use cars to go } \\
\text { somewhere nearby. Walk to the nearest station and take buses and trains. } \\
\text { Also, do not use one car per person. We should start carpooling with friends }\end{array}$ \\
\hline & Appreciation & 6 & $\begin{array}{l}\text { irregular weather is causing people to suffer; will change the world; } \\
\text { companies making candies and snacks should consider overpackaging; Once } \\
\text { is enough for wrapping; it will lead to us having less trash; This means fewer } \\
\text { vehicles, which means less carbon dioxide in the air }\end{array}$ \\
\hline & No Affect & & \\
\hline \multirow{6}{*}{ 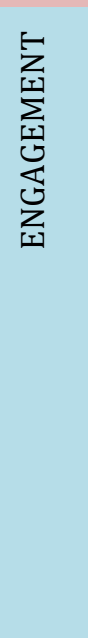 } & Modality & 7 & $\begin{array}{l}\text { we should stop wasting energy; have minimal trash; we should rethink about } \\
\text { our way of transporting; We should start carpooling; have to be done by } \\
\text { many people; We should all reconsider about what we have to do; which is } \\
\text { necessary to save our own lives }\end{array}$ \\
\hline & Reality phase & 2 & The world seems like it's coming to an end; seems like a little thing \\
\hline & No Attribution & & \\
\hline & Proclamation & 2 & $\begin{array}{l}\text { There is no need to wrap every candy; There is no need to use cars to go } \\
\text { somewhere nearby; }\end{array}$ \\
\hline & No Expectation & & \\
\hline & No Counter-expe & ctati & \\
\hline 怘 & Force & 12 & $\begin{array}{l}\text { many people; every degree, every minute lights are turned off, every } \\
\text { millimeter of water saved; many people; Just always keep in mind; somewhat } \\
\text { contributing; just say you do not need a plastic bag; every candy; then wrap it } \\
\text { again with a bigger bag, then put in a bag, then wrap the box, then put it in a } \\
\text { box again; many solutions; many people; not just one person; all reconsider }\end{array}$ \\
\hline
\end{tabular}


ATTITUDE. The high number of Judgment and Appreciation phrases (18) in Yui's very short essay presented an authorial voice of urgency on the issue of global warming. Since there was no requirement to use outside sources, Yui did not use any. Casanave's (2002) point that undergraduate student writers learn to mimic the authorial voice they become familiar with in university does not apply to Yui as there was no authorial voice from other sources for her to mimic. Instead she used her twelve Judgment and six Appreciation phrases as "cries for help," perhaps reflecting her own feelings of struggling to come to terms with Mr. Clark's specific writing instructions.

ENGAGEMENT. As Yui's essay was approximately 400 words, there was little room for expressions of ENGAGEMENT. Also, as there were no outside sources, there were no Attribution phrases. Yui seemed to place a great deal of emphasis on obligation with seven

phrases showing modality of obligation, but these were nearly all the same value, mostly using the word should. With Yui's only two Reality phases (using the word seems) and two Proclamations (using the phrase there is no need) in addition to the repetitive modality, this showed a very low level of interpersonal communication. She admitted in interviews she felt disconnected from the essay.

GRADUATION. Yui used a high number of Force phrases (12) and no Focus phrases. The many Force phrases, unlike the modality of obligation, were more varied. This helped to make the low interpersonal impact of Yui's essay somewhat less apparent.

\subsubsection{Summary}

The four students focused on in this section showed a variety of experiences and struggles with their task of writing a persuasive, argumentative paper. Their teachers prepared the students for these tasks using textbooks, handouts, and their own ideas about what students needed. Casanave (2002) raises the issue of whether teachers should be teaching students to conform to formal writing conventions or to resist them. This point in particular reveals the disparity between the teachers' approaches on these otherwise 
similar tasks. Mr. Doi emphasized the importance of peer feedback, encouraging students to focus on communicating their ideas to the satisfaction of their peers. Ms. Ellis spent a great amount of time on practices with thesis statements, citations and other skills students needed to use for their papers. Mr. Clark presented students with very specific writing conventions to which students were required to conform.

The students responded in very different ways. Aya struggled with the peer work process, disagreeing with the "Western" perspective of her peers and wanting to assert a distinctly non-Western thesis, one she felt more strongly connected with. In the end, Aya chose to write to the expectation of her peers, causing her to take a very neutral position, and feel disconnected from her paper. Ai struggled with her teacher's approaches, often complaining in interviews of the amount of time spent on skills, and not enough time on writing. Rika had similar complaints about the same teacher, but ultimately felt positive about the skills she had learned. Yui felt underwhelmed by her teacher's low-level approaches, and felt the writing skills required to work with ideas were never developed.

Dual possibilities for selfhood were apparent in each student's paper. The common combination was an authorial-discoursal self, where students attributed ideas to sources, but also stated their own ideas as objective truths. Only Yui's writer identity was different, displaying an authorial-autobiographical self where all the ideas were related to her own life experiences, and for which she took responsibility of the authorship, although reluctantly.

The writing game strategies used varied according to both the students' intentions with their writing, and with their teacher's expectations-particularly on the point of writing objectively. While three of the students in these case studies used the game strategy "interacting with texts and with others about texts," it was really only Aya who played this as her main strategy, attempting to work with her peers' comments, teacher's feedback, and to use another game strategy of "blending voices" to combine her voice with those of her outside sources. Both of Ms. Ellis's students, Ai and Rika, used the same combination of strategies, focusing mostly on "speaking with authority" as their way of being personally involved in a paper that was required to be objective. Yui was the one student who most 
clearly used a particular game strategy, "making the paper look right." This was a clear requirement set by her teacher, so Yui made sure she wrote the paper to his expectations.

As for language use, all four students used more Judgment phrases than any other type, with Attribution phrases coming a close second. The use of these phrases strongly supports the dual authorial-discoursal self. In the case where Yui did not use sources, the ideas used were solely from her own personal experience, displaying a dual authorialautobiographical self.

\subsection{Chapter summary}

The writer identities and writing game strategies used by the student participants in this study revealed the level of negotiation necessary to complete the writing tasks in these classes. The students attempted to be persuasive in their writing by using any or all of the different possible selves, sometimes in contrast to their teacher's occasionally explicit, occasionally vague instructions. Students attempted to meet their teacher's expectations of an objective, discoursal representation of themselves in their writing, but since they did not have the expertise required by many of their topics, they resorted to a more authorial voice. As confirmed by the students in their interviews, the students in the $\mathrm{C}$ class who used an autobiographical self in addition to an authorial self did so with a certain level of confidence that was not apparent in some of the other students' writing. This suggests that students are ill prepared to write with a discoursal voice. The analysis of the students' texts using an Appraisal Theory framework revealed that the students used a great deal of ATTITUDE, indicative of an authorial self, in their attempt to persuade their readers, often with very subjective language.

The students employed the writing game strategies unconsciously and, depending on which game strategies were used, the strategies required them to think critically about their writing. The students' development of critical thinking skills appeared to hinge on whether or not they needed to be analytical in their writing, and how much freedom of choice they were given with the task. Students who felt constrained by strict instructions from their teacher (which was the case for most students, but especially those in the A, B and C classes), or who received contrary feedback from peers (such as Aya in the D class), 
seemed to find the critical thinking aspect of their writing task less important in completing the task.

In the next chapter the six main research questions will be answered in depth, using examples from the analysis of the literature and research data. 


\section{Chapter 9. Writing and reasoning revisited: Responding to the research questions}

This chapter provides in-depth responses to the six research questions of the study. Having reviewed relevant literature and established the methodological framework, data were analyzed in order to answer the six research questions. The responses to each research question take into consideration issues raised by the literature as well as the analysis of the data. In this chapter, numbers in parentheses (e.g. 1.3) in sentences and following citations indicate the section in the thesis where the issue was discussed.

\subsection{Research questions and responses}

This section responds to the six research questions raised at the end of chapter 3 , generated before data were collected. After collecting the data, it was discovered through emerging theory that the focus of these questions on developing critical argument and establishing writer identity was appropriate. In chapters 6 and 7, it was revealed that the common practices between courses were on writing as a communicative act (acknowledging the reader, chapter 6) and writing as an exercise in critical thinking (developing the thesis, chapter 7). The questions were:

1. What is the current practice of English writing education in relation to critical argument and writer identity in a Japanese university?

2. What are teachers' goals for these writing courses?

3. What are teachers' identifiable cultural expectations of EFL writing?

4. What practices do teachers bring to the classroom to develop critical argument and writer identity in students' writing?

5. How effective are these practices in terms of students' writing output?

6. Do students recognize critical argument and writer identity as important to their advanced writing education?

It is important to note the difference between research questions 1 and 4. Question 1 refers to the "current practices" in terms of teachers' philosophies on the role of critical thinking in university EFL writing, while question 4 refers to actual "classroom practices" in the form of writing activities. For research questions, 2, 3 and 4, Ivanič's (2004) discourses of 
writing framework, used at first minimally in the analysis of the observation data, proved to be particularly useful in framing the answers to these questions, focusing on classroom goals, expectations, and practices. Finally, the original intention for answering these questions involved more of the students' perspectives of their teachers. However, in their interviews the student participants proved to be less aware of and/or concerned with their teachers' expectations and goals. Therefore, student interview data are used only for questions 3,5 and 6.

\subsubsection{Current practices}

The first research question was: What is the current practice of English writing education in relation to critical argument and writer identity in a Japanese university? The instruments used in collecting the data included classroom observations and interviews with the teachers. The focus in the observations was on teachers' efforts to encourage students to think about critical argumentation through critical thinking activities.

As discussed in chapter 2, the central issue regarding current practices is that although Japanese higher education policymakers have decided to encourage the development of critical thinking skills, it is a continually neglected area. Academics and researchers have pointed to several reasons and justifications for this. I highlighted the arguments, such as those related to Japanese collectivist thinking, that attempt to explain why Japanese students are seen to lack critical thinking skills (2.2.2). However, academics such as Stapleton (2001) found that his students were able to write with "individualized voices, which [were] closely related to critical thinking ability" (p. 534).

With these ideas in mind, I approached the classroom observations with an eye on critical, communicative writing practices. As discussed in chapter 6, all courses required students to write for a particular reader or readers, not usually the teacher, but often peers, or imaginary readers. Students were also made aware of the communicative nature of their writing tasks as part of a negotiation with readers in the writing process. This negotiation was discussed as part of the power dimension of developing critical literacy and as a way to bring writing matters into sharper focus for students and teachers (Canagarajah, 2002) (2.1.5). 
Critical thinking as part of writing was observed in two forms. One was the focus on critical thinking in practice tasks with developing a thesis, discussed in chapter 7. Students were observed spending a great deal of time on brainstorming their ideas and discussing appropriate language for expressing them, and ultimately establishing a position or claim. They used, in varying degrees, personal experience in these practices. The other form of focus on critical thinking observed was the students' acceptance and/or refusal of the classroom materials or practices.

The teachers discussed their own ideas about current practices in university EFL writing and how that affected their approaches to the writing assignments they gave. All four teachers agreed that ideas about current practices in university EFL writing focused on practical skills and writing as a communicative social act (Rinnert \& Kobayashi, 2001) (2.1.3). These ideas were, however, interpreted differently by each teacher.

Mr. Doi explained in his interview that he gained much of his understanding of what needed to happen in university EFL writing classes through his own experiences as an ESL writer in his postgraduate studies in North America (5.4.1.3). He felt it was very important for students to be analytical in their writing in order to develop critical thinking skills. Mr. Doi was observed in an early class saying, "Please analyze, rather than describe. In order to analyze something you sometimes need to be angry" (Mr. Doi, D class, May 30) (7.2.2). In terms of the "anger" Mr. Doi spoke of in class, he went on to explain to the students that anger allows writers to be "emotionally engaged" so a "strong reaction" would provide the necessary energy to be analytical. This approach seems to fit into the descriptions of the principles of Japanese writing, particularly haragei, translated as "force of personality" by Davies and Ikeno (2002) (2.2.1). Without the emotional engagement, the force of personality (or writer identity) may not be apparent. The other principle discussed in section 2.2.1 was aimai (ambiguity), which Mr. Doi also discussed in his class (as part of the discussion on stereotypes, 7.2.1), but as a feature of writing unique to Japanese, and not English writing.

Also providing a native-Japanese perspective, Ms. Aiba explained that her understanding of current university EFL writing practices came from her experiences teaching and 
researching language education in North America. In her interviews, Ms. Aiba was acutely aware of the lack of writing tasks set by other lecturers in the department, and therefore chose to go against prescribed ideas about academic essay writing practices for exam purposes (discussed in section 2.3.3 as a problem of EFL education in Japan according to Poole 2005). She explained that she did not want students to write essays in whatever way they understood they should write them (many of the students who spent 5-7 years abroad were in her classes), only to then have to instruct the students to "unlearn" previous strategies that may have been appropriate for their contexts at the time. Instead she chose to focus the students on advanced grammar usage first, and later on, a variety of genres that they might actually come across in the near future, rather than on essay writing skills that they may never use. These genres required students to use a genre-appropriate voice or writer identity, but when we discussed this point in interviews, Ms. Aiba rejected the idea of teaching concepts of writer identity in class. Her intention was strictly an attempt to bring authentic writing tasks into the classroom. Students were observed expressing frustration, discontent and a lack of understanding about the purposes of such practices in their writing class (7.4.2).

Mr. Clark and Ms. Ellis, the two native-English teachers, had very different understandings of current practices in university EFL writing.

Mr. Clark's understanding was based on his many years of experience teaching EFL writing in Japan. Over the years, he had come to focus on the basic strategies for composing an academic essay. These basic strategies took into account the Ministry of Education's emphasis on more communicative writing, as observed by the amount of emphasis Mr. Clark put on identifying a reader for all the students' writing. Mr. Clark was aware of this emphasis as he was actively conducting his own research in EFL education. However, the strategies did not take into account the emphasis on critical thinking, as he felt students needed to focus on the mechanics of writing, rather than concern themselves with thought processes. Mr. Clark's assignments had very rigid requirements, including fill-in-the-blank outlines, so there was little room for negotiation in the writing tasks. In an interview, Mr. Clark explained that students needed these rigid parameters in order to avoid confusion 
and especially ambiguity (2.2.1), one of the strongly maintained principles that influence Japanese writers (Atkinson, 2002; Casanave, 2002).

This last point was also an issue for Ms. Ellis in her Composition 1 class where the topics were assigned, but not an issue in her Composition 2 class where the students chose their own topics. Ms. Ellis's understanding of the current practices in university EFL writing was fairly limited. She relied heavily on textbooks to supply "rules" for academic writing. In observations, the students appeared to understand the textbooks and the assignments, and spent a great deal of time discussing the effectiveness of language use for clarifying thesis statements (7.2.1) and persuading a reader (6.4). In her interview, Ms. Ellis explained that she had no guidance for her courses, having only been provided with course titles. There were no expectations of the courses whatsoever. Therefore, as a conscientious new teacher, she chose reliable textbooks as the basis for her courses. The textbooks did not, in fact, emphasize communicative or critical aspects of writing, but Ms. Ellis felt these points were crucial based on her own experience as an academic writer.

To sum up, the current practices in university EFL writing in Japan are meant to reflect increased emphasis on communicative writing and developing critical thinking skills. The teacher participants in this study were all observed practicing elements of communicative writing and critical thinking skills development, but in their interviews, not all teachers accepted this idea. Mr. Doi placed a great deal of stress on writing communicatively and critically through emotional engagement and force of personality, thus encouraging his students to develop critical thought while establishing writer identity. Ms. Aiba's tasks required different writer identities for different genres, but she rejected the idea that there was a focus on writer identity and stressed the importance of authentic texts in a more practical approach to writing skills development. Ms. Ellis also stressed communicative, critical writing in her Composition 2 class, encouraging students to establish a clear position as the goal in the writing process. Finally, in the Composition 1 classes, Ms. Ellis limited the communicative aspect of writing, focusing more on critical thought in developing a thesis while trying to utilize elements of Japanese essay writing, and Mr. Clark took a formulaic approach that concentrated more on format than on communication or critical literacy. 


\subsubsection{Teachers' goals}

The second research question was: What are teachers' goals for their writing courses? The instruments used in collecting data to answer this question included the teachers' course syllabuses and interviews with the teachers. In their interviews, the teacher participants discussed their philosophies behind their goals for their writing course(s). These philosophies were based on previous teaching experience (Ms. Aiba, Mr. Clark), or on suggestions made by colleagues whom the teachers felt were more expert writing teachers (Mr. Doi, Ms. Ellis). In addition, when designing their courses all four teacher participants took into consideration the proficiency level of the students.

The goals of the teachers varied, as reflected by the various discourses of writing applied in their classes (5.4.1). Critical argument (or critical thinking) and writer identity were discussed in interviews. When asked about writer identity, Mr. Doi admitted he had not thought about it, and the other three teachers rejected establishing writer identity as a goal even though it was evident that it played a role to a certain extent in all the teachers' courses. As for developing critical argument, Mr. Doi and Ms. Ellis felt it was an important goal, while Ms. Aiba and Mr. Clark explained that structures and forms were more important (to be discussed in the following section). Of the five criteria that frame Ivaničs (2004, p.225) discourses, the two I use to answer the question of teachers' goals are beliefs about writing and beliefs about learning to write.

In terms of her beliefs about writing, Ms. Aiba's social practice discourse, unique in the study, was based on the idea that writing should be purposeful and applicable to a social context. Her beliefs about learning to write were therefore based on the idea that writing in real-life contexts was the best method of teaching and learning. These beliefs shaped the goals of her course and the writing tasks in the second semester. However, this was a shift from the first semester skills discourse approach when students were focused on grammar. Ms. Aiba's beliefs for the first semester were that students needed the grammar base in order to use the language effectively and appropriately according to the genres of the writing tasks in the second semester. These beliefs (2.3.3) are supported by Yoshimura (2001) and Matsuda (2001) who explain that Japanese students do better with forms of 
writing as it gives them a starting point for the otherwise unfamiliar possibilities of English writing strategies.

Mr. Doi's beliefs about writing were of a sociopolitical nature, in which writing goes through a negotiated and contestable process in a social context. His beliefs about learning to write were based on his own experience as a second language academic writer of English. Mr. Doi concentrated on the directness of English in contrast to the vagueness of Japanese (2.2.2 and 6.2.2). The goals of the course were focused on understanding why different types of writing differ, and establishing a position among opposing, alternative positions in developing critical thinking skills. He therefore introduced a variety of text types in the first semester to encourage students to understand the differences between them. Then, in the second semester he required students to negotiate and contest their research papers in peer groups. Such practices are supported by Casanave (2002) and Stapleton (2002a) (2.2.2), who describe student writers' attempts at meeting the expectations of assessors. They explain that students need to develop their critical thinking skills and understand the strategies needed to meet those expectations.

Similarly, Ms. Ellis's E class (Composition 2) goals were also based on beliefs about writing of a sociopolitical nature, but with more emphasis on creativity and the writing process. In her F class (Composition 1), although most of the course goals focused on essay features and forms, four of them addressed arguing and thinking (5.4.2). These beliefs were also based on her own experience as a writer in her second language-Japanese. Writing in Japanese (2.2.2), focuses on jiyu (freedom) and jibun no iken (one's own opinion). Ms. Ellis explained in her interviews that learning to write academic Japanese taught her that thought processes should be revealed in academic writing and that this could only be achieved through a certain level of knowledge about the subject. This was why she encouraged her students to write on topics that interested them and that they wanted to know more about, as research would be required.

The teacher who stood in greatest contrast to the other teachers on beliefs about writing was Mr. Clark. Although there were similarities between his course and Ms. Ellis's Composition 1 course in that they both applied skills and process discourse approaches, 
Mr. Clark's goals for his students were of a strategic and formulaic nature. He focused heavily on writing rules, and did not encourage creativity beyond the use of the students' own personal experiences as supporting evidence for the topics. He explained in his interview that this was because, in his experience, the students needed the basics of academic language and essay structures more than developing critical thinking skills. This belief is supported by Phelan (2001) (3.5) who claims that "practical wisdom", in the form of classroom strategies, is much more pedagogically effective than overt teaching of critical thinking skills.

In summary Ivanič's (2004) Discourses of Writing Framework proved to be very useful in framing the answer to the second research question. The different identifiable discourses helped to establish a basis for understanding the teachers' goals in their courses, which were confirmed through interviews with teachers about their course syllabuses. Ultimately, the teacher participants had strikingly different goals. Ms. Aiba's goals were focused on different forms of writing, while Mr. Doi's goals were more about generating a wider sociocultural understanding of writing in EFL. Ms. Ellis's goals in her Composition 2 class were based on her understanding of writing in Japanese, allowing students a certain amount of creativity and freedom. Mr. Clark's goals were the most rigid, requiring students to meet very specific parameters of writing tasks. Ultimately students' awareness of critical thinking skills through their writing courses depended very much on their teacher's ideas about the courses.

\subsubsection{Teachers' cultural expectations}

The third research question was: What are teachers' identifiable cultural expectations of EFL writing? Data from interviews with both the teachers and students, and the students' written texts were used to answer this question. The particular focus of this question is on the students' writing and their understanding of their teachers' expectations, which were discussed in the students' interviews. The cultural expectations of the teachers were discussed in their interviews. Of the five criteria that frame Ivanič's (2004, p.225) discourses, the one that can be used to best answer this question is assessment. Those teachers using a skills discourse approach assessed for accuracy, and a genre discourse approach assessed for appropriacy. A creativity discourse approach meant the teacher 
looked for interesting content and style, mostly in the form of allowing students to choose their own topics. The social practices discourse approach meant assessing for effectiveness of purpose focusing on particular writing constructs, and a sociopolitical discourse approach meant assessing for social responsibility focusing on content.

Based on assessment criteria, the course goals were fairly categorical. In the Composition 2 classes, Mr. Doi's (D class) process, genre, creativity and sociopolitical discourses of writing approaches meant the assessment was focused on content, rather than language use. He looked more for interesting and meaningful content, but with a certain expectation of utilizing the features of the given genre of writing. Mr. Doi explained in his interviews that he felt Japanese students tend to have a fairly sufficient background in grammar instruction before studying at university. This idea is supported by a number of researchers and academics who claim the main pedagogical approach in Japan's English language education is grammar-translation (Aspinall, 2003; Moore \& Lamie, 1996; Poole, 2005; Twine, 1991; discussed in chapter 2). Therefore Mr. Doi wanted to concentrate on developing students' critical thinking skills in order to encourage them to be stronger writers, which was much of the reasoning behind requiring such extensive peer feedback on assignments. This aspect of Mr. Doi's expectations also reflects his cultural background as a native-Japanese teacher with extensive experience with producing postgraduate ESL academic writing. As a postgraduate student in North America, he was regularly advised by his professors to be emotionally engaged with the content of his writing (7.2.2).

Ms. Ellis's (E class) skills, process, genre and sociopolitical discourses similarly meant that she concentrated on the content of her students' writing, but with fairly strict focus on accuracy as well. The E class students were often surprised by how many comments were written on their papers, both on content and grammar. In her interviews, Ms. Ellis described the need for more deductive rather than inductive writing from the students, supporting the ideas raised by Kubota $(1997 ; 1998)(2.2 .1)$, who argued that writers who can organize their thoughts in their L1 should be able to write well in the L2 with increased explicitness. As for the extremely detailed comments on grammar and language use, Ms. Ellis explained that she expected students to learn the benefits and importance of revision, supported by Matsuda (1997) (2.3.3), as part of the writing process. Ultimately, as a native- 
English novice teacher with limited background knowledge of EFL education and no guidance from the department on conducting her courses, Ms. Ellis based her expectations on those prescribed by the textbooks she selected for her courses.

Ms. Aiba (A and B classes) concentrated on the effectiveness of the writing, based on the accuracy and appropriateness of language and structural choices through skills, genre and social practices discourse approaches. Ms. Aiba's expectations were revealed through discussions in the interviews rather than through an examination of her assessment on written tasks. This is because the comments and feedback on students' writing was minimal. Ms. Aiba explained that she kept the comments and feedback minimal in order to allow these students, who were understood to have higher levels of proficiency than most students in the department, to make their own decisions about what revisions needed to be made on later drafts. Students complained about the lack of revisions and feedback in her classes. This raises the issue of the lack of revisions and discussions of revisions that were part of the academic writing process observed in the other three teacher participants' classes. Matsuda (1997) explained that without the revision process, the students' intentions in their writing are not discussed, which does not allow for students' thought patterns to be revealed (2.3.3).

In the Composition 1 classes, Mr. Clark's (C class) and Ms. Ellis's (F class) skills and process discourses focused mainly on students' ability to use language accurately and to make their essay structures fit into prescribed, correct structures. There was very little focus on content, although both teachers did comment on essay organization as this affected the development of ideas. This is much like the entrance exam writing tasks described by Kajiki (1996) (2.3.2), which are concerned more with grammatical accuracy than content. Both Mr. Clark and Ms. Ellis explained in their interviews that this decision was based on the understanding that Japanese students tend to have a thorough knowledge of basic grammar rules and structures, and should therefore be assessed on that knowledge, rather than on content, as they have limited experience with the subject matter of the papers. This idea is supported by Stapleton (2001) (2.2.2), who emphasizes the point that students are thought to be unable to display critical thinking skills in papers where there is less familiarity with the subject matter. However, both Mr. Clark and Ms. Ellis required, 
commented and gave feedback on their students' ability to clarify and support a thesiselements of EFL academic writing both teachers felt were counter to Japanese writing forms (2.2.1 and 2.2.2).

A further consideration of the teachers' cultural expectations was noted in the students' acceptance or refusal of the teachers' materials and course content (7.4). In Mr. Doi's class, the students were encouraged to be "critical" and even to be "angry." Mr. Doi became concerned about his control over the class when the students actually followed his instructions and openly criticized the textbook he was using that involved a lesson on reader versus writer responsibility in English and Japanese writing. The students continued to respond critically to course content and materials throughout the year, which Mr. Doi directed into group peer work of each other's writing. In the three other Composition 2 courses, which were more skills and genre-focused, the students expressed their strong discontent with the course content and materials by complaining in their interviews. In the Composition 1 courses there was more polarity. As Ms. Ellis aimed to challenge her students with advanced academic materials, the students were open and accepting of the materials and their teacher's explanations for using them. Mr. Clark's intention to cover the basics was not a challenge for the students, who seemed to either appreciate the easier comprehension of such materials, or in Yui's case, simply gave in and accepted them as part of her teacher's specific approach.

The varying degrees of leeway offered by the teachers in terms of students' ability or desire to critique the materials is perhaps telling of the cultural expectations of the teachers. Mr. Doi's non-language education background, teaching English Composition for the first time, and English being his second language, may have all contributed to his openness to encouraging critical thinking skills through critique of the course. Mr. Doi admitted in interviews that he was learning as he went along, and felt honesty and openness in the class would be a valuable approach. Ms. Ellis decided on a more controlled approach even though her background was similar. This might have been because although she was open to the idea of learning from her students about teaching English Composition, she was in her third year of teaching the courses at Midori and had established clearer ideas of what to 
expect from her students. Ms. Ellis and Mr. Clark both maintained highly controlled classes, both indicating that they had clear ideas of knowing what to expect from their students.

To sum up, the cultural expectations in these writing classes varied depending on the teacher's background and personal philosophy about learning EFL writing. The answer to the third research question was meant to be based mostly on the feedback on the students' drafts. However, the amount of feedback varied greatly between teachers. Ms. Ellis gave the most extensive feedback with papers covered in comments, which students felt was too much to deal with. Mr. Doi gave feedback that added to the extensive peer feedback he required of the students. The student participants from his class had mixed feelings about this, with one student disappointed, wanting less feedback from her peers and more from her teacher. Ms. Aiba's minimal feedback was intended to allow students to make their own decisions about revisions, but all four student participants from her two classes complained about the lack of feedback, showing a severe discrepancy between the teacher's and the students' expectations. Finally, Mr. Clark's feedback on papers focused mostly on formatting and language use, but did also draw students' attention to the thesis, which was meant to fit a specific formula, and to the development of the thesis with supporting ideas. Students responded positively to this feedback as they felt it was good that he did not correct their language use, but instead made suggestions for language clarity. As for the formulaic thesis statements, the students expressed satisfaction with this as they felt they understood Mr. Clark's expectation clearly.

\subsubsection{Developing critical argument and writer identity}

The fourth research question was: What practices do teachers bring to the classroom to develop critical argument and writer identity in students' writing? The instruments used in collecting data to answer this question included classroom observations and interviews with the teachers. As discussed in chapter 6, the teachers used various approaches in their classes. According to Ivanič's (2004) Discourses of Writing framework, there are at least six different approaches teachers may take in designing a writing course. Each approach suggests certain ways of developing students' writing skills. 
The sociopolitical discourse most acutely stressed developing the skill of critical argument, observed in the D and E classes where the creativity discourse was also observed in the teachers' decision to allow students to choose their own argumentative writing topics. Particularly in the D class, in learning critical argument the students also developed a sense of their writer identity. Mr. Doi's emphasis on reader versus writer responsibility in comparing Japanese with English writing (7.2.1) led to discussions of cultural stereotypes where identity, his and the students', became a focus.

Mr. Doi's decision to discuss English writing in terms of its differences from Japanese writing showed that he was aware of the issue Aspinall (2003) raised as the first major reason why English education has been unsuccessful in Japan-that the disparity between the two languages is so great. Research in contrastive rhetoric between Japanese and English began with discussions of Japanese non-linear writing styles (Kaplan 1966) (2.2), which Mr. Doi supported by drawing a spiral shape on the board to describe Japanese writing (7.2.1). He explained that writing in English gets "to the point" (Mr. Doi, D class, May 9) as a way to encourage students to take a clear critical stance on the writing topic.

Like Mr. Doi in the D class, in the E class, Ms. Ellis displayed a certain sociopolitical aspect in her approach to the course with peer discussion tasks (6.2.1). Ms. Ellis was observed on numerous occasions promoting the benefits of peer reading to increase students' exposure to the different ways their classmates approached arguing. Through this process students developed a sense of an academic community in which they attempted to both persuade and meet the expectations of the peer group. This process (3.2.3) is integral to the development of writer identity according to a social constructivist perspective of L2 writing education (Scollon, 1991).

Peer reading as a concept was in fact observed at least once in each of the A-F classes, but what was notable was how different the approaches were, particularly in the Composition 1 classes. Ms. Ellis was consistent in the F class as she was in the E class, encouraging students' awareness of an academic community through collaborative learning activities where students worked together for mutual benefit. In contrast, Mr. Clark's application of peer reading was more of a group learning activity where students worked together for 
individual benefit (Bruffee, 1986) (3.2.3) and not their own, as it was the students' duty as a peer reader to "help" the writer (6.2.1).

Along the lines of the socially oriented view of writing in the sociopolitical approach, the genre discourse was observed in teachers' attempts to focus on the learners and their needs (Hyland, 2003) (2.1.6). This discourse was observed in the D class and the A and B classes. The genre discourse was applied in an attempt to increase students' awareness of critical argument as it is presented in different genres. Mr. Doi focused on it in the first semester in order to encourage students to grasp different ways of arguing. This concept was explained by Hyland (2008) (2.1.6) as a reader-oriented approach that raised students' awareness of the expectations within a particular genre.

Similarly, Ms. Aiba developed a reader-oriented approach in the second semester in the A and B classes through very specific genre-based tasks. Ms. Aiba explained in her interviews that she wanted the students to learn practical writing-the kind of writing they would need in the real world. This decision was based on the awareness that the students were not expected to do much academic writing as students in the English department.

The process discourse was observed in the C, D, E and F classes. The process approach in L2 writing education developed along with Communicative Language Teaching with a focus on the writing process as self-discovery, emphasizing creativity and fluency (Zamel, 1982; Hedgcock, 2005) (2.1.3). The observations of the process discourse involved a focus on the steps in the writing process such as brainstorming, outlining and revising (White \& Arndt, 1991; Tsui, 1996; Rinnert \& Kobayashi, 2001). The emphasis on these steps that included peer reading (6.2.1) was part of increasing students' consideration of their readers, making sure their ideas were complete, clear, and supported. The brainstorming step played a large part in the process approach in the C, D, E and F classes, with a great deal of focus on brainstorming techniques (7.1).

Finally, the skills discourse was observed in classes where the teachers focused on lexicogrammatical rules and essay structures and forms. This particular discourse would suggest a grammar-translation approach, but in the classes in which it was observed $(\mathrm{A}, \mathrm{B}, \mathrm{C}, \mathrm{E}$ and F), there was none of the traditional grammar-translation described by Matsuda (1997) 
and Poole (2005) (2.3.3) who suggested that the traditional grammar-translation method hindered the development of critical thinking. In the A and B classes, Ms. Aiba's first semester focus on grammar was meant to encourage students to think critically about the language, increasing their understanding of why English language grammar has certain rules. In the C, E and F classes, Mr. Clark's and Ms. Ellis's practices with language forms and essay structures was done, for the most part, in attempts to acknowledge, guide and persuade the reader (6.2).

To sum up, the current practices observed in the classrooms and discussed in the teacher interviews were various but purposeful. In relation to developing students' critical argument skills and writer identity, Mr. Doi and Ms. Ellis made conscious efforts to encourage critical thinking through in-class discussions of establishing and developing an argument. Ms. Aiba's and Mr. Clark's approaches were less direct, but they encouraged critical thinking through brainstorming activities. As for writer identity, Mr. Doi led discussions on cultural stereotypes to get students to consider the influence of their own identities on their writing. In his interview Mr. Doi explained that he held these discussions because he felt the issue was a big part of his own development as an academic writer in English, his second language. Ms. Ellis and Mr. Clark encouraged students to establish a position on a topic based on their own personal opinions and convictions, requiring students to consider their own identities. This point presents a contradiction to Ms. Ellis's explicit instruction that students were not to use personal language in their writing. Ms. Aiba's class, through the social practice discourse approach, required students to take on a variety of identities for different writing tasks. From political commentator to promoter, the students had to determine the appropriate voice for each task.

\subsubsection{Effects on students' writing}

The fifth research question was: How effective are these practices in terms of students' writing output? The instruments used in collecting data to answer this question included interviews with the students and the students' written texts, one from each student participant including argumentative essays ( $C$ and $F$ classes), research papers (D and E classes), and letters to the editor (A and B classes). The effectiveness of the teachers' practices was measured using an adapted Appraisal Theory framework (Martin, 1997, 
adapted using Bakhtin, 1986). This adapted framework allowed me to evaluate the lexical choices made by the students in their writing in relation to their writer identities (Ivanič, 1997) and this in turn allowed me to identify their writing game strategies (Casanave, 2003). In addition, students were asked to discuss their writing in interviews. It is important to note that students were not asked directly about writer identity or critical argument in their interviews in order to avoid skewing data. Instead, students offered their own ideas about these points through open discussions about their writing tasks.

As outlined in chapter 8, the students' writing displayed a certain ability to apply an appropriate writer identity, but often resorted to an authorial self, despite instructions from their teachers to write "objectively" and attribute their ideas to outside sources, more in line with a discoursal self. The effects of this seemed to be profound for some students. The expectations of the teachers did not seem to match the students' ability to meet them. On this point, Schneider and Andre (2007) (8.1.1) explain:

Students may convey their lack of identification with academic discourse through their misuse of citation conventions or specialized terminology or through their failed attempts at employing complex sentence structures in order to sound more academic" (para.5).

These features of the students' writing, along with those identified using the Appraisal Theory framework, revealed a struggle for the students with writer identity that was not addressed by teachers.

Ms. Aiba's assignment of writing a letter to the editor required a voice the students had very little experience with, that of a political commentator. The students seemed to have little awareness of this aspect of the task, focusing more on rhetorical persuasive techniques. Megumi's attempt at utilizing a political commentator voice was an attempt at displaying an authorial self (8.1.2). She used emotional language, but with a collective voice that she explained in her interview was intended to represent the common feeling of Japanese people, ultimately displaying an authorial-autobiographical self. The other student participants who did this task used an authorial voice and, like Megumi, used sympathy as the main rhetorical appeal. They used such ATTITUDE language as it is upsetting; outrageousness; selfishly; terrible; and lack of sense, displaying a distinct 
autobiographical self in addition to the authorial self.

Mr. Doi's and Ms. Ellis's argument essays required an academic voice with which students struggled, but seemed at least to understand, even if they could not achieve it. In Mr. Doi's class, Aya took on the challenge of writing about a topic with which she felt she identified, but not from within the same cultural community. Aya's attempts to write with an objective, discoursal self on the topic of policy making in the UK and the Islamic faith was beyond her ability (8.1.1). The result was a neutral argument that did not persuade her readers nor did it achieve the requirements of the task. Had she been able to write it using an authorial or autobiographical self, it may have been a more achievable task. Conversely, in the same class, Satoko chose a topic with which she had more familiarity, at least culturally, and found the task of writing objectively much more manageable. The three students in Ms. Ellis's Composition 2 class fell somewhere between Aya and Satoko. Nana chose a stock argument topic with little familiarity and struggled similarly to Aya. Ai's topic choice took a clear stance, but was not clearly arguable, as there was no opposing argument presented. Yuki chose a lesser-known topic, which she had a great deal of personal interest in and had accumulated a large amount of source evidence about. Yuki managed to use a discoursal self in her attributions to her sources, but resorted to an authorial self as a persuasive strategy.

Mr. Clark's assignments did not emphasize the use of a discoursal self, allowing the use of autobiographical supporting evidence on academic topics. He downplayed the element of voice or identity through very strict task parameters, leading to less confusion for the students about how best to approach the tasks in order to meet his expectations. Students contextualized their arguments in the local culture using relatively established culturally acceptable supporting ideas. The one student who used source evidence, Aki, explained that she did so because she had learned it in high school and thought it was an important persuasive technique. Yui, on the other hand, felt she was unable to use the skills she had learned in high school. She explained at the end of the year that she wrote according to Mr. Clark's instructions (8.1.2): 
I was just doing what [teacher] wanted us to do. Since we didn't have to use sources, I didn't use any. When he said "persuade your reader" I just used common sense for my argument. (Yui, January 22)

In evaluating the effect teachers' expectations and practices had on the students' writing, it seemed the request to write with an objective, discoursal self was mostly not achievable, as most students used an authorial self, or a combination of selves. In their interviews, most students complained about the struggle to understand how to meet the expectations of the tasks. However, in Mr. Clark's class, the students felt the expectations were clear, but not all expressed satisfaction with the effect this was having on the development of their writing skills. Yui explained that what she had learned was only how to write according to Mr. Clark's requirements.

These issues were raised in an evaluation of the writing game strategies utilized by the students, which were mostly consistent with the students' understanding of their teachers' expectations. These strategies included interacting with texts, blending voices, owning research experiences and telling a good story from them, speaking with authority, learning to love writing, and making the paper look right (Casanave, 2003). All six strategies were found among the students' selected texts, in varying degrees.

In interacting with texts, students practiced evaluation through peer reading exercises (8.2.1). They were encouraged to evaluate their own sources but ultimately displayed a lack of critical thought by not challenging or negotiating their use, an issue widely debated as it regards Japanese students' ability to think critically (2.2.2). In their interviews students explained that they felt they could not criticize sources on topics with which they were unfamiliar, further supporting Stapleton's (2001) point about the importance of students writing on familiar topics. As many students used a number of sources, the papers were comprised more of a high number of attributions, and less evaluation of them. Blending voices was a similar struggle for students who were unable to establish arguments on their own (8.2.2). Many resorted to "common sense" arguments (8.1.2) and others borrowed their arguments from their sources (3.5). Both Stapleton (2002b) and Masao (1976) suggested that mimicking writing patterns or borrowing arguments from sources are strategies used by EFL students to make their writing appear "native." 
A game strategy that particularly stands out is that of ownership of the research experiences and telling a good story from them. Most students were required to do at least some research for their writing tasks. Students in the D class experienced distinctly different senses of ownership through discussing their research with academics in the subject area (8.2.3). A strong connection between personal background and research topic meant a strong sense of ownership of the research for Satoko, but the weak connection for Aya meant there was little sense of ownership at all. This further echoes Stapleton's (2001) emphasis on the importance of topic familiarity for student writers (2.2.2). Students in the A, B, E and F classes all described varying degrees of ownership of their research. For some students this was because they had been practicing new writing skills such as essay structures (F class) or refutations (E class). Other students felt they had ownership of their research because they were required to work collaboratively and to present and defend their work to the rest of the class (A and B classes). In the C class, however, there were examples of frustration about a lack of ownership of the writing. This was particularly expressed by Yui who had earlier expressed dissatisfaction with a lack of identity in her own writing due to formulaic writing parameters set by her teacher (8.2.6). She explained, "It's not really your essay - it's all - you have to find a problem and fit into this space and stuff. So the style is - it's already made" (Yui, November 13).

The speaking with authority game strategy (8.1.4) proved to be less of a struggle for students than the discussion of Japanese students' critical thinking ability suggests (2.2.2). While some teachers and academics described Japanese students' as unable to speak with authority (Mack-Cozzo, 2002; Atkinson, 2002; Davidson, 1995), others claim that this is a misinterpretation due to the different approaches Japanese student writers take to constructing an argument (Kubota, 1999; Stapleton, 2002a). Several students described the issue of expressing an opinion as problematic only in making the opinion sound authoritative. Casanave (2002) asserts that students who become familiar with authorial voices can learn to mimic them. In terms of utilizing this game strategy, there was evidence that in every case where a thesis statement was provided, students were in fact speaking with authority. 
The remaining game strategies, learning to love writing, and making the paper look right, had everything to do with the students' feelings about the course and their teachers. Students felt more positive about their writing when they were given more tasks (8.1.5). In the $\mathrm{A}, \mathrm{B}$ and $\mathrm{C}$ classes the lesser amount of reading and writing expected led to some disinterest in or dissatisfaction with the writing tasks, whereas students in the D, E and F classes described more positive feelings about writing and their writing tasks, due to the heavier workload. This point strongly supports Arum and Roksa's (2011) (2.5) assertion that courses with more rigorous workloads are more appreciated by students and produce more improved results. As for making the paper look right, students in the C class discussed this point repeatedly in their interviews. Mr. Clark's rigid task parameters meant for all three student participants that meeting those requirements was the most important focus of the task. In all the other classes students struggled with this point as they felt unsure about their teacher's expectations (9.1.3).

In all, the six writing game strategies were all extremely relevant to the study, although not all were required for the assigned writing tasks. The game strategy of speaking with authority was often the default strategy when blending voices and owning the research were not achievable.

\subsubsection{Students' recognition of the focus issues}

The sixth and final research question was: Do students recognize critical argument and writer identity as important to their advanced writing education? The instrument used in collecting data to answer this question was the interviews with the students. Although never directly asked in their interviews, several students offered their own thoughts on issues related to critical argument and writer identity. The instances rose in discussions on the specific writing tasks assigned in their composition classes. In particular, the students in the $\mathrm{D}, \mathrm{E}$ and $\mathrm{F}$ classes clearly identified their own backgrounds as extremely relevant to their ability to argue on their topics. It is important to note here that these two teachers, Mr. Doi and Ms. Ellis, had explicitly instructed the students to write objectively, inevitably leading students to consider their own representations of themselves in their papers. 
As discussed in chapter 8, the students' topic choices for their research papers in the D, E and $\mathrm{F}$ classes caused them to think about why they chose the topic. In the D class, Aya struggled with her peers and teacher to justify her thesis. Because she did not have much familiarity with the culture of the topic, she recognized it as too big a challenge, and a disadvantage to her own writer identity. Aya chose the topic of a British student being forbidden to wear a veil to school (6.2.1). Although she had only limited familiarity with British culture as an exchange student there for one year, she had no background knowledge of Islamic culture, but felt she could successfully argue in favor of the girl wearing the veil to school from a non-Western perspective. The problem she faced was an inability to convince her peers of her position, which was counter to theirs. She commented, "It - oh, they're kind of thinking in the way - a way Western people does. So, it kind of has gap between me and them" (Aya, October 9). She recognized her identity as crucial to her thesis, and she tried to maintain her position. The point of being connected to the writing was very important to Aya, and although it was not something she wanted to risk in order to meet the expectations of her peers, ultimately she gave in to their expectations.

Also in the D class, Satoko's impression of the issues of identity and argument in her writing were not raised to the surface like they were with Aya since Satoko did not experience the same difficulties with her research paper. She was very confident in her topic. Her confidence came from the ability to build her thesis on a familiar topic that suited her own writer identity and was easily convincing for her peers.

In the E class, $\mathrm{Ai}$ and Nana struggled with the topics that they chose because they had strong feelings about them, but very little background. Nana struggled to use the teacher's feedback because she felt it was one-way communication with no room to negotiate and identify with the process (7.2.4). Ai had a different impression, feeling that she agreed with the teacher's feedback and appreciated the input. Yuki struggled with the specific instructions on constructing her thesis statement (7.2.1). Although Yuki managed to find a topic that she had both experience of and strong feelings about, she felt unsure about expressing her thesis the way her teacher wanted her to, feeling a disconnect and lack of her own identity in the particular style of writing. In the end she discovered that the 
identity she attempted to display was beyond her ability due to a lack of familiarity with the voice required to write on the subject.

As discussed in chapter 3, the study by Abasi et al (2006) examined students' written texts and interview data in an inquiry into students' awareness of identity construction and found that 'students' awareness of the intersection of writing and identity closely matche[d] their socialization into their prospective disciplines" (p.113). The current study echoes this in that the assignments students were required to write demanded at least some socialization into a particular discipline, dependent on the topic of the paper.

The socialization was developed through peer reading exercises and developed the students' sense of an academic community. The D class students most strongly experienced this through extensive peer work and interaction with an expert on their topics. The $\mathrm{E}$ and F class students had developed some sense through peer work, but felt they needed further expert assistance. The students in the A and B classes were exposed to a variety of disciplines, but without any interaction with others on their topics-peers or expertsthey were unable to socialize into the discipline and grasp the voice required to write in that discipline. Finally the $\mathrm{C}$ class students approached such socialization from a very fundamental level that was limited by peer reading tasks that were intimidating due to the expectation that students were required to "help" their peers (6.2.1) and by the very specific task parameters set by Mr. Clark.

To sum up, there was more recognition of writer identity and critical argument as important to the development of the students' writing in the cases where students struggled or feared a disconnection from their writing to meet the expectations of their teachers and/or peers. The standout examples were Aya (D), Nana (E), Yuki (E), and Yui (C), who all felt as though they sacrificed their identities in order to fit their writing into rigid task parameters or task expectations.

\subsubsection{Summary}

The six research questions revealed important considerations regarding Japanese university students' learning English academic writing in their compulsory English Composition courses. There is currently more discussion on the importance of 
communicative, critical writing practices in English writing education in Japan, and all four teachers agreed that current practices in university EFL writing should be understood as a communicative social act (Rinnert \& Kobayashi, 2001) (2.1.3). However, each teacher interpreted this differently. Mr. Doi encouraged students to consider how their identities culturally affected their writing. Ms. Ellis and Mr. Clark encouraged students to choose topics with which they felt a personal connection. Ms. Aiba set writing tasks that required students to take on a variety of writer identities.

The teachers' goals for the writing courses were for the most part practical. In terms of developing critical thinking skills as part of writing education, Mr. Doi considered this crucial. Ms. Ellis took a more creative approach, believing that students' freedom in choosing their research topics allowed them to develop their own understanding of the writing process. The other teachers' beliefs about writing revealed much more concern about accuracy and structure in writing, leading Ms. Aiba and Mr. Clark to focus more on grammar and forms of writing.

The identifiable cultural expectations of the teachers, like their goals, were dependent on their own identities, with the strongest influences coming from their postgraduate studies and their cultural backgrounds. Ms. Aiba and Mr. Clark both had postgraduate degrees in language education and used their theoretical knowledge to focus their courses on specific aspects of writing such as accuracy and structure. However, Ms. Aiba's course directed students toward practical writing needs, according to her understanding as a Japanese person of the needs of other Japanese people in Japanese society, whereas Mr. Clark's course directed students toward basic academic writing needs, according to his understanding as a Western teacher of Japanese students' needs in Japanese society. Mr. Doi and Ms. Ellis did not have a background in language education and therefore formed their expectations around their own personal writing experiences (Mr. Doi writing in English for his postgraduate theses in North America, and Ms. Ellis writing in Japanese for her postgraduate theses in Japan). These expectations were more sociopolitically oriented and focused more on critical thinking development and consideration of identity. 
The actual writing practices in these courses reflected the teachers' understanding of the general current practices in EFL writing education, and their own goals and expectations. Ms. Aiba's and Mr. Clark's focus on accuracy and forms meant their students wrote according to very specific guidelines. Ms. Ellis included some focus on accuracy and structure as well to an extent, but ultimately required students to write with fewer demands on forms, and instead focus more on meaning. Mr. Doi's belief that learning to write should develop critical thinking skills meant his students spent much of the class time working with peers and negotiating their ideas.

In terms of the effectiveness of the teachers' practices, very different results were revealed through the analysis of a selection of the students' papers using an adapted appraisal theory framework. Students often failed to meet teachers' expectations of writing objectively, resorting to an authorial self in their writing through the use of pathos-driven argumentation. The writing game strategy used often was speaking with authority, particularly when other strategies such as blending voices and owning research experiences was difficult to achieve.

The issue of ownership was a contentious one for several students that increased their awareness of their own writer identity and critical argument in writing. The sixth and final research question focused on this awareness, which most students discussed in their interviews without being prompted. The contentious aspect of the issue was that the students who were more acutely aware of their writer identity were made aware of it through the frustration of struggling to meet the expectations of their teachers' rigid task parameters. These students felt the rigidity of the tasks required them to risk a disconnection from their writing or abandon their own identities.

\subsection{Chapter summary}

This study set out to explore the situation in Japanese universities regarding students' learning of English writing. The focus issues of writer identity and critical argument came out of a review of literature on current issues in EFL writing education. In Japan, the current emphasis is on communicative writing and, to an extent, critical thinking skills 
development in order to stress sustainability of writing in English beyond the writing classroom.

This study involved observing the English Composition classes of four volunteer teachers and interviewing those teachers and two to four of their students in each class. The questions focused on current practices, goals, expectations, writing activities, effects on students' writing, and the students' awareness of the importance of the focus issues on their writing skills development. What was found was that teachers have different philosophies behind the decisions they make for their courses, but the classroom practices ultimately require students to consider their writer identities in the development of critical argumentation, even if the teachers reject the idea of discussing writer identity in class.

The students in the study responded differently to the teachers' approaches; some were satisfied with rigid, accuracy-focused writing tasks, and others preferred the freedom to decide on their own topics for researching and writing, and negotiating their ideas about their writing with their teachers and peers. There was some discrepancy between students' and teachers' beliefs about writing and learning to write, which led to some students' disinterest in learning.

The following chapter will conclude the thesis and provide a discussion on the implications of the findings of this study in order to make suggestions for future research in this area. As foundational skills students at the university level are expected to develop, much more attention needs to be given to writing and critical thinking education in Japan in order to improve what appears to be a too-widely varied writing curriculum. 


\section{Chapter 10. So what? Conclusion and possibilities for the future}

This study was designed to contribute to the field of EFL higher education composition studies by providing a rich description of the state of English language writing education at university level in Japan. Through an evaluation of relevant literature and an investigation into a Japanese university English department's compulsory English composition courses, the current study has provided a detailed description of what happens when Japanese students take English writing courses at a university in Japan before possibly heading to studies in higher education overseas. Taking into account previous relevant discussions, descriptions and interpretations along with the findings of this study, it should now be possible to assess the situation for the purposes of clarifying in what ways this thesis is in a position to make suggestions for pedagogical approaches to English composition in Japanese universities, and for future research.

This final chapter will conclude the thesis with a re-emphasis of the main findings and a summary of the significance and general and theoretical contributions of the study. This is followed by a discussion of the limitations of the research. Pedagogical recommendations for university English writing education in Japan are then made, followed by suggestions for areas of related future research. Numbers in parentheses (e.g. 2.2.2) indicate sections of the thesis where the issue was discussed.

\subsection{Main findings, significance and contributions of the study}

This study offers insight, from a social constructivist perspective, into the situation of learning English composition in a Japanese university, revealing a series of significant issues related to teachers' and students' approaches, expectations and outcomes in the writing courses. The intention was to provide a description of Japanese students' experiences learning English writing at university in Japan for the purposes of focusing on the relationship between what students did in their classes, how they felt about their classes, and what they produced in their writing tasks. This required an investigation into the teachers' philosophies behind designing their courses, as there were no prescribed guidelines. Therefore regular classroom observations, and teacher and student interviews 
were carried out over the yearlong courses. One final text from each student participant was selected for analysis.

\subsubsection{Findings}

This section will briefly summarize the findings according to the six research questions, in order.

1. What is the current practice of English writing education in relation to critical argument and writer identity in a Japanese university?

Taking critical argument and writer identity as focus points, the current practice of English writing education was investigated in terms of teachers' understanding and application of current approaches. Recent, relevant literature indicates that developments in English education in Japan are increasingly emphasizing communicative language education and critical thinking, but studies focusing on these aspects in university English writing education in Japan are lacking.

In their interviews, teachers explained their philosophies behind their approaches to the issues of communicative writing and developing critical thinking in the writing courses. All four teachers agreed that the students needed to learn to be aware of an audience when they write, and should learn to write for that audience. In terms of critical thinking teachers varied in their approaches. While Mr. Doi and Ms. Ellis felt it was important for the students to develop critical thinking skills in their writing education, Ms. Aiba felt students would benefit best from practical writing practices, and Mr. Clark avoided critical writing in order to keep students focused on specific writing conventions. This also applied to his reasons for not considering aspects of writer identity in the course. Writer identity, in fact, was a point none of the teachers felt they wanted to deal with in their courses. However, both Mr. Doi and Ms. Ellis, based on their own experiences of learning to write in a foreign language for their postgraduate studies, encouraged students to write impersonally and objectively, while still maintaining their honest feelings about their topics. Ms. Aiba rejected the idea of discussing writer identity in her course, yet she required students to write within different genre boundaries and conventions that ultimately required them to display different writer identities. 
2. What are teachers' goals for these writing courses?

The teachers' goals for the writing courses were very much dependent on their own backgrounds and experience, as there were no course guidelines or curriculum suggestions provided. Two criteria of Ivanič's (2004) Discourses of Writing Framework that were particularly revealing as they pertained to teachers' course goals were beliefs about writing and beliefs about learning to write. According to the course outlines collected from the teachers, the goals were numerous and varied. The goals varied from Ms. Aiba's practical writing skills, to Mr. Doi's and Mr. Clark's basic academic writing skills, to Ms. Ellis's formal, academic writing skills and attention to argumentation in Composition 1 and research in Composition 2. All four teachers agreed that students needed to learn fundamental conventions of writing, but varied greatly on the specifics of what those fundamental conventions were, including grammar of various levels, paragraphing, thesis statements, citations, essay parts such as introduction, body, conclusion, and basics such as capitalization, punctuation and margins.

Regarding critical argument and writer identity, information from course outlines showed that Ms. Ellis had intended to include arguing, persuading and thinking as goals, having included them in the fifteen course goals. Argumentative writing was also included in Mr. Doi's and Mr. Clark's course outlines. Writer identity, however, was rejected by the teachers as a course goal. It did not feature on any teacher's list of course goals, and in fact, Mr. Doi responded in his first interview that he had not thought of it.

\section{What are teachers' identifiable cultural expectations of EFL writing?}

In chapter 9 it was explained that the teachers' cultural expectations of EFL writing were identified in terms of their ideas about assessing students' writing. The feedback teachers gave was rather disparate. Ms. Ellis gave very extensive feedback, often covering papers in red pen, leaving students feeling appreciative, but also intimidated and overwhelmed. Mr. Doi's feedback was usually added to the extensive feedback from peers, but at least one student, Aya, was dissatisfied with this as she preferred less feedback from her peers and more from her teacher. Ms. Aiba's students generally did not appreciate her minimal feedback. Ms. Aiba's intention was to require more effort from the students in their 
revisions, but the minimal feedback only left them wanting more. Not all the student participants appreciated Mr. Clark's feedback, which focused more on format and language use and less on content; Yui was very dissatisfied. She wanted to write and receive feedback on her ideas. For all four teachers there was at least one student participant who was dissatisfied with the teacher's feedback. This showed an obvious discrepancy between the students' and teachers' expectations of EFL writing.

The students' acceptance or refusal of course materials and content was discussed in section 7.4. Students did not necessarily understand or agree with their teachers' expectations of their writing output, and the often laborious in-class practices designed to prepare them for the specific writing tasks, particularly those of the native speaker teachers. Mr. Clark and Ms. Ellis explained that the pace was a matter of the students' ability to grasp the specific skills teachers felt the students needed to have in order to complete the assigned writing tasks. Many students complained of the in-class practices or the slow pace at which their teachers took them through fundamental skills, sometimes spending weeks on one aspect such as thesis statements.

4. What practices do teachers bring to the classroom to develop critical argument and writer identity in students' writing?

As for the actual practice tasks students did in their writing courses, teachers rejected the idea of focusing on writer identity in their courses, and had different ideas about the importance of working with argumentation, although three of the four teachers assigned an argumentative writing task. It was discovered through generated theory that in terms of critical argument and writer identity, teachers focused students for the most part on awareness of the reader, and development of the thesis. Having identified the teachers' different discourses of writing approaches (Ivanič, 2004), it was possible to discuss how the aspects of critical argument and writer identity played a part in the classroom practices.

Argument and identity were developed with varying degrees of emphasis in the observed courses. Although the teachers rejected the idea of teaching writer identity, three teachers assigned tasks that required students to represent themselves in their writing in specific 
ways, while Mr. Clark put less emphasis on this point allowing students to use personal language and not requiring them to use any outside sources in building their essays. Ms. Aiba's social practices discourse approach involved assigning a variety of writing tasks, most outside the academic realm, requiring students to use genre-appropriate voices. Mr. Doi and Ms. Ellis both encouraged students to write objectively, but to maintain personal involvement in the writing. Students were required to use outside sources to attribute their ideas. The students were regularly reminded to think about their readers and the importance of persuading them with information from sources. Ms. Ellis put less emphasis on this point in her Composition 1 course as she felt the basic structure of the essay was more significant than the source of ideas. Argument was focused on more directly in the courses where argumentative writing was taught, with teachers spending significant class time on students' development of their thesis.

\section{How effective are these practices in terms of students' writing output?}

Through an analysis of a selection of the students' written texts that focused on aspects of writer identity including Clark and Ivanič's (1997) possibilities for selfhood, the number of appraisal words students used, and the writing game strategies used, it was discovered that teachers' emphasis on the communicative and argumentative aspects of writing had an effect on students' writing, but this was not always positive. Students struggled with their teachers' expectations of their writing, attempting to use an appropriate voice or self as they understood it to be required for a particular task. Students' attempts to attribute their ideas to sources were indicative of a discoursal self, one appropriate for objective, academic writing. But all of Mr. Doi's and Ms. Ellis's students who attributed their ideas to sources also judged those ideas, displaying more of an authorial self than a discoursal one. Most students were able to take a strong authorial stance on their topics, with the exception of Aya whose neutral stance was the result of trying to meet the expectations of her peers. For Ms. Aiba's assignments that required different voices, students struggled to find an appropriate self-representation in their writing. For Mr. Clark's assignments, students felt confident they were meeting the teacher's expectations, as they were very specific and did not require any outside sources. 
6. Do students recognize critical argument and writer identity as important to their advanced writing education?

The study showed that most students did focus on argumentation, and that writer identity was a central element, even though students may not have been aware of it as it was not addressed directly in discussions in classes or interviews. In their interviews, most students discussed the struggle of expressing their opinions in their writing. This was particularly the case for students who were required to write a persuasive, argumentative paper. To improve one's ability to express ideas in English writing remained the most commonly discussed goal, although the difficulty with expressing ideas was more often attributed to poor vocabulary. It was interesting that some students raised the issue of identity due to their choice of topic. The case studies of Aya and Ai, highlighted in chapter 8, both revealed that identity was important to their research topics. Aya chose to write about cultural identity conflict between a Muslim girl and her English school uniform rules, often discussing the point that her own identity was compromised in the development of the argument in order to meet the expectations of her peers. Ai chose the topic of sign language education, emphasizing as part of her argument the pride of deaf people who have identities of their own as non-hearing people. The argument relied on this point to support her thesis that sign language education needed to be increased in Japan. The other interesting case related to this point is Yui's, in which she described a distinct lack of ownership of, or identification with, her writing. She felt that she had compromised her identity in order to meet the specific guidelines of the writing task.

\subsubsection{Significance and contribution of the study}

The main contribution of the study is the rich description of what actually happens in English writing courses in a university in Japan from the social constructivist perspective of a Western researcher. From the literature review it was clear that this area of inquirylearning university EFL writing in a non-English medium context-lacked a qualitative, exploratory study of university EFL writing students in situ. Although some studies were conducted with Japanese students in English-medium high schools overseas (e.g. Inoue, 1997; McFreely, 1999) and universities (e.g. Spack, 1997; Yasuda, 2005), there were few in-

depth studies conducted with Japanese students in Japan. In addition, as Japanese 
university students develop more global identities, and as Japanese universities look more toward increasing international student numbers (1.1), it is in the better interest of those universities to offer curricula with more global appeal and to meet the ever-changing cultural expectations of the students. Therefore this study helps to provide some suggestions in developing such curricula.

A significant contribution of this study may be the focus on cultural expectations of both the students and teachers, particularly revealed in this study by the two main focus areas of writer identity and critical argument. As discussed in relation to research question 3 on the teachers' identifiable cultural expectations of EFL writing, a great disparity was found among the teacher participants, although this was not dependent on teachers' background or identity. A disparity was also evident between the expectations of the teachers and their students. With no guidelines for the courses, the teachers had to design their own course objectives and goals based on their own beliefs about learning to write. These beliefs were built on their personal backgrounds, mostly of either learning to write in a foreign language themselves, or of their past experience teaching English Composition in Japan. The result was rather different courses, with very different expectations. The students placed in the same course had different ideas about what to expect from the course, but initially felt satisfied with their teacher's plan. As the course went on, students became dissatisfied, often wishing to "write more" and focus more on ideas rather than format or grammar.

In addition, cultural expectations were further revealed in the analysis of the students' acceptance or refusal of course materials and teachers' approaches, discussed in section 7.4. The teacher who felt the least confident about teaching English Composition, Mr. Doi, was most encouraging of the students' critique of course materials. He encouraged the students to be "critical" and "angry" in order to develop their critical thinking. Ms. Ellis used her first two years experience of teaching her courses to place more control over her courses and leave less time for critique, although she regularly encouraged students to participate in critiquing each other's writing, often on the board in front of the whole class. Ms. Aiba and Mr. Clark's highly controlled courses left very little room for students' 
critique, which caused most of their students to complain about the courses in their interviews.

\subsubsection{Theoretical contributions}

The application of Appraisal Theory within Systemic Functional Linguistics (Martin, 1997; 2000) in this study reveals that as a research framework it is flexible and can be adapted to focus on specific aspects, such as writer identity and critical argument. By adding Clark and Ivanič's (1997) possibilities of selfhood in order to identify the different selves students displayed in their writing, and Casanave's (2002) writing game strategies to reveal the students' attempts to meet the task expectations, it became clear that by highlighting the specific lexicogrammatical choices that fall within the linguistic categories of ATTITUDE, ENGAGEMENT and GRADUATION, students' writer identities could be directly linked with their attempts to argue in their papers.

The link between students' identities and their attempts to argue, outlined in Figure 1 in chapter 3 , is an important one as it takes into consideration the social nature of identity construction and the contributing cultural and intercultural factors. Establishing appropriate academic writer identities by building on a cultural identity is key to succeeding in the cross-cultural construction of critical argument. As discussed in section 3.4, students start to construct their identities through social interaction, and establishing a cultural identity happens within a group, known as a "collective identity" (Gomez-Estern, et al., 2010, p.233). The concept of a collective identity is an important consideration in this study in that student writers learned to conform to the group, in this case their classmates (discussed in section 6.2), and having achieved that they could build a socio-cultural identity as a member of the group. I believe this process of identity construction, and the understanding of it, is required before the student writer can build an academic writer identity.

\subsection{Limitations}

As a largely qualitative study, there is the risk of relying too much on impression, and less on trialed and tested formulas. Also, as a study that focuses on cultural differences, it is 
important to consider the inevitable bias of approaching the issue from one of the cultures involved in the study.

First, the impressionistic nature of this study is a point that needs to be clarified. Because results of text analysis studies have mostly been impressionistic (4.3.3.1), researchers have attempted to provide more in-depth, controlled analysis with less emphasis on researcher intuition (Schilperoord, 1996). However, as Sander and Schilperoord (2006) explained, with an appropriate framework for text analysis, psycholinguistic processes involved in language production can be identified. This is why I chose Appraisal Theory to provide the base framework, as it stipulates very specific guidelines for specific groups of words or phrases. Also, it can be adapted to offer more specifically detailed analysis of language use, such as writer identity and writing game strategies.

Second, in chapters 1 and 4, I discussed the potential limitation of being a Western researcher in the Japanese context and the possibility of my cultural position invalidating the study. I explained the impact of the socio-cultural environment on students' exposure to L2 writing and discussed the congruence of the students', teachers' and my sociocultural positionalities as an aspect that could invalidate the study (4.1). What I have done to counter this potential invalidation is to expose my own subjectivity and to explore the influence of it on my data, establishing my position as an outsider as an advantage to the study. Social constructivist theory asserts that people's ideas coincide with their experiences (3.2). With this understanding, writers build on their socio-cultural awareness, a key point in identity construction. Therefore through my subjective position as a Western researcher, I was able to observe the students' attempts at constructing an identity within the Western cultural context of English composition.

\subsection{Pedagogical concerns and future research}

This thesis serves to inform pedagogic practices of English academic writing at the university level in Japan. There are a number of concerns regarding the specific context of this study, especially the inconsistencies between courses due to the fact that there were no guidelines from the university or department. As for future research, having addressed the research questions for this study, some further questions are raised. 


\subsubsection{Pedagogical recommendations}

Some obvious pedagogical concerns raised by this study include the lack of standardized course objectives and goals, and the lack of any suggested objectives and goals or any real form of evaluation or academic review from the university. A significant consideration on this point is that this university in Japan essentially has a "clean slate," meaning the professional development of its university teachers can be established without having to deal with any problems with systems already in place. Professional development needs to be done in a way to first provide university teachers with a standardized set of objectives and goals for their courses, and then provide suggestions for ways of reaching those goals and evaluating the outcomes. Although each teacher in this study had established their own set of objectives and goals, at least one student, Yui, expressed frustration with the differences between courses with the same title, feeling it was unfortunate to be assigned to a particular teacher for her compulsory writing course (8.3.4). Finally, the university would benefit from establishing an academic review process in order to help guide teachers and offer a more improved program.

Regarding course content, students need to be assigned more reading tasks as part of their writing. Reading gives students necessary exposure to the target language and provides opportunities to discuss other writers' rhetoric and approaches to writing. The students in this study who did more reading were in Mr. Doi's and Ms. Ellis's classes. Although all of those students may not have been successful in taking the best advantage of their reading tasks, they were still required to consider aspects of the readings when constructing their

essays. In addition, they were required to read further for research purposes. Arum and Roksa (2011) suggest a minimum of 40 pages of reading per week and 20 pages of writing per semester (not specifically for writing courses, but for university courses in general) in order to improve students' writing and reasoning ability (2.5). Although the context for this recommendation is for students in the US, reading and writing in their native language of English, there is certainly some merit in applying this recommendation to Japanese students of English. A general way of applying this recommendation is to include reading tasks as part of the compulsory writing courses. Teachers can provide students with model essays for discussion and from which students can adopt certain writing strategies, similar 
to the practices of Mr. Doi and Ms. Ellis. It is essential for teachers to teach students how to read for their writing courses. In the case of lower level proficiency students, where the focus is more on skills, the amount and type of reading assigned must be adjusted accordingly.

Finally, rather than teaching students to write objectively, we should be teaching them how to write authoritatively, and to own their research. Hyland (2002b) recommends that rather than teaching students to write impersonally, they should be taught how different disciplines approach academic writing in order to attain subject-specific options for academic writing $(2.2 .2,7.3 .3)$. Hyland points out that English L2 students tend to write better using an active voice. He admits that at the introductory level, particularly for students with lower level proficiency in the target language, teaching students impersonal writing can be useful in reducing overuse of personal pronouns, but believes it is better to show students when personal pronouns can be used appropriately. Based on the results of this study, it seems clear that the students would have benefitted more from the approach Hyland (2002b) describes, allowing students with similar or higher levels of English language proficiency to better understand how, in what ways and to what extent their writing can be personal, dependent on the subject-specific area in which they are writing.

\subsubsection{Suggestions for future research}

An important question at this stage is: In answering the research questions, what additional questions have been raised? The research questions were answered using a thorough analysis of observation data and students written texts, with supporting data from the interviews. The theories that emerged from the data-audience awareness and thesis development-showed that the pre-selected focus areas of critical argument and writer identity were appropriate. Also, the research question on teachers' cultural expectations of EFL writing proved to be particularly valuable, as it was an important aspect of the study to establish the relationship between my socio-cultural position and those of the students and teachers. With the specific focus of students' learning experiences on these theories, the answers to the research questions raised some additional questions that serve as suggestions for future research. 
Regarding the contribution of the study to insights about cultural expectations (10.1.2), it seems differences between NNS and NS teachers' teaching philosophies and approaches should be a focus for future research. While Poole $(2005 ; 2010)$ has discussed the issue, the point of NNS and NS teachers in this study working at different paces has not been addressed in in-depth studies. It would be of value to explore possible trends in notable differences between NNS and NS teachers' beliefs about the pace at which they believe Japanese students can learn English writing at university.

In addition, further attention should be given to particular identity variables as they affect culture. For example, while an individual's writing style is molded by socio-cultural tendencies and pre-formulated schemata, gender may also have implications when studying the development of a particular writing style or tendency (2.2.2). This particular issue is pertinent to studies like this, involving mostly female students whose L1 is Japanese, as the female role as subordinate to that of the male role permeates the culture and language. Gender is just one of many potentially significant identity variables that could be investigated further.

\subsection{Evaluation of future effect of the study}

Changes in education systems can be difficult in any cultural context. In Japanese universities, there is a long tradition of university teachers maintaining autonomy and not sharing classroom practices. However, as Japan faces decreasing numbers of Japanese students, in order to keep its institutions of higher learning open, an increasing number of students will need to be brought in from overseas. Because of this need, it is at this time that university policy makers are looking at what changes can be made. This study has revealed a number of suggestions for a university writing program from an outsider's perspective. As resistant as traditional university systems in Japan may be, it is inevitable that programs offering more global appeal will be developed. I believe a foundation course such as English Composition will be carefully scrutinized by teachers and curriculum developers who will agree that standardizing goals, increasing reading tasks, and teaching students to write authoritatively will be effective changes to implement. 


\section{References}

Abasi, A.R., Akbari, N., Graves, B. (2006). Discourse appropriation, construction of identities, and the complex issue of plagiarism: ESL students writing in graduate school. Journal of Second Language Writing 15.2, 102-117.

Anthias, F. (2002). Where do I belong? Narrating collective identity and translocational positionality. Ethnicities, 2(4), 491-514.

Anthony, L. (2000). Implementing genre analysis in a foreign language classroom. TESOL Matters, 10(3), 18-24.

Arum, R. \& Roksa, J. (2011). Academically adrift: Limited learning on college campuses. Chicago, IL: University of Chicago Press.

Aspinall, R.W. (2003). Japanese nationalism and the reform of English language teaching. In R. Goodman, and D. Phillips (Eds.), Can the Japanese change their education system? (pp.103-118), Wallingford, CT: Symposium Books.

Atherton, J.S. (2011). Learning and Teaching; Resistance to Learning.7 pars., Retrieved from http://www.learningandteaching.info/learning/ resistan.htm

Atkinson, P. \& Hammersley, M. (1994). Ethnography and participant observation. In N.K. Denzin and Y.S. Lincoln (Eds.), Handbook of Qualitative Research (pp. 248-261). Thousand Oaks, CA: Sage Publications.

Atkinson, D. (1997). A critical approach to critical thinking in TESOL. TESOL Quarterly, 31(1), 71-94.

Atkinson, D. (2001). Reflections and Refractions on the JSLW special issue on voice. Journal of Second Language Writing, 10(1-2), 107-124.

Atkinson, D. (2003). Writing and culture in the post-process era. Journal of Second Language Writing, 12(1), 49-63.

Atkinson, D. (2005). Situated qualitative research and second language writing. In P. K. Matsuda \& T. Silva (Eds.), Second language writing research: Perspectives on the process of knowledge construction (pp. 49-64). Mahwah, NJ: Lawrence Erlbaum.

Bakhtin, M.M. (1986). Speech genres and other late essays. Edited by M. Holquist, translated by V.W. McGee. Austin: University of Texas Press.

Ballard, B. \& Clanchy, J. (1991). Teaching students from overseas: A brief guide for lecturers and supervisors. Sydney, Australia: Longman Cheshire. 
Bawarshi, A.S. (1997). Beyond dichotomy: Toward a theory of divergence in composition studies. Journal of Advanced Composition, 17, 69-82.

Belcher, D. (2004). Trends in teaching English for specific purposes. Annual Review of Applied Linguistics, 24, 165-186.

Benesch, S. (1991). ESL on campus: Questioning testing and tracking policies. In. S. Benesch (Ed.), ESL in America: Myths and possibilities (pp. 59-74). Portsmouth, NH: Boynton/Cook.

Benesch, S. (1993). Critical Thinking: A Learning Process for Democracy. TESOL Quarterly, 27(3), 545-548.

Benesch, S. (2001). Critical English for Academic Purposes: Theory, Politics and Practice. Mahwah, NJ: Lawrence Erlbaum.

Bereiter, C. \& Scardamalia, M. (1983). Does learning to write have to be so difficult? In A. Freedman, I. Pringle \& J. Yalden (Eds.), Learning to write: First language/second language. Applied linguistics and language studies series (pp.20-23). London, UK: Longman.

Bereiter, C. \& Scardamalia, M. (1987). The psychology of written composition. Hillsdale, NJ: Erlbaum.

Berger, P. \& Luckmann, T. (1966). The social construction of reality. London, UK: Allen Lane.

Bhatia, V.K. (1993). Analyzing genre: Language use in professional settings. London, UK: Longman.

Bhatia, V.K. (2002). Applied Genre Analysis: Analytical Advances and Pedagogical Procedures. In A.M. Johns (Ed.) Genre in the Classroom: Multiple Perspectives (pp.279-284). Mahwah, NJ: Lawrence Erlbaum.

Bizzell, P.L. (1978). The Ethos of Academic Discourse. College Composition and Communication, 29(4), 351-355.

Bizzell, P.L. (1982). Cognition, convention, certainty: Knowledge about writing, Pre/Text, 3, 213-243.

Bowen, G.A. (2006). Grounded Theory and Sensitizing Concepts. International Journal of Qualitative Methods, 5(3), 1-9.

Bruffee, K. (1986). Social construction, language, and the authority of knowledge: A bibliographical essay. College English, 48, 773-790.

Bruner, J. (1966). Toward a Theory of Instruction. Cambridge, MA: Harvard University Press. 
Butler, Y.G. (2007). Foreign language education at elementary schools in Japan: Searching for solutions amidst growing diversification. Current Issues in Language Planning, 8(2), 129-147.

Byrd, P. (2005). Instructed Grammar. In E. Hinkel (Ed.) Handbook of Research in Second Language Teaching and Learning (pp.545-562). Mahwah, NJ: Lawrence Erlbaum.

Cain, M.A. (1995). Revisioning Writers' Talk: Gender and Culture in Acts of Composing. Albany, NY: State University of New York Press.

Canagarajah, A.S. (2002). Critical academic writing and multilingual students. Ann Arbor, MI: The University of Michigan Press.

Carroll, T. (1997). From script to speech: Language policy in Japan in the 1980s and 1990s. Nissan Occasional Paper Series.

Carson, J.G. \& Nelson, G.L. (1994). Writing Groups: Cross-Cultural Issues. Journal of Second Language Writing 3(1), 17-30.

Carspecken, P.F. (1996). Critical Ethnography in Educational Research: A Theoretical and Practical Guide. New York: Routledge.

Carter, R. \& Nunan, D. (Eds.).(2001). The Cambridge guide to teaching English to speakers of other languages. Cambridge, UK: Cambridge University Press.

Casanave, C.P. (2002). Writing games: Multicultural case studies of academic literacy practices in higher education, Mahwah, NJ: Lawrence Erlbaum.

Casanave, C.P. (2003). Looking ahead to more sociopolitically-oriented case study research in L2 writing scholarship. Journal of Second Language Writing, 12, 85-102.

Casanave, C.P. (2010). Taking risks?: A case study of three doctoral students writing qualitative dissertations at an American university in Japan. Journal of Second Language Writing, 19(1), 1-16.

Chandrasoma, R., Thompson, C. \& Pennycook, A. (2004). Beyond Plagiarism: Transgressive and Nontransgressive Intertextuality. Journal of Language, Identity \& Education, 3(3), 171193.

Chapman, M. (2004). An Interview with Kazuhiko Saito. Shiken: JALT Testing \& Evaluation SIG Newsletter, 8(2), 10-12.

Clark, R., \& Ivanič, R. (1997). The Politics of Writing. New York: Routledge.

Cohen, L., Manion, L., \& Morrison, K. (2007). Research Methods in Education (6 $6^{\text {th }}$ ed.). New 
York: Routledge.

Cohen, L., Manion, L., \& Morrison, K. (2011). Research Methods in Education ( $7^{\text {th }}$ ed.). New York: Routledge.

Connor, U. (2003). Changing currents in contrastive rhetoric: Implications for teaching and research. In B. Kroll (Ed.), Exploring the dynamics of second language writing (pp.218-241). New York: Cambridge University Press.

Costino, K.A. \& Hyon, S. (2011). Sidestepping our "scare words": Genre as a possible bridge between L1 and L2 compositionists. Journal of Second Language Writing 20, 24-44.

Cotterall, S. \& Cohen, R. (2003). Scaffolding for second language writers: producing an academic essay. ELT Journal 57(2), 158-166.

Creswell, J.W. (2009). Research Design: Qualitative, Quantitative, and Mixed Methods Approaches. Thousand Oaks, CA: Sage.

Crotty, M. (1998). The foundations of social research: Meaning and perspective in the research process. Australia: Allen and Unwin.

Daniels, H. (2006). The 'Social' in Post-Vygotskian Theory. Theory and Psychology 16(1), $37-$ 49.

Davidson, B. (1995). Critical Thinking Education Faces the Challenge of Japan. Inquiry: Critical Thinking Across the Disciplines, 14(3), 31 pars. Retrieved from http://www.shss. montclair.edu/inquiry/spr95/davidson.html

Davies, R.J. \& Ikeno, O. (2002). The Japanese Mind: Understanding Contemporary Japanese Culture. North Clarendon, VT: Tuttle Publishing.

Davies, R. (1999). A survey of Japanese and English composition instruction in Japan. Paper presented at the 12th AILA Congress. Waseda University, Tokyo, Japan.

Davies, W. (2003). A cautionary note about the teaching of critical reasoning. In Learning for an Unknown Future, Proceedings of the 26th HERDSA Annual Conference, Christchurch, New Zealand, 134 pages, Retrieved from http://www.herdsa.org.au/wp-content/uploads/ conference/2003/papers/HERDSA70.pdf

Deckert, G. (1993). Perspectives on plagiarism from ESL students in Hong Kong. Journal of Second Language Writing, 2, 131-148.

Denzin, N. K. (2009). The Research Act: A Theoretical Introduction to Sociological Methods. Piscataway, NJ: Aldine Transaction.

Doi, T. (2001). The Anatomy of Dependence (special edition). Tokyo, Japan: Kodansha. 
Donato, R. (1994). Collective scaffolding in second language learning. In J.P. Lantolf and G. Appel (Eds.), Vygotskian Approaches to Second Language Research (pp.33-56). Westport, CT: Ablex Publishing.

Duff, P. \& Uchida, Y. (1997). The negotiation of teachers' sociocultural identities and practices in post-secondary EFL classrooms. TESOL Quarterly 31,451-486.

Duppenthaler, P.M. (2002). The Effect of Three Types of Written Feedback on Student Motivation. JALT Journal, 24(2), 130-154.

Duppenthaler, C., Viswat, L., and Onaka, N. (1989). Learning Strategies of Japanese Learners of English. In V. Bickley (Eds.), Language Teaching and Learning Styles Within and Across Cultures, (pp.90-99), Institute of Language in Education: Hong Kong Education Department.

Dyer, B. \& Friederich, L. (2002). The personal narrative as cultural artifact: Teaching autobiography in Japan. Written Communication, 19(2), 265-296.

Edge, J. \& Richards, K. (1998). May I See Your Warrant, Please?: Justifying Outcomes in Qualitative Research. Applied Linguistics 19(3), 334-356.

Eemeren, F.H. van, \& Grootendorst, R. (1984). Speech acts in argumentative discussions: A theoretical model for the analysis of discussions directed towards solving conflicts of opinion, Dordrecht, The Netherlands: Floris Publications.

Eemeren, F.H. van, \& Grootendorst, R. (1992). Argumentation, communication, and fallacies: A pragma-dialectical perspective. Hillsdale, NJ: Lawrence Erlbaum.

Eisner, E. (2003). Concerns and aspirations for qualitative research in the new millennium. In N. Addison \& L. Burgess, Issues in Art and Design Teaching, (pp.52-60). New York: Routledge Farmer.

Ellis, R. \& Barkhuizen, G. (2005). Analyzing Learner Language. Oxford, UK: Oxford University Press.

Ennis, R.H. (1998). Is critical thinking culturally biased? Teaching Philosophy, 21, 15-33.

Eschholz, P.A. (1980). The prose models approach: Using products in the process. In T.R. Donovan \& B.W. McClelland (Eds.), Eight approaches to teaching composition. Urbana, IL: National Council of Teachers of English.

Fairclough, N. (1992). Discourse and social change. Cambridge, UK: Polity Press.

Flower, L. (1979). Writer-Based Prose: A Cognitive Basis for Problems in Writing. College English, 41(1), 19-37. 
Flowerdew, J. (2005). A Multimethod Approach to Research Into Processes of Scholarly Writing for Publication. In P. K. Matsuda \& T. Silva (Eds.), Second language writing research: Perspectives on the process of knowledge construction, (pp. 65-78). Mahwah, NJ: Lawrence Erlbaum.

Fotos, S. (2005). Traditional and grammar translation methods for second language teaching. In E. Hinkel (Ed.), Handbook of research in second language teaching and learning (pp. 653-670). Mahwah, NJ: Lawrence Erlbaum.

Fowler, F.J. Jr. (2002). Research Methods (3rd ed.). Newbury Park, CA: Sage.

Fox, H. (1994). Listening to the World: Cultural Issues in Academic Writing. Urbana, IL: National Council of Teachers of English.

Frager, R. (1970). Conformity and anticonformity in Japan. Journal of Personality and Social Psychology, 15(3), 203-210.

Gay. L. (2010). Grounding the JET: The exchange program faces a do-or-die moment. Metropolis, 866, 11 pars., Retrieved from http://metropolis.co.jp/features/the-last-word/ grounding-the-jet/

Gilfert, S. (1999). Let's Write In English: Teacher, We Never Learned That. The Internet TESL Journal, 5(4), 22 pars., Retrieved from http://iteslj.org/Articles/Gilfert-LetsWrite. html

Glaser, B.G. (1978). Theoretical sensitivity: Advances in the methodology of grounded theory. Mill Valley, CA: Sociology Press.

Glaser, B.G. with the assistance of Holton, J. (2004). Remodeling Grounded Theory. Forum Qualitative Sozialforschung / Forum: Qualitative Social Research, 5(2), Art. 4, 80 pars., Retrieved from http://www.qualitative-research.net/index.php/fqs/article/view/607/ 1315

Glaser, B.G. \& Strauss, A.L. (1967). The discovery of grounded theory: Strategies for qualitative research. Chicago, IL: Aldine.

Gómez-Estern, B.M., Amián, J.G., Sánchez Medina, J.A., \& Marco Macarro, M.J. (2010). Literacy and the Formation of Cultural Identity. Theory Psychology 20(2), 231-250.

Gorsuch, G.J. (1999). Exploring the relationship between educational policy and instruction in Japanese high school EFL classrooms. PhD dissertation, Temple University, Tokyo, Japan.

Greene, S. \& J. M. Ackerman. (1995). Expanding the constructivist metaphor: A rhetorical perspective on literary practice. Review of Educational Research 65(4), 383-420.

Halliday, M.A.K. (1978). Meaning and the construction of reality in early childhood. In H.L. 
Pick \& E. Salzman (Eds.), Modes of perceiving and processing of information (pp. 67-96). Hillsdale, NJ: Erlbaum.

Halliday, M.A.K. (1985). An Introduction to Functional Grammar. London, UK: Arnold.

Halliday, M.A.K. (1994). Language as Social Semiotic. In J. Maybin (Ed.) Language and literacy in social practice: a reader (pp.23-43). Clevedon, UK: Multilingual Matters.

Hamp-Lyons, L. (2007). The Impact of Testing Practices on Teaching Ideologies and Alternatives. In J. Cummins \& C. Davison (Eds.) International Handbook of English Language Teaching (Vol. 1, pp. 487-504). Norwell, MA: Springer.

Harre, R. (1984). Personal Being: A theory for individual psychology. Cambridge, MA: Harvard University Press.

Heard, M. (2008). What Should We Do with Postprocess Theory? Pedagogy, 8(2), 283-304.

Hedgcock, J. (2005). Taking stock of research and pedagogy in L2 writing. In E. Hinkel (Ed.), Handbook of research in second language teaching and learning, (pp.597-613). Mahwah, NJ: Lawrence Erlbaum.

Helms-Park \& Stapleton, P. (2003.) Questioning the importance of individualized voice in undergraduate L2 argumentative writing: An empirical study with pedagogical implications. Journal of Second Language Writing, 12(3), 245-265.

Henrichsen, L.E. (1989). Diffusion of innovations in English language teaching: The ELEC effort in Japan, 1956-1968. New York: Greenwood Press.

Hinds, J. (1983). Contrastive Rhetoric: Japanese and English. Text, 3(2), 183-195.

Hinds, J. (1985). Misinterpretations and common knowledge in Japanese. Journal of Pragmatics 9(1), 7-19.

Hinds, J. (1990). Inductive, deductive, quasi-inductive: Expository writing in Japanese, Korean, Chinese and Thai. In: U. Connor \& A. Johns, (Eds.), Coherence in writing: Research and pedagogical perspective (pp.87-110), Alexandria, VA: TESOL.

Hirose, K. (1998). The effects of English paragraph writing instruction on Japanese university students. JACET Bulletin, 29, 51-64.

Hirose, K. (2001). Persuasive writing in L1 and L2: A look at Japanese EFL students' rhetorical organization strategies. JACET Bulletin, 33, 43-56.

Hirose, K. (2003). Comparing L1 and L2 organizational patterns in the argumentative writing of advanced Japanese EFL students. Journal of Second Language Writing, 12, 181209. 
Hirose, K. (2005). Product and process in the L1 and L2 writing of Japanese students of English. Hiroshima, Japan: Keisuisha.

Hirose, K. \& Sasaki, M. (1994). Explanatory variables for Japanese students' expository writing in English: An exploratory study. Journal of Second Language Writing, 3, 203-229.

Hirvela, A. \& Belcher, D. (2001). Coming back to voice: The multiple voices and identities of mature multilingual writers. Journal of Second Language Writing, 10 (1-2), 83-106.

Holliday, A.R. (1994). Appropriate Methodology and Social Context. Cambridge, UK: Cambridge University Press.

Holstein, J.A. \& Gubrium, J.F. (1995). The Active Interview. Thousand Oaks, CA: Sage.

Hood, S. (2004). Appraising Research: Taking a stance in academic writing. PhD thesis, University of Technology Sydney, Australia.

Hung, H.L. \& Hyun, E. (2010). East Asian international graduate students' epistemological experiences in an American University. International Journal of Intercultural Relations 34, 340-353.

Hunston, S. \& Thompson, G. (Eds.). (2000). Evaluation in Text. Oxford, UK: Oxford University Press.

Hyland, K. (2002a). Authority and invisibility: authorial identity in academic writing. Journal of Pragmatics, 34(8), 1091-1112.

Hyland, K. (2002b). Options of identity in academic writing. ELT Journal, 56(4), 351358.

Hyland, K. (2003). Second Language Writing. Cambridge, UK: Cambridge University Press.

Hyland, K. (2005). Digging up texts and transcripts: Confessions of a discourse analyst. In P. K. Matsuda \& T. Silva (Eds.), Second Language Writing Research: Perspectives on the process of knowledge construction (pp. 177-189). Mahwah, NJ: Lawrence Erlbaum.

Hyland, K. (2006). English for Academic Purposes: An Advanced Resource Book. New York: Routledge.

Hyland, K. (2008). Writing Theories and Writing Pedagogies. Indonesian Journal of English Language Teaching 4(2), 91-110.

Inoue, M. (1997). Japanese students writing skills and its relationship to their academic lives in the United States. PhD dissertation. University of California, Santa Barbara. 
Ivanič, R. (1994). I is for interpersonal: Discoursal construction of writer identities and the teaching of writing. Linguistics and Education 6(1), 3-15.

Ivanič, R. (1998). Writing and Identity: The Discoursal Construction of Identity in Academic Writing. Amsterdam, The Netherlands: Benjamins.

Ivanič, R. (2004). Discourses of writing and learning to write. Language and Education, 18(3), 220-245.

Ivanič, R. \& Camps, D. (2001). I am how I sound: Voice as self-representation in L2 writing. Journal of Second Language Writing, 10(1-2), 3-33.

Jaffe, D. J., \& Miller, E.M. (1994). Problematizing meaning. In J.F. Gubrium \& A. Sankar (Eds.), Qualitative methods in aging research (pp.51-54). Thousand Oaks, CA: Sage.

Janopoulos, M. (1992). University of NS and NNS writing errors: A comparison. Journal of Second Language Writing, 1(2), 109-121.

Johns, A.M. (2011). The future of genre in L2 writing: Fundamental, but contested, instructional decisions. Journal of Second Language Writing, 20(1), 56-68.

Kachru, B. (1992). World Englishes: Approaches, issues, and resources. Language Teaching, 25, 1-14.

Kajiki, R. (1996). Inventory of University Entrance Examinations (Public Universities). Tokyo, Japan: Obunsha.

Kamimura, T. (1996). Composing in Japanese as a first language and English as a foreign language: A study of narrative writing. RELC Journal, 27, 47-69.

Kamimura, T. (2000). Integration of process and product orientations in EFL writing instruction. RELC Journal, 31(2), 1-28.

Kamimura, T. (2006). Effects of Peer Feedback on EFL Student Writers at Different Levels of English Proficiency: A Japanese Context. TESL Canada Journal, 23(2), 12-39.

Kamimura, T. \& Oi, K. (1997). Contrastive rhetoric in letter writing: The interaction of linguistic proficiency and cultural awareness. JALT Journal, 19, 58-76.

Kamimura, T. \& Oi, K. (1998). Argumentative strategies in American and Japanese English. World Englishes, 17(3), 307-323.

Kamimura, T. \& Oi, K. (2001). The effects of different points of view on the story production of Japanese EFL students. Foreign Language Annals, 34, 118-130.

Kamimura, T. \& Oi, K. (2006). A Developmental Perspective on Academic Writing 
Instruction for Japanese EFL Students. The Journal of Asia TEFL, 3(1), 97-129.

Kaplan, R.B. (1966). Cultural thought patterns in inter-cultural education. Language Learning, 16, 1-20.

Kaplan, R.B. (1987). Cultural thought patterns revisited. In U. Connor \& R.B. Kaplan (Eds.), Writing Across Languages: Analysis of L2 text (pp.9-21). Reading, MA: Addison-Wesley.

Kaufman, S.R. (1994). In-depth Interviewing. In J.F. Gubrium \& A. Sankar (Eds.), Qualitative methods in aging research (pp.123-136). Thousand Oaks, CA: Sage.

Keh, C.L. (1990). Feedback in the writing process: a model and methods for implementation. ELT Journal, 44(4), 294-304.

Kent, T. (2003). Post-Process Theory: Beyond the Writing Process Paradigm. Carbondale, IL: Southern Illinois UP.

Kroll, B. (1990). What does time buy? ESL performance on home versus class compositions. In B. Kroll (Ed.), Second language writing: Research insights for the classroom (pp. 140-154). Cambridge, UK: Cambridge University Press.

Kubota, R. (1992). Contrastive rhetoric of Japanese and English: A critical approach. $\mathrm{PhD}$ dissertation. University of Toronto, Canada.

Kubota, R. (1997). A reevaluation of the uniqueness of Japanese written discourse. Written Communication, 14(4), 460-480.

Kubota, R. (1998). An investigation of L1-L2 transfer in writing among Japanese university students: Implications for contrastive rhetoric. Journal of Second Language Writing, 7, 69100.

Kubota, R. (1999). Japanese culture constructed by discourses: Implications for applied linguistics research and ELT. TESOL Quarterly, 33(1), 9-35.

Kubota, R. (2002). Impact of Globalization on Language Teaching in Japan. In D. Block \& D. Cameron (Eds.), Globalization and language teaching (pp. 13-28). London, UK: Routledge.

Kubota, R. (2003). New approaches to gender, class, and race in second language writing. Journal of Second Language Writing, 12(1), 31-47.

Lantolf, J.P. \& Thorne, S.L. (2006). Sociocultural Theory and the Genesis of Second Language Development. Oxford, UK: Oxford University Press.

LeCompte, M.D.\& Preissle, J.(1993). Ethnography and Qualitative Design in Educational Research, (2nd ed.). New York: Academic Press. 
Lee-Cunin, M. (2005). The Japanese Student Perspective on Universities. In J.S. Eades, R. Goodman and Y. Hada (Eds.), The 'Big Bang' in Japanese Higher Education: The 2004 Reforms and the Dynamics of Change (pp.136-164). Melbourne, Australia: Trans Pacific Press.

Leki, I. (2000). Writing, Literacy, and Applied Linguistics. Annual Review of Applied Linguistics 20, 99-115.

Leki, I. \& Carson, J.G. (1994). Students' perceptions of EAP writing instruction and writing needs across the disciplines. TESOL Quarterly, 28(1), 81-101.

Leki, I., Cumming, A. \& Silva, T. (2008). A Synthesis of Research on Second Language Writing in English. New York: Routledge.

Liebman, J.D. (1992). Toward a new contrastive rhetoric: Differences between Arabic and Japanese rhetorical instruction. Journal of Second Language Writing, 1(2), 141-165.

Lincoln, Y.S. \& Guba, E.G. (1985). Naturalistic inquiry. Beverly Hills, CA: Sage.

Llewellyn, G., Sullivan, G. \& Minichiello, V. (1999). Sampling in qualitative research. In V. Minichiello, G. Sullivan , K. Greenwood, \& R. Axford, (Eds.), Handbook of Research Methods in Health (pp.73-199). Melbourne, Australia: Addison, Wesley, Longman.

LoCastro, V. (1996). English language education in Japan. In H. Coleman (Ed.) Society and the Language Classroom (pp. 40-58). Cambridge, UK: Cambridge University Press.

LoCastro, V. (2000). Evidence of accommodation to L2 pragmatic norms in peer review tasks by Japanese learners of English. JALT Journal, 22(2), 245-270.

Loveday, L.J. (1996). Language contact in Japan: A socio-linguistic history. New York: Oxford University Press.

Luppescu, S. \& Day, R.R. (1990). Examining attitude in teachers and students: the need to validate questionnaire data. Second Language Research, 6(2), 125-134.

Mack-Cozzo, J.B. (2002). If you think we have problems... Japan's inferior university system. American Enterprise, 13(6), 46-47.

Mann, L. (1980). Cross-cultural studies of small groups. In H. Triandis \& R.W. Brislin (Eds.), Handbook of cross-cultural psychology: Vol. 5. Social psychology (pp. 155-209). Boston, MA: Allyn \& Bacon.

Martin, J.R. (1997). Analysing genre: Functional parameters. In F. Christie and J.R. Martin (Eds.), Genre and Institutions: Social Processes in the Workplace and School (pp.3-39). London, UK: Cassell. 
Martin, J.R. (2000). Beyond Exchange: APPRAISAL Systems in English. In S. Hunston \& G. Thompson (Eds.). Evaluation in Text, Oxford, UK: Oxford University Press.

Martin, J.R. \& Rose, D. (2003). Working with discourse: meaning beyond the clause. London, UK: Continuum.

Martin, J.R. \& White, P.R.R. (2005). The Language of Evaluation: Appraisal in English. New York: Palgrave Macmillan.

Masao, K. (1976). The Japanese Language and Intercultural Communication. In K.

Tsutomu (Ed.), The Silent Power: Japan's Identity and World Role (pp. 51-73). Tokyo, Japan: Simul.

Matsubara, Y. (2001). Communicative Writing: Teaching Japanese High School Students to Use Writing to Communicate in English, 35 pages, Retrieved from:

http://www.eric.ed.gov/PDFS/ED452724.pdf

Matsuda, P.K. (1997). Contrastive rhetoric in context: A dynamic model of L2 writing. Journal of Second Language Writing, 6(1), 45-60.

Matsuda, P.K. (2001). Voice in Japanese written discourse Implications for second language writing. Journal of Second Language Writing, 10, 35-53.

Matsuda, P.K. (2003). Second language writing in the twentieth century: A situated historical perspective. In B. Kroll (Ed.), Exploring the dynamics of second language (pp.1534). Cambridge, UK: Cambridge University Press.

Matsuda, P.K. \& Tardy, C.M. (2007). Voice in academic writing: The rhetorical construction of author identity in blind manuscript review. English for Specific Purposes, 26, 235-249.

McCagg, P. (1996). If you can lead a horse to water, you don't have to make it drink: Some comments on reader and writer responsibilities. Multilingua, 15(3), 239-256.

McConnell, D.L. (1996). Education for global integration in Japan: a case study of the JET Program. Human organization, 55(4), 446-453.

McCrimmon, J.M. (1980). Writing With a Purpose. Boston, MA: Houghton Mifflin Company.

McFreely, N. (1999). A comparative study of the writing component of the language arts curricula in Japan and in California's secondary schools. PhD Dissertation. University of San Francisco.

McKinley, J. (2005). A Western Researcher in A Japanese University Writing Classroom: Limited or Advantaged? The Journal of Asia TEFL, 2(3), 139-146.

McVeigh, B.J. (2002). Japanese Higher Education as Myth. Armonk, NY: M.E. Sharpe. 
MEXT [Ministry of Education, Culture, Sports, Science, and Technology]. (2002). Developing a strategic plan to cultivate Japanese with English abilities: Plan to improve English and Japanese abilities. Retrieved from: http://www.mext.go.jp/english/mews/2002/07/ 020901.htm

Miles, M. \& Huberman, A.M. (1994). Qualitative Data Analysis: An expanded sourcebook ( $2^{\text {nd }}$ ed.). Thousand Oaks, CA: Sage.

Minichiello, V., Aroni, R., Timewell, E. \& Alexander, L. (1995). In-depth Interviewing: Principles, Techniques, Analysis ( $2^{\text {nd }}$ ed.). Melbourne, Australia: Longman Cheshire.

Mishler, E.G. (1986). Research Interviewing: Context and Narrative. Cambridge, MA: Harvard University Press.

Moore, G. \& Lamie, J. (1996). Translate or Communicate? English as a Foreign Language in Japanese High Schools. Commack, NY: Nova Science Publishers.

Morioka, K. (1963). Bunsho kosei ho: Bunsho no shindan to chiryo [Methods of text organization: Diagnoses and remedies of texts]. Tokyo, Japan: Shibundo.

Muncie, J. (2000). Using written teacher feedback in EFL composition classes. ELT Journal, 54(1), 47.

Murphy, J.W. \& Longino, C.F. Jr. (1992). What is the Justification for a Qualitative Approach to Ageing Studies? Ageing and Society, 12, 143-156.

Nakane, C. (1970). Japanese Society. Harmondsworth, UK: Penguin Books.

Neuman, W. L. (2003). Social research methods: Qualitative and quantitative approaches ( $^{\text {th }}$ ed.). Boston, MA: Allyn \&Bacon.

Noor, R. (2001). Contrastive rhetoric in expository prose: Approaches and achievements. Journal of Pragmatics, 33(2), 255-269.

Nunan, D. (2003). The impact of English as a global language on educational policies and practices in the Asia-Pacific region. TESOL Quarterly, 37(4), 589-613.

Oi, K., \& Kamimura, T. (1997). A pedagogical application of research in contrastive rhetoric. JACET Bulletin, 28, 65-82.

Olson, G.A. \& Dobrin, S.I. (Eds.). (1994). Composition theory for the post-modern classroom. Albany, NY: State University of New York Press.

Paltridge, B. (2001). Genre and the language learning classroom. Ann Arbor, MI: The University of Michigan Press. 
Paltridge, B. (2002a). Genre, text type and the EAP classroom. In A. Johns (Ed.), Genre in the Classroom: Multiple Perspectives (pp.73-90). Mahwah, NJ: Lawrence Erlbaum.

Patton, M.Q. (2002). Qualitative research and evaluation methods (3 ${ }^{\text {rd }}$ ed.). Thousand Oaks, CA: Sage.

Phelan, J. (2001). On Teaching Critical Arguments: A Matrix of Understanding. Pedagogy $1(3), 527-538$.

Poole, G.S. (2005). Reform of the university English language teaching curriculum in Japan: A case study. In J.S. Eades, R. Goodman \& Y. Hada (Eds.) The 'Big Bang' in Japanese Higher Education: The 2004 Reforms and the Dynamics of Change (pp.242-273). Melbourne, Australia: Trans Pacific Press.

Poole, G.S. (2010). The Japanese Professor: An Ethnography of a University Faculty. Rotterdam, The Netherlands: Sense Publishers.

Prior, P. (2001). Voices in text, mind, and society: Sociohistoric accounts of discourse acquisition and use. Journal of Second Language Writing, 10(1-2), 55-81.

Rabbini, R. (2003). The genre approach to writing assessed. Academic Exchange Quarterly 7(1), 126-132.

Raimes, A. (1983). Tradition and revolution in ESL teaching. TESOL Quarterly, 17, 535-552.

Ramanathan, V. \& Atkinson, D. (1999). Individualism, Academic Writing, and ESL Writers. Journal of Second Language Writing, 8(1), 45-75.

Rapley, D.J. (2008). Policy and Reality: The Teaching of Oral Communication By Japanese Teachers of English in Public High Schools in Kurashiki City, Japan. M.A. Dissertation, Massey University, New Zealand.

Ravelli, L.J. \& Ellis, R.A. (Eds.). (2005). Analysing Academic Writing: Contextualized Frameworks. London, UK: Continuum.

Reesor, M. (2003). Japanese Attitudes to English: Towards an Explanation of Poor Performance. Nagoya University of Commerce and Business: Journal of Language, Culture and Communication, 5(2), 57-65.

Reid, J. (2001). Writing. In D. Nunan (Ed.), The Cambridge guide to teaching English to speakers of other languages (pp.28-33). Cambridge, UK: Cambridge University Press.

Reid, K., Flowers, P. \& Larkin, M. (2005). Exploring Lived Experience. The Psychologist, 18(1), 20-23. 
Richards, J.C. \& Rodgers, T.S. (2001). Approaches and Methods in Language Teaching (2nd ed.). Cambridge, UK: Cambridge University Press.

Richards, K. (2003). Qualitative Inquiry in TESOL. Hampshire, UK: Palgrave Macmillan.

Rinnert, C. \& Kobayashi, H. (2001). Differing perceptions of EFL writing among readers in Japan. The Modern Language Journal, 85(ii), 189-209.

Robson, C. (2002). Real world research: A resource for social scientists and practitioner researchers (2 $2^{\text {nd }}$ ed.). Oxford, UK: Blackwell.

Russell, D.R. (1993). Vygotsky, Dewey, and externalism: Beyond the student/discipline dichotomy. Journal of Advanced Composition, 13, 173-198.

Sanders, T.J.M. \& Schilperoord, J. (2006). Text structure as a window on the cognition of writing: How text analysis provides insights in writing products and writing processes. In C. MacArthur, S. Graham \& J. Fitzgerald (Eds.), Handbook of Writing Research (pp.386-402), New York: Guilford Press.

Santos, T. (1992). Ideology in Composition: L1 and ESL. Journal of Second Language Writing, 1(1), 1-15.

Sasaki, M. (2005). Hypothesis generation and hypothesis testing: Two complementary studies of EFL writing processes. In P.K. Matsuda \& T. Silva (Eds.) Second Language Writing Research: Perspectives on the Process of Knowledge Construction (pp. 79-92). Mahwah, NJ: Lawrence Erlbaum.

Schilperoord, J. (1996). It's About Time: Temporal aspects of cognitive processes in text production. Amsterdam, The Netherlands: Rodopi.

Schneider, B. \& Andre, J. (2007). Developing Authority in Student Writing through Written Peer Critique in the Disciplines. The Writing Instructor. Retrieved from http://www.writinginstructor.com/schneider-andre

Scollon, R. (1991). Eight legs and an elbow: Stance and structure in Chinese English compositions. In Launching the literacy decade: Awareness into action. Proceedings of the Second North American Conference on Adult and Adolescent Literacy. Multiculturalism and citizenship (pp. l-14). Toronto, Canada: International Reading Association.

Seargeant, P. (2005). Globalisation and Reconfigured English in Japan. World Englishes, 24, 309-319.

Shinoda, Y. (April 29, 2006). English Writing Skills Essential For Students. The Asahi Shimbun.

Silva, T. (1993). Toward an understanding of the distinct nature of L2 writing: The ESL 
research and its implications. TESOL Quarterly, 27, 657-677.

Silva, T. (2005). On the Philosophical Bases of Inquiry in Second Language Writing: Metaphysics, Inquiry Paradigms, and the Intellectual Zeitgeist. In P.K. Matsuda \& T. Silva (Eds.) Second Language Writing Research: Perspectives on the Process of Knowledge Construction(pp.3-16). Mahwah, NJ: Lawrence Erlbaum.

Silverman, D. (2001). Interpreting qualitative data: Methods for analysing talk, text, and interaction. Thousand Oaks, CA: Sage.

Simon, S.D. (2010). The Principles of Constructivism, 9 pars., Retrieved from www.des.emory.edu/ mfp/502/502cons.PDF

Smith, R. (2010). Teaching English in Difficult Circumstances: A New Research Agenda [PowerPoint slides], retrieved from http://iatefl.britishcouncil.org/2010/sites/iatefl/files/session/documents/R._Smith__Teaching_in_Difficult_Circumstances_-_A_New_Research_Agenda_0.pdf

Sokol, M. (2005). Academic Identity Construction in E-discussion Lists: A Case Study. In G. Cortese \& A. Duszak (Eds.), Identity, Community, Discourse: English in Intercultural Settings (pp.321-344). Bern, Switzerland: Peter Lang.

Spack, R. (1997). The acquisition of academic literacy in a second language: a longitudinal case study. Written Communication, 14, 3-62.

Spindler, G \& Spindler, L. (1992). Cultural process and ethnography: An anthropological perspective. In M. LeCompte, W. Millroy, \& J. Preissle (Eds.), The Handbook of Qualitative Research in Education (pp.53-92). New York: Academic Press.

Stapleton, P. (1995). The Role of Confucianism in Japanese Education. The Language Teacher, 19(4), 13-16.

Stapleton, P. (2001). Assessing Critical Thinking in the Writing of Japanese University Students: Insights About Assumptions and Content Familiarity. Written Communication, 18(4), 506-548.

Stapleton, P. (2002a). Critical thinking in Japanese L2 writing: Re-thinking tired constructs. ELT Journal, 56(3), 250-257.

Stapleton, P. (2002b). Critiquing voice as a viable pedagogical tool in L2 writing: Returning the spotlight to ideas. Journal of Second Language Writing, 11, 177-190.

Stetsenko, A. \& Arievitch, I. (1997). Constructing and deconstructing the self: Comparing post-Vygotskian and discourse-based versions of social constructivism. Mind, Culture, and Activity, 4, 160-173. 
Storch, N. (2005). Collaborative writing: Product, process, and students' reflections. Journal of Second Language Writing, 14, 153-173.

Strauss, A. \& Corbin, J. (1990). Basics of qualitative research: Grounded theory procedures and techniques. Newbury Park, CA: Sage.

Swan, M. (1985a). A critical look at the communicative approach 1. ELT Journal, 39(1), 212.

Swan, M. (1985b). A critical look at the communicative approach 2. ELT Journal, 39(2), 7687.

Takemata, K. (1976). Genko Shippitsu Nyumon [An introduction to writing manuscripts]. Tokyo, Japan: Natsumesha.

Talmy, S. \& Richards, K. (2011). Theorizing Qualitative Research Interviews in Applied Linguistics. Applied Linguistics, 11(1), 1-5.

Taylor, I. \& Taylor, M. (1995). Writing and literacy in Chinese, Korean, and Japanese. Amsterdam: John Benjamins.

Trimbur, J. (1994). Taking the social turn: Teaching writing post-process. College Composition and Communication, 45, 108-118.

Tsui, A.B.M. (1996). Learning how to teach ESL writing. In D. Freeman \& J.C. Richards (Eds.), Teacher Learning in Language Teaching (pp.97-120). Cambridge, UK: Cambridge University Press.

Turner, J. (2011). Language in the Academy: Cultural Reflexivity and Intercultural Dynamics. Bristol, UK: Multiligual Matters.

Twine, N. (1991). Language and the Modern State: The Reform of Written Japanese. New York: Routledge.

Wijk, C. van \& T. Sanders (1999). Identifying writing strategies through text analysis. Written Communication, 1, 52-76.

Vianna, E. \& Stetsenko, A. (2006). Embracing History through Transforming It: Contrasting Piagetian versus Vygotskian (Activity) Theories of Learning and Development to Expand Constructivism within a Dialectical View of History. Theory \& Psychology, 16(1), 81-108.

Vygotsky, L.S. (1978). Mind in society. The development of higher psychological functions, M. Cole, V. John-Steiner, S. Scribner, \& E. Souberman (Eds.); M. Cole\& M. Lopez-Morillas (Trans.). Cambridge, MA: Harvard University Press.

Welsh, E. (2002). Dealing with Data: Using NVivo in the Qualitative Data Analysis Process. 
Forum Qualitative Sozialforschung / Forum: Qualitative Social Research, 3(2), Art. 26, 12 pars., Retrieved from http://www.qualitative-research.net/index.php/fqs/article/view/ $865 / 1880$

Wenger, E. (1998). Communities of Practice: Learning, meaning and identity. Cambridge, UK: Cambridge University Press.

Wertsch, J.V. (1993). Voices of the Mind: Sociocultural Approach to Mediated Action. Cambridge, MA: Harvard University Press.

Wertsch, J.V. (1998). Mind as action. New York: Oxford University Press.

West, M. (1960). Teaching English in Difficult Circumstances. London, UK: Longmans, Green.

White, R. \& Arndt, V. (1991). Process writing. Harlow, UK: Longman.

White, P.R.R. (2005). An introductory tour through appraisal theory. 28 pages, Retrieved from http://www.grammatics.com/appraisal/AppraisalOutline/Framed/ AppraisalOutline.htm

Yanabu, A. (1981). Nihongo o doo kaku ka [How do you write Japanese]. Tokyo, Japan: PHPsha.

Yasuda, S. (2005). Different Activities in the Same Task: An Activity Theory Approach to ESL Students' Writing Process. JALT Journal, 27(2), 139-168.

Yin, R.K. (1984). Case Study Research: Design and Methods (1 ${ }^{\text {st }}$ ed.). Newbury Park, CA: Sage.

Yin, R.K. (2002).Case Study Research: Design and Methods (3 $3^{\text {rd }}$ ed.). Thousand Oaks, CA: Sage.

Yin, R.K. (2009).Case Study Research: Design and Methods (4th ed.). Thousand Oaks, CA: Sage.

Yoshimura, T. (2001). Formal instruction of rhetorical patterns and the effectiveness of using L1 in argumentative writing in an EFL setting. Doctoral Dissertation, Temple University, Japan.

Zamel, V. (1982). Writing: The process of discovering meaning. TESOL Quarterly, 16(2), 195-209. 


\section{Appendices}

Appendix A

\section{Initial interview questions with students}

As the interviews were semi-structured, not all questions were asked to every participant. The initial interview questions were:

1. What is your experience with learning to write in English? Where? When?

2. What kind of writing assignments have you experienced so far? (journal, transcription, argument or analysis, report, research paper including literature review, others)

3. How was it (one of the writing assignments) organized?

4. Describe the process you go through in preparing for writing an essay.

5. What are your criteria for good English writing?

6. How has your writing skills development at this university helped you?

\section{Initial interview questions with teachers}

The following were my initial data collection questions with teachers. Similarly, not all questions were asked to every participant.

1. What is your experience with teaching English writing?

2. What is your opinion of the curriculum for writing skills development in this department?

3. Do you feel the emphasis on writing is sufficient? Why/why not?

4. What outcomes are students expected to obtain in your writing course? What particular skills do you feel need to be emphasised in writing?

\section{Final data collection questions with students}

The following questions were asked of the students in reference to the writing task selected for analysis. The questions follow the framework of a functional analysis as described by Ellis and Barkhuizen (2005):

Functional analysis (function-form, p.112) Questions used in follow-up interview 
1. Semantic function: What specific references are given? What non-specific references are given?

2. Does the grammar interfere with clarity at all?

Other linguistic characteristics (p.30)

3. Could you relate to this task? Do you understand why you were required to do it? Was it beneficial to your learning? Why? Why not?

4. Were there any difficulties affecting the outcome of this paper (assignment restrictions, etc.)? Did you have enough time?

5. Do you understand the features of this genre? Give examples.

6. Why did you choose to focus on these main ideas?

7. What about the references? How did you choose them?

Error Analysis (p.69) \& Frequency Analysis (p.93)

8. Can you identify/describe the errors?

9. Can you explain why they are errors?

\section{Appendix B}

\section{Participant information letter, statements and consent form}

The following information statements were distributed to each participant, and consent forms signed by each participant, approved by the Victoria University of Wellington's Human Ethics Committee. The following are included:

- Participant Letter for Teachers

- Participant Information Statement for Teachers

- Participant Information Statement for Students

- Participant Consent Form

The university name and researcher's contact details have been changed in order to maintain anonymity of the participants. 
Participant Letter for Teachers:

VICTORIA UNIVERSITY OF WELLINGTON

Te Whare Wananga o te Upoko o te Ika a Maui

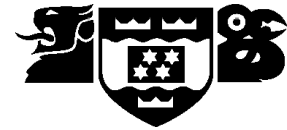

PO Box 600 - Wellington, NEW ZEALAND

Faculty of Humanities and Social Sciences

School of Linguistics and Applied Language Studies

A Study of University Students in Japan:

Learning and Application of Academic English Writing

April 2007

Dear Professor,

You are kindly requested to participate in a study into how university students in Japan learn and apply academic English writing skills. Through this study it is hoped students will become more aware of approaches they can use to become better writers of academic English.

The study is being conducted by Jim McKinley and will form the basis for the degree of PhD (Applied Linguistics) at Victoria University of Wellington under the supervision of Dr John Macalister. Ethics approval for this study has been granted by Victoria University's Human Ethics Committee.

Please see the attached Participant Information Statement for details about the study. Upon agreement to participate in the study, please read and sign the attached Participant Consent Form and return it to Jim McKinley.

Kind regards,

Jim McKinley

2 attachments 
VICTORIA UNIVERSITY OF WELLINGTON

Te Whare Wananga o te Upoko o te Ika a Maui

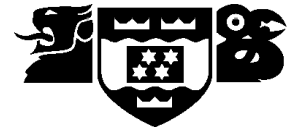

PO Box 600 - Wellington, NEW ZEALAND

Faculty of Humanities and Social Sciences

School of Linguistics and Applied Language Studies

\section{PARTICIPANT INFORMATION STATEMENT (Teacher)}

\section{Research Project}

Title: A Study of University Students in Japan: Learning and Application of Academic English Writing

April 2007

\section{(1) What does the study involve?}

The study will require your permission to allow Jim McKinley to observe and record your English composition classes once a month for one year. It also involves you taking part in monthly tape-recorded interviews conducted after your English composition classes (when you are available) in your office or Jim McKinley's office (2-918), whichever is more convenient for you, at [Midori University] over a period of one year. In these short interviews you will be asked about your approaches to teaching English composition: objectives, goals and experiences, and your attitude towards teaching English composition. You will also be asked to elaborate on your teaching methods for your composition class and strategies you use to develop students' English writing skills.

\section{(2) How much time will the study take?}

The study is expected to take up 30 minutes of your time for the interview each month (8 months in total).

\section{(3) Can I withdraw from the study?}

Being in this study is completely voluntary - you are not under any obligation to consent. Even after giving consent, you can withdraw from the project at any time. Data collected 
related to you will not be used in the study and will be destroyed immediately after withdrawal.

\section{(4) Will anyone else know the results?}

All aspects of the study, including results, will be strictly confidential and only the researcher will have access to information on participants. A report of the study may be submitted for publication, but individual participants will not be identifiable in such a report. In addition, participants may, at any time, choose to have any recordings of themselves deleted that they do not want used in the study.

\section{(5) Will the study benefit me?}

It is hoped that through the study, teachers will become more aware of their approaches to teaching English writing skills.

\section{(6) Can I tell other people about the study?}

Yes. There are no concealed motives to this study.

\section{(7) What if I require further information?}

When you have read this information, Jim McKinley will discuss it with you further and answer any questions you may have. If you would like to know more at any stage, please feel free to contact Jim McKinley, lecturer in the English Department at [Midori University] at mckinley@midori.ac.jp or 03 xxxx xxxx.

\section{(8) What if I have a complaint or concerns?}

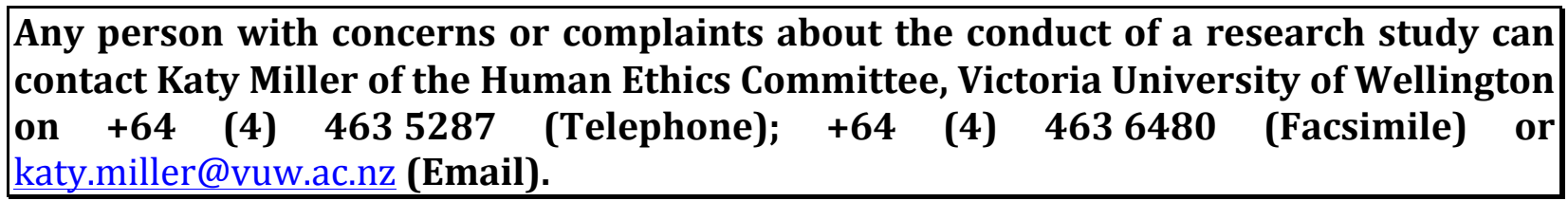

This information sheet is for you to keep. 
VICTORIA UNIVERSITY OF WELLINGTON

Te Whare Wananga o te Upoko o te Ika a Maui

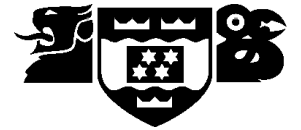

PO Box 600 - Wellington, NEW ZEALAND

Faculty of Humanities and Social Sciences

School of Linguistics and Applied Language Studies

\section{PARTICIPANT INFORMATION STATEMENT (Student)}

\section{Research Project}

Title: A Study of University Students in Japan: Learning and Application of Academic English Writing

April 2007

Dear Student,

You are invited to participate in a study into how university students in Japan learn and apply academic English writing skills. Through this study it is hoped students will become more aware of approaches they can use to become better writers of academic English.

\section{(1) Who is carrying out the study?}

The study is being conducted by Jim McKinley and will form the basis for the degree of PhD (Applied Linguistics) at Victoria University of Wellington under the supervision of Dr John Macalister. Ethics approval for this study has been granted by Victoria University's Human Ethics Committee.

\section{(2) What does the study involve?}

The study will involve you recording your writing class time (by wearing a voice recorder) and taking part in monthly tape-recorded interviews conducted after your English composition classes (when you are available) in meeting room 2-915 at [Midori University] over a period of one year. In these interviews you will be asked about your English learning background and experiences and your attitude towards learning English composition. You will also be asked to elaborate on your study methods before, during, and after each composition class and strategies you use to develop your English writing 
VICTORIA UNIVERSITY OF WELLINGTON

Te Whare Wananga o te Upoko o te Ika a Maui

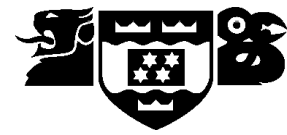

PO Box 600 - Wellington, NEW ZEALAND

Faculty of Humanities and Social Sciences

School of Linguistics and Applied Language Studies

\section{PARTICIPANT CONSENT FORM}

I give / do not give (please circle your response) my consent to participate in the study to be conducted from April 2007 by Jim McKinley, titled A Study of University Students in Japan: Learning and Application of Academic English Writing.

I understand that I may at any time withdraw from the study, and that my participation in this project will have no affect on my standing at [Midori University]. I also understand that my opinions and data will be kept strictly confidential in all reporting of findings.

Please indicate below whether you would like to receive a summary of the findings at the completion of the study.

$\circ$ Please send the summary of findings to the following e-mail address:

$\bigcirc$ Please send the summary of findings to the following postal address:

$\bigcirc \quad$ Please do not send a summary of the findings.

Signature

Date 


\section{Appendix C}

The essays from the four students selected as cases appear here as initially analyzed for possibilities for selfhood (autobiographical, authorial, and discoursal). All four texts showed multiple selves.

Case 1: Aya, complete essay analyzed for selves

Autobiographical (underlined) statements: 2

Authorial (in bold) statements: 50

Discoursal (in italics) statements: 29

Self: authorial-discoursal

It is still rather unusual event to see women who seem to be a Muslim and wear veil to cover their hair or face in Japan, even in a huge city like Tokyo is no exception. One hardly has chance to see women in a veil as walking through sub- centre cities. However as in European countries which holds a large number of immigrants from Muslim countries, the situation seems to be very different from that of Japan's.

Unlike Japan, issue of whether to let Muslim women to wear veil in pubic or not has been casing huge discussion within European countries. In 2003, President of France at that time, Jacques Chirac passed legislation which bans pupils of state schools to wear obvious religious signs, not only Islamic veil but also Christian cross to their schools. He claimed that secularism had been playing an important role to maintain harmony within French society. However, President of the France Council of the Muslim Faith, Dalil Boubakeur shows concern toward the legislation (BBC NEWS 18 December 2003.) According to BBC News, Rome, last updated 7th November 2006 by Christian Fraser, Italian Government are undergoing the process of making a new law to ban wearing veil that covers their faces hoping that it will prevent terrorism to take place. As in Britain, former Prime Minister, Tony Blaire called veiling as "mark of separation". (Alam 30 2006) Considering these facts of conflict and as Europe being a non-Muslim society, it seems that minority culture is going through a tough time and the issue of veil is still heatedly discussed among the society but no 
solution is seems to be found. This paper is aimed to have better understanding of the reasons why Muslim women veil and eager to find out what causes huge discussions that are based on cultural and religious differences focusing on Britain in particular by using a school girl, Shabina Begum's court case as an example.

It should be reminded before starting analyze of court case of Shabina Begum that the act of wearing veil had not always been seen as Islamic religious icon. According to the article, "Seeing Clearly" written by Carla Power and Rebecca Hall, in Newsweek, 27 November 2006 edition, Veiling activity already existed before Islam was founded by the Prophet Muhammad in seventh century in Arabian Peninsula. As seeing veil as religious object, it was what Judaism owned. As a cultural point of view, It was worn by upper-class Arab women in the Byzantine and Persian empires. For upper-class women of those regions, a veil was a symbol of the wealth. To wear a veil was a method to express their prosperity. As more women started to wear veil- the symbol of wealth- wearing veil became a method to distinguish upper-class women from lower-class women. It is after Islamic empire expanded that the social custom of wearing veil and sense of modesty in Islam got together.

There are several types of veil that Muslim women wear these days. Headscarf called "hijab" is most commonly worn piece of clothe that covers women's hair but shows their faces and bodies. As for Shabina Begum, it was jilbab - full length veil - she wished to wear. This paper is now going to analyze the court case of Shabina Begum from the following paragraphs.

In 2004, Shabina Begum who was a pupil of Denbigh High School in Luton, Bedfordshire went to High Court in order to have right to manifest her religious belief and wear jilbab - a traditional ankle length Muslim gown - to school. Miss Begum's fight began in 2002 when she told Denbigh High School, where 79\% of students are Muslims that she wishes to wear jilbab to school. School denied her right to wear what she intended to wear. The school told Miss Begum that they allowed pupils to wear traditional costume called Shalwar Kameez as school uniform. Miss Begum insisted on to wear jilbab out of her religious belief but then she was told that she can no longer attend her lessons and was 
sent home. She has not being attending school after being denied to wear jilbab and absence of Miss Begum continued for more that a year that she could not take her education properly during these time. (BBC NEWS) In 2004, after years of absence from school, she went to High Court to have her right to wear jilbab. However, her claim was not accepted by High Court. In 2005, though she was attending different school which allowed her to wear jilbab, she decided to go to Court of Appeal. Court of Appeal ruled in favor of Shabina Begum, saying that Debigh High School denied her right to practice her religious belief and called the school for instruction from Human Right Acts. Miss Begum called the ruling of House of Appeal "a victory for Muslims who wanted to preserve their identity and values" (Begum 02 March 2005) and she also stated that "It is amazing that in the so-called free world I have to fight to wear this attire." (Begum 02 March 2005) However, On March 22nd, 2006, Denbigh High School took the ruling of House of Appeal to the House of Lords. The Law Lords made announcement that they do not give her the right to wear what she wishes to wear. The judgment did not affect her directly as she had already left the school, her four years of fight in court ended with the denial.

Shabina Begum was fighting to get her right to wear jilbab but not her school uniform to school. However, as reading her statements and that of school's, it gives an impression that there is certain gap between her point of view and that of British's. The point Shabina Begum intended to make and what was important for her was different from those of her high school's. Having most of its pupils as Muslims, the school claimed and emphasized that they had given consideration to cultural and religious sensitivities when they decided their school uniform. They also said that they had consultation with the pupils, parents and local religious leaders. (BBC NEWS 22 March 2006) As reading news materials and protests that had been made by the high school from BBC NEWS online, it can be considered that the school took it for granted that people shared same understanding of characteristic of school uniform; something that the students must wear in proper way without an exception. In addition, it seems that the point school tried to emphasize was that they had given enough consideration toward the characteristics and circumstances of the religion when they made school uniform. Therefore, there is no chance for Shabina Begum to get justice to wear jilbab but not the uniform. When Miss 
Begum's case was taking place in High Court, her representative, Yvonne Spencer, according to BBC NEWS online, last updated 27th May 2004, said that Miss Begum's chance of passing the important examination was taken away by being excluded from the school just because she did not wear school uniform. Although High Court did not give ruling that favors Miss Begum, it is understandable that Yvonne Spencer to emphasized Shabina Begum's right to take part in education as a method to fight back and win a suit. However when she won the case under the ruling of Court of Appeal, she made several statements which was mainly about her right to manifest religion and she did not mention about her right to have education particularly.

It seems to be appropriate that the school was fighting on the ground of school uniform, its characteristic and fairness of when making it. It is also to the point that Yvonne Spencer was fighting back on the ground of education to win the case However, Shabina Begum was not on the same ground as the school was. She was not even on the same field as her representative. She did not make statements which were strongly connected to her right to have education as Yvonne Spencer was claiming on the trial or characteristic of school uniform and fairness of her school's uniform as her school was emphasizing. Her claim, as reading the statements she made that are written before, is more to do with the religion. Her claim was not just about school uniform versus jilbab, or whether jilbab should be accepted as school uniform. It was about how to live her life as good Muslim. Wearing jilbab is the way to show the God that she is living her life as good Muslim.

She had made several statements of how she feels about wearing jilbab and about the way of how she thinks of jilbab itself. The followings are the statements. "I feel it's an obligation on me to wear the jilbab as soon as I step outside my front door." (Begum 22 March 2006) "The jilbab is the clothing I feel was sent by the prophet." (Begum 22 March 2006) By reading these statements, it is quite obvious that she has very strong feeling toward wearing jilbab. As she states that to wear jilbab is "an obligation", wearing jilbab plays vital role in her daily life and also her mind, too. Therefore it cannot be separated from her life itself. In addition, it cannot be discussed only by the field of 
how characteristic of school uniform or how far a student can reflect her own wishes to the school uniform or education.

If one lives in secular society where religion does not play vital role in one's life, Shabina Begum's court case may sound extraordinary as she stepped out from her school and decided not to attend her lessons for more than a year for religious reason. Yet, she is not the only one who chose to wear veil in order to express what she believed in. The following is another example of women who chose to wear veil as method to express what she believed in.

Fareena Alam is a young British woman who has professional job, has received good education and was born in London but grew up in Singapore. She says in her article, "Beyond The Veil" written in Newsweek, unlike herself, none of her female family members chose to wear hijab, a headscarf. The sentences below are the reasons why she came to wear hijab.

"Driven by a strong sense of social justice and wanting to reconnect with my spirituality, I "found" Islam at university, where I was a campus activist. My decision to wear the head scarf, the hijab, at first had more to do with defining identity and a brash confidence about who I was and what values guided me. In time, it came to express my devotion as well." (Alam 30) As reading these sentences, it can be said that in the case of Fareena Alam, it was not her Islamic belief that made her wear veil at first, but she has something to share with Shabina Begum that they both decided to wear veil out of their intention to express what they believed in.

However, there is no absolute reason of why the women choose to wear veil that applies to every one of Muslim women. For those with ability of literacy, Qur'an and other reliable sources such as hadith -record of the Prophet's saying and doingcan be the reasons to wear veil. The interpretation of what the God tries to teach human being through Qur'an - written as a formation of poem - and other reliable sources is the responsibility of each individual, because there are no such people as clergyman but scholars in Islam, the scholars endeavor and interpret the Qur'an and other sources through their school of thoughts and there are several numbers of 
them. People seeks advice from scholars but since they have no clergyman or Popethe head of all the Christians- who decides what is a heretic thought, in Islam, strictly speaking, there is no one to decide what is a heretic thought or true belief. It is a responsibility of each individual to decide what to believe and not to believe. Therefore, it is each woman's responsibility and right to decide whether to wear veil or not.

As for Shabina Begum, she chose to wear veil out of her faith. However, interpretation of Qur'an and other sources are not only and the greatest reasons to wear veil. According to interview with professor lyama, whether they live in Muslim country or not, it is the Muslim society that makes or expect women to wear veil.

Fareena Alam says in her article about young British Muslims, herself included who are living outside of Muslim countries, that they are "the most globalized generation in European history connected to the countries of our parents or grandparents as well as to the broader spiritual community, umma, of world Islam." (Alam 32) According to her saying, young British Muslims are members of globalized society and also Muslim society. In addition, Muslim society is gaining its power according to Muslim journalist. He points out that for the Muslim in Britain today, the religion, Islam is becoming more influencing in daily lives and one's decision-making. (BBC NEWS 22 March 2006) As for Shabina Begum's court case, The Muslim Council of Britain (MCB), the UK's large Muslim community that profess themselves to be "UK's representative Muslim umbrella body" (MCB) made several announcement towards her court case through MCB press. They said that they see the decision which was made by High Court on 15 June 2004, as "worrying and objectionable." (MCB 15 June 2004) They also state that "Within this broad spectrum those that believe and choose to wear the jilbab and consider it to be part of their faith requirement of modest attire should be respected" (MCB 15 June 2004) However, they distinguish themselves from being a community to teach the interpretation and understanding of faith and this practice. As for Shabina Begum's victory under the ruling of House of Appeal, the head ofMCB, Iqubal Sacranie made declaration as following. "This is a very important ruling on the issue of personal freedom." (BBC NEWS 02 March 2005) 
The representative of British Muslim community did not make pronouncement that recommends women to wear veil as they state themselves to be "diverse community in terms of the interpretation and understanding of faith and its practice" (MCB 02 March 2005), but they did not show opposition toward Shabina Begum's action of going to court in order to get her right to wear jilbab. In fact, the Council considered her understanding of faith and its practice as respected.

Therefore, One consider under the circumstance as Islam greatly influences Muslim's daily life and decision-making that the fact of MCB making statement on Shabina Begum's court case and seeing her belief as respected proofs that Muslim community is indirectly expecting and welcoming women to wear veil so that they can express that they are living their life as good Muslim. It also, causes social impact on both Muslim community within Britain and that of none-Muslim's since they are affiliating 400 Muslim connected organizations and describes themselves as representative of British Muslim communities. MCB made Shabina Begum's court case a matter of their own rather than that of Miss Begum's by making those statements, and produced the environment where these kinds of acts will be respected.

Whether Shabina Begum's action was for her, out of social presser that Muslim society has or purely out of her will to please God, one considers that the interpretation of faith and its practice of her should not be insulted since she considers that religious belief plays vital role in creation of her identity and cannot be separated from her life. As for her case, it can not be solved as seeing the issue only on the ground of how far a pupil can reflect her wishes to school uniform. It is deeper than discussion of school uniform. It is strongly related to creation of her identity and her life itself.

However, Muslim side should be aware that their community co-exists with that of British's. In the same way that Muslim society has its own culture, that British society has its own culture, too. As for British society, it needs to understand how 
wearing veil or any other religious activities have deep connection with Muslim's life.

There seem to be no effective solutions yet to be found within Europe. However, as beginning of finding one of them, let it start with filling up the gaps between two cultures caused by misinterpretation of heart of the issue. It is time for both Muslim society and British society to stop seeing the issue only through their perspectives. It will take a huge amount of time and it may causes more complicated discussions to see things through different perspectives as religion seems to be at the core of Muslim's lives and as Europeans consider secularism is the greatest progress for them. However, as Fareena Alam described young British Muslims as "the most globalized generation within European history" in her article, it can be considered that there is a chance that the changes are going to be made. Again, considering what is written above, it is not straightforward problem that can be solved in one flick. However, it is the reality that there are Muslim societies within European societies. They both need to find the way to respect each other's cultures eventually and build a new society that is constructed of mixture of what the Europe had as their own culture and that of Muslim's.

Case 2: Ai, complete essay analyzed for selves

Autobiographical (underlined) statements: 0

Authorial (in bold) statements: 27

Discoursal (in italics) statements: 13

Self: authorial-discoursal

In which language do deaf students study better, sign language or Japanese? The answer to this question has changed as the education at schools for deaf and mute students progressed. In the $19^{\text {th }}$ Century, the main method of education for the deaf was the aural method which forced students to practice understanding and speaking Japanese with a hearing aid. At that time, sign language was thought to be an obstacle which deterred students from acquiring Japanese skills. However, this idea started to fade 
away in Japan from the 1980s as bilingual education, which allows students to learn sign language first and then learn the language of the majority population, has become increasingly popular like in the United States. In the article "New School to Use Signs as 1st Language" (the daily Yomiuri on June 21, 2007), Keiko Katayama reports that the nation's first accredited school for students with auditory difficulties is planned to be founded in Tokyo next spring. Thus, more schools where deaf students can learn in sign language should be founded in Japan because of mainly four reasons; it would enable them to enter the academic world, offer them an opportunity to feel proud of being deaf and treated more equally in education. Besides, it would give deaf students the chance to have jobs in the future, and eventually lead to an improvement in public welfare for the hearing challenged as understanding grows in society.

. Sign Language Enables the Deaf to Acquire Academic Abilities

First, deaf students could learn what they are interested in as hearing students can with less difficulties and frustration if they could use sign language while studying. In the article "The Debate Over Deaf Education", Burton Bollag explains how important sign language is for the deaf to learn by introducing the example of a deaf person who has succeeded in academics. The student's name is Daniel S. Koo and he is deaf by nature. He started to go to a public school where he was required to speak and listen to Japanese with a hearing aid, which made him frustrated because he could hardly understand what his teacher were saying. In the result, he felt behind other hearing students academically. However, after he moved to a school where he could use hand movements, that is, sign, his academic success story started. He entered the University of Maryland at College Park keeping up with his classes with the help of an interpret and he later went on to Gallaudet University for graduate studies, where all the classes he took were conducted in American Sign Language. Finally, today he is at Georgetown University Medical Center doing a postdoc in neurolinguistics. In addition, using sign language allows deaf students to feel free to talk in class. According to the book "Tyokakushogeisha no nihongoshido niokeru shuwa no shiyo ni kansuru kenkyu (The research about use of sign language in teaching Japanese to people with hearing difficulties)" Chonan Hirohito, a professor at Tsukuba Technology University, states that 
deaf students use more adjective and adverbs when they use sign language than in cases of written or spoken Japanese based on his experiment concerning 36 deaf high school students. This means that they feel less reluctance to speak in sign language and can express more details and richer content and thus learn higher-level content in sign language. From this research it can be said that sign language is indispensable as a deaf person's mother tongue and if it were not for sign language, the deaf would not have any language that they could use freely. For these reasons, deaf students should learn in sign language from elementary school so that they are ready to study academic subjects later.

\section{. Sign Language Gives Deaf Students Confidence}

The second strong point of using sign language at schools is that deaf students can feel proud of being deaf. Through the acquisition of sign language, the language they can use at their command, they would form their identities as a deaf individual. In the previously mentioned article "The Debate over Deaf Education," Burton cites a comment made by Mr. Koo, a deaf student who succeeded in academics. Mr. Koo explains that "ASL (American Sign Language) exposes children to the world's knowledge and it incorporates self-esteem and aspects of deaf culture." Deaf children can learn about what the deaf community is or what it means to be deaf through sign language. In addition, in the book "Nihon shuwa to robunka Uapanese sign language and the deaf culture)," Kimura Harumi states that deaf students who go to Ryunoko Schooli, which proceeds bilingual and bicultural education ${ }^{i i}$ for deaf in Japanese sign language, have confidence that they can sign and are able to state their opinions and listen to others' opinions whether their parents are deaf or not.(Kimura, 264) Deaf students become able to communicate with others without feeling fear by using sign language, even with people who are not hearing impaired if there is an interpreter. Thus, acquisition of sign language is considerably important for deaf persons to find their identities and self-esteem.

. Chance to have jobs 
Thirdly, deaf students can have more chances to secure an occupation in the future by making the most of there ability of signing improved in their school days. According to the resolution of the 11th World Congress of the World Federation of the Deafii held in Tokyo, if students can learn each subject in sign language, they improve their abilities in various subjects, which eventually gives them more opportunities to select their job. Resulting from the spread of such ideas in Japan, an increasing number of deaf people have occupations and play a great part in making the society better. For instance, Ippuku, who is a deaf, entertains people as a Rakugokaiv according to the article by DINF v"Traditional Japanese Comedy Performed in Sign Language." Sign Language Rakugo came to draw much attention since the 1980s, a time when bilingual education for deaf and mute students became popular as mentioned in the Introduction. Thus, the experiences of learning sign language itself and other subjects in sign language are significant for the deaf to increase their job opportunities in their future.

. Public facilities will be improved

Besides, public facilities will be improved as knowledge about deaf culture become more accessible as a result of the increasing number of deaf people who are active in the society. In the book "Chokaku gengo shogaisha to communication (People with linguistic and hearing difficulties and communication)," Ichibangase Yasuko states that for the improvement of the environment for the deaf it is necessary that people who are not hearing impaired understand about deaf culture and sign language to reduce barriers between them and deaf people. For example, New York has already began to improve public facilities for the deaf by approving agreements between consumer and state officials to offer 38 theaters with subtitles or narration in addition to the dozen theaters that now have such system(USA Today). Also, in Japan, Yahata General Hospital started to employ a sign language interpreter once a week on October.3rd (Sign Language Interpreter Was Placed). Thus, Japan should promote more familiarity between deaf people and others in society by drawing the attention of those who are not hearing impaired to the deaf community which would have the affect of increasing the number of schools for the deaf. 
. Lack of teachers

It is true that a large number of teachers is needed to establish many schools for deaf students. The fact that there are two kinds of sign language in Japan makes the problem even more difficult. Japanese sign language is what deaf people usually use and Sign Japanese was created by a person with no hearing challenges for Japanese and actually for deaf children, who do not understand Japanese grammar, the latter is difficult to comprehend. Therefore, teachers who can sign using Japanese sign language are needed though only deaf persons can sign using it. However, if the number of schools for the deaf and programs for training deaf teachers increases, those who want to teach using their ability of signing could can make the situation better. This would give deaf people opportunities to have more jobs as teachers.

\section{. Acquisition of Japanese}

Moreover, some people might say that if deaf children use sign language at school, they cannot acquire Japanese language skills though they are Japanese. According to the article"Kowa kyoiku wa nihongo kyoiku"(Aural teaching is Japanese teaching)by an Internet school for the deaf, deaf children who learn sign language early in their life cannot acquire Japanese easily because for them Japanese is a foreign language. However, they do not have to practice using Japanese if they have already had their own language as Japanese do not have to learn another foreign language like English. In addition, through bilingual education they can learn written Japanese and have almost the same ability in writing and reading Japanese as people who are not hearing impaired.

\section{. Conclusion}

Thus, Japan should have more schools for deaf students where they can learn sign language because they could catch up to regular students and learn academic subjects if they can use sign language while studying. Additionally, they would have more of a reason to feel proud of being deaf if they could communicate with others using sign language and the opportunities for deaf people to have a job would increase because they can find and improve their ability trying what they are 
interested in. Moreover, if there are a lot of deaf people who live actively in the society, public welfare would be made more convenient for deaf people. To increase the number of schools for deaf students from now on, more and more opportunities for people to know about the deaf community and the importance of sign language for deaf people.

$<$ End Notes $>$

${ }^{i}$ Ryunoko School was founded by NPO in Shinagawa Ward, Tokyo.

ii This is the slogan of Ryunoko School. Bilingual means Japanese sign language and written Japanes and bicultural means the deaf culture and the hearing culture.

iii The World Federation of the Deaf (WFD) is an international, non-governmental, central organization, whose members are from 123 countries.

iv Comic storytellers in Japan

v Disability INFormation Resources

Case 3: Rika, complete essay analyzed for selves

Autobiographical (underlined) statements: 0

Authorial (in bold) statements: 34

Discoursal (in italics) statements: 12

Self: authorial-discoursal

Tens of millions of animals are killed by animal testing every year (Feder). Animals have been used for disease research from long ago, and many still think animal testing is the best way to check if medicine is safe or not. This is because people only think about their own profits and do not think about animals' rights. Today, the number of people who are against animal testing is increasing and they say that there is no need for animal testing anymore. Using animals for disease research should be stopped because it is not the most reliable method for treating human health problems, too much tax money is used for the experiments and it is inhumane to use animals just for the profit of humans. 
To start with, animal testing is not the most reliable method for treating human health problems. Many types of medicine proven to be safe by animal testing had side effects on humans. Even though tested medicine may be safe on animals, it does not always mean it will be safe for humans too. There is no way to prove that the medicine will be safe for people if it is not checked on humans first. It is said that as much as twenty five percent of the medicines which was checked by animal testing could not show side effects which subsequently proved serious enough to prevent those medicines to be sold (Feder). This means it is very dangerous to use animal testing to prove whether or not medicine will be safe because serious side effects may show on humans even though it was not shown on animals. Also, incorrect data from animal testing leads to incorrect knowledge for treatment. If people continue to rely on data from animals, there will be a risk of more people suffering from side effects. The number of people who want to receive treatment without using animal testing is increasing, so it will not be long before animal testing becomes obsolete.

Secondly, too much tax money is used for animal experiments. Although a huge amount money was used to research cancer, nearly three million people were killed by malignant neoplasm (1), and the number of patients dying is still increasing for fifty years (ANIMAL RIGHTS CENTER). Animal testing costs a lot of money. Before medicine is tested on animals, animals have to be taken care of until that time. The money is used for food, maintaining the facilities for animals to live. If people knew how their tax money was being used for animal testing despite no reliable results from research, most people would condemn the use of their tax money for animal testing. Instead, tax money currently used for animals testing should be used for environmental conservation and pollution preservation.

Money for environmental conservation and pollution preservation is more needed than animal testing. Akio Morishima head of the Institute of Global Environmental Studies at Sophia University "Without doubt, the most important problem in the next century, both for Japan and for the world, will be global warming." (Corliss). This means that environmental problems are becoming more and more serious, so it is better to use money to prevent those problems rather than use money to take unreliable data 
from animal testings. Improvement of environmental circumstances and the standard of living is just as important as the development of medical care. For example, even if people are saved from dying as a result of medical care, their continued lives are not guranteed if the standard of living are poor. From this perspective, it is much more efficient to use money for improving people's standard of living rather than kill innocent animals in vain.

Lastly, it is inhumane to use animals just for the profit of humans. It is possible for animals to be used for research because they cannot speak and are weaker than humans. Not being able to speak, however, does not mean they do not have feelings. Although animals cannot speak human language, it is not difficult to imagine that they will suffer when medicine does not work. Also, animals are forced to be ill even though they are healthy, and some of them are born solely to be used for research. Animals born for the sole purpose of research could not possibly imagine that they were born to be killed. Fumie Hattori association president of JAVA said, "People don't know about the issues. Because they don't know they can be silent about them. When they do learn what's going on there's often a good response," (Bayer). Still, many people are only thinking about themselves and not thinking about animals killed by animal testing. Animals have the right to live too, and animal testing is an infringement on their rights.

Although there are many negative points about animal testing, there are still many people who say that animal testing should be continued. They argue that it would not be possible to make medicine to cure diseases if it was not for animal testing, and that most people who received the Nobel Prize for Medicine carried out such experiments (Pro-Test standing up for science). However, 'HUMAN skin, eyes, the lining of the throat - snippets of these and other tissues are now routinely grown in test tubes from donated human cells. The goal is not to patch up ailing people but to use the human tissues in place of mice, dogs or other lab animals for testing new drugs, cosmetics and other products." (Feder). There are alternatives to animal testings today, which do not cost as much as animal testing and are effective and humane (JAVA Doubutsu jikken haishi wo motomeru kai). In consideration of effective alternatives, there is no need to torture animals during disease research, 
and people can receive the Nobel Prize without sacrificing them. Improvement of technology allows alternatives to be used instead of animals, so animals should be freed and people should think about how to live in the world peacefully together with animals.

In conclusion, improvement of technology enabled companies to test medicines without animal testing and use alternatives such as human skins instead, and many people are becoming aware that it is difficult to rely on animal testing to treat human health problems, the amount of tax money used for the experiment is too much and it is no thinking about animals rights, so it is more likely to say that there is no need for animal testing any more. If more people posses the knowledge of how many animals are killed and how they are treated during animal testing, more people would fight for the improvement of animals' rights. Also, if people can dismiss their preconceptions about medicine which passed animal testing as being safe, more people will take notice of the effective alternatives. It is not just humans who are living in the world. It is time for humans to take action and fight for peace, both for humans themselves and for animals.

Case 4: Yui, complete essay analyzed for selves

Autobiographical (underlined) statements: 10

Authorial (in bold) statements: 16

Discoursal (in italics) statements: 0

Self: discoursal-autobiographical

In recent years, global warming has become a worldwide issue. Ice and glaciers are melting, the world's average temperature is rising, and irregular weather is causing many people to suffer and plants and animals to become extinct. The world seems like it's coming to an end. Although these problems from global warming are happening, there are some things we can all do to make this situation better. 
First of all, we should stop wasting energy. There are a many simple ways we can do this. First, always remember to turn off the lights when leaving the room. Second, do not let the water run while brushing your teeth or washing your face. Third, do not set the air conditioner to an excessive temperature. All these points stated seems like a little thing, but every degree, every minute lights are turned off, every millimeter of water saved, will change the world when it is done by many people. Just always keep in mind the word "mottainai" and you'll be somewhat contributing towards saving energy.

The second thing we can do to prevent global warming is to have minimal trash. Just say you do not need a plastic bag when going grocery shopping and say you do not need chopsticks when buying food at the convenience store. Carry your own shopping bag and your own chopsticks. Another thing is that companies making candies and snacks should consider overpackaging.-There is no need to wrap every candy, then wrap it again with a bigger bag, then put in a bag, then wrap the box, then put it in a box again. Once is enough for wrapping. If people consider overpackaging, it will lead to us having less trash.

Thirdly, to prevent global warming, we should rethink about our way of transporting. There is no need to use cars to go somewhere nearby. Walk to the nearest station and take buses and trains. Also, do not use one car per person. We should start carpooling with friends. This means fewer vehicles, which means less carbon dioxide in the air.

As a conclusion, there are many solutions for what we can do to prevent global warming. There are things we can start from today to save the world. All of these solutions listed have to be done by many people, not just one person. We should all reconsider about what we have to do to prevent global warming, which is necessary to save our own lives. 


\section{Appendix D}

The essays from the four students selected as cases appear three times each, once for each system (ATTITUDE, ENGaGement and GRADUATION) of the Appraisal Framework. The texts have not been edited, so misspellings and grammatical errors remain. The sections of the students' texts have been colored where indicators from the systems were identified, followed by the indicator in brackets. Additional notes about altered fonts are as follows:

ATTITUDE: Judgment is in italics, Appreciation is underlined, and Affect is in bold.

ENGAGEMENT: Modality is in italics, Attribution is underlined, Reality phases are in bold, Proclamations are in courier font, and Expectations are in comic sans font. There were no Counter-expectations in these cases.

GRADUATION: Force is in italics, and Focus is underlined.

\section{Case 1: Aya, complete essay marked for ATTITUDE}

It is still rather unusual event to see women [Judgment] who seem to be a Muslim and wear veil to cover their hair or face in Japan, even in a huge city like Tokyo is no exception. One hardly has chance to see women in a veil [Judgment] as walking through sub- centre cities. However as in European countries which holds a large number of immigrants from Muslim countries, the situation seems to be very different from that of Japan's.

Unlike Japan, issue of whether to let Muslim women to wear veil in pubic or not has been casing huge discussion [Appreciation] within European countries. In 2003, President of France at that time, Jacques Chirac passed legislation which bans pupils of state schools to wear obvious religious signs, not only Islamic veil but also Christian cross to their schools. He claimed that secularism had been playing an important role to maintain harmony within French society. However, President of the France Council of the Muslim Faith, Dalil Boubakeur shows concern toward the legislation (BBC NEWS 18 December 2003.) According to BBC News, Rome, last updated 7th November 2006 by Christian Fraser, Italian Government are undergoing the process of making a new law to ban wearing veil that covers their faces hoping that it will prevent terrorism to take place. As in Britain, former Prime Minister, Tony Blaire called veiling as "mark of separation". (Alam 30 2006) 
Considering these facts of conflict and as Europe being a non-Muslim society, it seems that minority culture is going through a tough time [Appreciation] and the issue of veil is still heatedly discussed among the society but no solution is seems to be found. This paper is aimed to have better understanding of the reasons why Muslim women veil and eager to find out what causes huge discussions [Affect: positive] that are based on cultural and religious differences focusing on Britain in particular by using a school girl, Shabina Begum's court case as an example.

It should be reminded before starting analyze of court case of Shabina Begum that the act of wearing veil had not always been seen as Islamic religious icon. According to the article, "Seeing Clearly" written by Carla Power and Rebecca Hall, in Newsweek, 27 November 2006 edition, Veiling activity already existed before Islam was founded by the Prophet Muhammad in seventh century in Arabian Peninsula. As seeing veil as religious object, it was what Judaism owned. As a cultural point of view, It was worn by upper-class Arab women in the Byzantine and Persian empires. For upper-class women of those regions, a veil was a symbol of the wealth. To wear a veil was a method to express their prosperity. As more women started to wear veil- the symbol of wealth- wearing veil became a method to distinguish upper-class women from lower-class women. It is after Islamic empire expanded that the social custom of wearing veil and sense of modesty in Islam got together.

There are several types of veil that Muslim women wear these days. Headscarf called "hijab" is most commonly worn piece of clothe that covers women's hair but shows their faces and bodies. As for Shabina Begum, it was jilbab - full length veil - she wished to wear. This paper is now going to analyze the court case of Shabina Begum from the following paragraphs.

In 2004, Shabina Begum who was a pupil of Denbigh High School in Luton, Bedfordshire went to High Court in order to have right to manifest her religious belief and wear jilbab - a traditional ankle length Muslim gown - to school. Miss Begum's fight began in 2002 when she told Denbigh High School, where 79\% of students are Muslims that she wishes to wear jilbab to school. School denied her right to wear what she intended to wear. 
The school told Miss Begum that they allowed pupils to wear traditional costume called Shalwar Kameez as school uniform. Miss Begum insisted on to wear jilbab out of her religious belief but then she was told that she can no longer attend her lessons and was sent home. She has not being attending school after being denied to wear jilbab and absence of Miss Begum continued for more that a year that she could not take her education properly during these time. (BBC NEWS) In 2004, after years of absence from school, she went to High Court to have her right to wear jilbab. However, her claim was not accepted by High Court. In 2005, though she was attending different school which allowed her to wear jilbab, she decided to go to Court of Appeal. Court of Appeal ruled in favor of Shabina Begum, saying that Debigh High School denied her right to practice her religious belief and called the school for instruction from Human Right Acts. Miss Begum called the ruling of House of Appeal "a victory for Muslims who wanted to preserve their identity and values" (Begum 02 March 2005) and she also stated that "It is amazing that in the so-called free world I have to fight to wear this attire." (Begum 02 March 2005) However, On March 22nd, 2006, Denbigh High School took the ruling of House of Appeal to the House of Lords. The Law Lords made announcement that they do not give her the right to wear what she wishes to wear. The judgment did not affect her directly as she had already left the school, her four years of fight in court ended with the denial.

Shabina Begum was fighting to get her right to wear jilbab but not her school uniform to school. However, as reading her statements and that of school's, it gives an impression that there is certain gap between her point of view and that of British's. The point Shabina Begum intended to make and what was important for her was different [Appreciation] from those of her high school's. Having most of its pupils as Muslims, the school claimed and emphasized that they had given consideration to cultural and religious sensitivities when they decided their school uniform. They also said that they had consultation with the pupils, parents and local religious leaders. (BBC NEWS 22 March 2006) As reading news materials and protests that had been made by the high school from BBC NEWS online, it can be considered that the school took it for granted [Judgment] that people shared same understanding of characteristic of school uniform; something that the students must wear in proper way without an exception. In addition, it seems that the point 
school tried to emphasize was that they had given enough consideration toward the characteristics and circumstances of the religion when they made school uniform. Therefore, there is no chance for Shabina Begum to get justice to wear jilbab but not the uniform. When Miss Begum's case was taking place in High Court, her representative, Yvonne Spencer, according to BBC NEWS online, last updated 27th May 2004, said that Miss Begum's chance of passing the important examination was taken away by being excluded from the school just because she did not wear school uniform. Although High Court did not give ruling that favors Miss Begum, it is understandable that Yvonne Spencer to emphasized Shabina Begum's right [Judgment] to take part in education as a method to fight back and win a suit. However when she won the case under the ruling of Court of Appeal, she made several statements which was mainly about her right to manifest religion and she did not mention about her right to have education particularly.

It seems to be appropriate that the school was fighting on the ground of school uniform [Judgment], its characteristic and fairness of when making it. It is also to the point that Yvonne Spencer was fighting back [Judgment] on the ground of education to win the case However, Shabina Begum was not on the same ground as the school was [Judgment]. She was not even on the same field as her representative [Judgment]. She did not make statements which were strongly connected to her right to have education as Yvonne Spencer was claiming on the trial or characteristic of school uniform and fairness of her school's uniform as her school was emphasizing. Her claim, as reading the statements she made that are written before, is more to do with the religion [Appreciation]. Her claim was not just about school uniform versus jilbab [Appreciation], or whether jilbab should be accepted as school uniform. It was about how to live her life as good Muslim. Wearing jilbab is the way to show the God that she is living her life as good Muslim.

She had made several statements of how she feels about wearing jilbab and about the way of how she thinks of jilbab itself. The followings are the statements. "I feel it's an obligation on me to wear the jilbab as soon as I step outside my front door." (Begum 22 March 2006) "The jilbab is the clothing I feel was sent by the prophet." (Begum 22 March 2006) By reading these statements, it is quite obvious that she has very strong feeling [Judgment] toward wearing jilbab. As she states that to wear jilbab is "an obligation", 
wearing jilbab plays vital role in her daily life and also her mind, too. Therefore it cannot be separated from her life itself. In addition, it cannot be discussed only by the field of how characteristic of school uniform or how far a student can reflect her own wishes to the school uniform or education.

If one lives in secular society where religion does not play vital role in one's life, Shabina Begum's court case may sound extraordinary [Appreciation] as she stepped out from her school and decided not to attend her lessons for more than a year for religious reason. Yet, she is not the only one who chose to wear veil in order to express what she believed in. The following is another example of women who chose to wear veil as method to express what she believed in.

Fareena Alam is a young British woman who has professional job, has received good education and was born in London but grew up in Singapore. She says in her article, "Beyond The Veil" written in Newsweek, unlike herself, none of her female family members chose to wear hijab, a headscarf. The sentences below are the reasons why she came to wear hijab.

"Driven by a strong sense of social justice and wanting to reconnect with my spirituality, I "found" Islam at university, where I was a campus activist. My decision to wear the head scarf, the hijab, at first had more to do with defining identity and a brash confidence about who I was and what values guided me. In time, it came to express my devotion as well." (Alam 30) As reading these sentences, it can be said that in the case of Fareena Alam, it was not her Islamic belief that made her wear veil at first, but she has something to share with Shabina Begum that they both decided to wear veil out of their intention to express what they believed in.

However, there is no absolute reason of why the women choose to wear veil that applies to every one of Muslim women. For those with ability of literacy, Qur'an and other reliable sources such as hadith -record of the Prophet's saying and doing- can be the reasons to wear veil. The interpretation of what the God tries to teach human being through Qur'an - written as a formation of poem - and other reliable sources [Appreciation] is the responsibility of each individual, because there are no such people as clergyman but 
scholars in Islam, the scholars endeavor and interpret the Qur'an and other sources through their school of thoughts and there are several numbers of them. People seeks advice from scholars but since they have no clergyman or Pope- the head of all the Christians- who decides what is a heretic thought, in Islam, strictly speaking, there is no one to decide what is a heretic thought or true belief. It is a responsibility of each individual to decide what to believe and not to believe. Therefore, it is each woman's responsibility and right to decide whether to wear veil or not.

As for Shabina Begum, she chose to wear veil out of her faith. However, interpretation of Qur'an and other sources are not only and the greatest reasons to wear veil. According to interview with professor lyama, whether they live in Muslim country or not, it is the Muslim society that makes or expect women to wear veil.

Fareena Alam says in her article about young British Muslims, herself included who are living outside of Muslim countries, that they are "the most globalized generation in European history connected to the countries of our parents or grandparents as well as to the broader spiritual community, umma, of world Islam." (Alam 32) According to her saying, young British Muslims are members of globalized society and also Muslim society. In addition, Muslim society is gaining its power according to Muslim journalist. He points out that for the Muslim in Britain today, the religion, Islam is becoming more influencing [Appreciation] in daily lives and one's decision-making. (BBC NEWS 22 March 2006) As for Shabina Begum's court case, The Muslim Council of Britain (MCB), the UK's large Muslim community that profess themselves to be "UK's representative Muslim umbrella body" (MCB) made several announcement towards her court case through MCB press. They said that they see the decision which was made by High Court on 15 June 2004, as "worrying and objectionable." (MCB 15 June 2004) They also state that "Within this broad spectrum those that believe and choose to wear the jilbab and consider it to be part of their faith requirement of modest attire should be respected" (MCB 15 June 2004) However, they distinguish themselves from being a community to teach the interpretation and understanding of faith and this practice. As for Shabina Begum's victory under the ruling of House of Appeal, the head ofMCB, Iqubal Sacranie made declaration as following. "This is a very important ruling on the issue of personal freedom." (BBC NEWS 02 March 2005) 
The representative of British Muslim community did not make pronouncement that recommends women to wear veil as they state themselves to be "diverse community in terms of the interpretation and understanding of faith and its practice" (MCB 02 March 2005), but they did not show opposition toward Shabina Begum's action of going to court in order to get her right to wear jilbab. In fact, the Council considered her understanding of faith and its practice as respected.

Therefore, One consider under the circumstance as Islam greatly influences Muslim's daily life and decision-making [Appreciation] that the fact of MCB making statement on Shabina Begum's court case and seeing her belief as respected proofs that Muslim community is indirectly expecting and welcoming women to wear veil [Judgment] so that they can express that they are living their life as good Muslim. It also causes social impact on both Muslim community within Britain and that of none-Muslim's since they are affiliating 400 Muslim connected organizations and describes themselves as representative of British Muslim communities. MCB made Shabina Begum's court case a matter of their own rather than that of Miss Begum's by making those statements, and produced the environment where these kinds of acts will be respected.

Whether Shabina Begum's action was for her, out of social presser that Muslim society has or purely out of her will to please God, one considers that the interpretation of faith and its practice of her should not be insulted [Judgment] since she considers that religious belief plays vital role in creation of her identity and cannot be separated from her life. As for her case, it can not be solved as seeing the issue only on the ground of how far a pupil can reflect her wishes to school uniform. It is deeper than discussion of school uniform [Appreciation]. It is strongly related to creation of her identity and her life itself [Appreciation].

However, Muslim side should be aware that their community co-exists with that of British's. In the same way that Muslim society has its own culture, that British society has its own culture, too. As for British society, it needs to understand how wearing veil or any other religious activities have deep connection with Muslim's life. 
There seem to be no effective solutions [Appreciation] yet to be found within Europe. However, as beginning of finding one of them, let it start with filling up the gaps between two cultures caused by misinterpretation of heart of the issue [Appreciation]. It is time for both Muslim society and British society to stop seeing the issue only through their perspectives. It will take a huge amount of time and it may causes more complicated discussions to see things through different perspectives as religion seems to be at the core of Muslim's lives and as Europeans consider secularism is the greatest progress for them. However, as Fareena Alam described young British Muslims as "the most globalized generation within European history" in her article, it can be considered that there is a chance that the changes are going to be made. Again, considering what is written above, it is not straightforward problem that can be solved in one flick [Appreciation]. However, it is the reality that there are Muslim societies within European societies. They both need to find the way to respect each other's cultures eventually and build a new society that is constructed of mixture of what the Europe had as their own culture and that of Muslim's.

\section{Case 1: Aya, complete essay marked for ENGAGEMENT}

It is still rather unusual event to see women who seem to be a Muslim and wear veil to cover their hair or face in Japan, even in a huge city like Tokyo is no exception. One hardly has chance to see women in a veil as walking through sub- centre cities. However as in European countries which holds a large number of immigrants from Muslim countries, the situation seems to be very different [Reality phase] from that of Japan's.

Unlike Japan, issue of whether to let Muslim women to wear veil in pubic or not has been casing huge discussion within European countries. In 2003, President of France at that time, Jacques Chirac [Attribution] passed legislation which bans pupils of state schools to wear obvious religious signs, not only Islamic veil but also Christian cross to their schools. He claimed that [Attribution] secularism had been playing an important role to maintain harmony within French society. However, President of the France Council of the Muslim Faith, Dalil Boubakeur shows concern [Attribution] toward the legislation (BBC NEWS 18 December 2003.) According to BBC News, Rome [Attribution], last updated 7th November 2006 by Christian Fraser, Italian Government are undergoing the process of making a new law to ban wearing veil that covers their faces hoping that it will prevent 
terrorism to take place. As in Britain, former Prime Minister, Tony Blaire [Attribution] called veiling as "mark of separation". (Alam 30 2006) Considering these facts of conflict and as Europe being a non-Muslim society, it seems that minority culture is going through a tough time [Reality phase] and the issue of veil is still heatedly discussed among the society but no solution is seems to be found [Reality phase]. This paper is aimed to have better understanding of the reasons why Muslim women veil and eager to find out what causes huge discussions that are based on cultural and religious differences focusing on Britain in particular by using a school girl, Shabina Begum's court case as an example.

It should be reminded [Modality: obligation] before starting analyze of court case of Shabina Begum that the act of wearing veil had not always been seen as Islamic religious icon. According to the article, "Seeing Clearly" [Attribution] written by Carla Power and Rebecca Hall, in Newsweek, 27 November 2006 edition, Veiling activity already existed before Islam was founded by the Prophet Muhammad in seventh century in Arabian Peninsula. As seeing veil as religious object, it was what Judaism owned. As a cultural point of view, It was worn by upper-class Arab women in the Byzantine and Persian empires. For upper-class women of those regions, a veil was a symbol of the wealth. To wear a veil was a method to express their prosperity. As more women started to wear veil- the symbol of wealth- wearing veil became a method to distinguish upper-class women from lower-class women. It is after Islamic empire expanded that the social custom of wearing veil and sense of modesty in Islam got together.

There are several types of veil that Muslim women wear these days. Headscarf called "hijab" is most commonly worn piece of clothe that covers women's hair but shows their faces and bodies. As for Shabina Begum, it was jilbab - full length veil - she wished to wear. This paper is now going to analyze the court case of Shabina Begum from the following paragraphs.

In 2004, Shabina Begum who was a pupil of Denbigh High School in Luton, Bedfordshire went to High Court in order to have right to manifest her religious belief and wear jilbab - a traditional ankle length Muslim gown - to school. Miss Begum's fight began 
in 2002 when she told Denbigh High School, where 79\% of students are Muslims that she wishes to wear jilbab to school. School denied her right to wear what she intended to wear. The school told Miss Begum that they allowed pupils to wear traditional costume called Shalwar Kameez as school uniform. Miss Begum insisted on to wear jilbab out of her religious belief but then she was told that she can no longer attend her lessons and was sent home. She has not being attending school after being denied to wear jilbab and absence of Miss Begum continued for more that a year that she could not take her education properly during these time. (BBC NEWS) In 2004, after years of absence from school, she went to High Court to have her right to wear jilbab. However, her claim was not accepted by High Court. In 2005, though she was attending different school which allowed her to wear jilbab, she decided to go to Court of Appeal. Court of Appeal ruled in favor of Shabina Begum, saying that [Attribution] Debigh High School denied her right to practice her religious belief and called the school for instruction from Human Right Acts. Miss Begum called the ruling [Attribution] of House of Appeal "a victory for Muslims who wanted to preserve their identity and values" (Begum 02 March 2005) and she also stated that [Attribution] "It is amazing that in the so-called free world I have to fight to wear this attire." (Begum 02 March 2005) However, On March 22nd, 2006, Denbigh High School took the ruling of House of Appeal to the House of Lords. The Law Lords made announcement that they do not give her the right to wear what she wishes to wear. The judgment did not affect her directly as she had already left the school, her four years of fight in court ended with the denial.

Shabina Begum was fighting to get her right to wear jilbab but not her school uniform to school. However, as reading her statements and that of school's, it gives an impression that there is certain gap [Reality phase] between her point of view and that of British's. The point Shabina Begum intended to make and what was important for her was different from those of her high school's. Having most of its pupils as Muslims, the school claimed [Attribution] and emphasized that they had given consideration to cultural and religious sensitivities when they decided their school uniform. They also said [Attribution] that they had consultation with the pupils, parents and local religious leaders. (BBC NEWS 22 March 2006) As reading news materials and protests that had been made 
by the high school from BBC NEWS online [Attribution], it can be considered that the school took it for granted [Modality: probability] that people shared same understanding of characteristic of school uniform; something that the students must wear in proper way without an exception. In addition, it seems that the point [Reality phase] school tried to emphasize was that they had given enough consideration toward the characteristics and circumstances of the religion when they made school uniform. Therefore, there is no chance for Shabina Begum to get justice [Proclamation] to wear jilbab but not the uniform. When Miss Begum's case was taking place in High Court, her representative, Yvonne Spencer, according to BBC NEWS online, last updated 27th May 2004, said [Attribution] that Miss Begum's chance of passing the important examination was taken away by being excluded from the school just because she did not wear school uniform. Although High Court did not give ruling that favors Miss Begum, it is understandable that Yvonne Spencer to emphasized Shabina Begum's right to take part in education as a method to fight back and win a suit. However when she won the case under the ruling of Court of Appeal, she made several statements which was mainly about her right to manifest religion and she did not mention about her right to have education particularly.

It seems to be appropriate [Reality phase] that the school was fighting on the ground of school uniform, its characteristic and fairness of when making it. It is also to the point that Yvonne Spencer was fighting back on the ground of education to win the case However, Shabina Begum was not on the same ground as the school was. She was not even on the same field as her representative. She did not make statements which were strongly connected to her right to have education as Yvonne Spencer was claiming on the trial or characteristic of school uniform and fairness of her school's uniform as her school was emphasizing. Her claim, as reading the statements she made that are written before, is more to do with the religion. Her claim was not just about school uniform versus jilbab, or whether jilbab should be accepted as school uniform. It was about how to live her life as good Muslim. Wearing jilbab is the way to show the God that she is living her life as good Muslim. 
She had made several statements of how she feels about wearing jilbab and about the way of how she thinks of jilbab itself. The followings are the statements. "I feel it's an obligation on me to wear the jilbab as soon as I step outside my front door." (Begum 22 March 2006) "The jilbab is the clothing I feel was sent by the prophet." (Begum 22 March 2006) By reading these statements [Attribution], it is quite obvious that she has very strong feeling toward wearing jilbab. As she states that [Attribution] to wear jilbab is "an obligation", wearing jilbab plays vital role in her daily life and also her mind, too. Therefore it cannot be separated from her life itself. In addition, it cannot be discussed only by the field of how characteristic of school uniform or how far a student can reflect her own wishes to the school uniform or education.

If one lives in secular society where religion does not play vital role in one's life, Shabina Begum's court case may sound extraordinary [Modality: probability] as she stepped out from her school and decided not to attend her lessons for more than a year for religious reason. Yet, she is not the only one who chose to wear veil in order to express what she believed in. The following is another example of women who chose to wear veil as method to express what she believed in.

Fareena Alam is a young British woman who has professional job, has received good education and was born in London but grew up in Singapore. She says in her article, "Beyond The Veil" [Attribution] written in Newsweek, unlike herself, none of her female family members chose to wear hijab, a headscarf. The sentences below are the reasons why she came to wear hijab.

"Driven by a strong sense of social justice and wanting to reconnect with my spirituality, I "found" Islam at university, where I was a campus activist. My decision to wear the head scarf, the hijab, at first had more to do with defining identity and a brash confidence about who I was and what values guided me. In time, it came to express my devotion as well." (Alam 30) As reading these sentence [Attribution], it can be said [Proclamation] that in the case of Fareena Alam, it was not her Islamic belief that made her wear veil at first, but she has something to share with Shabina Begum that they both decided to wear veil out of their intention to express what they believed in. 
However, there is no absolute reason [Proclamation] of why the women choose to wear veil that applies to every one of Muslim women. For those with ability of literacy, Qur'an and other reliable sources such as hadith -record of the Prophet's saying and doing- can be the reasons to wear veil. The interpretation of what the God tries to teach human being through Qur'an - written as a formation of poem - and other reliable sources is the responsibility of each individual, because there are no such people as clergyman but scholars in Islam, the scholars endeavor and interpret the Qur'an and other sources through their school of thoughts and there are several numbers of them. People seeks advice from scholars but since they have no clergyman or Pope- the head of all the Christians- who decides what is a heretic thought, in Islam, strictly speaking, there is no one to decide what is a heretic thought or true belief. It is a responsibility of each individual to decide what to believe and not to believe. Therefore, it is each woman's responsibility and right to decide whether to wear veil or not.

As for Shabina Begum, she chose to wear veil out of her faith. However, interpretation of Qur'an and other sources are not only and the greatest reasons to wear veil. According to interview with professor lyama [Attribution], whether they live in Muslim country or not, it is the Muslim society that makes or expect women to wear veil.

Fareena Alam says in her article [Attribution] about young British Muslims, herself included who are living outside of Muslim countries, that they are "the most globalized generation in European history connected to the countries of our parents or grandparents as well as to the broader spiritual community, umma, of world Islam." (Alam 32) According to her saying [Attribution], young British Muslims are members of globalized society and also Muslim society. In addition, Muslim society is gaining its power according to Muslim journalist. He points out that [Attribution] for the Muslim in Britain today, the religion, Islam is becoming more influencing in daily lives and one's decision-making. (BBC NEWS 22 March 2006) As for Shabina Begum's court case, The Muslim Council of Britain (MCB), the UK's large Muslim community that profess themselves to be "UK's representative Muslim umbrella body" (MCB) made several announcement towards her court case through MCB press. They said that [Attribution] they see the decision which was made by High Court on 15 June 2004, as "worrying and objectionable." (MCB 15 June 2004) They 
also state that [Attribution] "Within this broad spectrum those that believe and choose to wear the jilbab and consider it to be part of their faith requirement of modest attire should be respected" (MCB 15 June 2004) However, they distinguish themselves from being a community to teach the interpretation and understanding of faith and this practice. As for Shabina Begum's victory under the ruling of House of Appeal, the head of MCB, Iqubal Sacranie made declaration as following [Attribution]. "This is a very important ruling on the issue of personal freedom." (BBC NEWS 02 March 2005)

The representative of British Muslim community did not make pronouncement that recommends women to wear veil as they state themselves to be "diverse community in terms of the interpretation and understanding of faith and its practice" (MCB 02 March 2005), but they did not show opposition toward Shabina Begum's action of going to court in order to get her right to wear jilbab. In fact, the Council considered [Proclamation] her understanding of faith and its practice as respected.

Therefore, One consider under the circumstance as Islam greatly influences Muslim's daily life and decision-making that the fact of MCB making statement on Shabina Begum's court case and seeing her belief as respected proofs that Muslim community is indirectly expecting and welcoming women to wear veil so that they can express that they are living their life as good Muslim. It also causes social impact on both Muslim community within Britain and that of none-Muslim's since they are affiliating 400 Muslim connected organizations and describes themselves as representative of British Muslim communities. MCB made Shabina Begum's court case a matter of their own rather than that of Miss Begum's by making those statements, and produced the environment where these kinds of acts will be respected.

Whether Shabina Begum's action was for her, out of social presser that Muslim society has or purely out of her will to please God, one considers that the interpretation of faith and its practice of her should not be insulted [Modality: obligation] since she considers that religious belief plays vital role in creation of her identity and cannot be separated from her life. As for her case, it can not be solved as seeing the issue only on the ground of how 
far a pupil can reflect her wishes to school uniform. It is deeper than discussion of school uniform. It is strongly related to creation of her identity and her life itself.

However, Muslim side should be aware [Modality: obligation] that their community co-exists with that of British's. In the same way that Muslim society has its own culture, that British society has its own culture, too. As for British society, it needs to understand [Modality: obligation] how wearing veil or any other religious activities have deep connection with Muslim's life.

There seem to be no effective solutions [Reality phase] yet to be found within Europe. However, as beginning of finding one of them, let it start with filling up the gaps between two cultures caused by misinterpretation of heart of the issue. It is time for both Muslim society and British society to stop seeing the issue only through their perspectives [Proclamation]. It will take a huge amount of time [Expectation] and it may causes more complicated discussions [Modality: probability] to see things through different perspectives as religion seems to be at the core [Reality phase] of Muslim's lives and as Europeans consider secularism is the greatest progress for them. However, as Fareena Alam described young British Muslims [Attribution] as "the most globalized generation within European history" in her article, it can be considered that [Reality phase] there is a chance that the changes are going to be made [Expectation]. Again, considering what is written above, it is not straightforward problem that can be solved in one flick. However, it is the reality that there are Muslim societies within European societies. They both need to find the way to respect each other's cultures [Modality: obligation] eventually and build a new society that is constructed of mixture of what the Europe had as their own culture and that of Muslim's.

Case 1: Aya, complete essay marked for GRADUATION

It is still rather unusual event [Force] to see women who seem to be a Muslim and wear veil to cover their hair or face in Japan, even in a huge city like Tokyo [Force] is no exception. One hardly has chance [Force] to see women in a veil as walking through sub- 
centre cities. However as in European countries which holds a large number of immigrants from Muslim countries, the situation seems to be very different from that of Japan's.

Unlike Japan, issue of whether to let Muslim women to wear veil in pubic or not has been casing huge discussion [Force] within European countries. In 2003, President of France at that time, Jacques Chirac passed legislation which bans pupils of state schools to wear obvious religious signs, not only Islamic veil but also Christian cross to their schools. He claimed that secularism had been playing an important role to maintain harmony within French society. However, President of the France Council of the Muslim Faith, Dalil Boubakeur shows concern toward the legislation (BBC NEWS 18 December 2003.) According to BBC News, Rome, last updated 7th November 2006 by Christian Fraser, Italian Government are undergoing the process of making a new law to ban wearing veil that covers their faces hoping that it will prevent terrorism to take place. As in Britain, former Prime Minister, Tony Blaire called veiling as "mark of separation". (Alam 30 2006) Considering these facts of conflict and as Europe being a non-Muslim society, it seems that minority culture is going through a tough time and the issue of veil is still heatedly discussed [Focus] among the society but no solution is seems to be found. This paper is aimed to have better understanding of the reasons why Muslim women veil and eager to find out what causes huge discussions [Force] that are based on cultural and religious differences focusing on Britain in particular by using a school girl, Shabina Begum's court case as an example.

It should be reminded before starting analyze of court case of Shabina Begum that the act of wearing veil had not always been seen [Force] as Islamic religious icon. According to the article, "Seeing Clearly" written by Carla Power and Rebecca Hall, in Newsweek, 27 November 2006 edition, Veiling activity already existed before Islam was founded by the Prophet Muhammad in seventh century in Arabian Peninsula. As seeing veil as religious object, it was what Judaism owned. As a cultural point of view, It was worn by upper-class Arab women in the Byzantine and Persian empires. For upper-class women of those regions, a veil was a symbol of the wealth. To wear a veil was a method to express their prosperity. As more women started to wear veil- the symbol of wealth- wearing veil became a method to distinguish upper-class women from lower-class women. It is after 
Islamic empire expanded that the social custom of wearing veil and sense of modesty in Islam got together.

There are several types of veil that Muslim women wear these days. Headscarf called "hijab" is most commonly worn piece of clothe that covers women's hair but shows their faces and bodies. As for Shabina Begum, it was jilbab - full length veil - she wished to wear. This paper is now going to analyze the court case of Shabina Begum from the following paragraphs.

In 2004, Shabina Begum who was a pupil of Denbigh High School in Luton, Bedfordshire went to High Court in order to have right to manifest her religious belief and wear jilbab - a traditional ankle length Muslim gown - to school. Miss Begum's fight began in 2002 when she told Denbigh High School, where 79\% of students are Muslims that she wishes to wear jilbab to school. School denied her right to wear what she intended to wear. The school told Miss Begum that they allowed pupils to wear traditional costume called Shalwar Kameez as school uniform. Miss Begum insisted on to wear jilbab out of her religious belief but then she was told that she can no longer attend her lessons and was sent home. She has not being attending school after being denied to wear jilbab and absence of Miss Begum continued for more that a year that she could not take her education properly during these time. (BBC NEWS) In 2004, after years of absence from school, she went to High Court to have her right to wear jilbab. However, her claim was not accepted by High Court. In 2005, though she was attending different school which allowed her to wear jilbab, she decided to go to Court of Appeal. Court of Appeal ruled in favor of Shabina Begum, saying that Debigh High School denied her right to practice her religious belief and called the school for instruction from Human Right Acts. Miss Begum called the ruling of House of Appeal "a victory for Muslims who wanted to preserve their identity and values" (Begum 02 March 2005) and she also stated that "It is amazing that in the so-called free world I have to fight to wear this attire." (Begum 02 March 2005) However, On March 22nd, 2006, Denbigh High School took the ruling of House of Appeal to the House of Lords. The Law Lords made announcement that they do not give her the right to wear what she wishes to wear. The judgment did not affect her directly [Focus] as she had already left the school, her four years of fight in court ended with the denial. 
Shabina Begum was fighting to get her right to wear jilbab but not her school uniform to school. However, as reading her statements and that of school's, it gives an impression that there is certain gap between her point of view and that of British's. The point Shabina Begum intended to make and what was important for her was different from those of her high school's. Having most of its pupils as Muslims, the school claimed and emphasized that they had given consideration to cultural and religious sensitivities when they decided their school uniform. They also said that they had consultation with the pupils, parents and local religious leaders. (BBC NEWS 22 March 2006) As reading news materials and protests that had been made by the high school from BBC NEWS online, it can be considered that the school took it for granted that people shared same understanding of characteristic of school uniform; something that the students must wear in proper way without an exception [Force]. In addition, it seems that the point school tried to emphasize was that they had given enough consideration [Force] toward the characteristics and circumstances of the religion when they made school uniform. Therefore, there is no chance for Shabina Begum to get justice to wear jilbab but not the uniform. When Miss Begum's case was taking place in High Court, her representative, Yvonne Spencer, according to BBC NEWS online, last updated 27th May 2004, said that Miss Begum's chance of passing the important examination was taken away by being excluded from the school just because she did not wear school uniform [Force]. Although High Court did not give ruling that favors Miss Begum, it is understandable that Yvonne Spencer to emphasized Shabina Begum's right to take part in education as a method to fight back and win a suit. However when she won the case under the ruling of Court of Appeal, she made several statements which was mainly about her right to manifest religion and she did not mention about her right to have education particularly [Focus].

It seems to be appropriate that the school was fighting on the ground of school uniform, its characteristic and fairness of when making it. It is also to the point that Yvonne Spencer was fighting back on the ground of education to win the case. However, Shabina Begum was not on the same ground as the school was. She was not even on the same field [Force] as her representative. She did not make statements which were strongly connected [Force] to her right to have education as Yvonne Spencer was claiming on the trial or 
characteristic of school uniform and fairness of her school's uniform as her school was emphasizing. Her claim, as reading the statements she made that are written before, is more to do with the religion. Her claim was not just about school uniform versus jilbab, or whether jilbab should be accepted as school uniform. It was about how to live her life as good Muslim. Wearing jilbab is the way to show the God that she is living her life as good Muslim.

She had made several statements of how she feels about wearing jilbab and about the way of how she thinks of jilbab itself. The followings are the statements. "I feel it's an obligation on me to wear the jilbab as soon as I step outside my front door." (Begum 22 March 2006) "The jilbab is the clothing I feel was sent by the prophet." (Begum 22 March 2006) By reading these statements, it is quite obvious [Force] that she has very strong feeling [Force] toward wearing jilbab. As she states that to wear jilbab is "an obligation", wearing jilbab plays vital role in her daily life [Force] and also her mind, too. Therefore it cannot be separated from her life itself. In addition, it cannot be discussed only by the field [Force] of how characteristic of school uniform or how far a student can reflect her own wishes to the school uniform or education.

If one lives in secular society where religion does not play vital role in one's life, Shabina Begum's court case may sound extraordinary as she stepped out from her school and decided not to attend her lessons for more than a year for religious reason. Yet, she is not the only one who chose to wear veil in order to express what she believed in. The following is another example of women who chose to wear veil as method to express what she believed in.

Fareena Alam is a young British woman who has professional job, has received good education and was born in London but grew up in Singapore. She says in her article, "Beyond The Veil" written in Newsweek, unlike herself, none of her female family members chose to wear hijab, a headscarf. The sentences below are the reasons why she came to wear hijab.

"Driven by a strong sense of social justice and wanting to reconnect with my spirituality, I "found" Islam at university, where I was a campus activist. My decision to 
wear the head scarf, the hijab, at first had more to do with defining identity and a brash confidence about who I was and what values guided me. In time, it came to express my devotion as well." (Alam 30) As reading these sentences, it can be said that in the case of Fareena Alam, it was not her Islamic belief that made her wear veil at first, but she has something to share with Shabina Begum that they both decided to wear veil out of their intention to express what they believed in.

However, there is no absolute reason [Force] of why the women choose to wear veil that applies to every one of Muslim women. For those with ability of literacy, Qur'an and other reliable sources such as hadith -record of the Prophet's saying and doing- can be the reasons to wear veil. The interpretation of what the God tries to teach human being through Qur'an - written as a formation of poem - and other reliable sources is the responsibility of each individual, because there are no such people as clergyman but scholars in Islam, the scholars endeavor and interpret the Qur'an and other sources through their school of thoughts and there are several numbers of them [Force]. People seeks advice from scholars but since they have no clergyman or Pope- the head of all the Christians- who decides what is a heretic thought, in Islam, strictly speaking [Focus], there is no one to decide what is a heretic thought or true belief [Focus: sharp]. It is a responsibility of each individual to decide what to believe and not to believe. Therefore, it is each woman's responsibility and right to decide whether to wear veil or not.

As for Shabina Begum, she chose to wear veil out of her faith. However, interpretation of Qur'an and other sources are not only and the greatest reasons to wear veil. According to interview with professor lyama, whether they live in Muslim country or not, it is the Muslim society that makes or expect women to wear veil.

Fareena Alam says in her article about young British Muslims, herself included who are living outside of Muslim countries, that they are "the most globalized generation in European history connected to the countries of our parents or grandparents as well as to the broader spiritual community, umma, of world Islam." (Alam 32) According to her saying, young British Muslims are members of globalized society and also Muslim society. In addition, Muslim society is gaining its power according to Muslim journalist. He points 
out that for the Muslim in Britain today, the religion, Islam is becoming more influencing in daily lives and one's decision-making. (BBC NEWS 22 March 2006) As for Shabina Begum's court case, The Muslim Council of Britain (MCB), the UK's large Muslim community that profess themselves to be "UK's representative Muslim umbrella body" (MCB) made several announcement towards her court case through MCB press. They said that they see the decision which was made by High Court on 15 June 2004, as "worrying and objectionable." (MCB 15 June 2004) They also state that "Within this broad spectrum those that believe and choose to wear the jilbab and consider it to be part of their faith requirement of modest attire should be respected" (MCB 15 June 2004) However, they distinguish themselves from being a community to teach the interpretation and understanding of faith and this practice. As for Shabina Begum's victory under the ruling of House of Appeal, the head of MCB, Iqubal Sacranie made declaration as following. "This is a very important ruling on the issue of personal freedom." (BBC NEWS 02 March 2005)

The representative of British Muslim community did not make pronouncement that recommends women to wear veil as they state themselves to be "diverse community in terms of the interpretation and understanding of faith and its practice" (MCB 02 March 2005), but they did not show opposition toward Shabina Begum's action of going to court in order to get her right to wear jilbab. In fact, the Council considered her understanding of faith and its practice as respected.

Therefore, One consider under the circumstance as Islam greatly influences Muslim's daily life [Force] and decision-making that the fact of MCB making statement on Shabina Begum's court case and seeing her belief as respected proofs that Muslim community is indirectly expecting [Focus] and welcoming women to wear veil so that they can express that they are living their life as good Muslim. It also causes social impact on both Muslim community within Britain and that of none-Muslim's since they are affiliating 400 Muslim connected organizations and describes themselves as representative of British Muslim communities. MCB made Shabina Begum's court case a matter of their own rather than that of Miss Begum's by making those statements, and produced the environment where these kinds of acts will be respected. 
Whether Shabina Begum's action was for her, out of social presser that Muslim society has or purely out of her will to please God [Focus], one considers that the interpretation of faith and its practice of her should not be insulted since she considers that religious belief plays vital role in creation of her identity [Force] and cannot be separated from her life. As for her case, it can not be solved as seeing the issue only on the ground [Force] of how far a pupil can reflect her wishes to school uniform. It is deeper than discussion of school uniform. It is strongly related to creation of her identity [Force] and her life itself.

However, Muslim side should be aware that their community co-exists with that of British's. In the same way that Muslim society has its own culture, that British society has its own culture, too. As for British society, it needs to understand how wearing veil or any other religious activities have deep connection with Muslim's life.

There seem to be no effective solutions yet to be found within Europe. However, as beginning of finding one of them, let it start with filling up the gaps between two cultures caused by misinterpretation of heart of the issue. It is time for both Muslim society and British society to stop seeing the issue only through their perspectives [Force]. It will take $a$ huge amount of time [Force] and it may causes more complicated discussions to see things through different perspectives as religion seems to be at the core of Muslim's lives and as Europeans consider secularism is the greatest progress for them. However, as Fareena Alam described young British Muslims as "the most globalized generation within European history" in her article, it can be considered that there is a chance that the changes are going to be made. Again, considering what is written above, it is not straightforward problem that can be solved in one flick. However, it is the reality that there are Muslim societies within European societies. They both need to find the way to respect each other's cultures eventually and build a new society that is constructed of mixture of what the Europe had as their own culture and that of Muslim's.

\section{Case 2: Ai, complete essay marked for ATTITUDE}

In which language do deaf students study better, sign language or Japanese? The answer to this question has changed as the education at schools for deaf and mute 
students progressed. In the $19^{\text {th }}$ Century, the main method of education for the deaf was the aural method which forced students to practice understanding and speaking Japanese with a hearing aid. At that time, sign language was thought to be an obstacle which deterred students from acquiring Japanese skills. However, this idea started to fade away in Japan from the 1980s as bilingual education, which allows students to learn sign language first and then learn the language of the majority population, has become increasingly popular like in the United States. In the article "New School to Use Signs as 1st Language" (the daily Yomiuri on June 21, 2007), Keiko Katayama reports that the nation's first accredited school for students with auditory difficulties is planned to be founded in Tokyo next spring. Thus, more schools where deaf students can learn in sign language should be founded in Japan because of mainly four reasons; it would enable them to enter the academic world, offer them an opportunity to feel proud of being deaf and treated more equally in education. Besides, it would give deaf students the chance to have jobs in the future, and eventually lead to an improvement in public welfare for the hearing challenged as understanding grows in society.

. Sign Language Enables the Deaf to Acquire Academic Abilities

First, deaf students could learn what they are interested in as hearing students can with less difficulties and frustration if they could use sign language while studying. In the article "The Debate Over Deaf Education", Burton Bollag explains how important sign language is for the deaf to learn by introducing the example of a deaf person who has succeeded in academics. The student's name is Daniel S. Koo and he is deaf by nature. He started to go to a public school where he was required to speak and listen to Japanese with a hearing aid, which made him frustrated because he could hardly understand what his teacher were saying. In the result, he felt behind other hearing students academically. However, after he moved to a school where he could use hand movements, that is, sign, his academic success story started. He entered the University of Maryland at College Park keeping up with his classes with the help of an interpret and he later went on to Gallaudet University for graduate studies, where all the classes he took were conducted in American Sign Language. Finally, today he is at Georgetown University Medical Center doing a postdoc in neurolinguistics. In addition, using sign 
language allows deaf students to feel free to talk in class. According to the book "Tyokakushogeisha no nihongoshido niokeru shuwa no shiyo ni kansuru kenkyu (The research about use of sign language in teaching Japanese to people with hearing difficulties)" Chonan Hirohito, a professor at Tsukuba Technology University, states that deaf students use more adjective and adverbs when they use sign language than in cases of written or spoken Japanese based on his experiment concerning 36 deaf high school students. This means that they feel less reluctance [Judgment: positive] to speak in sign language and can express more details and richer content [Judgment: positive] and thus learn higher-level content in sign language [Judgment: positive]. From this research it can be said that sign language is indispensable as a deaf person's mother tongue [Appreciation: positive] and if it were not for sign language, the deaf would not have any language that they could use freely. For these reasons, deaf students should learn in sign language from elementary school so that they are ready to study academic subjects later.

. Sign Language Gives Deaf Students Confidence

The second strong point of using sign language at schools is that deaf students can feel proud of being deaf [Affect: positive]. Through the acquisition of sign language, the language they can use at their command, they would form their identities as a deaf individual. In the previously mentioned article "The Debate over Deaf Education," Burton cites a comment made by Mr. Koo, a deaf student who succeeded in academics. Mr. Koo explains that "ASL (American Sign Language) exposes children to the world's knowledge and it incorporates self-esteem and aspects of deaf culture." Deaf children can learn about what the deaf community is [Judgment: positive] or what it means to be deaf through sign language. In addition, in the book "Nihon shuwa to robunka (Japanese sign language and the deaf culture)," Kimura Harumi states that deaf students who go to Ryunoko Schooli, which proceeds bilingual and bicultural education ${ }^{\text {ii }}$ for deaf in Japanese sign language, have confidence that they can sign and are able to state their opinions and listen to others' opinions whether their parents are deaf or not.(Kimura, 264) Deaf students become able to communicate with others without feeling fear [Affect: positive] by using sign language, even with people who are not hearing impaired if there is an interpreter. Thus, acquisition of sign language is 
considerably important for deaf persons [Judgment: positive] to find their identities and self-esteem.

. Chance to have jobs

Thirdly, deaf students can have more chances to secure an occupation [Judgment: positive] in the future by making the most of there ability of signing improved in their school days. According to the resolution of the 11th World Congress of the World Federation of the Deafiii held in Tokyo, if students can learn each subject in sign language, they improve their abilities in various subjects, which eventually gives them more opportunities to select their job. Resulting from the spread of such ideas in Japan, an increasing number of deaf people have occupations and play a great part in making the society better. For instance, Ippuku, who is a deaf, entertains people as a Rakugokaiv according to the article by DINF v"Traditional Japanese Comedy Performed in Sign Language." Sign Language Rakugo came to draw much attention since the 1980s, a time when bilingual education for deaf and mute students became popular as mentioned in the Introduction. Thus, the experiences of learning sign language itself and other subjects in sign language are significant [Judgment: positive] for the deaf to increase their job opportunities in their future.

. Public facilities will be improved

Besides, public facilities will be improved as knowledge about deaf culture become more accessible as a result of the increasing number of deaf people who are active in the society. In the book "Chokaku gengo shogaisha to communication (People with linguistic and hearing difficulties and communication)," Ichibangase Yasuko states that for the improvement of the environment for the deaf it is necessary that people who are not hearing impaired understand about deaf culture and sign language to reduce barriers between them and deaf people. For example, New York has already began to improve public facilities for the deaf by approving agreements between consumer and state officials to offer 38 theaters with subtitles or narration in addition to the dozen theaters that now have such system(USA Today). Also, in Japan, Yahata General Hospital started to employ a sign language interpreter once a week on October.3rd (Sign Language Interpreter Was 
Placed). Thus, Japan should promote more familiarity between deaf people and others in society by drawing the attention of those who are not hearing impaired to the deaf community which would have the affect of increasing the number of schools for the deaf.

\section{. Lack of teachers}

It is true that a large number of teachers is needed to establish many schools for deaf students. The fact that there are two kinds of sign language in Japan makes the problem even more difficult [Appreciation: negative]. Japanese sign language is what deaf people usually use and Sign Japanese was created by a person with no hearing challenges for Japanese and actually for deaf children, who do not understand Japanese grammar, the latter is difficult to comprehend [Appreciation: negative]. Therefore, teachers who can sign using Japanese sign language are needed though only deaf persons can sign using it. However, if the number of schools for the deaf and programs for training deaf teachers increases, those who want to teach using their ability of signing could can make the situation better. This would give deaf people opportunities to have more jobs as teachers.

. Acquisition of Japanese

Moreover, some people might say that if deaf children use sign language at school, they cannot acquire Japanese language skills though they are Japanese. According to the article"Kowa kyoiku wa nihongo kyoiku"(Aural teaching is Japanese teaching)by an Internet school for the deaf, deaf children who learn sign language early in their life cannot acquire Japanese easily because for them Japanese is a foreign language. However, they do not have to practice using Japanese if they have already had their own language as Japanese do not have to learn another foreign language like English. In addition, through bilingual education they can learn written Japanese and have almost the same ability in writing and reading Japanese as people who are not hearing impaired.

\section{. Conclusion}

Thus, Japan should have more schools for deaf students where they can learn sign language because they could catch up to regular students [Judgment: positive] and learn 
academic subjects if they can use sign language while studying. Additionally, they would have more of a reason to feel proud [Affect: positive] of being deaf if they could communicate with others using sign language and the opportunities for deaf people to have a job would increase because they can find and improve their ability trying what they are interested in. Moreover, if there are a lot of deaf people who live actively in the society, public welfare would be made more convenient for deaf people. To increase the number of schools for deaf students from now on, more and more opportunities for people to know about the deaf community and the importance of sign language for deaf people.

\section{$<$ End Notes $>$}

${ }^{i}$ Ryunoko School was founded by NPO in Shinagawa Ward, Tokyo.

ii This is the slogan of Ryunoko School. Bilingual means Japanese sign language and written Japanes and bicultural means the deaf culture and the hearing culture.

iii The World Federation of the Deaf (WFD) is an international, non-governmental, central organization, whose members are from 123 countries.

iv Comic storytellers in Japan

v Disability INFormation Resources

\section{Case 2: Ai, complete essay marked for ENGAGEMENT}

In which language do deaf students study better, sign language or Japanese? The answer to this question has changed as the education at schools for deaf and mute students progressed. In the $19^{\text {th }}$ Century, the main method of education for the deaf was the aural method which forced students to practice understanding and speaking Japanese with a hearing aid. At that time, sign language was thought to be an obstacle [Attribution: hearsay] which deterred students from acquiring Japanese skills. However, this idea started to fade away in Japan from the 1980s as bilingual education, which allows students to learn sign language first and then learn the language of the majority population, has become increasingly popular like in the United States. In the article "New School to Use Signs as 1st Language" (the daily Yomiuri on June 21, 2007), Keiko Katayama reports [Attribution] that the nation's first accredited school for students with auditory difficulties is planned to be founded in Tokyo next spring. Thus, more schools where deaf students can learn in sign language should be founded [Modality: obligation] in Japan 
because of mainly four reasons; it would enable them [Expectation] to enter the academic world, offer them an opportunity [Expectation] to feel proud of being deaf and treated more equally in education. Besides, it would give deaf students the chance [Expectation] to have jobs in the future, and eventually lead to an improvement [Expectation] in public welfare for the hearing challenged as understanding grows in society.

. Sign Language Enables the Deaf to Acquire Academic Abilities

First, deaf students could learn what they are interested in [Expectation] as hearing students can with less difficulties and frustration if they could use sign language while studying. In the article "The Debate Over Deaf Education", Burton Bollag explains [Attribution] how important sign language is for the deaf to learn by introducing the example of a deaf person who has succeeded in academics. The student's name is Daniel S. Koo and he is deaf by nature. He started to go to a public school where he was required to speak and listen to Japanese with a hearing aid, which made him frustrated because he could hardly understand what his teacher were saying. In the result, he felt behind other hearing students academically. However, after he moved to a school where he could use hand movements, that is, sign, his academic success story started. He entered the University of Maryland at College Park keeping up with his classes with the help of an interpret and he later went on to Gallaudet University for graduate studies, where all the classes he took were conducted in American Sign Language. Finally, today he is at Georgetown University Medical Center doing a postdoc in neurolinguistics. In addition, using sign language allows deaf students to feel free to talk in class. According to the book "Tyokakushogeisha no nihongoshido niokeru shuwa no shiyo ni kansuru kenkyu (The research about use of sign language in teaching Japanese to people with hearing difficulties)" Chonan Hirohito, a professor at Tsukuba Technology University, states [Attribution] that deaf students use more adjective and adverbs when they use sign language than in cases of written or spoken Japanese based on his experiment concerning 36 deaf high school students. This means that they feel less reluctance to speak in sign language and can express more details and richer content and thus learn 
higher-level content in sign language. From this research it can be said that sign language is indispensable [Proclamation] as a deaf person's mother tongue and if it were not for sign language, the deaf would not have any language that they could use freely [Expectation]. For these reasons, deaf students should learn in sign language [Modality: obligation] from elementary school so that they are ready to study academic subjects later.

. Sign Language Gives Deaf Students Confidence

The second strong point of using sign language at schools is that deaf students can feel proud of being deaf. Through the acquisition of sign language, the language they can use at their command, they would form their identities as a deaf individual [Expectation]. In the previously mentioned article "The Debate over Deaf Education," Burton cites a comment made by Mr. Koo [Attribution], a deaf student who succeeded in academics. Mr. Koo explains [Attribution] that "ASL (American Sign Language) exposes children to the world's knowledge and it incorporates self-esteem and aspects of deaf culture." Deaf children can learn about what the deaf community is or what it means to be deaf through sign language. In addition, in the book "Nihon shuwa to robunka (Japanese sign language and the deaf culture)," Kimura Harumi states [Attribution] that deaf students who go to Ryunoko Schooli, which proceeds bilingual and bicultural education $^{\text {ii }}$ for deaf in Japanese sign language, have confidence that they can sign and are able to state their opinions and listen to others' opinions whether their parents are deaf or not.(Kimura, 264) Deaf students become able to communicate with others without feeling fear by using sign language, even with people who are not hearing impaired if there is an interpreter. Thus, acquisition of sign language is considerably important for deaf persons to find their identities and self-esteem.

\section{. Chance to have jobs}

Thirdly, deaf students can have more chances to secure an occupation in the future by making the most of there ability of signing improved in their school days. According to the resolution of the 11th World Congress [Attribution] of the World Federation of the Deafiii held in Tokyo, if students can learn each subject in sign language, they improve their 
abilities in various subjects, which eventually gives them more opportunities to select their job. Resulting from the spread of such ideas in Japan, an increasing number of deaf people have occupations and play a great part in making the society better. For instance, Ippuku, who is a deaf, entertains people as a Rakugoka ${ }^{\text {iv }}$ according to the article by DINF [Attribution] v"Traditional Japanese Comedy Performed in Sign Language." Sign Language Rakugo came to draw much attention since the 1980 s, a time when bilingual education for deaf and mute students became popular as mentioned in the Introduction. Thus, the experiences of learning sign language itself and other subjects in sign language are significant for the deaf to increase their job opportunities in their future.

. Public facilities will be improved

Besides, public facilities will be improved [Expectation] as knowledge about deaf culture become more accessible as a result of the increasing number of deaf people who are active in the society. In the book "Chokaku gengo shogaisha to communication (People with linguistic and hearing difficulties and communication)," Ichibangase Yasuko states [Attribution] that for the improvement of the environment for the deaf it is necessary that people who are not hearing impaired understand about deaf culture [Proclamation] and sign language to reduce barriers between them and deaf people. For example, New York has already began to improve public facilities for the deaf by approving agreements between consumer and state officials to offer 38 theaters with subtitles or narration in addition to the dozen theaters that now have such system(USA Today). Also, in Japan, Yahata General Hospital started to employ a sign language interpreter once a week on October.3rd (Sign Language Interpreter Was Placed). Thus, Japan should promote more familiarity [Modality: obligation] between deaf people and others in society by drawing the attention of those who are not hearing impaired to the deaf community which would have the affect of increasing the number of schools for the deaf [Expectation].

. Lack of teachers 
It is true that [Proclamation] a large number of teachers is needed to establish many schools for deaf students. The fact that there are two kinds [Proclamation] of sign language in Japan makes the problem even more difficult. Japanese sign language is what deaf people usually use and Sign Japanese was created by a person with no hearing challenges for Japanese and actually for deaf children, who do not understand Japanese grammar, the latter is difficult to comprehend. Therefore, teachers who can sign using Japanese sign language are needed though only deaf persons can sign using it. However, if the number of schools for the deaf and programs for training deaf teachers increases, those who want to teach using their ability of signing could can make the situation better [Modality: probability]. This would give deaf people opportunities to have more jobs as teachers [Expectation].

. Acquisition of Japanese

Moreover, some people might say that [Modality: probability/Attribution: hearsay] if deaf children use sign language at school, they cannot acquire Japanese language skills though they are Japanese. According to the article [Attribution]"Kowa kyoiku wa nihongo kyoiku"(Aural teaching is Japanese teaching)by an Internet school for the deaf, deaf children who learn sign language early in their life cannot acquire Japanese easily because for them Japanese is a foreign language. However, they do not have to practice using Japanese if they have already had their own language as Japanese do not have to learn another foreign language like English. In addition, through bilingual education they can learn written Japanese and have almost the same ability in writing and reading Japanese as people who are not hearing impaired.

\section{. Conclusion}

Thus, Japan should have more schools for deaf students [Modality: obligation] where they can learn sign language because they could catch up to regular students and learn academic subjects [Expectation] if they can use sign language while studying. Additionally, they would have more of a reason to feel proud of being deaf 
[Expectation] if they could communicate with others using sign language and the opportunities for deaf people to have a job would increase [Expectation] because they can find and improve their ability trying what they are interested in. Moreover, if there are a lot of deaf people who live actively in the society, public welfare would be made more convenient for deaf people [Expectation]. To increase the number of schools for deaf students from now on, more and more opportunities for people to know about the deaf community and the importance of sign language for deaf people.

\section{$<$ End Notes $>$}

${ }^{i}$ Ryunoko School was founded by NPO in Shinagawa Ward, Tokyo.

ii This is the slogan of Ryunoko School. Bilingual means Japanese sign language and written Japanes and bicultural means the deaf culture and the hearing culture.

iii The World Federation of the Deaf (WFD) is an international, non-governmental, central organization, whose members are from 123 countries.

iv Comic storytellers in Japan

v Disability INFormation Resources

\section{Case 2: Ai, complete essay marked for GRADUATION}

In which language do deaf students study better, sign language or Japanese? The answer to this question has changed as the education at schools for deaf and mute students progressed. In the $19^{\text {th }}$ Century, the main method of education for the deaf was the aural method which forced students to practice [Force] understanding and speaking Japanese with a hearing aid. At that time, sign language was thought to be an obstacle which deterred students from acquiring Japanese skills. However, this idea started to fade away in Japan from the 1980s as bilingual education, which allows students to learn sign language first and then learn the language of the majority population, has become increasingly popular [Force] like in the United States. In the article "New School to Use Signs as 1st Language" (the daily Yomiuri on June 21, 2007), Keiko Katayama reports that the nation's first accredited school for students with auditory difficulties is planned to be founded in Tokyo next spring. Thus, more schools where deaf students can learn in sign language should be founded in Japan because of mainly four reasons; it would enable them to enter the academic world, offer them an opportunity to feel proud of being deaf and 
treated more equally in education. Besides, it would give deaf students the chance to have jobs in the future, and eventually lead to an improvement in public welfare for the hearing challenged as understanding grows in society.

. Sign Language Enables the Deaf to Acquire Academic Abilities

First, deaf students could learn what they are interested in as hearing students can with less difficulties and frustration if they could use sign language while studying. In the article "The Debate Over Deaf Education", Burton Bollag explains how important sign language is for the deaf to learn by introducing the example of a deaf person who has succeeded in academics. The student's name is Daniel S. Koo and he is deaf by nature. He started to go to a public school where he was required to speak and listen to Japanese with a hearing aid, which made him frustrated because he could hardly understand [Force] what his teacher were saying. In the result, he felt behind other hearing students academically. However, after he moved to a school where he could use hand movements, that is, sign [Force: synonymy], his academic success story started. He entered the University of Maryland at College Park keeping up with his classes with the help of an interpret and he later went on to Gallaudet University for graduate studies, where all the classes he took were conducted in American Sign Language. Finally, today he is at Georgetown University Medical Center doing a postdoc in neurolinguistics. In addition, using sign language allows deaf students to feel free to talk in class. According to the book "Tyokakushogeisha no nihongoshido niokeru shuwa no shiyo ni kansuru kenkyu (The research about use of sign language in teaching Japanese to people with hearing difficulties)" Chonan Hirohito, a professor at Tsukuba Technology University, states that deaf students use more adjective and adverbs when they use sign language than in cases of written or spoken Japanese based on his experiment concerning 36 deaf high school students. This means that they feel less reluctance to speak in sign language and can express more details and richer content and thus learn higher-level content in sign language. From this research it can be said that sign language is indispensable as a deaf person's mother tongue and if it were not for sign language, the deaf would not have any language that they could use freely. For these reasons, deaf students should 
learn in sign language from elementary school so that they are ready to study academic subjects later.

. Sign Language Gives Deaf Students Confidence

The second strong point of using sign language [Force] at schools is that deaf students can feel proud of being deaf. Through the acquisition of sign language, the language they can use at their command, they would form their identities as a deaf individual. In the previously mentioned article "The Debate over Deaf Education," Burton cites a comment made by Mr. Koo, a deaf student who succeeded in academics. Mr. Koo explains that "ASL (American Sign Language) exposes children to the world's knowledge and it incorporates self-esteem and aspects of deaf culture." Deaf children can learn about what the deaf community is or what it means to be deaf through sign language. In addition, in the book "Nihon shuwa to robunka (Japanese sign language and the deaf culture)," Kimura Harumi states that deaf students who go to Ryunoko Schooli, which proceeds bilingual and bicultural education ${ }^{\text {ii }}$ for deaf in Japanese sign language, have confidence that they can sign and are able to state their opinions and listen to others' opinions whether their parents are deaf or not.(Kimura, 264) Deaf students become able to communicate with others without feeling fear by using sign language, even with people who are not hearing impaired [Force: intensifier] if there is an interpreter. Thus, acquisition of sign language is considerably important [Force: intensifier] for deaf persons to find their identities and self-esteem.

. Chance to have jobs

Thirdly, deaf students can have more chances to secure an occupation in the future by making the most of there ability of signing improved in their school days. According to the resolution of the 11th World Congress of the World Federation of the Deafii held in Tokyo, if students can learn each subject in sign language, they improve their abilities in various subjects, which eventually gives them more opportunities to select their job. Resulting from the spread of such ideas in Japan, an increasing number of deaf people have occupations and play a great part in making the society better [Force: intensifier]. For instance, Ippuku, who is a deaf, entertains people as a Rakugokaiv according to the article 
by DINF v"Traditional Japanese Comedy Performed in Sign Language." Sign Language Rakugo came to draw much attention since the 1980s, a time when bilingual education for deaf and mute students became popular as mentioned in the Introduction. Thus, the experiences of learning sign language itself and other subjects in sign language are significant for the deaf to increase their job opportunities in their future.

. Public facilities will be improved

Besides, public facilities will be improved as knowledge about deaf culture become more accessible as a result of the increasing number of deaf people who are active in the society. In the book "Chokaku gengo shogaisha to communication (People with linguistic and hearing difficulties and communication)," Ichibangase Yasuko states that for the improvement of the environment for the deaf it is necessary that people who are not hearing impaired understand about deaf culture and sign language to reduce barriers between them and deaf people. For example, New York has already began to improve public facilities for the deaf by approving agreements between consumer and state officials to offer 38 theaters with subtitles or narration in addition to the dozen theaters that now have such system(USA Today). Also, in Japan, Yahata General Hospital started to employ a sign language interpreter once a week on October.3rd (Sign Language Interpreter Was Placed). Thus, Japan should promote more familiarity between deaf people and others in society by drawing the attention of those who are not hearing impaired to the deaf community which would have the affect of increasing the number of schools for the deaf.

\section{. Lack of teachers}

It is true that a large number of teachers is needed to establish many schools for deaf students. The fact that there are two kinds of sign language in Japan makes the problem even more difficult [Force: intensifier]. Japanese sign language is what deaf people usually use and Sign Japanese was created by a person with no hearing challenges for Japanese and actually for deaf children, who do not understand Japanese grammar, the latter is difficult to comprehend. Therefore, teachers who can sign using Japanese sign language are needed though only deaf persons can sign using it. However, if the number of schools for the deaf and programs for training deaf teachers increases, those who want to teach using their 
ability of signing could can make the situation better. This would give deaf people opportunities to have more jobs as teachers.

\section{. Acquisition of Japanese}

Moreover, some people might say that if deaf children use sign language at school, they cannot acquire Japanese language skills though they are Japanese. According to the article"Kowa kyoiku wa nihongo kyoiku"(Aural teaching is Japanese teaching)by an Internet school for the deaf, deaf children who learn sign language early in their life cannot acquire Japanese easily because for them Japanese is a foreign language. However, they do not have to practice using Japanese if they have already had their own language as Japanese do not have to learn another foreign language like English. In addition, through bilingual education they can learn written Japanese and have almost the same ability [Force] in writing and reading Japanese as people who are not hearing impaired.

\section{. Conclusion}

Thus, Japan should have more schools for deaf students where they can learn sign language because they could catch up to regular students and learn academic subjects if they can use sign language while studying. Additionally, they would have more of a reason to feel proud of being deaf if they could communicate with others using sign language and the opportunities for deaf people to have a job would increase because they can find and improve their ability trying what they are interested in. Moreover, if there are a lot of deaf people who live actively in the society, public welfare would be made more convenient for deaf people. To increase the number of schools for deaf students from now on, more and more opportunities [Force: repetition] for people to know about the deaf community and the importance of sign language for deaf people.

$<$ End Notes $>$

i Ryunoko School was founded by NPO in Shinagawa Ward, Tokyo.

ii This is the slogan of Ryunoko School. Bilingual means Japanese sign language and written Japanes and bicultural means the deaf culture and the hearing culture. 
iii The World Federation of the Deaf (WFD) is an international, non-governmental, central organization, whose members are from 123 countries.

iv Comic storytellers in Japan

v Disability INFormation Resources

\section{Case 3: Rika, complete essay marked for ATTITUDE}

Tens of millions of animals are killed by animal testing every year (Feder). Animals have been used for disease research from long ago, and many still think animal testing is the best way to check if medicine is safe or not. This is because people only think about their own profits [Judgment: negative] and do not think about animals' rights. Today, the number of people who are against animal testing is increasing and they say that there is no need for animal testing anymore. Using animals for disease research should be stopped because it is not the most reliable method [Appreciation: negative] for treating human health problems, too much tax money is used for the experiments [Appreciation: negative] and it is inhumane to use animals just for the profit of humans [Judgment: negative].

To start with, animal testing is not the most reliable method [Appreciation: negative] for treating human health problems. Many types of medicine proven to be safe by animal testing had side effects on humans. Even though tested medicine may be safe on animals, it does not always mean it will be safe for humans too. There is no way to prove that the medicine will be safe for people if it is not checked on humans first. It is said that as much as twenty five percent of the medicines which was checked by animal testing could not show side effects which subsequently proved serious enough to prevent those medicines to be sold (Feder). This means it is very dangerous to use animal testing [Appreciation: negative] to prove whether or not medicine will be safe because serious side effects may show on humans even though it was not shown on animals. Also, incorrect data from animal testing leads to incorrect knowledge for treatment [Appreciation: negative]. If people continue to rely on data from animals, there will be a risk of more people suffering from side effects [Appreciation: negative]. The number of people who want to receive treatment without using animal testing is increasing, so it will not be long before animal testing becomes obsolete. 
Secondly, too much tax money is used for animal experiments [Appreciation: negative]. Although a huge amount money was used to research cancer, nearly three million people were killed by malignant neoplasm (1), and the number of patients dying is still increasing for fifty years (ANIMAL RIGHTS CENTER). Animal testing costs a lot of money [Appreciation: negative]. Before medicine is tested on animals, animals have to be taken care of until that time. The money is used for food, maintaining the facilities for animals to live. If people knew how their tax money was being used for animal testing despite no reliable results from research, most people would condemn the use of their tax money for animal testing [Judgment: negative]. Instead, tax money currently used for animals testing should be used for environmental conservation and pollution preservation.

Money for environmental conservation and pollution preservation is more needed than animal testing. Akio Morishima head of the Institute of Global Environmental Studies at Sophia University "Without doubt, the most important problem in the next century, both for Japan and for the world, will be global warming." (Corliss). This means that environmental problems are becoming more and more serious [Appreciation: negative], so it is better to use money to prevent those problems rather than use money to take unreliable data [Appreciation: negative] from animal testings. Improvement of environmental circumstances and the standard of living is just as important as the development of medical care. For example, even if people are saved from dying as a result of medical care, their continued lives are not guranteed if the standard of living are poor. From this perspective, it is much more efficient to use money for improving people's standard of living rather than kill innocent animals in vain.

Lastly, it is inhumane to use animals just for the profit of humans [Judgment: negative]. It is possible for animals to be used for research because they cannot speak and are weaker than humans [Appreciation: negative]. Not being able to speak, however, does not mean they do not have feelings [Affect: negative]. Although animals cannot speak human language, it is not difficult to imagine that they will suffer when medicine does not work [Affect: negative]. Also, animals are forced to be ill [Judgment: negative] even though they are healthy, and some of them are born solely to be used for research. Animals born for the sole purpose of research could not possibly imagine that they were born to be 
killed [Affect: negative]. Fumie Hattori association president of JAVA said, "People don't know about the issues. Because they don't know they can be silent about them. When they do learn what's going on there's often a good response," (Bayer). Still, many people are only thinking about themselves [Judgment: negative] and not thinking about animals killed by animal testing. Animals have the right to live too, and animal testing is an infringement on their rights [Appreciation: negative].

Although there are many negative points about animal testing_[Appreciation: negative], there are still many people who say that animal testing should be continued. They argue that it would not be possible to make medicine to cure diseases if it was not for animal testing, and that most people who received the Nobel Prize for Medicine carried out such experiments (Pro-Test standing up for science). However, 'HUMAN skin, eyes, the lining of the throat - snippets of these and other tissues are now routinely grown in test tubes from donated human cells. The goal is not to patch up ailing people but to use the human tissues in place of mice, dogs or other lab animals for testing new drugs, cosmetics and other products." (Feder). There are alternatives to animal testings today, which do not cost as much as animal testing and are effective and humane [Appreciation: positive] (JAVA Doubutsu jikken haishi wo motomeru kai). In consideration of effective alternatives, there is no need to torture animals during disease research, and people can receive the Nobel Prize without sacrificing them. Improvement of technology allows alternatives to be used instead of animals [Appreciation: positive], so animals should be freed and people should think about how to live in the world peacefully together with animals.

In conclusion, improvement of technology enabled companies to test medicines without animal testing and use alternatives such as human skins instead [Appreciation: positive], and many people are becoming aware [Judgment: positive] that it is difficult to rely on animal testing to treat human health problems, the amount of tax money used for the experiment is too much [Appreciation: negative] and it is no thinking about animals rights, so it is more likely to say that there is no need for animal testing any more. If more people posses the knowledge of how many animals are killed and how they are treated during animal testing, more people would fight for the improvement of animals' rights. Also, if people can dismiss their preconceptions about medicine which passed animal testing as 
being safe, more people will take notice of the effective alternatives. It is not just humans who are living in the world [Judgment: negative]. It is time for humans to take action and fight for peace, both for humans themselves and for animals [Judgment: positive].

\section{Case 3: Rika, complete essay marked for ENGAGEMENT}

Tens of millions of animals are killed by animal testing every year (Feder). Animals have been used for disease research from long ago, and many still think animal testing is the best way [Attribution] to check if medicine is safe or not. This is because people only think about their own profits and do not think about animals' rights. Today, the number of people who are against animal testing is increasing and they say that there is no need for animal testing anymore [Attribution]. Using animals for disease research should be stopped [Modality: obligation] because it is not the most reliable method for treating human health problems, too much tax money is used for the experiments and it is inhumane to use animals just for the profit of humans.

To start with, animal testing is not the most reliable method for treating human health problems. Many types of medicine proven to be safe by animal testing had side effects on humans. Even though tested medicine may be safe on animals, it does not always mean it will be safe [Modality: probability] for humans too. There is no way to prove that the medicine will be safe [Proclamation] for people if it is not checked on humans first. It is said that as much as twenty five percent [Attribution] of the medicines which was checked by animal testing could not show side effects which subsequently proved serious enough to prevent those medicines to be sold (Feder). This means it is very dangerous to use animal testing to prove whether or not medicine will be safe because serious side effects may show on humans [Modality: probability] even though it was not shown on animals. Also, incorrect data from animal testing leads to incorrect knowledge for treatment. If people continue to rely on data from animals, there will be a risk of more people suffering from side effect [Expectation]. The number of people who want 
to receive treatment without using animal testing is increasing, so it will not be long before animal testing becomes obsolete [Expectation].

Secondly, too much tax money is used for animal experiments. Although a huge amount money was used to research cancer, nearly three million people were killed by malignant neoplasm (1), and the number of patients dying is still increasing for fifty years (ANIMAL RIGHTS CENTER). Animal testing costs a lot of money. Before medicine is tested on animals, animals have to be taken care of until that time. The money is used for food, maintaining the facilities for animals to live. If people knew how their tax money was being used for animal testing despite no reliable results from research, most people would condemn the use of their tax money for animal testing [Expectation]. Instead, tax money currently used for animals testing should be used for [Modality: obligation] environmental conservation and pollution preservation.

Money for environmental conservation and pollution preservation is more needed than animal testing. Akio Morishima head of the Institute of Global Environmental Studies at Sophia University [Attribution] "Without doubt, the most important problem in the next century, both for Japan and for the world, will be global warming." (Corliss). This means that environmental problems are becoming more and more serious, so it is better to use money to prevent those problems rather than use money to take unreliable data from animal testings. Improvement of environmental circumstances and the standard of living is just as important as the development of medical care. For example, even if people are saved from dying as a result of medical care, their continued lives are not guranteed if the standard of living are poor. From this perspective, it is much more efficient to use money for improving people's standard of living rather than kill innocent animals in vain.

Lastly, it is inhumane to use animals just for the profit of humans. It is possible for animals to be used for research because they cannot speak and are weaker than humans. Not being able to speak, however, does not mean they do not have feelings. Although animals cannot speak human language, it is not difficult to imagine that they will suffer [Expectation] when medicine does not work. Also, animals are forced to be ill even though they are 
healthy, and some of them are born solely to be used for research. Animals born for the sole purpose of research could not possibly imagine that they were born to be killed. Fumie Hattori association president of JAVA said [Attribution], "People don't know about the issues. Because they don't know they can be silent about them. When they do learn what's going on there's often a good response," (Bayer). Still, many people are only thinking about themselves and not thinking about animals killed by animal testing. Animals have the right to live too, and animal testing is an infringement on their rights.

Although there are many negative points about animal testing, there are still many people who say that animal testing should be continued [Attribution]. They argue that it would not be possible to make medicine to cure diseases [Attribution] if it was not for animal testing, and that most people who received the Nobel Prize for Medicine carried out such experiments (Pro-Test standing up for science). However, 'HUMAN skin, eyes, the lining of the throat - snippets of these and other tissues are now routinely grown in test tubes from donated human cells. The goal is not to patch up ailing people but to use the human tissues in place of mice, dogs or other lab animals for testing new drugs, cosmetics and other products." (Feder). There are alternatives to animal testings today, which do not cost as much as animal testing and are effective and humane (JAVA Doubutsu jikken haishi wo motomeru kai). In consideration of effective alternatives, there is no need to torture animals [Proclamation] during disease research, and people can receive the Nobel Prize without sacrificing them. Improvement of technology allows alternatives to be used instead of animals, so animals should be freed [Modality: obligation] and people should think about how to live in the world peacefully together with animals [Modality: obligation].

In conclusion, improvement of technology enabled companies to test medicines without animal testing and use alternatives such as human skins instead, and many people are becoming aware that it is difficult to rely on animal testing to treat human health problems, the amount of tax money used for the experiment is too much and it is no thinking about animals rights, so it is more likely to say [Modality: probability] that there is no need for animal testing any more [Proclamation]. If more people posses the knowledge of how many animals are killed and how they are treated during animal testing, 
more people would fight for the improvement of animals' right [Expectation]. Also, if people can dismiss their preconceptions about medicine [Modality: probability] which passed animal testing as being safe, more people will take notice of the effective alternatives [Expectation]. It is not just humans who are living in the world. It is time for humans to take action [Proclamation] and fight for peace, both for humans themselves and for animals.

\section{Case 3: Rika, complete essay marked for GRADUATION}

Tens of millions of animals are killed by animal testing every year (Feder). Animals have been used for disease research from long ago, and many [Force] still think animal testing is the best way to check if medicine is safe or not. This is because people only [Force: intensifier] think about their own profits and do not think about animals' rights. Today, the number of people who are against animal testing is increasing and they say that there is no need for animal testing anymore. Using animals for disease research should be stopped because it is not the most reliable method for treating human health problems, too much tax money [Force] is used for the experiments and it is inhumane to use animals just [Force: intensifier] for the profit of humans.

To start with, animal testing is not the most reliable method for treating human health problems. Many types [Force] of medicine proven to be safe by animal testing had side effects on humans. Even though tested medicine may be safe on animals, it does not always mean [Force: negative intensifier] it will be safe for humans too. There is no way to prove that the medicine will be safe for people if it is not checked on humans first. It is said that as much as twenty five percent of the medicines which was checked by animal testing could not show side effects which subsequently proved serious enough to prevent those medicines to be sold (Feder). This means it is very dangerous [Force: intensifier] to use animal testing to prove whether or not medicine will be safe because serious side effects [Force] may show on humans even though it was not shown on animals. Also, incorrect data from animal testing leads to incorrect knowledge for treatment. If people continue to rely on data from animals, there will be a risk of more people suffering from side effects. 
The number of people who want to receive treatment without using animal testing is increasing, so it will not be long before animal testing becomes obsolete.

Secondly, too much tax money [Force] is used for animal experiments. Although a huge amount money [Force] was used to research cancer, nearly three million people were killed by malignant neoplasm (1), and the number of patients dying is still increasing for fifty years (ANIMAL RIGHTS CENTER). Animal testing costs a lot of money [Force]. Before medicine is tested on animals, animals have to be taken care of until that time. The money is used for food, maintaining the facilities for animals to live. If people knew how their tax money was being used for animal testing despite no reliable results from research, most people would condemn the use of their tax money for animal testing. Instead, tax money currently used for animals testing should be used for environmental conservation and pollution preservation.

Money for environmental conservation and pollution preservation is more needed than animal testing. Akio Morishima head of the Institute of Global Environmental Studies at Sophia University "Without doubt, the most important problem in the next century, both for Japan and for the world, will be global warming." (Corliss). This means that environmental problems are becoming more and more serious [Force: repetition], so it is better to use money to prevent those problems rather than use money to take unreliable data from animal testings. Improvement of environmental circumstances and the standard of living is just as important [Force: intensifier] as the development of medical care. For example, even if people are saved [Force] from dying as a result of medical care, their continued lives are not guranteed if the standard of living are poor. From this perspective, it is much more efficient [Force] to use money for improving people's standard of living rather than kill innocent animals in vain [Force: negative].

Lastly, it is inhumane to use animals just for the profit of humans. It is possible for animals to be used for research because they cannot speak and are weaker than humans. Not being able to speak, however, does not mean they do not have feelings. Although animals cannot speak human language, it is not difficult to imagine that they will suffer when medicine does not work. Also, animals are forced to be ill even though they are healthy, and some of 
them are born solely to be used for research [Focus]. Animals born for the sole purpose of research could not possibly imagine that they were born to be killed. Fumie Hattori association president of JAVA said, "People don't know about the issues. Because they don't know they can be silent about them. When they do learn what's going on there's often a good response," (Bayer). Still, many people [Force: intensifier] are only thinking [Force: intensifier] about themselves and not thinking about animals killed by animal testing. Animals have the right to live too, and animal testing is an infringement on their rights.

Although there are many negative points [Force: intensifier] about animal testing, there are still many people [Force: intensifier] who say that animal testing should be continued. They argue that it would not be possible to make medicine to cure diseases if it was not for animal testing, and that most people who received the Nobel Prize for Medicine carried out such experiments (Pro-Test standing up for science). However, 'HUMAN skin, eyes, the lining of the throat - snippets of these and other tissues are now routinely grown in test tubes from donated human cells. The goal is not to patch up ailing people but to use the human tissues in place of mice, dogs or other lab animals for testing new drugs, cosmetics and other products." (Feder). There are alternatives to animal testings today, which do not cost as much as animal testing and are effective and humane (JAVA Doubutsu jikken haishi wo motomeru kai). In consideration of effective alternatives, there is no need to torture animals during disease research, and people can receive the Nobel Prize without sacrificing them. Improvement of technology allows alternatives to be used instead of animals, so animals should be freed and people should think about how to live in the world peacefully together with animals.

In conclusion, improvement of technology enabled companies to test medicines without animal testing and use alternatives such as human skins instead, and many people [Force: intensifier] are becoming aware that it is difficult to rely on animal testing to treat human health problems, the amount of tax money used for the experiment is too much and it is no thinking about animals rights, so it is more likely to say that there is no need for animal testing any more. If more people posses the knowledge of how many animals are killed and how they are treated during animal testing, more people would fight for the improvement of animals' rights. Also, if people can dismiss their preconceptions about medicine which 
passed animal testing as being safe, more people will take notice of the effective alternatives. It is not just humans [Force: intensifier] who are living in the world. It is time for humans to take action and fight for peace, both for humans themselves and for animals.

\section{Case 4: Yui, complete essay marked for ATTITUDE}

In recent years, global warming has become a worldwide issue. Ice and glaciers are melting, the world's average temperature is rising, and irregular weather is causing many people to suffer [Appreciation: negative] and plants and animals to become extinct. The world seems like it's coming to an end. Although these problems from global warming are happening, there are some things we can all do to make this situation better.

First of all, we should stop wasting energy. There are a many simple ways we can do this. First, always remember to turn off the lights when leaving the room [Judgment: positive]. Second, do not let the water run while brushing your teeth or washing your face [Judgment: negative]. Third, do not set the air conditioner to an excessive temperature [Judgment: negative]. All these points stated seems like a little thing, but every degree, every minute lights are turned off, every millimeter of water saved, will change the world [Appreciation: positive] when it is done by many people. Just always keep in mind the word "mottainai" and you'll be somewhat contributing towards saving energy [Judgment: positive].

The second thing we can do to prevent global warming is to have minimal trash. Just say you do not need a plastic bag when going grocery shopping and say you do not need chopsticks when buying food at the convenience store [Judgment: positive]. Carry your own shopping bag and your own chopsticks [Judgment: positive]. Another thing is that companies making candies and snacks should consider overpackaging [Appreciation: negative]. There is no need to wrap every candy, then wrap it again with a bigger bag, then put in a bag, then wrap the box, then put it in a box again [Judgment: negative]. Once is enough for wrapping [Appreciation: negative]. If people consider overpackaging [Judgment: positive], it will lead to us having less trash [Appreciation: positive]. 
Thirdly, to prevent global warming, we should rethink about our way of transporting. There is no need to use cars to go somewhere nearby [Judgment: negative]. Walk to the nearest station and take buses and trains [Judgment: positive]. Also, do not use one car per person [Judgment: negative]. We should start carpooling with friends [Judgment: positive]. This means fewer vehicles, which means less carbon dioxide in the air [Appreciation: positive].

As a conclusion, there are many solutions for what we can do to prevent global warming. There are things we can start from today to save the world. All of these solutions listed have to be done by many people, not just one person. We should all reconsider about what we have to do to prevent global warming, which is necessary to save our own lives.

\section{Case 4: Yui, complete essay marked for ENGAGEMENT}

In recent years, global warming has become a worldwide issue. Ice and glaciers are melting, the world's average temperature is rising, and irregular weather is causing many people to suffer and plants and animals to become extinct. The world seems like it's coming to an end [Reality phase]. Although these problems from global warming are happening, there are some things we can all do to make this situation better.

First of all, we should stop wasting energy [Modality: obligation]. There are a many simple ways we can do this. First, always remember to turn off the lights when leaving the room. Second, do not let the water run while brushing your teeth or washing your face. Third, do not set the air conditioner to an excessive temperature. All these points stated seems like a little thing [Reality phase], but every degree, every minute lights are turned off, every millimeter of water saved, will change the world when it is done by many people. Just always keep in mind the word "mottainai" and you'll be somewhat contributing towards saving energy.

The second thing we can do to prevent global warming is to have minimal trash [Modality: obligation]. Just say you do not need a plastic bag when going grocery shopping and say you do not need chopsticks when buying food at the convenience store. Carry your own shopping bag and your own chopsticks. Another thing is that companies making candies and snacks should consider overpackaging. There is no need to wrap every 
candy [Proclamation], then wrap it again with a bigger bag, then put in a bag, then wrap the box, then put it in a box again. Once is enough for wrapping. If people consider overpackaging, it will lead to us having less trash.

Thirdly, to prevent global warming, we should rethink about our way of transporting [Modality: obligation]. There is no need to use cars to go somewhere nearby [Proclamation]. Walk to the nearest station and take buses and trains. Also, do not use one car per person. We should start carpooling [Modality: obligation] with friends. This means fewer vehicles, which means less carbon dioxide in the air.

As a conclusion, there are many solutions for what we can do to prevent global warming. There are things we can start from today to save the world. All of these solutions listed have to be done by many people [Modality: obligation], not just one person. We should all reconsider about what we have to do [Modality: obligation] to prevent global warming, which is necessary to save our own lives [Modality: obligation].

\section{Case 4: Yui, complete essay marked for GRADUATION}

In recent years, global warming has become a worldwide issue. Ice and glaciers are melting, the world's average temperature is rising, and irregular weather is causing many people [Force] to suffer and plants and animals to become extinct. The world seems like it's coming to an end. Although these problems from global warming are happening, there are some things we can all do to make this situation better.

First of all, we should stop wasting energy. There are a many simple ways we can do this. First, always remember to turn off the lights when leaving the room. Second, do not let the water run while brushing your teeth or washing your face. Third, do not set the air conditioner to an excessive temperature. All these points stated seems like a little thing, but every degree, every minute lights are turned off, every millimeter of water saved [Force:

repetition], will change the world when it is done by many people [Force]. Just always keep in mind [Force: intensifier] the word "mottainai" and you'll be somewhat contributing [Force] towards saving energy. 
The second thing we can do to prevent global warming is to have minimal trash. Just say you [Force] do not need a plastic bag when going grocery shopping and say you do not need chopsticks when buying food at the convenience store. Carry your own shopping bag and your own chopsticks. Another thing is that companies making candies and snacks should consider overpackaging. There is no need to wrap every candy [Force], then wrap it again with a bigger bag, then put in a bag, then wrap the box, then put it in a box again [Force: repetition]. Once is enough for wrapping. If people consider overpackaging, it will lead to us having less trash.

Thirdly, to prevent global warming, we should rethink about our way of transporting. There is no need to use cars to go somewhere nearby. Walk to the nearest station and take buses and trains. Also, do not use one car per person. We should start carpooling with friends. This means fewer vehicles, which means less carbon dioxide in the air.

As a conclusion, there are many solutions [Force] for what we can do to prevent global warming. There are things we can start from today to save the world. All of these solutions listed have to be done by many people [Force], not just one person [Force]. We should all reconsider [Force] about what we have to do to prevent global warming, which is necessary to save our own lives. 